\title{
Gesellschaftliche Bewertung der Landnutzungsstrategien
}

\author{
Peter Elsasser, Ulrike Grabski-Kieron, Meike Hellmich, Jesko \\ Hirschfeld, Mathias Raabe, Sandra Rajmis, Julian Sagebiel, Rosemaire \\ Siebert, Annett Steinführer, Reimund Steinhäußer und Priska Weller
}

\section{Inhaltsverzeichnis}

5.1 Ökonomische Bewertung von Ökosystemleistungen der Landnutzung . . . . . . . . . 301

5.1.1 Überblick über Daten und Methoden zur ökonomischen Bewertung von

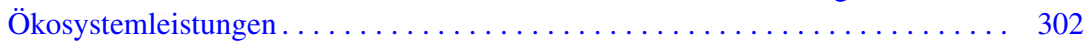

5.1.1.1 Bereitstellende Ökosystemleistungen.................. 303

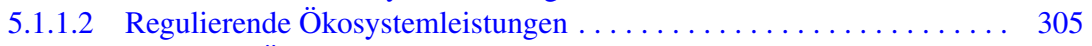

5.1.1.3 Kulturelle Ökosystemleistungen................... 306

5.1.2 Ergebnisse der ökonomischen Bewertung der Ökosystemleistungen

landwirtschaftlicher Landnutzungen . . . . . . . . . . . . . . . . . . . . . 309

5.1.2.1 Bereitstellende Ökosystemleistungen landwirtschaftlicher Landnutzungen . . . . . . . . . . . . . . . . . . . . . . . 309

5.1.2.2 Regulierende Ökosystemleistungen landwirtschaftlicher

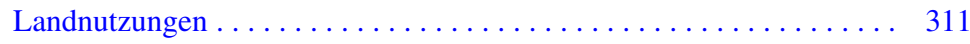

P. Elsasser $(\bowtie) \cdot$ P. Weller

Thünen-Institut für Internationale Waldwirtschaft und Forstökonomie,

Hamburg-Bergedorf, Deutschland

E-Mail: cc-landstrad@thuenen.de

U. Grabski-Kieron · M. Raabe

Westfälische Wilhelms-Universität Münster, Münster, Deutschland

M. Hellmich · A. Steinführer

Thünen-Institut für Ländliche Räume, Braunschweig, Deutschland

J. Hirschfeld $\cdot$ S. Rajmis $\cdot$ J. Sagebiel

Institut für ökologische Wirtschaftsforschung (IÖW), Berlin, Deutschland

R. Siebert $\cdot$ R. Steinhäußer

Leibniz-Zentrum für Agrarlandschaftsforschung (ZALF) e. V., Müncheberg, Deutschland

(C) Der/die Autor(en) 2021

H. Gömann und J. Fick (Hrsg.), Wechselwirkungen zwischen Landnutzung und

Klimawandel, https://doi.org/10.1007/978-3-658-18671-5_5 
5.1.2.3 Kulturelle Ökosystemleistungen landwirtschaftlicher Landnutzungen . 314

5.1.2.4 Aggregierte Ergebnisse der ökonomischen Bewertung der

Ökosystemleistungen der landwirtschaftlichen

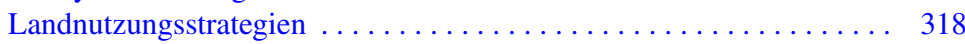

5.1.3 Ökonomische Bewertung der kulturellen Ökosystemleistungen des Waldes . . . 321

5.1.3.1 Ergebnisse zu Naturschutz- und Landschaftspflegeleistungen

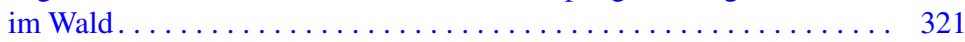

5.1.3.2 Einordnung der Ergebnisse und weiterer Leistungen des Waldes . . . . 328

5.1.3.3 Aggregierte Ergebnisse der ökonomischen Bewertung der

forstwirtschaftlichen Managementstrategien ............. 331

5.1.4 Diskussion und Ausblick zur ökonomischen Bewertung von

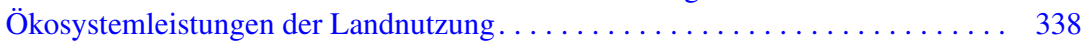

5.2 Institutionelle Gestaltungsoptionen für eine nachhaltige Landnutzung. . . . . . . . . 341

5.2.1 Einführung, Zielsetzung und methodische Vorgehensweise . . . . . . . . . . . 342

5.2.2 Ergebnisse der planungswissenschaftlichen Evaluierung . . . . . . . . . . . . . 345

5.2.3 Übergeordnete Gestaltungsoptionen für ein nachhaltiges

Landnutzungsmanagement .......................... 346

5.2.4 Institutionelle Gestaltungsoptionen im Siedlungswesen . . . . . . . . . . . . 353

5.2.4.1 Beiträge zum Klimaschutz durch die Reduzierung der

Flächeninanspruchnahme ....................... 354

5.2.4.2 Ausgewählte Maßnahmen zur Klimaanpassung im Sektor Siedlung

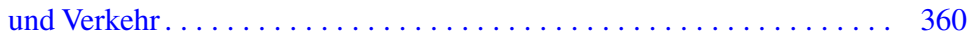

5.2.5 Institutionelle Gestaltungsoptionen bei der landwirtschaftlichen Landnutzung . . 362

5.2.5.1 Nutzung organischer Böden . . . . . . . . . . . . . . . 363

5.2.5.2 Anpassung des Düngemanagements................ 365

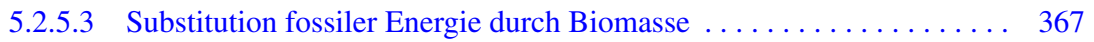

5.2.5.4 Erhaltung und Schaffung von Treibhausgassenken . . . . . . . . . . . 369

5.2.6 Institutionelle Gestaltungsoptionen im Sektor Forstwirtschaft . . . . . . . . . . 370

5.2.6.1 Übergeordnete Gestaltungsoptionen im Sektor Forstwirtschaft . . . . . . 370

5.2.6.2 Gestaltungsoptionen zur Erhöhung der Klimaschutzleistung der Wälder. . . . . . . . . . . . . . . . . . . . . . . . . 371

5.2.6.3 Anpassung der Wälder an den Klimawandel und Vermeidung von Kohlenstoffverlusten............................ 373

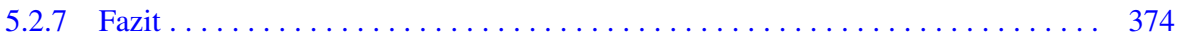

5.3 Landnutzung und Klimawandel im transdisziplinären Diskurs: Wissenschaft und

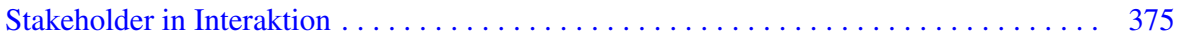

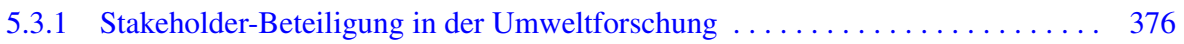

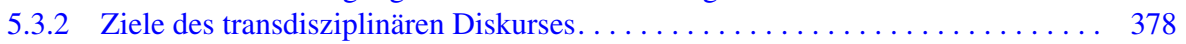

5.3.3 Wissenschaft und Praxis in Interaktion: der Prozess . . . . . . . . . . . . . . 381

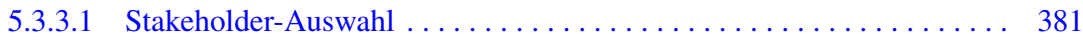

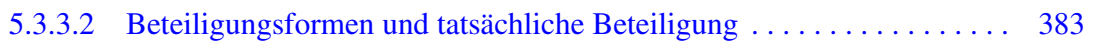

5.3.3.3 Feedbackschleifen. . . . . . . . . . . . . . . . . . . . . . . . . . 389

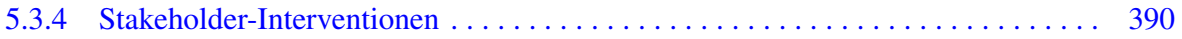

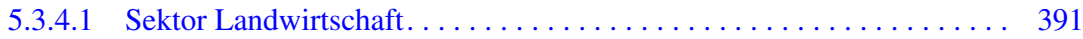

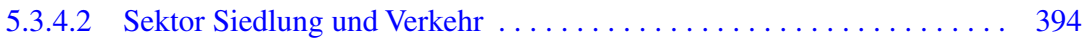

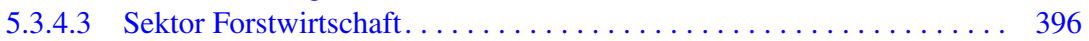

5.3.5 Wissenschaft und Praxis in Interaktion: Prozessreflexionen . . . . . . . . . . . . . 398

5.3.5.1 Der transdisziplinäre Diskurs aus Sicht der Stakeholder . . . . . . . . . 398

5.3.5.2 Der transdisziplinäre Diskurs aus Sicht des Konsortiums . . . . . . . . 403 
5.3.6 Der transdisziplinäre Diskurs: Was hat er gebracht? . . . . . . . . . . . . 405

5.3.6.1 Inhaltliche Aspekte des transdisziplinären Diskurses . . . . . . . . . . . . 405

5.3.6.2 Methodische Aspekte des transdisziplinären Diskurses . . . . . . . . . . . 408

5.3.6.3 Prozessuale Aspekte . . . . . . . . . . . . . . . . . . . . . . . . . 410

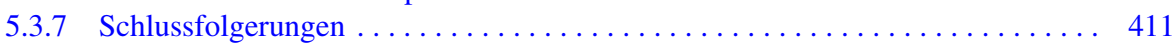

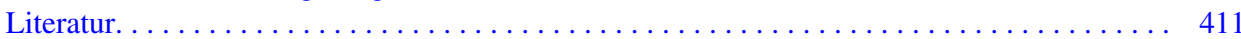

\section{1 Ökonomische Bewertung von Ökosystemleistungen der Landnutzung}

Jesko Hirschfeld, Priska Weller, Julian Sagebiel, Sandra Rajmis und Peter Elsasser

\section{Zusammenfassung}

Landnutzungsänderungen monetäre Werte zuzuweisen ist oft schwierig, da einerseits eine Vielzahl an Akteuren betroffen ist (u. a. Anwohner, Landwirte, Forstwirte, Touristen), andererseits einige Elemente der Landnutzung keinen direkt beobachtbaren monetären Wert aufweisen (Erholungsnutzen, $\mathrm{CO}_{2}$-Speicherung). In diesem Kapitel wird das Konzept der Ökosystemleistungen genutzt, um die verschiedenen Effekte von Landnutzungsänderungen systematisch $\mathrm{zu}$ erfassen und anschließend monetär $\mathrm{zu}$ bewerten. Dabei werden verschiedene ökonomische Methoden (Vermeidungskostenansatz, Choice-Experimente, Änderungen der Nettowertschöpfung zu Faktorkosten) angewendet, die dann in einem Bewertungsrahmen zusammengeführt werden. Schließlich werden die Ergebnisse der ökonomischen Bewertung in eine erweiterte Kosten-Nutzen-Analyse integriert und die in Kap. 4 erarbeiteten Strategien monetär bewertet. Die Bewertung wurde separat für landwirtschaftliche und forstwirtschaftliche Ökosystemleistungen durchgeführt.

Die in Kap. 4 umrissenen Landnutzungsstrategien haben zahlreiche Auswirkungen auf Ökosysteme und ihre für den Menschen relevanten Leistungen. Die verschiedenen Ökosystemleistungen (vgl. Abschn. 5.1.1) können in ihrer Menge oder Qualität mit naturwissenschaftlichen Methoden gemessen und quantifiziert werden - allerdings nur auf jeweils sehr unterschiedlichen Dimensionen. Um die jeweilige Relevanz dieser Daten für die menschliche Gesellschaft bewerten zu können, bedarf es häufig Expertenwissens und ist daher für Laien zumeist schwierig einzuschätzen. Eine Möglichkeit, die verschiedenen Dimensionen einfacher miteinander vergleichbar zu machen, liegt in ihrer ökonomischen Bewertung. Eine Vielzahl ökonomischer Bewertungsmethoden steht zur Verfügung, um den Wert der verschiedenen Ökosystemleistungen für die menschliche Gesellschaft in Geldwerte zu übersetzen - eine Vorgehensweise, die unter wissenschaftlichen, politischen und ethischen Gesichtspunkten jedoch nicht unumstritten ist (Hirschfeld und Sagebiel 2014; Unmüssig 2015; Hansjürgens 2015). Die ökonomische Forschung sozialökologischer Prägung bemüht sich, naturwissenschaftliche Erkenntnisse über Wirkungszusammenhänge und Informationen zu gesellschaftlichen Präferenzen im Hinblick auf die Wertschätzung der verschiedenen Ökosystemleistungen in der monetären Bewertung so weit wie möglich zu berücksichtigen. Die Abbildung 
des Wertes der verschiedenen Ökosystemleistungen in Geldeinheiten soll es im Kontext der bearbeiteten Fragestellungen ermöglichen, die Auswirkungen verschiedener Landnutzungsstrategien untereinander leichter vergleichbar zu machen, um die Abwägung ihrer jeweiligen Vor- und Nachteile für Ökosysteme und Gesellschaft zu vereinfachen.

Bereitstellende Ökosystemleistungen - wie beispielsweise die Produktion von Weizen oder Holz - können über Marktbeobachtungen bewertet werden. Hierzu werden die Ergebnisse aus der Modellierung des Agrar- und Forstsektors in Kap. 4 einbezogen. Relevante regulierende Ökosystemleistungen sind in diesem Fall die Kompensation von THG-Emissionen sowie Nährstoffeinträge in Grundwasser und Oberflächengewässer. Der Wert kultureller Ökosystemleistungen kann nur schwierig monetarisiert werden, da die meisten kulturellen Leistungen nicht auf Märkten gehandelt werden. Wir haben daher eine deutschlandweite, repräsentative Befragungsstudie durchgeführt, mit der die hypothetischen Zahlungsbereitschaften der deutschen Bevölkerung für eine Vielzahl relevanter kultureller Ökosystemleistungen erhoben wurden.

\subsection{1 Überblick über Daten und Methoden zur ökonomischen Bewertung von Ökosystemleistungen}

Jesko Hirschfeld, Julian Sagebiel und Sandra Rajmis

Das Millennium Ecosystem Assessment (2005) systematisiert diese vielfältigen Ökosystemleistungen in die vier Kategorien der bereitstellenden, regulierenden, kulturellen und unterstützenden Ökosystemleistungen (siehe Abb. 5.1). Bereitstellende Ökosystemleistungen umfassen alle direkt nutzbaren Produkte des Ökosystems, wie zum Beispiel Weizen oder Holz. Regulierende Ökosystemleistungen bezeichnen weniger direkt nutzbare Leistungen, die aber, wie die oben beschriebene Kohlenstoffbindung, einen wichtigen Beitrag zur Regulierung des Klimas, des Nährstoff- oder Wasserhaushaltes leistet. Kulturelle Ökosystemleistungen hingegen beziehen sich auf ästhetische, ideelle oder der Erholung dienende Leistungen, wie das Landschaftsbild, die Möglichkeit, bestimmte Arten in freier Wildbahn zu beobachten oder auch nur das Wissen um ihr

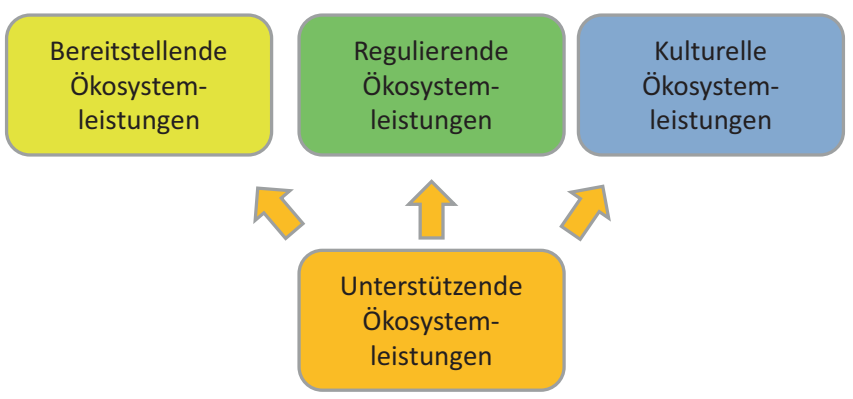

Abb. 5.1 Unterteilung der Ökosystemleistungen und Bewertungsansätze 
Vorhandensein. Die unterstützenden Ökosystemleistungen werden in der hier vorgenommenen ökonomischen Bewertung nicht einbezogen, da sie ihrer Definition nach in die Bereitstellung der anderen Ökosystemleistungen eingehen und somit bei gesonderter Bewertung die Gefahr einer Doppelzählung bestünde.

Ökosystemleistungen sind zunächst in ihren physischen Ausprägungen zu erfassen, beispielsweise in Tonnen geernteter Weizen oder im Boden festgelegte Tonnen Kohlenstoff pro Hektar oder die Anzahl beobachteter Brutpaare bestimmter Vogelarten in einer Region. Die untersuchten Strategien zur nachhaltigen Landnutzung führen zu jeweils unterschiedlichen Auswirkungen auf die verschiedenen Ökosystemleistungen. Sollen nun die Effekte der verschiedenen Strategien untereinander abgewogen werden, um die aus Sicht des Betrachtenden ,beste“ Strategie zu identifizieren, kann dies mit Hilfe einer Multikriterienanalyse direkt anhand ihrer Effekte auf die einzelnen Ökosystemleistungsdimensionen geschehen. Die Schwierigkeit dieser Bewertungsmethode besteht jedoch darin, die einzelnen und sehr verschiedenen Dimensionen von Ökosystemleistungen hinsichtlich ihrer Relevanz zu gewichten und aus diesen einzelnen Gewichtungen eine Rangfolge der Landnutzungsstrategien abzuleiten.

Eine monetäre Bewertung der Ökosystemleistungen erleichtert den Vergleich und die Abwägung zwischen den Effekten auf die jeweils unterschiedlichen Ökosystemleistungen, indem sie ihnen Geldwerte zuweist. Diese Geldwerte haben alle eine einheitliche Dimension (Euro), in der sie dann unmittelbar untereinander verglichen werden können. Diese „Monetarisierung der Natur“ ist als Bewertungsmethode nicht unumstritten (Unmüssig 2015). Wenn sie verantwortungsvoll durchgeführt und verwendet wird, kann sie jedoch dazu beitragen, im politischen Prozess häufig nachrangig behandelten Ökosystemleistungen, wie der Bewahrung der Biodiversität oder des Landschaftsbildes, einen angemesseneren Stellenwert zu verschaffen (Hirschfeld und Sagebiel 2014).

In den folgenden Abschn. 5.1.1.1 bis Abschn. 5.1.1.3 werden zunächst die Methoden zur ökonomischen Bewertung der verschiedenen Kategorien von Ökosystemleistungen vorgestellt. Auf dieser Grundlage werden dann in den Abschn. 5.1.2 und 5.1.3 die Ökosystemleistungen auf landwirtschaftlichen Flächen und auf Waldflächen bewertet. Abschn. 5.1.4 stellt dann die ökonomische Gesamtbewertung der Landnutzungsstrategien vor.

\subsubsection{Bereitstellende Ökosystemleistungen}

Jesko Hirschfeld

Die hier bewerteten bereitstellenden Ökosystemleistungen sind vor allem Produkte der land- und forstwirtschaftlichen Betriebe, wie Weizen, Energiemais oder Holz. Da diese auf Märkten gehandelt werden, können die Kosten und Nutzen, die durch veränderte Landnutzung entstehen, mit Konsumenten- und Produzentenrenten abgebildet werden. Die Konsumentenrente ist die Differenz zwischen Zahlungsbereitschaft der Konsumenten (oder Nachfrager) und dem Marktpreis (p'), die Produzentenrente die Differenz zwischen Produktionskosten und Marktpreis, jeweils aggregiert über die produzierte Menge.

Führt beispielsweise eine Maßnahme zu einer Erhöhung der Produktionskosten, verschiebt sich die Angebotskurve nach oben - ein neues Gleichgewicht mit einem höheren 
Preis und einer geringeren Produktionsmenge entsteht (bei Produktionsmenge x' und Marktpreis p'). Entsprechend ändern sich die Konsumenten- und Produzentenrenten. Die Differenz zwischen Konsumenten- und Produzentenrenten vor und nach der Maßnahme stellt dann die gesellschaftlichen Kosten (bzw. den Nutzenverlust) einer Maßnahme in der Dimension dieser bereitstellenden Ökosystemleistung (z. B. Einbuße an produzierter Maismenge) dar.

Die Berechnung von Konsumenten- und Produzentenrenten setzt voraus, dass die Nachfrage- und Angebotskurven bekannt sind. In vielen Fällen kann man diese jedoch nicht genau bestimmen. In dieser Analyse werden daher die Angebotskurven über die im regionalisierten Agrarsektormodell RAUMIS verwendeten Grenzkostenfunktionen (Abschn. 4.2.2.2, 4.5) approximiert, aus denen im Zusammenspiel mit dem Preisvektor die Nettowertschöpfung abgeleitet wird. Da die analysierten Politikmaßnahmen vor allem die Angebotsseite beeinflussen, nehmen wir die Nachfragefunktion jeweils als konstant an.

Abb. 5.2 zeichnet ein - allerdings stark vereinfachtes - Bild des Marktes für Mais. Tatsächlich sind die Agrarmärkte international, und eine Änderung der inländischen Angebotsfunktion hat eine nur sehr geringe Auswirkung auf den für die Abnehmer wirksamen Marktpreis, da die inländisch ausfallende Angebotsmenge durch Importe aus dem Ausland substituiert werden kann. Daher geht in einem solchen Fall nur die Produzentenrente zurück, während die Konsumentenrente nahezu konstant bleibt.

Aus diesem Grund können die Wohlfahrtswirkungen der landwirtschaftbezogenen Maßnahmen mit den jeweiligen Differenzen der Produzentenrenten approximiert

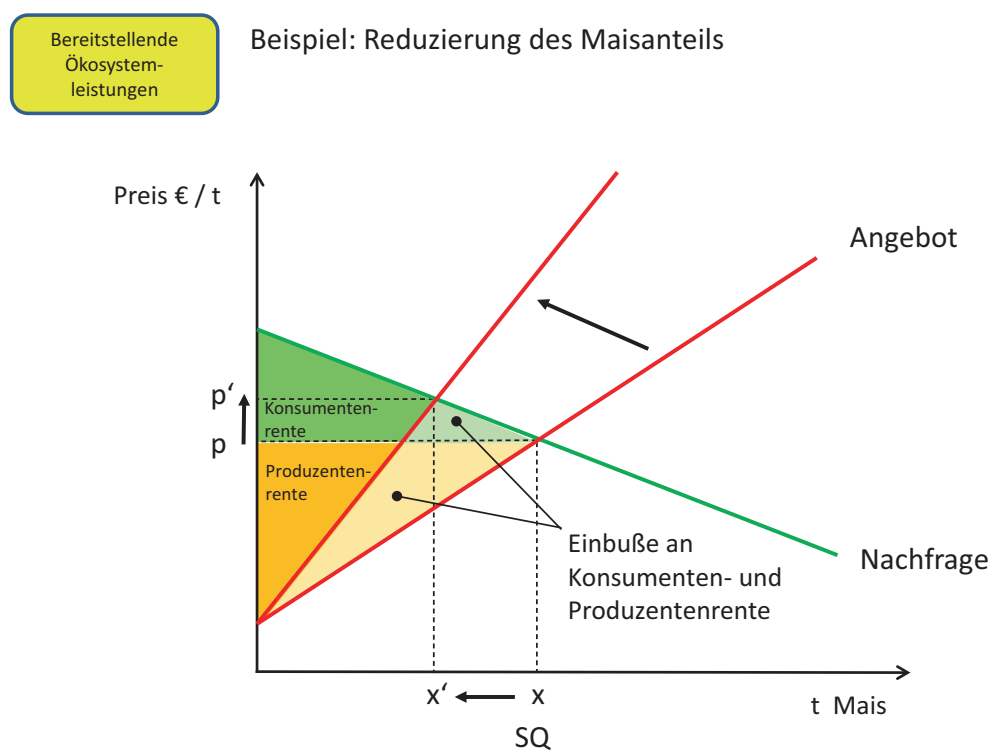

Abb. 5.2 Änderung der Konsumenten- und Produzentenrenten auf dem Markt für Mais bei einer Begrenzung des Maisanteils auf landwirtschaftlichen Flächen 
werden, die der mit dem RAUMIS-Modell ermittelten Differenz der landwirtschaftlichen Nettowertschöpfung entspricht. So ergeben sich beispielsweise bei der Modellierung von Maßnahmen der Strategie „Klimaschutz“ Kosten in der landwirtschaftlichen Produktion (wie in Kap. 4 umrissen) in Form von Einbußen landwirtschaftlicher Nettowertschöpfung von über 1,7 Mrd. €/Jahr. Um gesamtgesellschaftlich wohlfahrtssteigernd zu wirken, müsste eine solche Strategie also in einem anderen Bereich der Ökosystemleistungen (beispielsweise durch verbesserte Klimaregulation oder verringerte Nährstoffausträge) gesellschaftliche Nutzen erzeugen, die mit über 1,7 Mrd. €/Jahr zu bewerten sind. Dies wird in den folgenden Schritten untersucht.

\subsubsection{Regulierende Ökosystemleistungen}

Jesko Hirschfeld

Regulierende Ökosystemleistungen - wie die Regulierung von Nährstoffflüssen, THG-Emissionen oder der Wasserrückhalt im Boden - können durch verschiedene kostenbasierte Methoden ökonomisch bewertet werden, die im Folgenden kurz vorgestellt werden.

Reguliert beispielsweise eine natürliche Auenlandschaft durch die Bereitstellung von Überflutungsflächen den Wasserstand eines Flusses im Hochwasserfall, kann diese Ökosystemleistung durch die vermiedenen Schäden der Flussanlieger flussabwärts monetär bewertet werden (Schadenskostenansatz).

Wird durch eine Extensivierung der landwirtschaftlichen Bewirtschaftung weniger Stickstoff in Grundwasser und Oberflächengewässer ausgetragen, kann dies mit den an anderer Stelle eingesparten Kosten zum Erreichen des guten chemischen Gewässerzustandes bewertet werden (Vermeidungskostenansatz). Vermeidungskosten können als Näherungswert für die gesellschaftliche Zahlungsbereitschaft für die Reduzierung von Beeinträchtigungen von Ökosystemleistungen betrachtet werden. Dabei wird davon ausgegangen, dass der politische Prozess individuelle Präferenzen soweit adäquat abbildet und in umweltpolitische Rahmensetzungen übersetzt, dass die damit verbundenen Kosten von einer deutlichen Mehrheit als angemessen empfunden werden (Schroeder 2010; Oates und Portney 2003; Aidt 1998). Der Vermeidungskostenansatz erfasst also diejenigen Kosten, die an anderer Stelle entstehen, um einen durch die Beeinträchtigung einer Ökosystemdienstleistung entstandenen negativen Effekt (z. B. Eutrophierung, Treibhauswirkung) wieder aufzuwiegen.

Beim Ersatzkostenansatz dagegen wird nach einem unmittelbaren Ersatz der Ökosystemleistung selber gesucht. Wird beispielsweise die Wasserrückhaltefunktion einer Flussaue durch den Bau eines Deiches eingeschränkt, kann zum Ausgleich ein Polder oder ein Hochwasserrückhaltebecken angelegt werden, der eine äquivalente Funktion erfüllt und die ursprüngliche, natürliche Ökosystemleistung der Aue ersetzt. Das von einer versiegelten Fläche abgeleitete Regenwasser kann auf einer angrenzenden Fläche mit Hilfe einer Mulde oder eines Schachtes gezielt versickern, wodurch versucht wird, die ursprüngliche natürliche Versickerungsleistung beispielsweise einer Wiese durch 
eine über technische Infrastrukturen vermittelte Versickerung zu ersetzen. Ein Vorteil des Ansatzes besteht darin, dass Marktpreise des Substitutes für die Bewertung verwendet werden können (Bräuer und Marggraf 2004). Ein kritischer Punkt dieser Methode liegt in der Frage, inwiefern eine bestimmte Ökosystemleistung durch das betreffende Ersatzgut tatsächlich vollständig zu substituieren ist, d. h., ob der Nutzen annähernd derselbe bleibt - denn der Gesamtwert einer Wiese oder einer Flussaue liegt nicht allein in der Versickerungs- oder Wasserrückhaltewirkung, sondern beispielsweise auch in der Bewahrung der biologischen Vielfalt und in der Bereitstellung von Erholungsleistungen.

Bei der monetären Bewertung der näher untersuchten regulierenden Ökosystemleistungen „Regulation von Kohlendioxid und anderen Treibhausgasen“ sowie „Regulation des Stickstoffkreislaufs“ wird hier der Vermeidungskostenansatz verwendet. Diese Auswahl wurde getroffen, um eine größtmögliche methodische Kohärenz der in den drei untersuchten Ökosystemleistungskategorien ermittelten monetären Werte zu erreichen.

\subsubsection{Kulturelle Ökosystemleistungen}

Priska Weller, Julian Sagebiel und Sandra Rajmis

Kulturelle Ökosystemleistungen sind im Allgemeinen keine Güter, die auf Märkten gehandelt werden. Deshalb haben sie keinen Preis, der für ihre Bewertung herangezogen werden könnte. Daher werden zur Bewertung von kulturellen Ökosystemleistungen mit Hilfe von Befragungen offenbarte bzw. geäußerte Präferenzen erhoben. Offenbarte Präferenzen werden u. a. über Marktpreise und Reisekosten erfasst. Geäußerte Präferenzen werden zum Beispiel durch Kontingente Bewertungen sowie Auswahlexperimente („Choice-Experimente“) erhoben, in denen eine repräsentativ ausgewählte Stichprobengruppe anhand von strukturierten Interviews befragt wird. Daraus werden Zahlungsbereitschaften (ZB) abgeleitet.

Choice-Experimente sind eine mittlerweile etablierte Methode zur Bewertung von Landschafts- und Landnutzungsänderungen (van Zanten et al. 2014). Die Methode eignet sich vor allem, weil einzelne Ökosystemleistungen individuell bewertet werden können. So kann beispielsweise eine individuelle Zahlungsbereitschaft für einen höheren Waldanteil an der Landschaft einer Zahlungsbereitschaft für einen geringeren Maisanteil gegenübergestellt werden. Weiterhin erlaubt die Methode, aggregierte Zahlungsbereitschaften für Maßnahmenbündel zu berechnen, die sich auf mehrere Ökosystemleistungen gleichzeitig auswirken.

Choice-Experimente sind meist in einen größeren Fragebogen eingebettet, in dem z. B. auch soziodemographische Merkmale abgefragt werden, die später zur Auswertung des Choice-Experiments herangezogen werden können. Beispielsweise kann dadurch die Auswertung auf eine bestimmte Altersgruppe oder auf eine bestimmte Befragungsregion fokussiert werden. Im Choice-Experiment entscheiden sich die Befragten wiederholt 
zwischen verschiedenen Landnutzungsszenarien. Die Szenarien werden durch verschiedene Merkmale oder Attribute, die die Landnutzung beschreiben, dargestellt und unterscheiden sich in den Ausprägungen der Merkmale. Nimmt man ein Preismerkmal hinzu, kann man aus dem Abwägungsverhältnis zwischen einer zusätzlichen Einheit des Landschaftsmerkmals und einer zusätzlichen Preiseinheit Zahlungsbereitschaften berechnen. Bei einer Verbesserung eines Merkmals um eine Einheit ist der Befragte bereit, einen bestimmten Eurobetrag zu zahlen, ohne dass er besser oder schlechter gestellt wird - das Nutzenniveau bleibt konstant. Die einzelnen Befragten bekommen in der Regel zwischen vier und zwölf solcher Entscheidungssituationen vorgelegt, um eine hohe Anzahl an Beobachtungen zu erzielen. Die Zusammenstellung der Merkmalsausprägungen im Choice-Experiment basiert auf einem experimentellen Design. Ein gutes experimentelles Design lässt eine unabhängige Schätzung des Einflusses einzelner Merkmale auf die Entscheidung zu.

Mit Regressionsverfahren, insbesondere statistischen Antwortmodellen, können die Ergebnisse der Bevölkerungsbefragung ausgewertet werden. In der Literatur wird häufig das multinominale Logit-Model genutzt. Dieses geht davon aus, dass die Präferenzen der Befragten stets homogen sind. Um realitätsnähere heterogene Präferenzen zu berücksichtigen, wurden in den letzten 20 Jahren Erweiterungen des Logit-Models entwickelt.

\section{Datenerhebung}

Zur Bewertung der kulturellen Ökosystemleistungen der Landschaft wurde im Frühjahr 2013 eine große deutschlandweite Bevölkerungsbefragung durchgeführt. Die Befragten wurden aus der Adressdatei eines Befragungsinstituts gezogen. Quotiert wurde nach den Merkmalen Alter, Geschlecht, Bundesland, gefiltert nach privater Internetnutzung. Die Antwortrate betrug ca. $30 \%$ der kontaktierten Panel-Teilnehmer, 8800 Fragebögen wurden vollständig beantwortet. Die Stichprobe ist bezüglich der für die Quotierung ausgewählten Merkmale weitgehend repräsentativ für die Gesamtbevölkerung Deutschlands. Einkommen und Bildungsstand der Befragten sind jedoch etwas überdurchschnittlich.

Für die Online-Befragung wurde ein Fragebogen mit sechs unterschiedlichen Versionen des Choice-Experiments erstellt. Die Befragten wurden zufällig auf die sechs verschiedenen Choice-Experiment-Versionen verteilt. Der restliche Fragebogen war bei allen Befragten identisch. Im Choice-Experiment gab es die drei unterschiedlichen Themenschwerpunkte „Wald“, „landwirtschaftlich genutzte Landschaft" und „Landschaft allgemein (ohne besonderen forst- oder landwirtschaftlichen Schwerpunkt)“, jeweils unterteilt in mehrere Merkmale. Pro Thema gab es jeweils zwei Fragebogenversionen. Vor dem Choice-Experiment wurden Fragen zur Landschaftsnutzung der Befragten (Freitzeitnutzung der Umgebung) gestellt sowie eine Einschätzung des jeweiligen aktuellen Zustands der wohnortnahen Landschaft erbeten. Nach dem ChoiceExperiment wurden Landschaftspräferenzen und Einstellungen zu Landnutzung erfragt. Am Ende des Fragebogens wurden soziodemographische Merkmale der Befragten erhoben. Zuletzt wurden die Befragten gebeten, auf einer eingeblendeten Landkarte ihren Wohnort zu markieren, um bei der Auswertung des Choice-Experiments die 
Wenn nur die folgenden Möglichkeiten für die Entwicklung der Landschaft im Umkreis von bis zu 15 Kilometern um den Ort, an dem Sie wohnen, zur Verfügung stünden, welche würden Sie wählen?

Wenn Sie in einer großen Stadt wohnen, denken Sie bitte an die Umgebung dieser Stadt.

\begin{tabular}{|c|c|c|c|}
\hline & Landschaft A & Landschaft B & Landschaft C \\
\hline Anteil Wald an der Landschaft & $10 \%$ niedriger & $10 \%$ höher & Wie heute \\
\hline Größe einzelner Felder und Waldstücke & Halb so groß & Doppelt so groß & Wie heute \\
\hline Waldflächen mit Unterwuchs & Halb so viele & Doppelt so viele & Wie heute \\
\hline Nadelbaumanteil & $30 \%$ Nadelbaumanteil & $70 \%$ Nadelbaumanteil & Wie heute \\
\hline Erntealter der Wälder & 20 Jahre später ernten & 30 Jahre später ernten & Wie heute \\
\hline (@) Finanzieller Jahresbeitrag zum Landschaftsfonds & $25 €$ & $80 €$ & $0 €$ \\
\hline Ich wähle... & 0 & 0 & 0 \\
\hline
\end{tabular}

Abb. 5.3 Beispiel für eine Choice-Karte

individuelle Landschaftsumgebung einzubeziehen. Vor der Durchführung der Interviews wurde der Fragebogen in Fokusgruppengesprächen geprüft, indem er Personen aus verschiedenen Alters-, Bildungs- und Berufsgruppen vorgelegt und auf Verständlichkeit und Relevanz getestet wurde.

Das Choice-Experiment war so strukturiert, dass mehrere Landschaftsmerkmale mit verschiedenen Ausprägungen spaltenweise zu „Landschaften“ kombiniert und den Befragten zur Auswahl gegeben wurden. Die Landschaftsmerkmale wurden bei der Erstellung der verschiedenen Auswahlkarten beibehalten, die Ausprägungen aber verändert, sodass sich pro Befragtem nacheinander neun unterschiedliche Auswahlentscheidungen ergaben. Die Befragten wurden gebeten, aus den jeweils drei vorgeschlagenen „Landschaften“ auf jeder Auswahlkarte die Landschaft A, B oder C auszuwählen (siehe Abb. 5.3). Diese Auswahl sollte in Bezug auf einen Umkreis von ca. $15 \mathrm{~km}$ um den jeweiligen individuellen Wohnort getroffen werden. Das Choice-Experiment in jeder Fragebogenversion bestand aus fünf Landschaftsmerkmalen und zusätzlich einem Preismerkmal zur Ermittlung der individuellen Zahlungsbereitschaft. Dieses Preismerkmal wurde konkret als „Finanzieller Jahresbeitrag zum Landschaftsfonds“ ausgestaltet. Zwei der fünf Landschaftsmerkmale waren aus methodischen Gründen in allen sechs Fragebogenversionen gleich, ebenso das Preismerkmal. Die verbleibenden drei Merkmale definierten die unterschiedlichen Themenschwerpunkte und unterschieden sich in den Versionen. Die Reihenfolge, in der die Merkmale visuell nacheinander präsentiert wurden, war immer identisch, weiterhin war die dritte präsentierte „Landschaft C“ immer die sogenannte Status quo-Alternative, in der sich nichts zum heutigen Zustand ändert. Alle Merkmale wurden im Fragebogen mit kleinen Piktogrammen illustriert, um die Unterscheidung zu erleichtern. Zusätzlich wurde jedes Merkmal mit einem kurzen Text erläutert (20 bis 30 Wörter), den die Befragten mit Hilfe eines Info-Buttons jederzeit während des Choice-Experiments aufrufen konnten. Abb. 5.3 ist 
ein Beispiel für eine Auswahlkarte, wie sie für die Befragten auf dem Bildschirm sichtbar war.

Der nachfolgende Abschnitt befasst sich mit der Erläuterung der beiden Themenschwerpunkte „Wald“ und „Landwirtschaft“. In allen Fragebogenversionen waren die Merkmale „Anteil Wald an der Landschaft“ und „Größe einzelner Felder und Waldstücke“ sowie das Preismerkmal „Finanzieller Jahresbeitrag zum Landschaftsfonds“ enthalten. Im Themenschwerpunkt „Wald“ kamen die Merkmale „Waldflächen mit Unterwuchs“, „Nadelbaumanteil“ und „Erntealter der Wälder“ (Version 1) bzw. „Artenvielfalt im Wald“, „Anteil ungenutzter Waldflächen“ und „Anteil von Bäumen aus anderen Ländern“ (Version 2) hinzu. Im Themenschwerpunkt „Landwirtschaft“ wurden die Merkmale „Landwirtschaftliche Flächen mit hohem Naturwert“, „Heckenanteil an Acker- und Wiesenrändern“ und „Fläschenverbrauch“ (Version 3) bzw. „Artenvielfalt auf landwirtschaftlichen Flächen“, „Maisanteil“ und „Anteil von Wiesen und Weiden“ (Version 4) zusätzlich aufgenommen. Manche der Merkmale haben Entsprechungen in den Fragebogenversionen zu den anderen Landschaftstypen, z. B. Artenvielfalt im Wald und Artenvielfalt auf landwirtschaftlichen Flächen (Tab. 5.1).

\subsubsection{Ergebnisse der ökonomischen Bewertung der Ökosystemleistungen landwirtschaftlicher Landnutzungen}

Jesko Hirschfeld und Julian Sagebiel

\subsubsection{Bereitstellende Ökosystemleistungen landwirtschaftlicher Landnutzungen}

Jesko Hirschfeld

Die alternativen Strategien für eine nachhaltige Landnutzung mit den unterschiedlichen Fokussierungen auf Klimaschutz, Erzeugung von Biomasse und Umwelt- und Naturschutz haben Auswirkungen auf die durch die Landwirtschaft bereitstellbaren Mengen an Nahrungsmitteln und Biomasse. Die nachfolgend dargestellten Auswirkungen der unterschiedlichen Rahmensetzungen auf diese bereitstellenden Ökosystemleistungen sind mit Hilfe des RAUMIS-Modells abgebildet worden (vgl. Kap. 4). Alle drei untersuchten Strategien bewirken eine Reduzierung der Produktion von Nahrungsmitteln und einen Zuwachs an Biomasseproduktion jeweils unterschiedlicher Biomassearten (je nach Strategie Holz, Paludikulturen, Energiemais, alternative Energiepflanzen oder Grünland).

Für die ökonomische Bewertung dieser Produktionswirkungen stellt RAUMIS die regionale „Nettowertschöpfung zu Faktorkosten“ als Sektoreinkommen bereit. Die Differenzen zwischen der landwirtschaftlichen Nettowertschöpfung bei Umsetzung der jeweiligen Strategie und der Nettowertschöpfung in der Referenzprojektion 2030 werden hier als gesellschaftliche Kosten der jeweiligen Strategien interpretiert und in Tab. 5.5 in die erweiterte ökonomische Gesamtbewertung der Strategien einbezogen. 
Tab. 5.1 Merkmale und ihre Ausprägungen. (Quelle: J. Hirschfeld, P. Weller, J. Sagebiel, S. Rajmis, P. Elsasser)

\begin{tabular}{|c|c|c|}
\hline Fragebogenversion & Merkmale & $\begin{array}{l}\text { Ausprägungen } \\
\text { (Basis: wie heute) }\end{array}$ \\
\hline \multirow[t]{3}{*}{ Alle } & Anteil Wald an der Landschaft & $10 \%$ niedriger, $10 \%$ höher \\
\hline & Größe einzelner Felder und Waldstücke & $\begin{array}{l}\text { Halb so groß, } \\
\text { Doppelt so groß }\end{array}$ \\
\hline & $\begin{array}{l}\text { Finanzieller Jahresbeitrag zum Land- } \\
\text { schaftsfonds (€/a) }\end{array}$ & $0,10,25,50,80,110,160$ \\
\hline \multirow[t]{3}{*}{ Version 1} & Waldflächen mit Unterwuchs & Halb so viele, doppelt so viele \\
\hline & Nadelbaumanteil & $\begin{array}{l}30 \% \text { Nadelbaumanteil, } \\
70 \% \text { Nadelbaumanteil }\end{array}$ \\
\hline & Erntealter der Wälder & $\begin{array}{l}20 \text { Jahre später ernten, } \\
30 \text { Jahre später ernten }\end{array}$ \\
\hline \multirow[t]{3}{*}{ Version 2} & Artenvielfalt im Wald & $\begin{array}{l}\text { Leicht erhöhen (85 Punkte), } \\
\text { Deutlich erhöhen (105 } \\
\text { Punkte) }\end{array}$ \\
\hline & Anteil ungenutzter Waldflächen & $\begin{array}{l}0 \% \text { der Waldflächen, } \\
10 \% \text { der Waldflächen }\end{array}$ \\
\hline & Anteil von Bäumen aus anderen Ländern & $\begin{array}{l}\text { Halb so hoch, } \\
\text { Doppelt so hoch }\end{array}$ \\
\hline \multirow[t]{3}{*}{ Version 3} & $\begin{array}{l}\text { Landwirtschaftliche Flächen mit hohem } \\
\text { Naturwert }\end{array}$ & $\begin{array}{l}\text { Anteil verdoppeln, } \\
\text { Anteil verdreifachen }\end{array}$ \\
\hline & $\begin{array}{l}\text { Heckenanteil an Acker- und Wiesen- } \\
\text { rändern }\end{array}$ & $50 \%, 90 \%$ \\
\hline & Flächenverbrauch & $\begin{array}{l}\text { Flächenverbrauch halbieren, } \\
\text { Flächenverbrauch stoppen }\end{array}$ \\
\hline \multirow[t]{3}{*}{ Version 4} & $\begin{array}{l}\text { Artenvielfalt auf landwirtschaftlichen } \\
\text { Flächen }\end{array}$ & $\begin{array}{l}\text { Leicht erhöhen ( } 85 \text { Punkte), } \\
\text { Deutlich erhöhen (105 } \\
\text { Punkte) }\end{array}$ \\
\hline & Maisanteil & $\begin{array}{l}\text { Max. } 30 \% \text { der Ackerfläche, } \\
\text { Max. } 70 \% \text { der Ackerfläche }\end{array}$ \\
\hline & Anteil von Wiesen und Weiden & $\begin{array}{l}25 \% \text { Wiesen und Weiden, } \\
50 \% \text { Wiesen und Weiden }\end{array}$ \\
\hline
\end{tabular}

Da es für die Bevölkerung nicht trivial ist Artenvielfalt zu bewerten, wurde ein Indikator mit einer Punkteskala verwendet (Artenvielfalt: 85 Punkte, 105 Punkte), der dies erleichtern sollte. Die Bundesregierung hat 2002 in der Nachhaltigkeitsstrategie einen Indikator für „Artenvielfalt und Landschaftsqualität“" entwickelt, der über die Zählung relevanter Vogelarten die Artenvielfalt in sechs verschiedenen Landschaftstypen misst, da diese Vogelarten gleichzeitig Auskunft über das Vorhandensein anderer Tier- und Pflanzenarten geben (Bundesregierung 2002) 
$\mathrm{Zu}$ beachten ist dabei, dass es sich hierbei um eine Bewertung aus gesellschaftlicher Perspektive handelt. Aus betrieblicher Perspektive sind noch die Transferzahlungen $\mathrm{zu}$ berücksichtigen, die entsprechend der Ausgestaltung der Szenarien vorgesehen sind - wie beispielsweise Prämien für die Mitigationsleistungen. Dadurch fällt die ökonomische Bewertung aus gesellschaftlicher und aus betrieblicher Perspektive auseinander. Insbesondere die Bioenergiestrategie ist unter Einschluss der Mitigationsprämien aus sektoraler Sicht (betriebswirtschaftlich) sehr vorteilhaft.

Aus volkswirtschaftlicher Sicht ergeben sich bei konventioneller ökonomischer Bewertung für alle drei Strategien auf der Betrachtungsdimension der bereitstellenden Leistungen negative Effekte - diese sind bei der Bioenergiestrategie am geringsten (-141 Mio. € gegenüber $-1,4 \mathrm{Mrd}$ € bei der Natur- und Umweltschutzstrategie und $-1,7 \mathrm{Mrd}$. $€ / J a h r$ bei der Klimaschutzstrategie). Für die erweiterte ökonomische Gesamtbewertung der Strategien müssen jedoch neben diesen ökonomischen Effekten im engeren Sinne auch die Effekte auf die regulierenden und kulturellen Ökosystemleistungen einbezogen werden (vgl. dazu die nachfolgenden Abschnitte und Tab. 5.5).

\subsubsection{Regulierende Ökosystemleistungen landwirtschaftlicher Landnutzungen}

Jesko Hirschfeld und Julian Sagebiel

Zur Erhebung der Kosten der Vermeidung von Stickstoff- und THG-Emissionen wurde eine umfangreiche Recherche durchgeführt, in deren Zuge vorliegende wissenschaftliche Studien, Veröffentlichungen von Verbänden und Umweltbehörden sowie technische Planungsdaten mit Kostenansätzen ausgewertet wurden. Auf dieser Grundlage wurden Spannbreiten für die jeweiligen Vermeidungskosten ermittelt, die (vgl. Abschn. 5.1.4) in die monetäre Gesamtbewertung der unterschiedlichen Landnutzungsstrategien einbezogen werden.

Klimaschutz ist der Bevölkerung sehr wichtig. Nach einer aktuellen repräsentativen Umfrage des Emnid Instituts (AEE 2015) befürworten 93 \% der deutschen Bevölkerung den weiteren Ausbau erneuerbarer Energien - und dies, obwohl sie aktuell bereits erhebliche Kosten der Förderung dieses Technologiewechsels tragen. $82 €$ pro vermiedene Tonne $\mathrm{CO}_{2}$ (insgesamt 7,6 Mrd. €) wurden im Jahr 2009 über das Erneuerbare-EnergienGesetz auf die privaten Haushalte umgelegt ${ }^{1}$ - ein Betrag, der in den Folgejahren noch weiter gestiegen ist. Die Ergebnisse der Emnid-Umfrage aus dem Jahr 2015 legen den Schluss nahe, dass die Obergrenze der Zahlungsbereitschaft zur Vermeidung THGEmissionen trotzdem noch nicht erreicht ist.

Eine Metastudie im Auftrag des Umweltbundesamtes (2012) wertete eine Vielzahl von Studien zur Abbildung der Kosten des Klimawandels und der Vermeidung von Treibhausgasen aus und erstellte eine Übersicht zu den Spannbreiten der Kostenwerte. Dabei

\footnotetext{
${ }^{1}$ Berechnungen von J. Hirschfeld und J. Sagebiel auf Grundlage von ISI, GWS, IZES, DIW
} (2011). 
ergaben sich aktuelle Kosten zwischen 40 und $120 € / t \mathrm{CO}_{2}$, mittelfristig (2030) zwischen 70 und $215 €$ und längerfristig (2050) zwischen 130 und $390 € / t \mathrm{CO}_{2}$ (vgl. Schwermer et al. 2012). Wille et al. (2012) ermittelten für 2010 durchschnittliche Vermeidungskosten von $77 € / t$ (mit einer Spannbreite von 44 bis $135 €$ ). Vor dem Hintergrund dieser Studien zu Schadens- und Vermeidungskosten und der Maßgabe, eine vorsichtige Schätzung vorzunehmen, empfahl das Umweltbundesamt, den Wert von $80 € / t \mathrm{CO}_{2}$ als „,Best-PracticeKostensatz“ zu verwenden (vgl. Umweltbundesamt 2012, S. 5). Dieser Empfehlung sind wir in den in Abschn. 5.1.4 vorgenommenen Berechnungen der Kosten und Nutzen der untersuchten Landnutzungsstrategien für den mittleren Bewertungsansatz gefolgt. Als Mindestwert zur monetären Bewertung wird der Durchschnittswert des Preises für $\mathrm{CO}_{2}$-Zertifikate auf dem europäischen Emissionshandelsmarkt in den Jahren 2010 bis 2015 ( $8 €$ pro Tonne $\mathrm{CO}_{2}$ ) und als Obergrenze der vom Umweltbundesamt (2012, S. 5) empfohlene Wert von $120 €$ pro Tonne $\mathrm{CO}_{2}$ angesetzt. $^{2}$

Bei der Bewertung der Strategien sind neben den direkten Klimaeffekten (z. B. Kohlenstofffestlegung durch Wiedervernässung von Mooren oder durch C-Speicherung in Holzbiomasse bei Aufforstung) auch die indirekten Effekte zu berücksichtigen, die durch die Umsetzung der jeweiligen Strategien ausgelöst werden. So führen Aufforstungsmaßnahmen, die Ausweitung des Anbaus von Energiepflanzen oder Flächenstilllegungen zu einer Reduktion der für den Anbau von Nahrungsmitteln zur Verfügung stehenden Agrarflächen in Deutschland. Bleibt die Nachfrage nach Nahrungsmitteln derweil gleich, müssen mehr Nahrungsmittel importiert werden und führen zu einer Ausweitung der Flächennutzung durch die Landwirtschaft in anderen Ländern, die Nahrungsmittel nach Deutschland exportieren. Die Klimaeffekte dieser indirekten Landnutzungsänderungen müssen bei ganzheitlicher Betrachtung in die Bewertung der Strategien einbezogen werden.

Ein weiterer indirekter Effekt entsteht bei einer Reduzierung des Einsatzes von Mineraldünger: Neben dem direkten Effekt, dass von den gedüngten Flächen weniger Treibhausgase emittiert werden, gehen die Treibhaus-Emissionen indirekt noch weiter

\footnotetext{
${ }^{2}$ In Abschn. 6.1 werden zudem Bewertungsvarianten anhand derzeitiger Preise für $\mathrm{CO}_{2}$-Zertifikate auf dem europäischen Emissionshandelsmarkt gerechnet und erläutert, um zusätzlich den privatnützigen Aspekt der Senkenleistung des Waldes abzubilden. Mittlerweile (im Jahr 2020) hat sich der europäische Zertifikatepreis bei circa $25 €$ pro Tonne $\mathrm{CO}_{2}$ - ̈̈quivalente eingependelt und das Umweltbundesamt empfiehlt einen globalen Schadenskostensatz von $180 €$ pro Tonne $\mathrm{CO}_{2}$ - ̈̈quivalent (Edenhofer et al. 2019; Umweltbundesamt 2019). Wir sind in dieser Veröffentlichung bei den Werten aus den Jahren 2010 bis 2015 geblieben, um die zur Zeit der Befragung herrschenden Marktbedingungen und Werthaltungen durch die Bevölkerung konsistent abzubilden. Aktuell hat sich die Sensibilität gegenüber dem Thema Klimawandel und $\mathrm{CO}_{2}$-Emissionen deutlich erhöht damit mutmaßlich auch die Wertschätzung und Zahlungsbereitschaft zur Reduzierung von Treibhausgasemissionen. Eine Berücksichtigung dieser aktuellen Entwicklungen würde den Wert der Treibhausgasemissionsverringerungen der hier untersuchten Strategien weiter erhöhen.
} 
zurück, weil damit zugleich ein Teil der energie- und treibhausgasemissionsintensiven Produktion von Düngemitteln zurückgeht.

In der ökonomischen Gesamtbewertung der Strategien (vgl. Abschn. 5.1.2.4 und Tab. 5.3) werden diese indirekten Effekte einbezogen.

Neben dem Klimaschutz betreibt die Gesellschaft in Deutschland gegenwärtig außerdem einen erheblichen Aufwand, um den ökologischen Zustand der Oberflächengewässer zu verbessern. In 9632 kommunalen Kläranlagen wurden im Jahr 2014 insgesamt gut 8,4 Mrd. $\mathrm{m}^{3}$ Abwasser gereinigt und dabei mehr als $350.000 \mathrm{t}$ Stickstoff eliminiert (vgl. dazu Grünebaum et al. 2006; DWA 2015). ${ }^{3}$ In einer repräsentativen Umfrage antworteten $61 \%$ der Wassernutzer in Deutschland, der Wasserpreis solle die Umweltauswirkungen des Wassergebrauchs reflektieren (European Commission, Directorate-General for Environment, Flash Eurobarometer 344, „Attitudes of Europeans towards water - related issues“, Mai 2012. Zitiert nach BDEW 2015, S. 129). Dies deutet darauf hin, dass die gegenwärtigen Bemühungen zur Vermeidung von Stickstoffeinträgen in die Gewässer die Präferenzen der Bevölkerung im Hinblick auf den Gewässerschutz zumindest annähernd widerspiegeln. In einer älteren Studie gibt Gren (1995) eine Grenzkostenspannbreite von 3,9 bis 35,1 €/kg N an. Da diese Werte inzwischen über zwanzig Jahre zurückliegen, ist davon auszugehen, dass aufgrund der unterdessen vorgenommenen Investitionen in den Ausbau von Reinigungsstufen der untere Rand dieser Spannbreite nicht mehr realisierbar ist und die aktuell relevanten Grenzkosten daher höher liegen. Darauf deutet auch eine jüngere Studie von Siewert (2010) hin, die für einen weiteren Ausbau der Kläranlagen im deutschen Teil des Oder-Einzugsgebietes Grenzkosten zwischen 15 und $430 € / \mathrm{kg} \mathrm{N}$ (Median bei $142 €$ ) berechnet. Und Mutz et al. (2013) beziffern die Kosten der Vermeidung durch die weitere Aufrüstung von Kläranlagen auf Werte zwischen 3 und $50 € / \mathrm{kg}$ zusätzlich eliminierten Stickstoffs.

Da zahlreiche Kommunen trotz dieser z. T. hohen Kosten in die weitere Aufrüstung der Kläranlagentechnik investieren und dies über die Abwassergebühren refinanzieren, könnte auch der obere Bereich dieser Grenzkosten der Vermeidung als Obergrenze der gesellschaftlichen Zahlungsbereitschaft in die Bewertung einbezogen werden. Theoretisch könnte die tatsächliche Zahlungsbereitschaft der Gesellschaft sogar noch höher liegen - in dem Fall nämlich, in dem der durch die 4. Reinigungsstufe erreichte Reinigungsgrad den ökologischen Zielsetzungen der Bevölkerung noch immer nicht genügt.

$\mathrm{Zu}$ Validierung dieses Bewertungsansatzes können zum Vergleich Zahlungsbereitschaftsstudien herangezogen werden, die eine repräsentative Stichprobe von Befragten direkt nach ihrer Zahlungsbereitschaft für eine Verbesserung der Gewässerqualität gefragt haben. Meyerhoff et al. (2014) ermitteln verlässlich positive NutzenKosten-Verhältnisse für Maßnahmen zur Verbesserung der Gewässerqualität, deren

\footnotetext{
${ }^{3}$ Sie repräsentieren 92,3\% der Einwohnergleichwerte (vgl. Abschn. 6.1).
} 
Vermeidungskosten bei 13 bis $15 € / \mathrm{kg} \mathrm{N}$ liegen. Ab Werten von über $20 € / \mathrm{kg} \mathrm{N}$ steht es je nach Annahme zu den Unsicherheiten in Frage, ob das Nutzen-Kosten-Verhältnisse noch positiv ausfällt.

Im Sinne eines konservativen Bewertungsansatzes, der eine Überbewertung vermeiden möchte, werden zur Bewertung der Stickstoff-Emissionsdifferenzen der verschiedenen Strategien (vgl. Abschn. 5.1.4) die aktuell realisierten Durchschnittskosten der Vermeidung verwendet $(6,30 € / \mathrm{kg} \mathrm{N})$, die von den Bürgerinnen und Bürgern akzeptiert sind und über die Abwassergebühren getragen werden. Als Spannbreite der alternativ möglichen Werte wird für die Sensitivitätsanalyse der untere Wert $(3 € / \mathrm{kg} \mathrm{N})$ aus der Studie von Mutz et al. (2013) und der obere Wert aus der Studie von Meyerhoff et al. (2014) verwendet, der gerade noch ein positives Nutzen-Kosten-Verhältnis garantiert $(15 € / \mathrm{kg} \mathrm{N})$.

Der hier gewählte Ansatz bewegt sich also in einem von den benannten Studien bestätigten Rahmen und kann damit als empirisch gut abgesichert betrachtet werden. Insbesondere kann damit auch mit großer Sicherheit ausgeschlossen werden, dass es sich bei dem für die Gesamtbewertung in Abschn. 5.1.2.4 verwendeten Referenzwert von $6,30 € / \mathrm{kg} N$ um eine Überschätzung der gesellschaftlichen Zahlungsbereitschaft zur Verminderung von Stickstoffeinträgen in die Oberflächengewässer, sondern eher um eine Untergrenze handelt. Die Randwerte der hier herausgearbeiteten Spannbreite werden in Abschn. 5.1.2.4 jedoch zur Einordnung ergänzend berechnet.

\subsubsection{Kulturelle Ökosystemleistungen landwirtschaftlicher Landnutzungen}

Julian Sagebiel und Jesko Hirschfeld

Änderungen der kulturellen Ökosystemleistungen werden mit Hilfe von Wohlfahrtsänderungen, die aus dem landwirtschaftlichen Teil des Choice-Experiments berechnet wurden, bewertet. In einem ersten Schritt wird die funktionale Form der Nutzenfunktion spezifiziert. Die einfachste Spezifikation ist eine lineare Form, d.h., eine Erhöhung eines Attributes um eine Einheit erbringt immer denselben Nutzenzuwachs, unabhängig von der Ausgangssituation. Da von einem linearen Zusammenhang nicht ausgegangen werden kann, ist eine nichtlineare Spezifikation erforderlich. Eine Möglichkeit, nichtlineare Effekte in die Nutzenfunktion zu integrieren, ist die Erstellung von DummyVariablen. Dabei wird für jede Ausprägung eines Attributes eine neue Variable erstellt, die den Wert 1 annimmt, wenn die Ausprägung gegeben ist, und sonst null ist. Das ermöglicht, den Einfluss jeder Ausprägung auf den Nutzen separat zu schätzen. Der Vorteil bei dieser Spezifikation liegt vor allem darin, keine weiteren Annahmen über die funktionale Form treffen zu müssen. Allerdings ist es dann nicht möglich, konkrete Aussagen über den Effekt von Ausprägungen zu treffen, die nicht im Choice-Experiment integriert wurden. Falls das notwendig ist, muss auf parametrische Funktionen zurückgriffen werden. Für die Modellierung der landwirtschaftlichen Landnutzung ist die parametrische Darstellung der Nichtlinearität erforderlich, da die in Kap. 4 erarbeiteten 
Strategien nicht durchgehend den im Choice-Experiment angebotenen Ausprägungen entsprechen. Daher wurden für Attribute, deren Ausprägungen ein prozentuales Maß darstellen (Maisanteil an der Ackerfläche, Grünlandanteil an der landwirtschaftlich genutzten Fläche, Waldanteil an der Gesamtfläche), eine quadratische Nutzenfunktion unterstellt, sodass der Nutzen bei steigendem Flächenanteil des jeweiligen Attributes bis zu einem optimalen Wert steigt, und nach Erreichen dieses Schwellenwertes wieder abnimmt. Beispielsweise ist bei einem geringen Grünlandanteil eine Erhöhung nutzensteigernd, bei einem sehr hohen Grünlandanteil allerdings nutzenmindernd. Die übrigen zwei Attribute - Größe von Wald- und Feldstücken und Vogelindikator - wurden Dummy-kodiert. Dabei wurde der Status quo als Referenzsituation genommen, sodass die Werte mit positivem Vorzeichen den Eurobetrag darstellen, den der Befragte durchschnittlich bereit ist, für die Veränderung zu zahlen, ausgehend von seiner heutigen Situation. Beispielsweise wäre ein Befragter bereit, durchschnittlich $22 €$ zu zahlen, wenn der Vogelindikator in seinem Wohnumkreis auf 85 Punkte erhöht wird, und $53 €$ für eine Erhöhung auf 105 Punkte. Ein negatives Vorzeichen der Zahlungsbereitschaft entspricht dem Betrag, den der Befragte zahlen würde, um von einer hypothetischen Verschlechterung zurück in den Status quo zu wechseln. Beispielsweise liegt die Zahlungsbereitschaft für eine Verdopplung der derzeitigen Feldflächengrößen bei minus $30 €$. Würde man also die Größe der Wald- und Feldflächen verdoppeln, würde er $30 € \mathrm{zu}$ zahlen bereit sein, um wieder in seinen Ausgangszustand zu gelangen. Bei den Attributen „Waldanteil“", „Maisanteil““ und „Grünlandanteil“ können die marginalen Zahlungsbereitschaften bei deutlich höheren oder niedrigeren Status quo-Anteilen unterschiedlich sein. Die marginale Zahlungsbereitschaft für $10 \%$ mehr Waldanteil liegt bei einem Status quo-Waldanteil von unter $5 \%$ zwischen 90 und $100 €$, bei einem Waldanteil von $80 \%$ dagegen bei nahezu null.

Für eine Halbierung sowie für eine Verdopplung der durchschnittlichen Größe von Feldern und Waldstücken bestehen negative Zahlungsbereitschaften von 34 bzw. $30 €$. Im Durchschnitt möchten Befragte bei diesem Attribut lieber keine Veränderung des heutigen Zustandes.

Eine Erhöhung der Biodiversität, gemessen mit Hilfe des Vogelindikators bezogen auf landwirtschaftliche Flächen, wird als positiv wahrgenommen. Der aktuelle deutschlandweite Wert liegt bei 65 Punkten. Eine Erhöhung auf 85 Punkte ist den Befragten im Durchschnitt $22 €$ wert, eine Erhöhung auf 105 Punkte $53 €$. Die Zahlungsbereitschaft für eine Erhöhung des Maisanteils hängt vom Status quo ab. In unserem Sample haben die Befragten im Durchschnitt einen Maisanteil von $15 \%$. An dieser Stelle besteht eine geringe Zahlungsbereitschaft für eine Erhöhung des Maisanteils (Abb. 5.4). Bei ca. $20 \%$ liegt der optimale Maisanteil - hier liegt die Zahlungsbereitschaft für eine Erhöhung bei null. Bei Anteilen oberhalb des Optimums besteht eine Zahlungsbereitschaft für eine Verringerung. Je höher der Status quo-Maisanteil des Befragten, desto höher ist seine Zahlungsbereitschaft für eine 10-\%ige Verringerung. Bei $50 \%$ Maisanteil wird eine $10-\%$ ige Verringerung mit unter $20 €$ bewertet, bei $80 \%$ sind es knapp $40 €$. 

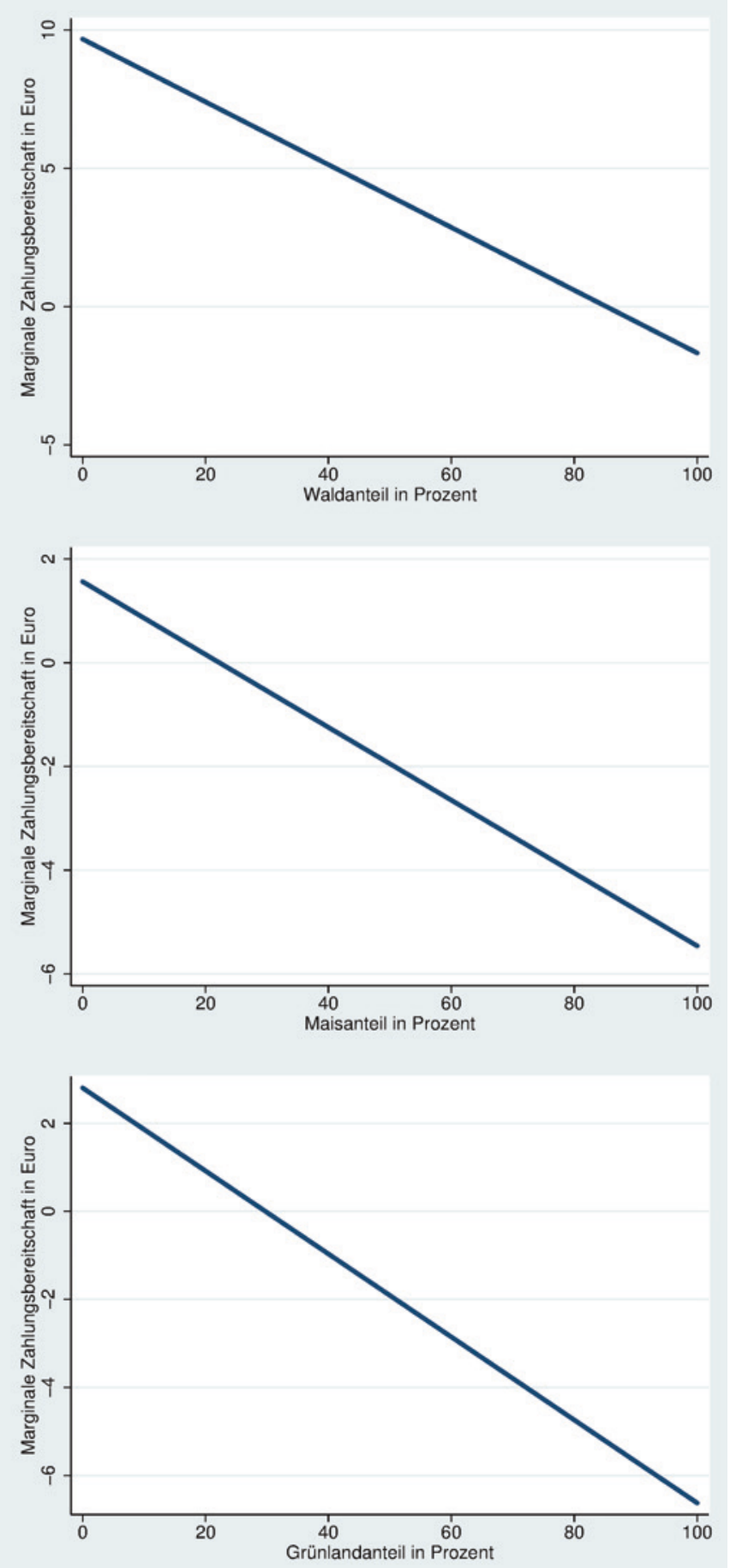

Abb. 5.4 Marginale Zahlungsbereitschaften in Abhängigkeit vom Status quo für Waldanteil (1), Maisanteil (2) und Grünlandanteil (3) 
Eine Erhöhung des Anteils von Wiesen und Weiden auf landwirtschaftlich genutzten Flächen wird überwiegend in Regionen mit geringem Grünlandanteil positiv bewertet. Der heutige Wert liegt in der Umgebung der Befragten bei $17 \%$ und damit deutlich unter dem bundesdeutschen Durchschnitt von von $28 \%$ (Statistisches Bundesamt). Eine Erhöhung des Grünlandanteils wird an dieser Stelle als positiv wahrgenommen, was sich in einer positiven Zahlungsbereitschaft von $14 €$ pro $10 \%$ mehr Grünland ausdrückt. Wie in Abb. 5.4 ersichtlich, ändert sich das Vorzeichen der marginalen Zahlungsbereitschaft jedoch bei ca. $22 \%$. Eine Erhöhung auf über $30 \%$ ist von den Befragten offenbar nicht gewollt. Tab. 5.2 gibt die durchschnittlichen Zahlungsbereitschaften für die Attribute wieder und Abb. 5.4 zeigt die marginale Zahlungsbereitschaft für Waldanteil, Maisanteil und Grünlandanteil in Abhängigkeit zum Status quo-Anteil grafisch.

Zusammengenommen deuten die Ergebnisse darauf hin, dass Landnutzungsänderungen - v. a. solche, die den Waldanteil und die Biodiversität erhöhen - zusätzlichen Nutzen in nicht zu vernachlässigender Höhe generieren könnten, während Landnutzungsänderungen, die den Waldanteil reduzieren und den Maisanteil erhöhen, Nutzeneinbußen verursachen, die den zusätzlichen landwirtschaftlichen Erträgen gegenüberstehen. In Abschn. 5.1.2.4 werden die Nutzen- und Kostenänderungen der kulturellen Ökosystemleistungen für die in Kap. 4 entwickelten Strategien berechnet und den Kosten- und Nutzenänderungen der bereitstellenden und regulierenden Ökosystemleistungen gegenübergestellt.

Tab. 5.2 Zahlungsbereitschaft (ZB) pro Kopf für landwirtschaftliche Merkmale, gemessen am durchschnittlichen Status quo in € (Fragebogenversionen 3 und 4). (Quelle: J. Sagebiel)

\begin{tabular}{l|l|l|c}
\hline Merkmalsausprägung & $\begin{array}{l}\text { Zahlungsbereitschaft in } \\
\text { € pro Kopf pro Jahr }\end{array}$ & $\mathbf{9 5} \%$ Konfidenzintervall \\
\cline { 3 - 4 } & Unteres Limit & Oberes Limit \\
\hline $\begin{array}{l}\text { Waldanteil an Gesamt- } \\
\text { fläche (10\% Erhöhung) }\end{array}$ & $\mathbf{7 5 * * *}$ & 66 & 93 \\
\hline Feldgrößen halbieren & $\mathbf{- 3 4 * * *}$ & -46 & -20 \\
\hline Feldgrößen verdoppeln & $\mathbf{- 3 0 * * *}$ & -46 & -17 \\
\hline $\begin{array}{l}\text { Vogelindikator für Bio- } \\
\text { diversität 85 Punkte }\end{array}$ & $\mathbf{2 2 * * *}$ & 11 & 33 \\
\hline $\begin{array}{l}\text { Vogelindikator für Bio- } \\
\text { diversität 105 Punkte }\end{array}$ & $\mathbf{5 3 * * *}$ & 40 & 67 \\
\hline $\begin{array}{l}\text { Maisanteil auf Acker- } \\
\text { fläche (10\% Erhöhung) }\end{array}$ & $\mathbf{5 , 1}$ & $-3,5$ & 14 \\
\hline $\begin{array}{l}\text { Grünlandanteil auf land- } \\
\text { wirtschaftlichen Flächen } \\
\text { (10\% Erhöhung) }\end{array}$ & $\mathbf{1 4 *}$ & $-1,7$ & 29 \\
\hline
\end{tabular}

*Signifikanzniveaus: $\mathrm{p}<0,05$; ** $\mathrm{p}<0,01$; *** $\mathrm{p}<0,001$ 


\subsubsection{Aggregierte Ergebnisse der ökonomischen Bewertung der Ökosystemleistungen der landwirtschaftlichen Landnutzungsstrategien}

Jesko Hirschfeld und Julian Sagebiel

Nachfolgend werden die Gesamtkosten und -nutzen der drei landwirtschaftlichen Strategien berechnet, die in Tab. 5.3 im Hinblick auf die Ausprägungen der jeweiligen Attribute für die landwirtschaftlichen Landnutzungen wiedergegeben sind. Eine analoge Berechnung der waldbezogenen Strategien findet sich in Abschn. 5.1.3.3.

Tab. 5.3 zeigt die aggregierten Bewertungsergebnisse in Bezug auf die untersuchten landwirtschaftlichen Strategien. Zur Bewertung der bereitstellenden Ökosystemleistungen wurden die Ergebnisse der agrarökonomischen Analysen aus Kap. 4, und zur Bewertung der regulierenden Ökosystemleistungen Ergebnisse aus Abschn. 5.1.2.2 genutzt. Zur Bewertung der kulturellen Ökosystemleistungen wurde die sogenannte „Kompensierende Variation“ berechnet. Das ist ein Wohlfahrtsmaß, welches für die jeweilige Strategie die notwendige monetäre Kompensation (z. B. als pauschal ausgezahlte Subvention oder erhobene Steuerzahlung) beschreibt, die notwendig ist, damit die Person weder besser noch schlechter gestellt wird als ohne die gewählte Strategie (Hanley und Barbier 2009, S. 16 ff.).

Hieraus ergibt sich für die auf größtmöglichen Klimaschutz orientierte Strategie im Bereich Landwirtschaft eine über die volljährige Bevölkerung in Deutschland aggregierte Zahlungsbereitschaft von $4 \mathrm{Mrd}$. €/Jahr. Dabei schlägt vor allem die hohe Wertschätzung einer Erhöhung des Waldanteils und die deutliche Reduzierung der durch die Landwirtschaft verursachten direkten und indirekten THG-Emissionen positiv zu Buche und vermag die betrieblichen Verluste durch Ertragseinbußen und Verringerung der landwirtschaftlich zu nutzenden Fläche mehr als zu kompensieren.

Die auf die Erzeugung von Biomasse zur energetischen Verwertung orientierte Strategie trifft auf eine geringere Zahlungsbereitschaft (0,4 Mrd. €). Auch hier kommt ein starker Klimaschutzeffekt zustande, doch die negativen Effekte durch höhere Nährstoffausträge ins Grundwasser und die Ablehnung von mehr Mais im Landschaftsbild durch die Bevölkerung ab einem Maisanteil von $20 \%$ führen zu einem insgesamt nur leicht positiven Gesamtnutzen dieser Strategie. Dies gilt allerdings nur unter der Annahme, dass durch eine weitere Zunahme des Maisanteils die Biodiversität auf den landwirtschaftlichen Flächen nicht noch weiter beeinträchtigt wird. Sollte der Vogelindikator sich durch eine Zunahme von Maisflächen dagegen weiter verschlechtern, würde die Strategie "Bioenergie“ in eine eindeutig negative Gesamtbewertung abrutschen.

Ebenfalls sehr positiv bewerteten die Bürger die umwelt- und naturschutzorientierte Strategie. Für die Umsetzung dieser Strategie besteht nach den Ergebnissen der Befragungsstudie eine Zahlungsbereitschaft von jährlich 2,7 Mrd. €. Diese Strategie punktet vor allem durch ihren positiven Effekt auf die Biodiversität auf Agrarflächen, was durch eine 10-\%ige Flächenstilllegung und eine Reduktion des Dünge- und 
Tab.5.3 Ökonomische Gesamtbewertung der Strategien „Klimaschutz“, „Bioenergie“ und „Umwelt- und Naturschutz“ für den Landwirtschaftssektor. (Quelle: J. Hirschfeld und J. Sagebiel)

\begin{tabular}{|c|c|c|c|c|}
\hline \multicolumn{2}{|l|}{ Strategien } & \multirow[t]{2}{*}{ Klimaschutz } & \multirow[t]{2}{*}{ Biomasse } & \multirow{2}{*}{$\begin{array}{l}\text { Umwelt- und } \\
\text { Naturschutz }\end{array}$} \\
\hline Effekte im Vergleich zur Referenzprojektion & Einheit & & & \\
\hline \multicolumn{5}{|l|}{ Bereitstellende Ökosystemleistungen } \\
\hline $\begin{array}{l}\text { Änderung der landwirtschaftlichen } \\
\text { Nettowertschöpfung zu Faktorkosten }\end{array}$ & Mio. $€$ & -1.715 & -141 & -1.393 \\
\hline \multicolumn{5}{|l|}{ Regulierende Ökosystemleistungen } \\
\hline Änderung Stickstoffges.-Bilanz & Mio. $t$ & $-0,051$ & $+0,041$ & $-0,051$ \\
\hline $\begin{array}{l}\text { Änderung Stickstoffges.-Austräge ins } \\
\text { Sickerwasser }\end{array}$ & Mio. $t$ & $-0,025$ & $+0,033$ & $-0,049$ \\
\hline $\begin{array}{l}\text { Geldwerter Nutzen der Veränderung der } \\
\text { Stickstoffausträge }\end{array}$ & Mio. $€$ & $\begin{array}{c}160 \\
76 ; 509\end{array}$ & $\begin{array}{c}-206 \\
-98 ;-653\end{array}$ & $\begin{array}{c}306 \\
146 ; 971\end{array}$ \\
\hline $\begin{array}{l}\text { Änderung der THG-Emissionen ohne indirekte } \\
\text { Landnutzungsänderungen (iLUC) in Mio. } \mathrm{CO}_{2-} \\
\text { Äqu. }\end{array}$ & Mio. $t$ & $-15,3$ & $-18,1$ & $-10,1$ \\
\hline $\begin{array}{l}\text { Änderung THG-Emissionen durch indirekte } \\
\text { Landnutzungsänderungen in Mio. t } \mathrm{CO}_{2} \text {-Äqu. }\end{array}$ & Mio. $t$ & $+3,5$ & $+3,6$ & $+4,2$ \\
\hline $\begin{array}{l}\text { Gesamte Änderung der THG-Emissionen (inkl. } \\
\text { iLUC) in Mio. t } \mathrm{CO}_{2} \text {-Äqu. }\end{array}$ & Mio. $t$ & $-11,8$ & $-14,5$ & $-5,8$ \\
\hline $\begin{array}{l}\text { Geldwerter Nutzen der Verringerung der THG- } \\
\text { Emissionen }\end{array}$ & Mio. $€$ & $\begin{array}{c}944 \\
94 ; 1.416\end{array}$ & $\begin{array}{c}\mathbf{1 . 1 6 0} \\
116 ; 1.740\end{array}$ & $\begin{array}{c}464 \\
46 ; 696\end{array}$ \\
\hline \multicolumn{5}{|l|}{ kulturelle Ökosystemleistungen } \\
\hline Landschaftsbild Waldanteil & $\%$ & $+10 \%$ & konstant & konstant \\
\hline Zahlungsbereitschaft Landschaftsbild (Wald) & Mio. $€$ & $\begin{array}{c}4.635 \\
4.079 ; 5.747\end{array}$ & 0 & 0 \\
\hline Biodiversität (Vogelindikator) & Pkte. & konstant & konstant & $\begin{array}{l}\text { deutlich } \\
\text { erhöht }\end{array}$ \\
\hline $\begin{array}{l}\text { Zahlungsbereitschaft Biodiversität } \\
\text { (Vogelindikator) }\end{array}$ & Mio. $€$ & 0 & 0 & $\begin{array}{c}3.275 \\
2.472 ; 4.141\end{array}$ \\
\hline Maisanteil bis zu x \% der LF & $\%$ & konstant & $30 \%$ & konstant \\
\hline Zahlungsbereitschaft Landschaftsbild (Mais) & Mio. $€$ & 0 & $\begin{array}{c}-375 \\
-1.121 ; 372\end{array}$ & 0 \\
\hline Ökonomischer Gesamtnutzen pro Jahr & Mio. $€$ & $\begin{array}{c}4.024 \\
2.535 ; 5.958\end{array}$ & $\begin{array}{c}439 \\
-1.244 ; 1.319\end{array}$ & $\begin{array}{c}2.652 \\
1.271 ; 4.415\end{array}$ \\
\hline \multicolumn{5}{|c|}{$\begin{array}{l}\text { Die Effekte der Strategien auf bereitstellende, regulierende und kulturelle Ökosystemleistungen } \\
\text { und ihre ökonomische Bewertung, aggregiert über die volljährige Bevölkerung in Deutschland } \\
\text { (monetäre Werte grau unterlegt, Spannbreite der monetären Bewertungen jeweils zweite Zeile der } \\
\text { Bewertungszellen: Untergrenze; Obergrenze). } \\
\text { Eine analoge Berechnung der forstwirtschaftlichen Strategien findet sich in Abschn. 5.1.3.3 }\end{array}$} \\
\hline
\end{tabular}


Pflanzenschutzmitteleinsatzes erreicht werden kann. Sehr positiv wird der deutliche Beitrag zur Verringerung der Nitrateinträge ins Grundwasser bewertet. Die Emissionen von Treibhausgasen werden nach den hier getroffenen Annahmen nicht so stark vermindert wie in den beiden anderen Strategien - dies vor allem, weil durch die Flächenstilllegungen und Intensitätsverringerung auf deutschen Agrarflächen bei gleichbleibendem Konsum die Importe von Nahrungsmitteln aus dem Ausland dort Landnutzungsänderungen anstoßen könnten, die dort $\mathrm{zu}$ mehr THG-Emissionen führen würden. Gleichwohl kommt trotz dieser Gegenrechnung noch ein deutlicher Klimaschutzeffekt zustande: 5,8 Mio. t $\mathrm{CO}_{2}$-Äqu. können durch diese Strategie vermieden werden. ${ }^{4}$ Würde diese Strategie um eine Aufforstungskomponente ergänzt, könnte ein noch höherer gesamtgesellschaftlicher Nutzen erzielt werden (bis zu etwa 6 Mrd. € jährlich).

Zur Abbildung der Streuung der monetären Bewertungsergebnisse aus der Perspektive unterschiedlicher, extrem geringer oder extrem hoher Wertschätzungen werden in den grauen Zeilen, in denen die monetären Bewertungsergebnisse wiedergegeben werden, jeweils in der zweiten Zeile die Unter- und Obergrenzen der jeweiligen Bewertungen angegeben. Im Fall der Bewertung der Nährstoffausträge ins Grundwasser wird die Untergrenze von $3 € / \mathrm{kg} \mathrm{N}$ und eine Obergrenze von $20 € / \mathrm{kg} \mathrm{N}$, bezüglich der Vermeidung von THG-Emissionen eine Spanne von $8 € / t \mathrm{CO}_{2}$ - ̈̈qu. bis $120 € / \mathrm{t}$ $\mathrm{CO}_{2}$ - ̈̈qu. angesetzt (vgl. dazu Abschn. 5.1.2.2). Bei den kulturellen Ökosystemleisten werden dafür aus der Zahlungsbereitschaftsstudie die Ober- und Untergrenzen des $95 \%$-Konfidenzintervalls genutzt, d. h., nur $5 \%$ der Befragten haben eine noch niedrigere oder noch höhere Zahlungsbereitschaft als die Befragten innerhalb dieses Intervalls.

Diese Sensitivitätsanalyse der Gesamtbewertung zeigt die klimaschutzfokussierte sowie die natur- und umweltschutzorientierte Strategie sehr robust im deutlich positiven Bereich, während die Bioenergiestrategie im Fall extremerer Bewertungen sowohl auf eine positive als auch auf eine negative Gesamtbewertung kommen kann. Im Falle einer (hier nicht berücksichtigten) negativen Auswirkung auf die Biodiversität, würde die Bioenergiestrategie in den klar negativen Bereich fallen.

Um die Transparenz der Bewertung zu erhöhen, gibt Tab. 5.3 nicht nur die monetarisierten Werte wieder (in den grau unterlegten Zeilen), sondern auch die physischen Ausgangswerte, auf die die monetären Bewertungen Bezug nehmen. Damit bleibt es den Rezipienten dieser Untersuchung offen, ob sie sich den durch die Autoren ermittelten Spannbreiten der gesellschaftlichen Wertschätzung (übersetzt in monetäre Werte) anschließen möchten - oder aber die verschiedenen Kriterien lieber einer davon unabhängigen eigenen multikriteriellen Bewertung unterziehen wollen.

\footnotetext{
${ }^{4}$ Im Inland könnten durch eine Umsetzung der Natur- und Umweltschutzstrategie 10,1 Mio. $\mathrm{CO}_{2^{-}}$ Äqu. vermieden werden.
} 


\subsection{3 Ökonomische Bewertung der kulturellen Ökosystemleistungen des Waldes}

Priska Weller und Peter Elsasser

\subsubsection{Ergebnisse zu Naturschutz- und Landschaftspflegeleistungen im Wald}

Priska Weller und Peter Elsasser

Zur Bewertung der forstlichen Landnutzung wurden Maßnahmenbündel identifiziert, die die Strategien „Klimaschutz“, „Bioenergie“, „Natur- und Umweltschutz“ und „Klimaanpassung" sowie die Referenzprojektion konkretisieren (vgl. Abschn. 4.6.1 und 4.6.2). Diese Maßnahmenbündel bestehen aus Einzelmaßnahmen, welche dann, je nach Zielsetzung in unterschiedlicher Ausprägung, im Wald umgesetzt werden. Die Maßnahmen wiederum haben neben den Klimawirkungen und betrieblichen Wirkungen auch Wirkungen auf die kulturellen Ökosystemleistungen des Waldes. Diese sollen mittels Präferenzoffenbarungsmethoden, insbesondere Choice-Experimenten, bewertet werden. Der Fokus liegt auf den kulturellen Ökosystemleistungen des Waldes, insbesondere Naturschutz (Artenvielfalt) und Landschaftsqualität. Dafür werden speziell die in Abschn. 5.1.1.3 erläuterten acht Merkmale des Themenschwerpunkts „Wald“ herangezogen.

\section{Merkmale im Themenschwerpunkt ,Wald“6}

Die Merkmale des Waldes wurden so gewählt, dass sie Naturschutz- und Landschaftspflegeleistungen des Waldes abbilden. Zur Bewertung der Naturschutzleistung des Waldes (d.h. der Beitrag des Waldes zum Naturschutz am Wohnort der Befragten) werden die Artenvielfalt im Wald und der Anteil ungenutzter Waldflächen herangezogen. Zur Bewertung der Artenvielfalt wurde (analog zum Themenfeld „Landwirtschaft") ein Indikator mit einer Punkteskala verwendet (vgl. Tab. 5.1) (Artenvielfalt: wie heute; 85 Punkte; 105 Punkte). Dieser Indikator wird auch in der Nachhaltigkeitsstrategie der Bundesregierung (2002) verwendet und misst über die Zählung relevanter Vogelarten die Artenvielfalt in sechs verschiedenen Landschaftstypen, da diese Vogelarten gleichzeitig Auskunft über das Vorhandensein anderer Tier- und Pflanzenarten geben (Bundesregierung 2002). Außerdem wird das Merkmal „Anteil ungenutzter Waldflächen“ zur Bewertung der Naturschutzleistung herangezogen. Dies ist der aktuellen politischen Diskussion geschuldet ${ }^{5}$ sowie der Annahme, dass biologische Prozesse in ungenutzten Wäldern natürlicher ablaufen und dies zu einer naturnäheren Entwicklung führt.

Die Landschaftspflegeleistung des Waldes (d. h. der Einfluss des Waldes auf das Landschaftsbild am Wohnort der Befragten) wird durch die Merkmale „Anteil Wald an

\footnotetext{
${ }^{5}$ Nach der Nationalen Biodiversitätsstrategie sollen $5 \%$ der deutschen Wälder bis 2020 der ,natürlichen Waldentwicklung“ überlassen und dauerhaft aus der Nutzung genommen werden (BMU 2007).
} 
der Landschaft" sowie „Größe einzelner Felder und Waldstücke“ beschrieben. Weiterhin ist der innere Aufbau des Waldes nutzenrelevant für die Bevölkerung. Dies können mehr oder weniger Waldflächen mit Unterwuchs durch junge Bäume und Sträucher sein wie auch das Alter der Wälder. Des Weiteren kann die Baumartenwahl über den Nadelbaumanteil und den Anteil von Bäumen aus anderen Ländern den Landschaftseindruck stark beeinflussen. Sowohl die Merkmale zum „Äußeren“ des Waldes als auch die zum „Inneren“ des Waldes werden zur Bewertung herangezogen.

\section{Allgemeine Ergebnisse der Befragung}

Zusätzlich zu den soziodemographischen Angaben sind in der Befragung Angaben zu Einstellungen und Aktivitäten in der Wohnumgebung erhoben worden. Dabei stellte sich heraus, dass ca. $60 \%$ der Befragten im Schnitt mindestens einmal pro Woche ,in ihrer Freizeit in der freien Landschaft" sind (d. h., Arbeitswege o. Ä. sind hierin nicht enthalten). Die Meisten halten sich dabei in einer Landschaft mit Wald oder mit Wald und Offenland auf, auch Gewässer (Seen, Flüsse) sind beliebt. Dabei finden die Meisten (70 \%), dass der Zustand der Landschaft gleich geblieben ist, seit sie dort leben. Jeweils etwa $15 \%$ bemerkten eine Verbesserung oder Verschlechterung.

Im Anschluss an das Choice-Experiment wurde die individuelle Bedeutung der einzelnen Merkmale für die Entscheidung der Befragten mittels fünfstufiger Skala erfragt. Dabei gaben $90 \%$ der Befragten an, der Anteil von Wald an der Landschaft sei ihnen sehr wichtig oder wichtig. $83 \%$ der Befragten finden Artenvielfalt im Wald sehr wichtig oder wichtig. Demgegenüber finden nur $23 \%$ der Befragten den Anteil von Bäumen aus anderen Ländern sehr wichtig oder wichtig. Insgesamt kann man aber aus den Antworten schließen, dass die Fragen und Merkmale im Fragebogen von Relevanz für die Bevölkerung sind.

Zur besseren Einordnung der Befragungsergebnisse wurden die Befragten gebeten, den aktuellen Zustand der Landschaft in ihrer Wohnumgebung in Bezug auf die untersuchten Merkmale einzuschätzen und anhand einer fünfstufigen Skala zu bewerten. Die unterschiedlichen Wahrnehmungen bzw. die durch den Befrager bereitgestellten Ausgangssituationen haben Auswirkungen auf die Befragungsergebnisse, namentlich auch die Zahlungsbereitschaften (siehe dazu Marsh et al. 2011 oder Dominguez-Torreiro und Solino 2011). Da der wahrgenommene Status quo die Grundlage für die Bewertung im Choice-Experiment und die resultierenden Zahlungsbereitschaften ist, ist es wichtig, diese Größen zu betrachten. Dies geschieht unter der Annahme, dass die Befragten ihre Wohnumgebung realistisch einschätzen können. Es wird deutlich, dass die Mehrheit der Befragten zu einer „mittleren“ Einschätzung der meisten Merkmale tendiert, gleichwohl geben $40 \%$ der Befragten an, nach ihrer persönlichen Einschätzung einen sehr hohen oder hohen Waldanteil in ihrer Wohnumgebung zu haben. Weiterhin bemerken $33 \%$ eine sehr hohe oder hohe Artenvielfalt, und $55 \%$ der Befragten geben an, dass nur ein niedriger oder sehr niedriger Anteil der Waldflächen in ihrer Umgebung ungenutzt ist. Der Anteil von Bäumen aus anderen Ländern wird von $83 \%$ der Befragten als niedrig oder sehr niedrig angegeben. 
Zusätzlich zu den soziodemographischen Angaben ist bekannt, dass etwa $4 \%$ der Befragten Wald besitzen. Über die Hälfte dieser Personen bewirtschaften ihren Wald selbst. Etwa zwei Drittel besitzen zusätzlich zum Wald noch landwirtschaftliche Flächen. $5 \%$ der Befragten arbeiten in einem Beruf mit Bezug zu Landnutzung (Landwirtschaft, Forstwirtschaft, Naturschutz/Umweltschutz, Landschaftsplanung, Tourismus) und $12 \%$ der Befragten sind Mitglied in einer Natur- oder Umweltschutzorganisation. Abb. 5.5 zeigt die auf freiwilliger Basis angegebenen Wohnorte der Befragten im Themenschwerpunkt „Wald“, dabei wird zwischen Stichprobe 1 und Stichprobe 2 unterschieden.

\section{Bewertungsergebnisse}

Die Modelle werden auf Grundlage des Datensatzes bzw. der beiden Stichproben berechnet und geben Durchschnittswerte über alle Befragten an. Da die Stichproben repräsentativ ausgewählt wurden, gelten die Ergebnisse durchschnittlich für die deutsche

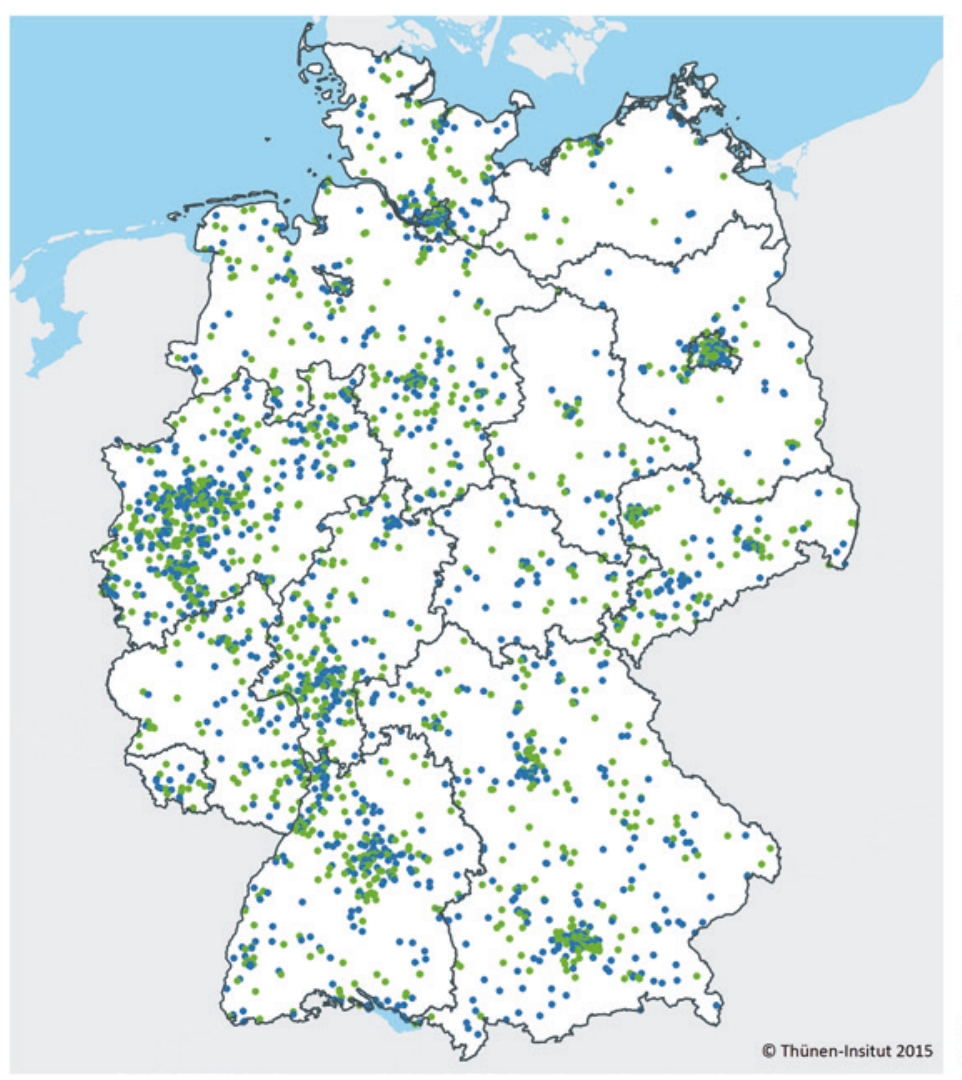

Quelle: ATKIS VG1000, Bundesamt für Kartographie und Geodãsie 2014

Abb. 5.5 Geografische Verortung der Befragten des Samples „Wald“ 
Bevölkerung. Für die Merkmale kann nicht automatisch ein linearer Zusammenhang zwischen der Erhöhung eines Merkmals und den Nutzenzuwachs angenommen werden, deshalb wurden die Merkmale als Dummy-Variablen in das Modell integriert. Des Weiteren sehen die zu bewertenden Strategien $10 \%$ mehr Waldanteil an der Landschaft und $10 \%$ ungenutzte Waldflächen vor, und die Ergebnisse der entsprechenden DummySchätzungen können direkt in die Bewertung der Strategien einfließen.

Das verwendete „Conditional logit“-Modell zeigt signifikante Ergebnisse für die meisten Variablen in beiden Wald-Stichproben (vgl. Tab. 5.4). Der Preiskoeffizient ist in beiden Stichproben negativ, was eine sinkende Auswahlwahrscheinlichkeit mit steigendem Preis indiziert. Die alternativenspezifische Konstante ist nicht signifikant, was darauf hinweist, dass die Befragten keine ausdrückliche Präferenz für die Beibehaltung des aktuellen Status quo in ihrer Wohnumgebung haben. Dies schließt aber nicht aus, dass es Präferenzen für die Beibehaltung einzelner Aspekte des Status quo gibt. In beiden Stichproben wird die Verkleinerung des Waldanteils in der Landschaft negativ bewertet, eine Vergrößerung des Waldanteils um $10 \%$ positiv bewertet. Die jeweiligen Werte der Koeffizienten liegen nah beieinander. Bei der Veränderung der Größe einzelner Felder und Waldstücke werden in beiden Stichproben sowohl Vergrößerungen als auch Verkleinerungen negativ bewertet. Dies kann man als Zufriedenheit der Befragten mit der Landschaft in ihrer Wohnumgebung interpretieren.

In Stichprobe 1 hat eine mögliche Halbierung der Menge an Unterwuchs, jungen Bäumen und Sträuchern einen negativen Einfluss auf die Auswahlwahrscheinlichkeit der entsprechenden Landschaftsoption, während eine Verdopplung kein signifikantes Ergebnis zeigt. Den Anteil von Nadelbäumen auf $70 \%$ zu verändern ${ }^{6}$ hat einen negativen Einfluss auf den Nutzen der Befragten, während eine Veränderung auf $30 \%$ nicht signifikant positiv oder negativ bewertet wurde. Hierbei ist der Status quo zu berücksichtigen, von dem aus die Veränderung jeweils vorgenommen wird, da dies dann entsprechend eine Erhöhung oder Reduktion bedeuten kann. Das Erntealter von Wäldern im Durchschnitt um 20 Jahre zu erhöhen, hat einen positiven Einfluss auf die Zahlungsbereitschaft, während die Erhöhung im Durchschnitt um 30 Jahre keinen signifikanten Einfluss hat.

In Stichprobe 2 werden sowohl eine leichte als auch eine deutliche Erhöhung der Artenvielfalt in deutschen Wäldern positiv bewertet. Eine deutliche Erhöhung wird dabei (wie erwartet) stärker positiv bewertet als eine leichte Erhöhung. Den Anteil ungenutzter Waldflächen auf $0 \%$ zu verändern (d. h. zu reduzieren) und folglich die gesamte Waldfläche zu nutzen, wird von den Befragten negativ bewertet. Der Koeffizient für $10 \%$ ungenutzte Waldflächen ist nicht signifikant. Den Anteil von Bäumen aus anderen Ländern zu halbieren sowie zu verdoppeln wird von den Befragten jeweils negativ bewertet. Sowohl eine starke Ausweitung als auch der komplette Verzicht auf den Anbau

\footnotetext{
${ }^{6}$ Angesichts der derzeitigen Verteilung von Laub- und Nadelbäumen bedeutet dies i. d. R. eine Erhöhung des Nadelbaumanteils (BMEL 2015).
} 
Tab. 5.4 Koeffizienten aus dem Modell für einzelne Waldmerkmale (Fragebogenversionen 1 und 2). (Quelle: P. Weller)

\begin{tabular}{|c|c|c|}
\hline Stichprobe 1 & Koeffizient & Teststatistik (z) \\
\hline Alternativenspez. Konstante & 0,0717 & 0,97 \\
\hline Anteil Wald $(-10 \%)$ & $-0,4529$ & $-9,19$ \\
\hline Anteil Wald (+10\%) & $\mathbf{0 , 3 5 7 1}$ & 9,47 \\
\hline Feldgröße (Halbierung) & $-0,2555$ & $-5,86$ \\
\hline Feldgröße (Verdopplung) & $-0,2310$ & $-6,10$ \\
\hline Unterwuchs (Halbierung) & $-0,3872$ & $-8,17$ \\
\hline Unterwuchs (Verdopplung) & $-0,0300$ & $-0,72$ \\
\hline Nadelbaumanteil (30 \%) & $-0,0859$ & $-1,88$ \\
\hline Nadelbaumanteil (70 \%) & $-0,7020$ & $-15,23$ \\
\hline Erntealter (+20 Jahre) & 0,2129 & 4,18 \\
\hline Erntealter (+30 Jahre) & 0,0226 & 0,49 \\
\hline Preis & $-0,0056$ & $-13,24$ \\
\hline \multicolumn{3}{|l|}{ Stichprobe 2} \\
\hline Alternativenspez. Konstante & 0,0941 & 1,28 \\
\hline Anteil Wald $(-10 \%)$ & $-0,4354$ & $-8,49$ \\
\hline Anteil Wald (+10 \%) & 0,3115 & 8,26 \\
\hline Feldgröße (Halbierung) & $-0,2550$ & $-5,84$ \\
\hline Feldgröße (Verdopplung) & $-0,1805$ & $-4,78$ \\
\hline Artenvielfalt (leicht erhöhen auf 85 P.) & 0,2601 & 5,60 \\
\hline Artenvielfalt (stark erhöhen auf 105 P.) & 0,2967 & 7,28 \\
\hline Ungenutzte Waldflächen $(0 \%)$ & $-0,2798$ & $-6,01$ \\
\hline Ungenutzte Waldflächen (10 \%) & $-0,0225$ & $-0,52$ \\
\hline Bäume aus anderen Ländern (Halbierung) & $-\mathbf{0 , 1 5 9 0}$ & $-3,38$ \\
\hline Bäume aus anderen Ländern (Verdopplung) & $-0,8631$ & $-18,15$ \\
\hline Preis & $-0,0047$ & $-11,46$ \\
\hline
\end{tabular}

Hinweis: Statistische Signifikanz (abhängig von z-Wert) wird durch Fettdruck angezeigt

solcher Baumarten wäre also nutzenmindernd. Insgesamt sind die Ergebnisse nicht immer linear über die Merkmalsausprägungen verteilt.

Das Netto-Haushaltseinkommen der Befragten wurde testweise ebenfalls in die Liste der Erklärungsvariablen bei der Modellberechnung aufgenommen und somit der Einfluss auf die Präferenzen getestet. Entgegen der ökonomischen Erwartungen war die 
Einkommensvariable allerdings in beiden Stichproben nicht signifikant, d. h., der Einfluss des Einkommens auf die Präferenzen der Bevölkerung war nicht eindeutig identifizierbar. Sie wird daher in den weiteren Modellanalysen nicht mehr berücksichtigt.

\section{Zahlungsbereitschaften pro Person für einzelne Waldmerkmale}

Die Zahlungsbereitschaftsanalyse betrachtet die gewählten Waldmerkmale im Abwägungsverhältnis gegen Geld. Dabei bezieht sie sich auf Euro, die eine Person pro Jahr zu bezahlen bereit ist. Die errechneten Zahlungsbereitschaften lassen sich für die Betrachtung von Landnutzungsstrategien oder Maßnahmenbündeln aggregieren.

Die Zahlungsbereitschaft der Befragten für Veränderungen einzelner Merkmale des Waldes ergibt sich aus dem Quotienten:

$$
\text { - Koeffizient } \text { Merkmal/Koeffizient }_{\text {Preis }}
$$

Daher ändern sich die oben dargestellten Signifikanzen und Muster in der daraus abgeleiteten Zahlungsbereitschaftsanalyse nicht (vgl. Tab. 5.5). Die negative Zahlungsbereitschaft, also die Entschädigungsforderung für eine Verringerung des Waldanteils um $10 \%$, liegt in den beiden Stichproben bei 80 bzw. $92 € / p / a$ (Person und Jahr), während eine Vergrößerung des Waldanteils um $10 \%$ eine positive Zahlungsbereitschaft von jeweils ca. 65 €/p/a generiert. ${ }^{7}$ Halbierung wie auch Verdopplung der Größe von Feldern und Waldstücken generiert Entschädigungsforderungen zwischen 40 und $55 € / \mathrm{p} / \mathrm{a}$. Die Reduktion von Unterwuchs im Wald führt ebenso zu einer Entschädigungsforderung wie ein Nadelbaumanteil von 70 \% (69 bzw. 125 €/p/a). Die Erhöhung des Erntealters um 20 Jahre generiert eine Zahlungsbereitschaft von 38 €/p/a. Die Erhöhung der Artenvielfalt im Wald ist der Bevölkerung 55 bis $63 € /$ p/a wert, je nach Ausmaß der Erhöhung. Die Entschädigungsforderung für $0 \%$ ungenutzte Waldflächen, also Nutzung aller zur Verfügung stehenden Waldflächen, beträgt etwa $60 € / p / a$, während eine Halbierung des Anteils von Bäumen aus anderen Ländern mit 34 €/p/a und eine Verdopplung des Anteils von Bäumen aus anderen Ländern sogar mit $180 € /$ p/a abgegolten werden müssten.

Diese Werte geben Größenordnungen an, deren Relationen zueinander von besonderem Interesse sind. Beispielsweise ist der Waldanteil eins der höchstbewerteten Merkmale, da seine Veränderung mit die höchsten positiven und negativen Zahlungsbereitschaften generiert. Insgesamt wird sichtbar, dass die Bevölkerung die meisten Änderungen an Merkmalen der Landschaft oder des Waldes mit negativem Nutzen bewertet. Lediglich Vergrößerungen des Waldanteils und der Artenvielfalt stiften durchgehend positiven Nutzen. Darüber hinaus unterliegen manche der Bewertungsergebnisse

\footnotetext{
${ }^{7}$ Diese Schätzungen entsprechen weitgehend der unabhängigen Schätzung aus dem landwirtschaftlichen Untersuchungsteil, obwohl diese auf anderen Stichproben basieren und mit einer anderen Spezifikation des Regressionsmodells berechnet wurden; insbesondere liegt die dortige Mittelwertschätzung (noch) in den hier angegebenen Konfidenzbereichen.
} 
Tab. 5.5 Zahlungsbereitschaften für einzelne Waldmerkmale ( $€ / \mathrm{p} / \mathrm{a})$ (Fragebogenversionen 1 und 2). (Quelle: P. Weller)

\begin{tabular}{|c|c|c|c|}
\hline Stichprobe 1 & $\begin{array}{l}\text { Zahlungsbereitschaft } \\
\text { (ZB) }\end{array}$ & $\begin{array}{l}\text { Untere Konfidenz- } \\
\text { grenze der ZB }\end{array}$ & $\begin{array}{l}\text { Obere Konfidenz- } \\
\text { grenze der ZB }\end{array}$ \\
\hline Anteil Wald (-10\%) & $-80,32$ & $-100,96$ & $-59,67$ \\
\hline Anteil Wald (+10 \%) & 63,33 & 49,67 & 77,00 \\
\hline $\begin{array}{l}\text { Feldgröße } \\
\text { (Halbierung) }\end{array}$ & $-45,31$ & $-62,85$ & $-27,76$ \\
\hline $\begin{array}{l}\text { Feldgröße (Ver- } \\
\text { dopplung) }\end{array}$ & $-40,96$ & $-57,03$ & $-24,89$ \\
\hline $\begin{array}{l}\text { Unterwuchs } \\
\text { (Halbierung) }\end{array}$ & $-68,67$ & $-90,35$ & $-46,99$ \\
\hline $\begin{array}{l}\text { Unterwuchs (Ver- } \\
\text { dopplung) }\end{array}$ & $-5,33$ & $-20,31$ & 9,66 \\
\hline $\begin{array}{l}\text { Nadelbaumanteil } \\
(30 \%)\end{array}$ & $-15,23$ & $-32,19$ & 1,73 \\
\hline $\begin{array}{l}\text { Nadelbaumanteil } \\
(70 \%)\end{array}$ & $-124,48$ & $-153,81$ & $-95,15$ \\
\hline Erntealter (+20 Jahre) & 37,75 & 20,51 & 54,98 \\
\hline Erntealter (+30 Jahre) & 4,01 & $-11,63$ & 19,64 \\
\hline \multicolumn{4}{|l|}{ Stichprobe 2} \\
\hline Anteil Wald $(-10 \%)$ & $-91,99$ & $-117,80$ & $-66,18$ \\
\hline Anteil Wald (+10 \%) & 65,82 & 49,40 & 82,23 \\
\hline $\begin{array}{l}\text { Feldgröße } \\
\text { (Halbierung) }\end{array}$ & $-\mathbf{5 3 , 8 7}$ & $-75,80$ & $-31,94$ \\
\hline $\begin{array}{l}\text { Feldgröße (Ver- } \\
\text { dopplung) }\end{array}$ & $-38,13$ & $-56,63$ & $-19,63$ \\
\hline $\begin{array}{l}\text { Artenvielfalt (leicht } \\
\text { erhöhen auf } 85 \text { P.) }\end{array}$ & 54,96 & 35,93 & 73,98 \\
\hline $\begin{array}{l}\text { Artenvielfalt (stark } \\
\text { erhöhen auf } 105 \text { P.) }\end{array}$ & 62,68 & 48,33 & 77,04 \\
\hline $\begin{array}{l}\text { Ungenutzte Wald- } \\
\text { flächen }(0 \%)\end{array}$ & $-59,11$ & $-84,30$ & $-33,92$ \\
\hline $\begin{array}{l}\text { Ungenutzte Wald- } \\
\text { flächen }(10 \%)\end{array}$ & $-4,75$ & $-23,07$ & 13,57 \\
\hline $\begin{array}{l}\text { Bäume aus anderen } \\
\text { Ländern (Halbierung) }\end{array}$ & $-\mathbf{3 3 , 5 8}$ & $-55,27$ & $-11,90$ \\
\hline $\begin{array}{l}\text { Bäume aus anderen } \\
\text { Ländern (Verdopplung) }\end{array}$ & $-182,35$ & $-226,41$ & $-138,28$ \\
\hline
\end{tabular}

Hinweis: Statistische Signifikanz wird durch Fettdruck angezeigt 
Unsicherheiten aufseiten der Bevölkerung und sollten mit Vorsicht interpretiert werden. Insbesondere bei den Merkmalen „Anteil von Bäumen aus anderen Ländern“ sowie „,ungenutzte Waldflächen“ geben die Befragten an, dass sie den aktuellen Zustand der einzelnen Merkmale nicht kennen und es bevorzugen, keine Einschätzung darüber abzugeben (jeweils 21 und $8 \%$ der Befragten). Dies könnte eine Erklärung dafür sein, dass eine Verdopplung des Anteils von Bäumen aus anderen Ländern bei jedem Ausgangsanteil abgelehnt wird, auch bei einem aktuell niedrigen Anteil. Veränderungen werden insgesamt aber abgelehnt, einschließlich einer Halbierung des Anteils von Bäumen aus anderen Ländern. Bezüglich der Ablehnung der $10 \%$ ungenutzten Waldflächen liegt die Interpretation nahe, dass dieser Wert von der Bevölkerung als Reduktion eingeschätzt und deshalb abgelehnt (n. s.) und negativ bewertet wurde. Aus anderen Studien ist bekannt, dass Befragte oft eher weniger Nutzung bevorzugen (z. B. Elsasser und Weller 2013), dazu passt auch die Ablehnung der $0 \%$ ungenutzten Waldflächen.

Die Befragungsergebnisse können im nächsten Schritt zu Strategien zusammengefasst werden (Abschn. 5.1.3.3) und auf die Bevölkerung hochgerechnet werden. Als Basis dienen die Eingangsdaten und Simulationsergebnisse aus Abschn. 4.6. Wie dort beschrieben, basieren diese Daten auf durchschnittlichen Verhältnissen in den Bundesländern. Von dieser Datenbasis ausgehend, wurde jeweils auf Bundesebene aggregiert. In einzelnen Fällen sind dazu zusätzliche Annahmen nötig, auf die an entsprechender Stelle hingewiesen wird.

\subsubsection{Einordnung der Ergebnisse und weiterer Leistungen des Waldes}

Priska Weller und Peter Elsasser

\section{Naturschutz- und Landschaftspflegeleistungen}

In der Vergangenheit hat es einige Studien zu Leistungen der Landschaft und insbesondere des Waldes gegeben, in denen Präferenzoffenbarungsmethoden verwendet wurden, und die nun zur Einordnung der beschriebenen Ergebnisse herangezogen werden sollen. Da der Fokus der Befragung auf der Bewertung von Naturschutz- und Landschaftspflegeleistungen lag, wird hier zunächst auf einige exemplarische Studien zu diesen beiden Themen eingegangen.

Für die Bewertung von Naturschutzmaßnahmen in Deutschland ist beispielsweise die Studie von Meyerhoff et al. (2012) wichtig. Meyerhoff et al. bewerten die Umsetzung der Nationalen Biodiversitätsstrategie in verschiedenen Ökosystemen und finden positive Ergebnisse, insbesondere für die den Wald betreffenden Maßnahmen. Sie ermitteln eine aggregierte Zahlungsbereitschaft (für 40,1 Mio. zahlungswillige Haushalte) in Höhe von 2,22 Mrd. €/Jahr für ein Wald-Maßnahmenbündel. Dieses besteht aus dem Zulassen natürlicher Waldentwicklung, Umbau in naturnahe Laub- und Mischbestände, Erhöhung der Strukturvielfalt durch Totholzanreicherung, Biotopbäume und Waldränder sowie dem Schutz bestehender Nieder- und Mittelwälder. Zusätzliche Vorsorgemaßnahmen zur Anpassung an Klimaänderungen erhöhten die Zahlungsbereitschaft nicht signifikant. 
Die Vorliebe für Naturnähe und die resultierende Präferenz für ökologisch unberührten Wald geht so weit, dass in einer Bevölkerungsbefragung von Müller und Job (2009) die Befragten dem Borkenkäfer im Nationalpark Bayerischer Wald gegenüber mehrheitlich neutral bis schützend (keine Bekämpfung gewünscht) eingestellt sind. Dies deckt sich mit den hier vorliegenden Befragungsergebnissen, nach denen die Bevölkerung insgesamt sehr naturschutzorientiert eingestellt ist und dies in den entsprechenden Zahlungsbereitschaften deutlich gemacht hat: Eine Erhöhung der Artenvielfalt wurde durchweg positiv bewertet, und die ungenutzten Waldflächen auf $0 \%$ der verfügbaren Fläche zu reduzieren wurde negativ bewertet, d. h., aus Sicht der Bevölkerung sollten ungenutzte Waldflächen erhalten bleiben.

Bei einer Bewertung des Landschaftsbildes im Nordosten Deutschlands haben Elsasser et al. (2010) mittels Befragung eine Bevorzugung von Laub- oder Mischwäldern gegenüber Nadelwäldern gefunden, die per Saldo ebenfalls mit einer positiven Zahlungsbereitschaft einhergeht. Rajmis et al. (2009) haben ebenfalls eine positive Zahlungsbereitschaft für artenreiche Mischwälder in Thüringen ermittelt. Ähnliches berichten Roovers et al. (2002), die bei ihrer Untersuchung von Besuchern eines stadtnahen Waldes in Belgien Präferenzen für gemischte Wälder fanden. Empfohlen wird (für Großbritannien von Bateman et al. 2011), die Präferenzen der Bevölkerung bei der Umsetzung von Waldbaumaßnahmen einzubeziehen. Die konkurrierenden Ziele „Erholung“ und „Ökologie“ sollten auf verschiedenen entsprechend geeigneten Flächen umgesetzt werden. Auch in der vorliegenden Befragung zeigte sich insgesamt eine Präferenz für Vielfalt: mehr Wald, keine Änderung der Flächengrößen, nicht weniger Unterwuchs, keinen größeren Anteil Nadelbäume (70 \%), aber höheres Erntealter. Nur in der stärkeren Beimischung von Bäumen aus anderen Ländern sieht die Bevölkerung mehr Nachteil als Vorteil und bewertet diese negativ.

\section{Weitere Leistungen des Waldes}

Eine weitere wichtige kulturelle Leistung des Waldes in Deutschland ist die Erholungsleistung. Nachfolgend soll zusätzlich auf ein paar wesentliche Eigenschaften dieser kulturellen Ökosystemleistung des Waldes eingegangen werden, da sie für die Bevölkerung sehr wichtig ist. Das Betreten des Waldes zur Erholung ist durch das Bundeswaldgesetz ausdrücklich gestattet (BWaldG §14). Des Weiteren ist die Erholungsleistung potenziell von den beschriebenen Waldumbaumaßnahmen betroffen. Elsasser und Weller (2013) zeigen, dass die mittlere Zahlungsbereitschaft von Waldbesuchern für Walderholung in Deutschland im Durchschnitt bei $32 € /$ Besucher/Jahr liegt. Im Hinblick auf potenzielle Steigerungsmöglichkeiten des Erholungswertes des Waldes wurden größere Unberührtheit und Natürlichkeit sowie Infrastrukturmaßnahmen zwar als Verbesserungsmöglichkeiten identifiziert, setzte man diese Verbesserungen alle um, würde sich die Zahlungsbereitschaft jedoch nicht substantiell erhöhen. Daraus lässt sich schlussfolgern, dass Veränderungen an den o. g. Merkmalen zwar Änderungen bei der Bewertung der Landschaft durch die Bevölkerung nach sich ziehen, nicht aber Änderungen bei der Bewertung der Erholungsleistung der Wälder durch 
die Bevölkerung. Letztere müssen also beim Vergleich der Strategien nicht zwingend berücksichtigt werden.

Auch zu weiteren Leistungen des Waldes liegen z. T. Ergebnisse aus der Literatur vor, die im Folgenden zu Vergleichszwecken sowie als Ergänzung referiert werden. Für die Klimaschutzleistung des Waldes, die durch die Maßnahmen im weitesten Sinne erhöht werden soll, gibt es sowohl sehr unterschiedliche Bewertungsansätze als auch sehr unterschiedliche Bewertungsergebnisse. Sie kann u. a. über die vermiedenen Schäden durch die vorhandene Senkenleistung des Waldes bewertet werden. Die globalen IPCC-Schätzungen zu Schadenskosten liegen zwischen -10 und +350 US\$/t Kohlenstoff (Yohe et al. 2007). Da sie auch in den negativen Bereich ragen, wird auch von teilweise positiven Wirkungen durch den Klimawandel ausgegangen. Das Umweltbundesamt empfiehlt, von durchschnittlichen Kosten in Höhe von $80 €$ /emittierter $\mathrm{t}$ $\mathrm{CO}_{2}$ auszugehen (UBA 2012; vgl. auch Abschn. 5.1.2.2). Volkswirtschaftlich sichtbar (und auch einzelwirtschaftlich spürbar) wird der Wert von Klimaschutzmaßnahmen im Europäischen Emissionszertifikatehandel bzw. den dortigen Preisen. Der Zertifikatspreis lag seit 2012 stets unter $10 € / t \mathrm{CO}_{2}$ (European Energy Exchange 2016). ${ }^{8}$ Der Preis pro Tonne $\mathrm{CO}_{2}$ wird zwar an der Börse ausgehandelt, die Menge an zu handelnden Zertifikaten wird aber politisch festgesetzt und verknappt. Die vergleichsweise geringe Höhe der Zertifikatspreise im Vergleich zu weltweiten Schadenskosten kann als Hinweis darauf gedeutet werden, dass diese politisch gesetzte Verknappung unter Effizienzgesichtspunkten nicht hinreichend ist. Die entsprechenden Preise sollten daher nicht mit dem volkwirtschaftlichen Nutzen einer eingesparten oder der Atmosphäre entzogenen Tonne $\mathrm{CO}_{2}$ verwechselt werden. Dieser volkswirtschaftliche Nutzen wäre durch einen Blick auf Zahlungsbereitschaften der Bevölkerung ermittelbar. Leider ist uns hierzu keine umfassende empirische Untersuchung bekannt. Einen Hinweis gibt eine (nicht repräsentative) Pilotuntersuchung aus Mannheim. Dabei führten Löschel et al. (2013) ein Experiment mit 202 Personen aus der Mannheimer Bevölkerung durch, bei dem diese Geld zur Verfügung gestellt bekamen, mit dem sie $\mathrm{CO}_{2}$-Zertifikate kaufen bzw. eventuell vorhandene Zahlungsbereitschaft zum Ausdruck bringen konnten. Dabei kristallisierte sich eine Median-Zahlungsbereitschaft von 0 heraus, die durchschnittliche Zahlungsbereitschaft lag bei $12 € / t \mathrm{CO}_{2}$. Im Anschluss an das Experiment wurden die Zertifikate tatsächlich gekauft und endgültig aus dem Markt entfernt. Die Zahlungsbereitschaft war stark abhängig (negativ) von aufgerufenen Preisen sowie dem Alter der Befragten und (positiv) von Einkommen und Bildungsgrad der Befragten sowie persönlichem Engagement für Klimaschutz.

Eine weitere wichtige Leistung des Waldes ist die Bereitstellung von (qualitativ hochwertigem) Wasser. Da es bereits einen Markt für Trinkwasser gibt, auf dem die Wasserversorgungsunternehmen als Anbieter auftreten, kann die Bewertung dieser Waldleistung

${ }^{8}$ Diese Schwelle wurde erst Ende Februar 2018 wieder überschritten, also lang nach Redaktionsschluss für dieses Buch. 
über die reduzierten Wasserbereitstellungskosten angenähert werden. So kommt man (in Frankreich) auf Einsparungen in Höhe von $138 € / J a h r / H a u s h a l t$, wenn 1 ha landwirtschaftlich genutzte Fläche durch 1 ha Wald ersetzt wird (Abildtrup et al. 2013). In Südamerika wurden von Núñez et al. (2006) ökonomische Vorteile für die regionalen Haushalte in Höhe von 49 bis 129,50€ (abhängig von der Wasser-Abundanz der jeweiligen Jahreszeit) pro Hektar Waldfläche berechnet.

Der Nutzen weiterer Leistungen geht teils auf (direkten oder indirekten) Gebrauch, teils aber auch auf Options-, Existenz- und altruistische Werte zurück, welche unter Umständen auch über weite Entfernungen als nützlich und schützenswert angesehen werden. Konkrete Beispiele dafür sind im ersten Fall ein lokaler Lawinen- und regionaler Hochwasserschutz und im zweiten Fall der von der eigenen Region losgelöste und trotzdem als wichtig bewertete Erhalt von Tier- und Pflanzenarten in anderen Ländern.

Zum Lawinenschutz haben Olschewski et al. (2012) in der Schweiz eine lokale Untersuchung durchgeführt, in der sie den Lawinenschutz des Waldes in der Gemeinde Andermatt analysierten. Die Zahlungsbereitschaft der Gemeindemitglieder für verschiedene Programme zum Schutz vor Lawinenereignissen lag dabei zwischen 110 und 390 US\$ (Einmalzahlung, Verwendung über mehrere Jahre für jeweilige Programmlaufzeit). Die Kosten für waldbauliche Maßnahmen zur Stärkung des Schutzwaldes waren in jedem Fall abgedeckt durch die Zahlungsbereitschaft. In einer weiteren Schweizer Studie wurde der Hochwasserschutz in Teilen der Alpen untersucht (Ryffel et al. 2014). Die Befragten aus dem Kanton Luzern waren bereit, verschiedene (kostenträchtige) Landnutzungswechsel in Kauf zu nehmen, um Hochwasserereignisse zu verhindern, etwa durch Schaffung von Überflutungsflächen. Da diese Leistungen jeweils nur von lokalem oder regionalem Interesse sind, wurden sie in diesem Projekt nicht näher analysiert.

\subsubsection{Aggregierte Ergebnisse der ökonomischen Bewertung der forstwirtschaftlichen Managementstrategien}

Peter Elsasser und Priska Weller

Im Vorstehenden wurden forstliche Maßnahmen zu abgestimmten Bündeln (Strategien) zusammengefasst (vgl. Abschn. 4.6.2) und mit forstbetrieblichen wie auch umweltökonomischen Methoden (Abschn. 4.2.2.4, 5.1.1) bewertet. Diese Ergebnisse liegen in unterschiedlichen Einheiten vor (Hektardurchschnitte auf der einen, mittlere Zahlungsbereitschaften pro Person auf der anderen Seite). Um direkt miteinander vergleichbar zu sein sowie um Aussagen über die Auswirkungen der vier untersuchten Strategien im Gesamtgebiet Deutschlands treffen zu können, müssen die jeweiligen Durchschnittsergebnisse auf die Gesamtfläche bzw. -bevölkerung hochgerechnet werden.

Eine solche Aggregation erfordert insbesondere über die physischen Auswirkungen der jeweiligen Strategien ergänzende Informationen, die teilweise nicht in der nötigen 
Differenzierung vorliegen und daher durch zusätzliche Annahmen überbrückt werden müssen. Diese Annahmen bewirken, dass die im Folgenden dargestellten Hochrechnungsergebnisse weniger belastbar sind als die Einzelergebnisse in den o. g. Bewertungskapiteln. Die wesentlichen Annahmen werden nachfolgend erläutert.

\section{Annahmen für die Hochrechnung der Bewertungsergebnisse im Bereich ,Wälder“}

Eine wesentliche Annahme betrifft den Zeitpunkt, für den die jeweiligen Bewertungsergebnisse gelten. Die in Abschn. 4.6 mit FoBeSiMo modellierten Waldwachstumssimulationen berücksichtigen zwar periodisch veränderliche Holzzuwächse und -entnahmen über den gesamten Betrachtungszeitraum; die zugehörigen Preise und Kosten wurden aber, wie forstüblich, als konstant angenommen (dies impliziert, dass für die Zukunft heutige Marktstrukturen unterstellt werden). Konsistent dazu wurde bei der umweltökonomischen Bewertung erfragt, wie die Bevölkerung die jeweils beschriebenen Waldveränderungen heute bewerten würde, und es wurde unterstellt, dass sich diese Bewertungen über die Zeit nicht ändern. Zusätzlich ist zu beachten, dass die physischen Auswirkungen einer veränderten Waldbewirtschaftung nur allmählich eintreten. Um dies bei der Hochrechnung zu berücksichtigen, wurde unterstellt, dass sämtliche physischen Umweltwirkungen erst am Ende der Betrachtungsperiode 2055 in voller Höhe auftreten und am Anfang null betragen; dazwischen wurde zur Vereinfachung linear interpoliert. Sowohl bei der betrieblichen als auch der umweltökonomischen Bewertung wird zudem keine Diskontierung durchgeführt (weder für zukünftige Zahlungs- noch für Nutzenströme). Berechnet werden jeweils jährliche Flussgrößen (jährliche Deckungsbeiträge sowie jährliche Zahlungsbereitschaften, jeweils im Durchschnitt des Betrachtungszeitraums).

Eine weitere Annahme wird für die Bewertung der Klimaschutzleistung des Waldes nötig, da Schätzungen des Nutzens einer der Atmosphäre entzogenen Tonne $\mathrm{CO}_{2}$ unterschiedlich begründet werden und über einen sehr weiten Bereich streuen (s. o.; zu Details siehe Ring et al. 2015 sowie Elsasser et al. 2015; Hartje et al. 2015). Des Weiteren kommen sie auch ganz unterschiedlichen Nutznießern zugute. Da die forstliche Senkenleistung im Europäischen Emissionshandelssystem (ETS) nicht anerkannt wird (und die Substitutionsleistung sich nur indirekt auf Preise auswirkt), beträgt der Handelswert der Klimaschutzleistung für die Forstbetriebe im ETS null. Einen Gegenpol bietet die Bewertung zu (weltweiten) Schadenskosten, die das deutsche Umweltbundesamt mit einem Richtwert von $80 € / t \mathrm{CO}_{2}$ beziffert (UBA 2012). Danach werden die durch die forstliche Klimaschutzleistung vermiedenen Schäden als volkswirtschaftlicher Nutzen dieser Leistung interpretiert, unabhängig davon, wo sie vermieden werden. Zwischen diesen Extremen liegen u. a. die Zahlungsbereitschaften nichtrepräsentativer Subgruppen der deutschen Bevölkerung (z. B. Löschel et al. 2013) wie auch die derzeitigen Marktpreise im Europäischen Emissionshandel. Um die Spannbreite möglicher Bewertungen und die unterschiedlichen Auswirkungen auf die verschiedenen Nutznießer aufzuzeigen, wird hier in einer ,mittleren“ Bewertungsvariante 
lediglich die Substitutionsleistung als Mengenbasis herangezogen und anhand des ETS-Durchschnittspreises der vergangenen fünf Jahre bewertet $\left(\approx 8,00 € / \mathrm{CO}_{2}\right) .{ }^{9}$ Damit wird sehr vereinfachend unterstellt, dass die Senkenleistung der Forst- und Holzwirtschaft mangels Anerkennung im ETS zwar für die Forstbetriebe finanziell irrelevant ist, die Substitutionsleistungen der Holzverwendung jedoch komplett ${ }^{10}$ den ETS-unterworfenen Sektoren der Volkswirtschaft zugutekommt, welche folglich den Ankauf entsprechender Zertifikate einsparen können. Diese Abschätzung wird zusätzlich von zwei weiteren Bewertungsvarianten eingerahmt: einerseits dem Handelswert der forstlichen Klimaschutzleistung $\left(0 € / t \mathrm{CO}_{2}\right)$ sowie andererseits dem UBA-Richtwert für weltweite Schadenskosten $\left(80 € / \mathrm{CO}_{2}\right.$ ). Als Mengenbasis werden in den letztgenannten beiden Fällen jeweils die durchschnittliche jährliche Speicherveränderung in Wald und Holz (Sequestrierung) und die jährliche stoffliche und energetische Substitution summiert, wie sie aus der Simulation mit FoBeSiMo hervorgehen.

Da im Choice-Experiment die meisten dort untersuchten Merkmale als Veränderungen des jeweiligen Status quo bewertet wurden, ist dieser zu quantifizieren. Generell werden dazu die jeweiligen Durchschnitte des jeweiligen Bundeslandes herangezogen. Für die einzelnen Merkmale gelten zudem folgende Setzungen:

- Die Merkmale „Größe von Feldern und Wäldern“ sowie „Unterwuchs im Wald“ werden an dieser Stelle nicht berücksichtigt, weil davon auszugehen ist, dass keine der hier untersuchten vier Strategien einen systematischen Einfluss auf das WaldFeld-Mosaik und auf die Schichtigkeit der Bestände ausübt.

- In Bezug auf die Baumartenmischung (Merkmal „Nadelbaumanteil“) liegen aus dem Choice-Experiment Zahlungsbereitschaften für einen 30-\%igen sowie einen 70-\%igen Nadelbaumanteil vor. Für die meisten Bundesländer impliziert der untere Wert eine Senkung des Nadelbaumanteils im Vergleich zu heute, der obere eine Steigerung (mit Ausnahme von Brandenburg, das derzeit $77 \%$ Nadelbäume aufweist, und dem Saarland mit $28 \%$ ). Im Normalfall werden daher Senkungen des Nadelbaumanteils anteilig anhand des unteren, Steigerungen anhand des oberen Schätzwertes der Zahlungsbereitschaft bewertet. Für die beiden Ausnahmen liegen jedoch jeweils zwei Schätzungen für eine Verkleinerung (Brandenburg) bzw. Vergrößerung (Saarland) des Nadelbaumanteils vor. Im Interesse einer vorsichtigen Bewertung wird hier jeweils lediglich der niedrigere Schätzwert der Zahlungsbereitschaft herangezogen.

\footnotetext{
${ }^{9}$ Nach den täglichen Preisangaben der Emissionsbörse EEX (www.eex.com/en/market-data/ emission-allowances/spot-market/european-emission-allowances) betrug der Preis einer EEA zwischen 01.11.2010 und 30.12.2015 im Durchschnitt 7,94 ₹ 8,00 €/t $\mathrm{CO}_{2}$.

${ }^{10}$ Dies führt zu einer Überschätzung, da ein erheblicher Teil der Substitutionswirkungen außerhalb der ETS-pflichtigen Sektoren anfallen dürfte. Gegenüber den erwähnten generellen Unsicherheiten bei der Bewertung der Klimaschutzleistung tritt dieses Problem allerdings in den Hintergrund.
} 
- Der Anteil von Bäumen aus anderen Ländern wird über den Anteil von Douglasien im Wald angenähert, da diese in der $\mathrm{BWI}^{3}$ (BMEL 2015) erfasst werden und den größten Anteil von Bäumen aus anderen Ländern ausmachen. Ausgehend von den in der $\mathrm{BWI}^{3}$ gemessenen Ist-Zuständen in den Bundesländern werden strategieinduzierte Änderungen in der Baumartenzusammensetzung zugunsten des Nadelbaumanteils einem steigenden Douglasienanteil zugerechnet, um diese in die Modellierungsergebnisse einzuhängen. Im Interesse einer vorsichtigen Schätzung wird maximal die Zahlungsbereitschaft für eine Verdopplung des Anteils von Bäumen aus anderen Ländern angesetzt (d. h., es wird ignoriert, dass die Strategien „Klimaschutz“ sowie „Bioenergie“ den Douglasienanteil bis 2055 mehr als verdoppeln). Eine Halbierung des Anteils von Bäumen aus anderen Ländern ist in keiner Strategie vorgesehen, deshalb werden die dafür vorliegenden Bewertungsergebnisse hier nicht verwendet.

- Aus dem Choice-Experiment liegen Bewertungen für Erhöhungen des Erntealters der Waldbestände vor. Aus den Ergebnissen der Wachstumssimulation lassen sich mit vertretbarem Aufwand aber nur Durchschnittsalter berechnen. Da bei regelmäßigem Altersaufbau Durchschnitts- und Erntealter im Verhältnis 1:2 stehen, wurde dieses Verhältnis vereinfachend hier unterstellt (d. h., Unregelmäßigkeiten im Altersaufbau der deutschen Wälder wurden vernachlässigt).

- Für Erhöhungen der Artenvielfalt im Wald liegen aus dem Choice-Experiment zwar Zahlungsbereitschaften vor, aber es ist nicht bekannt, wie die Artenvielfalt durch die jeweiligen Waldbewirtschaftungsstrategien physisch beeinflusst wird. Diese Einflüsse wurden mit Hilfe einer Expertenbefragung gutachtlich abgeschätzt. Im Ergebnis wurde vermutet, dass der Index der Artenvielfalt in der Referenzprojektion auf 80 Punkten verbleibt, in der Natur- und Umweltschutzstrategie bis zum Jahr 2055 auf 90 Punkte ansteigt und in der Bioenergiestrategie auf 70 Punkte absinkt; den Strategien „Klimaschutz“ sowie „Klimaanpassung“ wurden jeweils Zwischenwerte zugerechnet (85 bzw. 75 Punkte). Die zugehörigen Zahlungsbereitschaften wurden, wo nötig, linear interpoliert. Zudem wurde unterstellt, dass Verringerungen der Artenvielfalt (die nach der Experteneinschätzung bei den beiden letztgenannten Strategien zu erwarten sind) seitens der Bevölkerung wie Erhöhungen bewertet werden, sodass sich hier lediglich das Vorzeichen der Zahlungsbereitschaft umkehrt.

- Nutzungsverzichte (Merkmal „Ungenutzte Waldflächen“) wurden in der Simulation als separates Modul behandelt, das ggf. der Natur- und Umweltschutzstrategie zugerechnet wird. Da in diesem Modul ein Anteil von $10 \%$ ungenutzter Waldflächen vorgesehen ist und auch in der Befragung Nutzungsverzichte auf $10 \%$ der Waldflächen bewertet wurden, können die erhobenen Zahlungsbereitschaften ohne weitere Umrechnung verwendet werden. Ein vollständiger Verzicht auf ungenutzte Waldflächen ( $0 \%$ ungenutzte Waldflächen) ist in keiner der Strategien vorgesehen, sodass die Schätzung der entsprechenden Entschädigungsforderung in der vorliegenden Hochrechnung keine Verwendung findet.

- Die Vergrößerung der Waldfläche um $10 \%$ (Merkmal „Anteil Wald an der Landschaft") wurde als separates Modul behandelt und ggf. der Klimaschutz- 
strategie zugerechnet. Die entsprechende Zahlungsbereitschaft für eine 10-\%ige Waldflächenvergrößerung geht direkt aus der Befragung hervor. Eine Verkleinerung sieht keine der Strategien vor.

Schließlich erfolgt die Hochrechnung auf die erwachsene Bevölkerung der Bundesrepublik (Kinder und Jugendliche werden also im Interesse einer vorsichtigen Schätzung nicht berücksichtigt). Dies sind insgesamt 67,23 Mio. Menschen ab 18 Jahren (Destatis 2014).

\section{Aggregierte Ergebnisse}

Tab. 5.6 zeigt die aggregierten Ergebnisse für die Deckungsbeiträge aus Holznutzung sowie die drei unterschiedlichen Bewertungsvarianten für die Klimaschutzleistung, Tab. 5.7 die Hochrechnungsergebnisse für die durch die Strategien veränderten Umweltleistungen. Die in beiden Tabellen ausgewiesenen Summen können einander zum Zwecke der Interpretation gegenübergestellt werden, aber sie sollten nicht saldiert werden - zum einen aufgrund der konzeptionellen Differenzen der jeweiligen Bewertungsmaße (preisbasierte Bewertungen in Tab. 5.6 versus Konsumentenrenten in Tab. 5.7), und zum anderen wegen der unterschiedlichen Eigentumsverhältnisse an

Tab. 5.6 Hochrechnungsergebnisse der forstlichen Strategien: Monetäre Bewertung von Holzerträgen und Klimaschutzleistung im Durchschnitt der Jahre 2015-2055 (Mrd. €/Jahr). (Quelle: P. Weller)

\begin{tabular}{|c|c|c|c|c|}
\hline & Klimaschutz & Bioenergie & $\begin{array}{l}\text { Natur- und } \\
\text { Umweltschutz }\end{array}$ & $\begin{array}{l}\text { Klima- } \\
\text { anpassung }\end{array}$ \\
\hline $\begin{array}{l}\text { Waldbaulicher } \\
\text { Deckungsbeitrag }\end{array}$ & 2,92 & 2,74 & 2,55 & 2,39 \\
\hline Klimaschutzleistung ${ }^{\mathrm{a}}$ & $0|0,79| 12,33$ & $0|0,80| 11,59$ & $0|0,75| 12,28$ & $0|0,81| 11,49$ \\
\hline Summe & $2,92|3,72| 15,25$ & $2,74|3,54| 14,33$ & $2,55|3,29| 14,82$ & $2,39|3,20| 13,87$ \\
\hline \multicolumn{5}{|c|}{ Einschließlich Zusatzoption: Anteil Wald an der Landschaft/Aufforstung $(+10 \%)$} \\
\hline $\begin{array}{l}\text { Waldbaulicher } \\
\text { Deckungsbeitrag }\end{array}$ & $+0,03$ & - & - & - \\
\hline Klimaschutzleistunga & $0|+0,05|+1,20$ & - & - & - \\
\hline \multicolumn{5}{|c|}{ Einschließlich Zusatzoption: ungenutzte Waldflächen/Holznutzungsverzicht (10 \%) } \\
\hline $\begin{array}{l}\text { Waldbaulicher } \\
\text { Deckungsbeitrag }\end{array}$ & - & - & $-0,25$ & - \\
\hline Klimaschutzleistung ${ }^{\mathrm{a}}$ & - & - & $0|-0,29|-1,02$ & - \\
\hline $\begin{array}{l}\text { Summe inkl. Zusatz- } \\
\text { optionen }\end{array}$ & $2,95|3,80| 16,48$ & $2,74|3,54| 14,33$ & $2,30|2,99| 13,79$ & $2,39|3,20| 13,87$ \\
\hline
\end{tabular}

aUntere Variante: null (mangels Anerkennungsfähigkeit im ETS); mittlere Variante: Bewertung der Substitutionsleistung anhand mittleren ETS-Preises i.H.v. $8 € / t \mathrm{CO}_{2}$; obere Variante: Bewertung von Sequestrierungs- und Substitutionsleistung anhand von Schadenskosten i.H.v. $80 € / t \mathrm{CO}_{2}$ 
Tab. 5.7 Hochrechnungsergebnisse der forstlichen Strategien: Monetäre Bewertung veränderter Umweltleistungen (Mrd. €/Jahr). (Quelle: P. Weller)

\begin{tabular}{|c|c|c|c|c|}
\hline & Klimaschutz & Bioenergie & $\begin{array}{l}\text { Natur- und } \\
\text { Umweltschutz }\end{array}$ & Klimaanpassung \\
\hline $\begin{array}{l}\text { Anteil Wald an der } \\
\text { Landschaft }\end{array}$ & 0 & 0 & 0 & 0 \\
\hline $\begin{array}{l}\text { (Feldgröße, Unter- } \\
\text { wuchs) }\end{array}$ & - & - & - & - \\
\hline Nadelbaumanteil & $-3,86$ & $-5,14$ & $-0,58$ & $-0,68$ \\
\hline Erntealter & 3,97 & $-3,36$ & 5,02 & $-1,34$ \\
\hline Artenvielfalt & 3,69 & $-3,82$ & 3,82 & $-3,69$ \\
\hline $\begin{array}{l}\text { Ungenutzte Wald- } \\
\text { flächen }\end{array}$ & 0 & 0 & 0 & 0 \\
\hline $\begin{array}{l}\text { Bäume aus } \\
\text { anderen Ländern } \\
\text { (Douglasie) }^{\mathrm{a}}\end{array}$ & $0 \mid-12,09$ & $0 \mid-12,39$ & 0 & 0 \\
\hline Summe $^{\mathrm{a}}$ & $3,80 \mid-8,29$ & $-12,32 \mid-24,71$ & 8,26 & $-5,71$ \\
\hline $\begin{array}{l}\text { Zusatzoption: } \\
\text { Anteil Wald an der } \\
\text { Landschaft }(+10 \%)\end{array}$ & $4,26^{\mathrm{b}}$ & - & - & - \\
\hline $\begin{array}{l}\text { Zusatzoption: } \\
\text { ungenutzte Wald- } \\
\text { flächen }(10 \%)\end{array}$ & - & - & $-0,32$ & - \\
\hline $\begin{array}{l}\text { Summe inkl. } \\
\text { Zusatzoptionen }^{\text {a }}\end{array}$ & $8,06 \mid-4,03$ & $-12,32 \mid-24,71$ & 7,94 & $-5,71$ \\
\hline
\end{tabular}

${ }^{\mathrm{a}} \mathrm{Da}$ die (negativen) Bewertungen eines vergrößerten Anteils von Bäume aus anderen Ländern das Ergebnis stark dominieren, werden die Ergebnisse sowohl mit als auch ohne deren Berücksichtigung ausgewiesen

${ }^{b}$ Dieser Hochrechnung liegt lediglich die Zahlungsbereitschaft für eine Ausweitung der Waldfläche zugrunde. Aus welchen Baumarten diese besteht, wurde an dieser Stelle nicht thematisiert

den bewerteten Leistungen: Tab. 5.6 weist grundsätzlich monetäre Werte für private Güter aus (in Bezug auf die Bewertung der Klimaschutzleistung gilt dies allerdings nur für die untere und mittlere Bewertungsvariante), Tab. 5.7 hingegen monetäre Werte für öffentliche Güter. Eine Saldierung würde zwangsläufig die Frage nach wechselseitigen Kompensationszahlungen aufwerfen, die im vorliegenden Zusammenhang nicht Thema ist. Eine gemeinsame Betrachtung der verschiedenen Bewertungsergebnisse erlaubt aber erstmals eine grobe Gegenüberstellung der Kosten und des Nutzens der verschiedenen Strategien.

Wie aus dem oberen Teil von Tab. 5.6 ersichtlich, unterscheiden sich waldbauliche Deckungsbeiträge zwischen den Strategien um bis zu etwa $20 \%$, die Werte der Klimaschutzleistung um unter $10 \%$ (unabhängig davon, welches Verfahren jeweils zur 
Bewertung der Klimaschutzleistung verwendet wird). Ferner ist zu sehen, dass der Wert der Klimaschutzleistung in beiden Bewertungsansätzen, die den finanziellen Nutzen des Klimaschutzes für Betriebe in Deutschland auf Basis von ETS-Preisen abbilden, gegenüber den entsprechenden Deckungsbeiträgen stark in den Hintergrund tritt. Nur anhand einer Bewertung zu weltweiten Schadenskosten erscheint der Wert der Klimaschutzleistung substanziell. Er übertrifft dann sogar jeweils den Wert der Holzproduktion. ${ }^{11}$ Die Rangfolge der Strategien wird entsprechend stark durch die Deckungsbeiträge geprägt. In der Summe erweist sich die Klimaschutzstrategie als insgesamt leicht überlegen, die Klimaanpassungsstrategie bildet hingegen das Schlusslicht; die beiden anderen Strategien finden sich dazwischen.

Die beiden modularen Zusatzoptionen in den Strategien „Klimaschutz“ sowie „Naturund Umweltschutz“ modifizieren dieses Bild in unterschiedlicher Richtung. Die Aufforstung vergrößert sowohl die Deckungsbeiträge als auch die Klimaschutzleistung, wenn auch im ersteren Fall nur sehr schwach. Dabei ist zu bedenken, dass die Auswirkungen der Aufforstung innerhalb des nur 40-jährigen Betrachtungszeitraumes noch nicht voll zum Tragen kommen. Die Option „Nutzungsverzicht“" vermindert hingegen sowohl Deckungsbeiträge als auch Klimaschutzleistung spürbar. Rechnet man beide Zusatzoptionen den entsprechenden Strategien zu, so schlägt dies in der Summe auch auf die Rangfolge der vier Strategien durch: Einschließlich Zusatzoptionen verbleibt die Klimaschutzstrategie zwar an erster Stelle, die Natur- und Umweltschutzstrategie erweist sich dann aber sowohl in Bezug auf die Deckungsbeiträge als auch auf ihre Klimaschutzleistung allen anderen Strategien unterlegen.

Hinsichtlich ihres Einflusses auf den Nutzen der jeweiligen Umweltleistungen für die Bevölkerung unterscheiden sich die vier Strategien stärker (Tab. 5.7). Die Bioenergiestrategie wird insgesamt stark negativ bewertet, gefolgt von der Klimaanpassungsstrategie, welche insgesamt ebenfalls negativ bewertet wird. In beiden Strategien führen die vorgesehenen Änderungen am Nadelbaumanteil und am Ernte- bzw. Durchschnittsalter der Bestände wie auch die voraussichtlichen Auswirkungen auf die Artenvielfalt zu negativen Zahlungsbereitschaften (die als Entschädigungsforderungen interpretiert werden können). Dies gilt umso mehr, wenn man bei der Bioenergiestrategie zusätzlich die dort geplante Erhöhung des Douglasienanteils berücksichtigt: Da ein vergrößerter Anteil von Bäumen aus anderen Ländern in der Bevölkerung eine hohe Entschädigungsforderung auslöst, dominiert diese das Gesamtergebnis (obwohl sie hier nicht zur Gänze angerechnet worden ist; s. o.). Hingegen führen die Strategien „Klimaschutz“ sowie

\footnotetext{
${ }^{11}$ Die Bewertung zu weltweiten Schadenskosten ist gängig (s. z. B. den deutschen TEEB-Bericht zur Klimapolitik (Hartje et al. 2015)) und wurde hier im Interesse der Anschlussfähigkeit aufgenommen. Sie impliziert aber starke Annahmen (nämlich entweder, dass Deutschland für alle Folgeschäden hier emittierten Kohlenstoffs weltweit entschädigungspflichtig sei, oder, dass die deutsche Bevölkerung den Nutzen einer entsprechenden Schadensvermeidung innerhalb wie außerhalb Deutschlands gleich hoch bewerte).
} 
„Natur- und Umweltschutz“ per Saldo zu positiven Nutzenänderungen in Höhe von 3,80 bzw. 8,26 Mrd. €/Jahr (sofern bei der Klimaschutzstrategie auf eine starke Zunahme des Douglasienanteils verzichtet wird), die zurückzuführen ist auf die Erhöhung des jeweiligen Erntealters und der Artenvielfalt. Berücksichtigt man zusätzlich die beiden modular behandelten Maßnahmen „Erstaufforstung“ und „Nutzungsverzichte“, dann gleicht sich der Nutzen beider Strategien an und beträgt jeweils um $8 \mathrm{Mrd}$. €/Jahr. Auffällig ist dabei, dass Nutzungsverzichte auf $10 \%$ der existierenden Waldfläche auch aus Sicht der Bevölkerung den Nutzen der Natur- und Umweltschutzstrategie schmälern (vgl. Tab. 5.5).

Aufgrund der o. g. vielfältigen Unsicherheiten bei der Hochrechnung dieser Zahlen sollte deren jeweilige absolute Höhe nur mit äußerster Vorsicht interpretiert werden. Interessant erscheinen vielmehr die Relationen zwischen den Strategien. In der umweltökonomischen Bewertung erweisen sich insbesondere die Bioenergie-, abgeschwächt auch die Klimaanpassungsstrategie als wenig bevorzugenswert. Im Falle der Klimaanpassungsstrategie ist dies kongruent mit der Bewertung aus betriebswirtschaftlicher Sicht. Umgekehrt zeigen die Klimaschutz- wie die Natur- und Umweltschutzstrategie, je nach zugrundegelegten Annahmen, aus Sicht der Gesellschaft positiv bewertete Nutzenänderungen. Auch aus betriebswirtschaftlicher Sicht erwies sich die Klimaschutzstrategie im Vergleich zu den Alternativen als überlegen. Diese Strategie scheint eine konfliktarme Option zu bieten. Sie weist das stärkste Potenzial auf, sowohl aus betrieblicher als auch aus gesellschaftlicher Sicht akzeptiert zu werden.

\subsubsection{Diskussion und Ausblick zur ökonomischen Bewertung von Ökosystemleistungen der Landnutzung}

Jesko Hirschfeld und Julian Sagebiel

Bei den in Abschn. 5.1 vorgestellten Ergebnissen zu den jährlichen Zahlungsbereitschaften für unterschiedliche Landnutzungsstrategien ist $\mathrm{zu}$ berücksichtigen, dass es sich bei den hier ermittelten Zahlen um Näherungswerte handelt, die nicht als exakte Messwerte missverstanden werden sollten, die unmittelbar in politische Maßnahmen übersetzbar sind. Unsicherheiten bestehen in unterschiedlichem Ausmaß hinsichtlich der Formulierung der untersuchten Attribute, der modellhaft und annahmegestützt abgebildeten Wirkungszusammenhänge und in Bezug auf das Antwortverhalten der Befragten. Trotzdem sind die in Abschn. 5.1.2 und 5.1.3 vorgestellten Ergebnisse alles andere als irrelevant. Sie weisen auf erhebliche Zahlungsbereitschaften $u$. a. für eine Reduzierung von THG-Emissionen, für eine Verringerung der Nährstoffbelastung von Grund- und Oberflächengewässern, für mehr Wald und mehr Biodiversität hin - und damit auf starke Präferenzen in Richtung mehr Klima-, Umwelt- und Naturschutz.

Die damit verbundene Botschaft an die Politik liegt nicht darin, dass sie nun in Zukunft jährlich Steuermittel exakt in der hier ermittelten Höhe in zusätzliche Agrarumwelt- 
und Klimaschutzmaßnahmen investieren soll, sondern, dass es aus gesamtgesellschaftlicher Sicht politisch gewollt ist, dass solche Maßnahmen verstärkt ergriffen werden. Aus dieser gesellschaftlichen Präferenzäußerung ergibt sich ein Auftrag an die Politik, diese gesellschaftlichen Interessen in Ausgleich zu bringen mit den privaten, betriebswirtschaftlichen Interessen der land- und forstwirtschaftlichen Betriebe. Die ermittelten Zahlungsbereitschaften weisen dabei einerseits auf die relative Stärke der Präferenzen und Werthaltungen der Bevölkerung hin, andererseits umreißen sie Größenordnungen, was die bundesdeutsche Gesellschaft gegebenenfalls bereit wäre, zur Durchsetzung geeigneter Maßnahmen zu investieren - möglicherweise auch auf dem Wege einer entsprechenden Kompensation der Einbußen landnutzender Betriebe, die mit der Umsetzung von klima-, umwelt- und naturschutzorientierten Maßnahmen verbunden sein können.

Die Ergebnisse weisen außerdem darauf hin, welche der Strategien aus gesellschaftlicher Sicht robust positiv bewertet werden und welche hinsichtlich ihrer Akzeptanz ,auf der Kippe stehen“. Und ein Blick auf die einzelnen Zielgrößen zeigt, in welche Richtungen die einzelnen, hier zunächst aus Gründen der wissenschaftlichen Analyse klar unterscheidbar und unterschiedlich formulierten Strategien weiter optimiert werden könnten.

Die auf den Anbau von mehr Biomasse zur Erzeugung von mehr Bioenergie zur Substitution fossiler Energieträger orientierte Strategie wird zwar im Hinblick auf ihre Klimaschutzbeiträge als sehr positiv bewertet. Dem stehen aber gravierende negative Effekte auf die Nährstoffbelastung des Grundwassers, auf das Landschaftsbild und je nach konkreter Ausgestaltung der Anbauverfahren gegebenenfalls auch auf die Biodiversität auf landwirtschaftlichen Flächen gegenüber, sodass die Gesamtbewertung dieser Strategie ambivalent und damit aus gesellschaftlicher Sicht deutlich schlechter ausfällt als die anderen untersuchten Strategien.

Am anderen Ende der Skala erhalten die klimaschutzfokussierte sowie die naturund umweltschutzorientierte Strategie aus gesamtgesellschaftlicher Sicht eine deutlich positive Bewertung. Die erheblichen Nutzen, die in den Dimensionen „Klima- und Gewässerschutz“, „Landschaftsbild“ sowie „Erhaltung und Erhöhung der Biodiversität“" erzielt werden können, übertreffen die jeweiligen betriebswirtschaftlichen Einbußen deutlich. Würde die umwelt- und naturschutzorientierte Strategie kombiniert mit einer Vermehrung von Waldflächen und weiteren Klimaschutzmaßnahmen, die mit Naturschutzzielen gut zu verbinden wären (beispielsweise den Anbau von Paludikulturen auf wiedervernässten Moorflächen), ergäbe sich ein noch größerer positiver volkswirtschaftlicher Gesamteffekt (bis zu 6 Mrd. € pro Jahr) - dabei wäre eine vollständige Kompensation der damit verbundenen betriebswirtschaftlichen Einbußen der landwirtschaftlichen Betriebe bereits berücksichtigt.

Bei allen Ungenauigkeiten und Unsicherheiten der in den Abschn. 5.1.2 und 5.1.3 präsentierten Zahlen können nachstehende Aussagen gefolgert werden: Aus gesamtgesellschaftlicher Sicht ist deutlich mehr Klima-, Gewässer- und Naturschutz gut begründbar. Die Erzeugung von mehr Biomasse zur energetischen Verwertung wird dabei als ambivalent bis kritisch beurteilt. Eine Ausweitung von Waldflächen, eine 
Reduzierung des Einsatzes von Dünge- und Pflanzenschutzmitteln in der Landwirtschaft und eine Wiedervernässung von Mooren sowie weitere Maßnahmen zur Steigerung der Biodiversität in Land- und Forstwirtschaft werden deutlich befürwortet.

Die in der Befragungsstudie erhobenen Daten erlauben weitergehende Auswertungen hinsichtlich der regionalen Vorzüglichkeit einzelner Maßnahmen, indem Kosten und Nutzen räumlich explizit dargestellt und auf administrative Ebenen wie Landkreise oder Gemeinden interpoliert werden. Wenn beispielsweise eine Erhöhung des Waldanteils um $10 \%$ politisch gewollt ist, kann eine regionale Kosten-Nutzen-Analyse helfen, Gegenden zu identifizieren, in denen der Nettonutzen einer Aufforstung am höchsten ist. Die Veränderungen der bereitstellenden Ökosystemleistungen - insbesondere die Opportunitätskosten der Landwirte - werden im RAUMIS-Modell auf Landkreisebene berechnet. Ähnlich lassen sich auch die Zahlungsbereitschaften für die untersuchten kulturellen Ökosystemleistungen aufbereiten.

Abb. 5.6 zeigt beispielhaft die Ergebnisse einer regional differenzierteren Analyse: Ganz links sind die Waldanteile in den jeweiligen Landkreisen und kreisfreien Städten wiedergegeben. Es finden sich hohe Waldanteile (von z. T. über $50 \%$ der Landkreisfläche), vor allem im Südwesten (z. B. Schwarzwald), in der Mitte und im Südosten Deutschlands (u. a. Bayerischer Wald). Sehr niedrige Waldanteile von z. T. unter $1 \%$ der Landkreisfläche finden sich vor allem in Nordwestdeutschland.

Die mittlere Karte von Abb. 5.6 zeigt die Zahlungsbereitschaft pro Kopf für eine Erhöhung des Waldanteils im unmittelbaren Umfeld des Wohnortes (15 km-Radius), basierend auf den Ergebnissen aus Abschn. 5.1.2.3. Deutlich erkennbar sind hohe ProKopf-Zahlungsbereitschaften vor allem in waldarmen Regionen. Aggregiert man die regionalen Pro-Kopf-Zahlungsbereitschaften mit den jeweiligen Einwohnerzahlen, ergeben sich regional stark differenzierte Gesamtzahlungsbereitschaften (vgl. rechte Karte in Abb. 5.6). Durch eine entsprechende regionale Differenzierung der Maßnahmen zur Erhöhung des Waldanteils kann der gesamtgesellschaftliche Nutzen also gegenüber einer bundeseinheitlichen Lösung deutlich erhöht werden.

Die regional differenzierte Analyse der Nutzen von Landnutzungsmaßnahmen ist hier exemplarisch für die Erhöhung von Waldanteilen dargestellt. Sie lässt sich prinzipiell auch für andere Maßnahmen durchführen, beispielsweise für eine Begrenzung des Maisanteils, eine Erhöhung der Biodiversität oder die Begrenzung von Nährstoffbilanzüberschüssen. Sie kann auf der anderen Seite den ebenfalls regional differenzierten Kosten gegenübergestellt werden. Diese Analysen zeigen, dass eine regionale Differenzierung von Maßnahmen, die sich an den räumlichen Mustern der Verteilung von Nutzen und Kosten orientiert, für eine gesamtgesellschaftlich optimale Lösung unabdingbar ist.

Der für die Analyse von Landnutzungsstrategien in Deutschland innovative Ansatz regional differenzierter, ökologisch erweiterter Nutzen-Kosten-Analysen konnte methodisch und empirisch erarbeitet und für einzelne Attribute exemplarisch umgesetzt werden. Die erhobenen Daten bieten über das hinaus noch erhebliche zusätzliche Potenziale für weitere räumlich differenzierte Auswertungen, die durch weitergehende Forschungsarbeiten erschlossen werden können. 

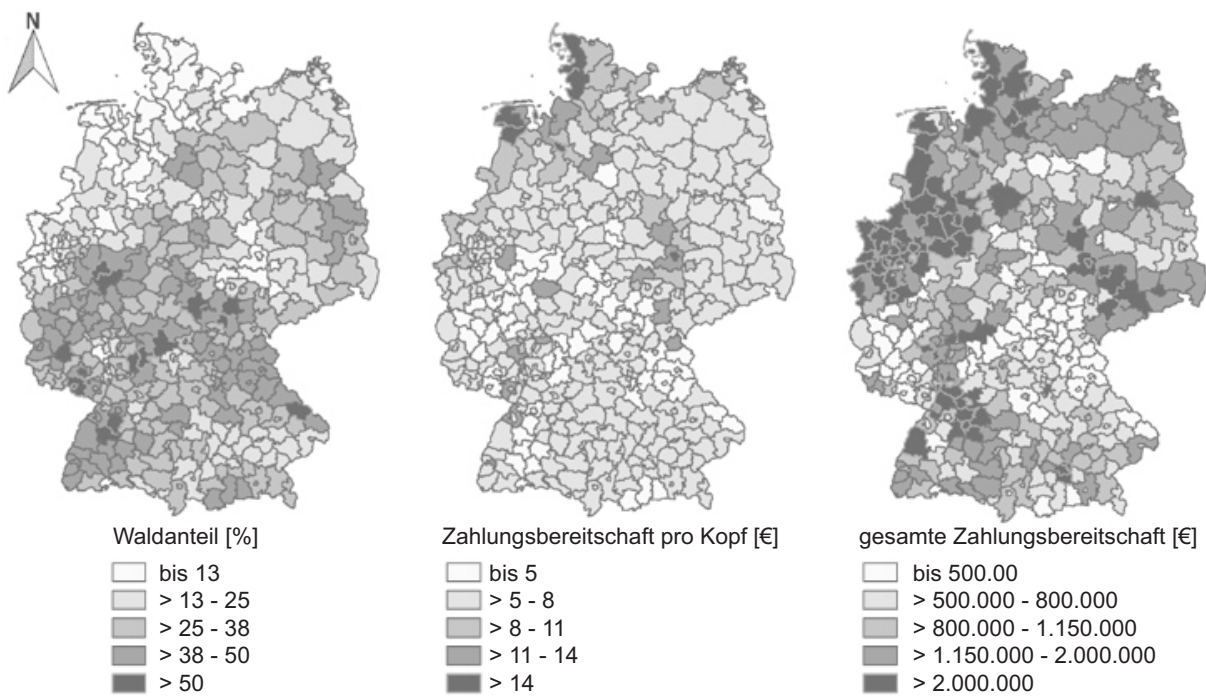

Quellen: BBSR, BKG, Statistisches Bundesamt

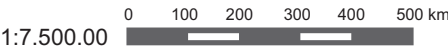

Abb. 5.6 Waldanteil und Marginale Zahlungsbereitschaft für Wald auf Kreisebene

\subsection{Institutionelle Gestaltungsoptionen für eine nachhaltige Landnutzung}

Ulrike Grabski-Kieron und Mathias Raabe

\section{Zusammenfassung}

Landnutzungsentscheidungen orientieren sich nicht nur an gegebenen Standortpotenzialen, sondern unterliegen auch Rahmensetzungen des Umwelt- und Planungsrechts, den Anreizsetzungen verschiedener Förderpolitiken sowie den Einflüssen privater und öffentlicher Akteure und Institutionen durch Aushandlungsprozesse und eigene Strategieentwicklungen (Governance). Diese Regelungs- und Steuerungssysteme der Landnutzung gilt es unter den Vorzeichen des Klimawandels anzupassen und weiterzuentwickeln, um den Herausforderungen des Klimaschutzes und der Klimaanpassung sektorspezifisch und sektorübergreifend begegnen $\mathrm{zu}$ können. Hierzu erfolgt eine umfängliche planungswissenschaftliche Evaluierung der vorhandenen Steuerungselemente, ergänzt durch Literatur- und Dokumentenrecherchen sowie die Ergebnisse der nationalen und regionalen Stakeholder-Beteiligung. Darauf aufbauend können sowohl maßnahmenspezifische als auch übergeordnete institutionelle Gestaltungsoptionen für das Management einer nachhaltigen Landnutzung abgeleitet werden. 


\subsubsection{Einführung, Zielsetzung und methodische Vorgehensweise}

Landnutzungsentscheidungen orientieren sich nicht nur an gegebenen Standortpotenzialen. Sie sind vielmehr Ergebnis komplexer Steuerungs- und Entscheidungsprozesse, die sich auf verschiedenen Handlungsebenen entfalten. Im örtlichen, regionalen, nationalen und auch im europäischen Bezugsrahmen unterliegen Landnutzungsentscheidungen Rahmensetzungen des Umwelt- und Planungsrechts und sind Einflüssen verschiedener sektoraler Anreiz- und Förderpolitiken ausgesetzt. Gleichzeitig sind sie Ausdruck kooperativer, strategischer wie auch umsetzungsorientierter Aushandlungsprozesse zwischen öffentlichen und privaten Akteuren. Diese komplexen hybriden, flexiblen Governancestrukturen (vgl. Fürst und Scholles 2008a, b) bilden zusammen die aktuellen Regelungs- und Steuerungssysteme der Landnutzung (vgl. Abb. 5.7).

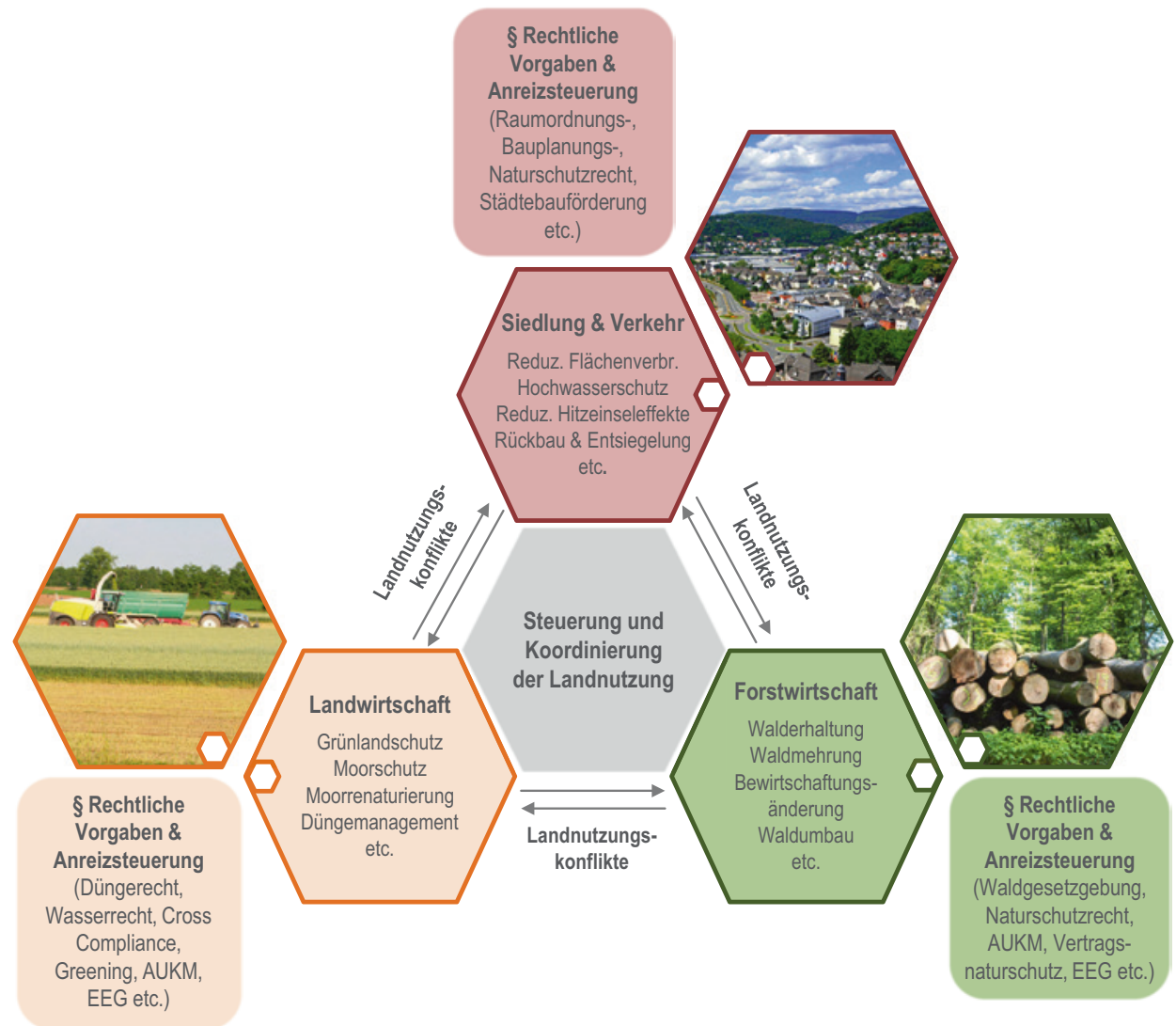

Abb. 5.7 Landnutzungssteuerung im Klimawandel 
Die sich abzeichnenden klimatischen Veränderungen und die dadurch notwendigen Anpassungen in der Landnutzung stellen neue Herausforderungen an ein nachhaltiges Landnutzungsmanagement. Insofern stellt sich die Frage, ob die aktuellen Regelungsund Steuerungssysteme der Landnutzung diesen Herausforderungen gerecht werden und hinreichend geeignet sind, um die herausgearbeiteten Landnutzungsstrategien und die dort umfassten Maßnahmen umzusetzen. Um institutionelle Gestaltungsoptionen für ein nachhaltiges Landnutzungsmanagement herauszuarbeiten, wurden zusätzlich zur Akteursbeteiligung die umwelt- und planungsrechtlichen sowie anreiztechnischen Steuerungsmöglichkeiten der Landnutzung in den Mittelpunkt gestellt. So soll ein Beitrag für das Verständnis der Wechselbeziehungen zwischen Landnutzungsentscheidungen einerseits und rechtlichen Rahmenbedingungen andererseits geleistet werden. Gleichzeitig wird gewährleistet, dass in die Erarbeitung nachhaltiger Landnutzungsstrategien über die naturwissenschaftlichen Aspekte hinaus planungsbezogene und sozioökonomische Steuerungsbelange mit einfließen.

Das Ziel ist, übergeordnete institutionelle Gestaltungsoptionen $\mathrm{zu}$ formulieren sowie maßnahmespezifische Gestaltungsoptionen für ein nachhaltiges Landnutzungsmanagement abzuleiten. Die Betrachtung institutioneller Gestaltungsoptionen erfolgt für die Maßnahmen, die in Kap. 4 zu Landnutzungsstrategien gebündelt und analysiert wurden. Es wird nicht der Frage nachgegangen, ob eine spezifische Maßnahme sinnvoll ist oder nicht, sondern welche Handlungsoptionen bestehen, wenn die Maßnahme (als Teil einer der formulierten Strategien) zur Umsetzung kommt bzw. befördert werden soll. Hierzu werden die Maßnahmen hinsichtlich der rechtlich-instrumentellen und politisch-gesellschaftlichen Umsetzbarkeit bewertet (vgl. Abb. 5.8).

Die einschlägigen Regelungsinhalte, Handlungsansätze und Instrumentarien wurden zunächst im Rahmen einer planungswissenschaftlichen Evaluierungsforschung auf Basis eines Kriteriensystems in ihrer Adaptionsfähigkeit an sich wandelnde Anforderungen wie auch hinsichtlich ihrer Potenziale zur Weiterentwicklung einer Bewertung unterzogen. Berücksichtigung fanden dabei alle diejenigen Instrumente, die als rechtliche Regelungssachverhalte, Verfahren, Anreizmechanismen, Strategien, Konzepte und informelle Kooperationsformen zu einer Steuerung, Einflussnahme oder zu einer konkreten Umsetzung von Entscheidungen in der Landnutzung führen und denen in formal-rechtlichen wie auch informellen Governance-Kontexten strukturell-gestaltende und funktional-differenzierende Wirkungen in der Raumnutzung zukommen. Als Kriterien zur Bewertung der vorhandenen Regelungssysteme wurden herangezogen:

- die Passgenauigkeit: Bewertung der Einflussnahme rechtlicher Regelungen oder Instrumente auf Klimaschutz und/oder Klimaanpassung;

- der Umsetzungsbezug: Bewertung der Wirkungsweise einzelner Instrumente mit Bezug auf die Frage, welche Impulse vom Instrument/seinem Steuerungsansatz und/oder 
Ziel 1: Ableitung übergeordneter institutioneller Gestaltungsoptionen für ein nachhaltiges Landnutzungsmanagement

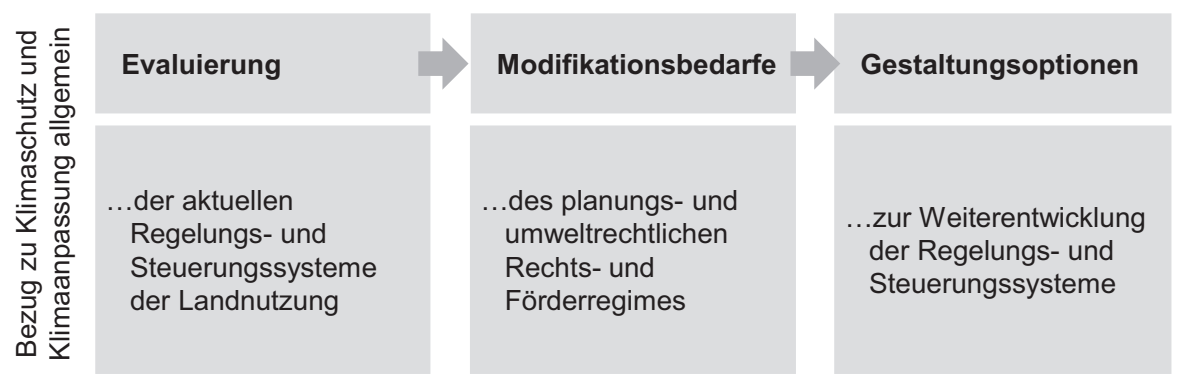

Ziel 2: Ableitung maßnahmenspezifischer institutioneller Gestaltungsoptionen für ein nachhaltiges Landnutzungsmanagement

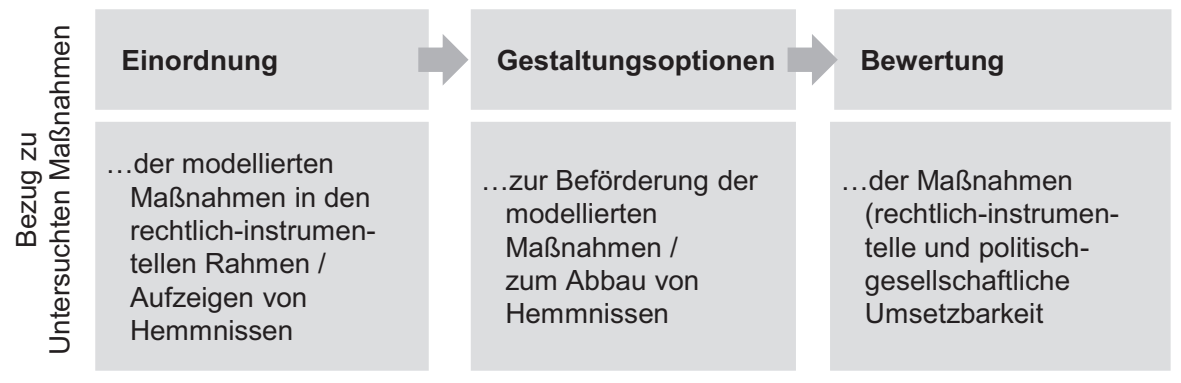

Einbettung in regionalen und nationalen Beteiligungsprozess

Abb. 5.8 Zielsetzung und methodisches Vorgehen

seinem Einflusselement ausgehen, um Klimaschutz-/Klimaanpassungsmaßnahmen im Landnutzungsmanagement umzusetzen ${ }^{12}$;

- die Bindungswirkung: Bewertung der rechtlichen Bindungswirkung eines Regelungsund Steuerungsansatzes/eines Einflusselements zur Umsetzung von Maßnahmen für Klimaschutz/Klimaanpassung in der Landnutzung;

- die Umsetzungsrelevanz: Bewertung der theoretischen und praktischen Bedeutung, die einem Regelungs- und Steuerungsansatz/Einflusselement für eine nachhaltige, klimawandelangepasste Landnutzung zukommt.

\footnotetext{
${ }^{12}$ Beispielsweise unmittelbar wirksame Verbote versus Festlegungen in Raumordnungsplänen, die der Konkretisierung durch nachgelagerte Planungen bedürfen.
} 
Zudem wurde festgehalten, welche Zielgruppen von einzelnen Instrumenten und Regelungsinhalten angesprochen werden und welche Bezüge $\mathrm{zu}$ den modellierten Maßnahmen bestehen. Die Ergebnisse der eigenen fachlich-inhaltlichen Analyse wurden mit Aussagen der begleitenden rechts- und planungswissenschaftlichen Literatur- und Dokumentenrecherche abgeglichen und ergänzt. Darüber hinaus flossen Kernaussagen der nationalen und regionalen Stakeholder-Beteiligung sowie eines Experten-Workshops in die Analyse mit ein, was eine weitere Validierung der Ergebnisse ermöglichte. Die abgeleiteten Handlungsoptionen zur Modifikation einzelner Steuerungsinstrumente wurden ferner auf ihre rechtliche Umsetzbarkeit hin juristisch überprüft ${ }^{13}$ und mit den sektoralen Projektpartnern fachlich abgestimmt. Somit ergibt sich ein ganzheitlicher Bewertungsansatz, in den mehrere methodische Bausteine einfließen.

\subsubsection{Ergebnisse der planungswissenschaftlichen Evaluierung}

Die Ergebnisse der planungswissenschaftlichen Evaluierung der landnutzungsrelevanten Regelungs- und Steuerungssysteme des Planungs- und Umweltrechts sowie der wichtigsten anreiztechnischen Förderpolitiken dienten als Ausgangsbasis der weiteren Arbeiten und waren handlungsleitend bei der Ableitung institutioneller Gestaltungsoptionen.

Im Kern kann festgehalten werden, dass das deutsche Planungs- und Umweltrecht bereits durch eine weitgehende rechtliche Verankerung von Klimaschutz und Klimaanpassung gekennzeichnet ist und in den vergangenen Jahren ein breit aufgestellter Instrumentenkasten mit unterschiedlichen Instrumententypen entwickelt wurde. Mit deren Hilfe können bereits heute klimawandelangepasste Raumstrukturen entwickelt werden (vgl. Abb. 5.9). Gleichzeitig kann konstatiert werden, dass in Teilen eine Weiterentwicklung einzelner Instrumente im Sinne des Klimaschutzes und der Klimaanpassung notwendig sein wird und in verschiedenen Bereichen deutliche Vollzugs- und Umsetzungsdefizite vorhandener Instrumente festzustellen sind. Die hohe Bedeutung informeller Handlungs- und Planungsansätze hat sich bestätigt. Zudem sind im Ergebnis der Analyse Instrumente in den Vordergrund gerückt, die in fachlichen Diskursen bisher nur vereinzelt mit der Umsetzung von Klimaschutz- und Klimaanpassungsmaßnahmen in Verbindung gebracht werden. Sektorübergreifend hat sich gezeigt, dass das Zusammenwirken einzelner Instrumente und die konkrete Ausgestaltung der Governance-Systeme (Kooperation und Koordination zwischen Akteuren, Institutionen) für die erfolgreiche Umsetzung klimawandelrelevanter Maßnahmen von zentraler Bedeutung sind.

\footnotetext{
${ }^{13}$ Zusammenarbeit mit dem Zentralinstitut für Raumplanung (ZIR) an der Universität Münster.
} 


\begin{tabular}{|c|c|c|}
\hline \multicolumn{3}{|c|}{ Unterschiedliche Instrumententypen zur Steuerung der Landnutzung } \\
\hline$€$ & $\begin{array}{l}\text { Anreize \& } \\
\text { Fördermittel }\end{array}$ & $\begin{array}{l}\text { zum Beispiel... } \\
\text { - Gemeinsame Agrarpolitik (Direktzahlungen, CC, Greening, AUKM) } \\
\text { - Gemeinschaftsaufgabe Agrarstruktur und Küstenschutz } \\
\text { - Städtebauförderung } \\
\text { - Innovative Finanzierungsmodelle (z. B. MoorFutures) }\end{array}$ \\
\hline $\mathrm{i}$ & $\begin{array}{l}\text { Information \& } \\
\text { Beratung }\end{array}$ & $\begin{array}{l}\text { zum Beispiel... } \\
\text { - Düngeberatung } \\
\text { - Waldbauliche Handlungsempfehlungen } \\
\text { - Infrastrukturfolgekostenberechnungen } \\
\text { - Brachflächenmonitoring }\end{array}$ \\
\hline$\S$ & Rechtliche Vorgaben & $\begin{array}{l}\text { zum Beispiel... } \\
\text { - Walderhaltungsgebot } \\
\text { - Naturschutzrechtliche Grünlandumbruchverbote } \\
\text { - Kompensation von Eingriffen } \\
\text { - Umweltverträglichkeitsprüfung }\end{array}$ \\
\hline 贯 & $\begin{array}{l}\text { Kooperation \& } \\
\text { Zusammenarbeit }\end{array}$ & $\begin{array}{l}\text { zum Beispiel... } \\
\text { - Landwirtschaft \& kommunale Wasserversorger (Düngung) } \\
\text { - Interkommunale Kooperation } \\
\text { - Forstwirtschaftliche Zusammenschlüsse } \\
\text { - Public Private Partnership }\end{array}$ \\
\hline$\nwarrow \uparrow \pi$ & $\begin{array}{l}\text { Planerische } \\
\text { Steuerung }\end{array}$ & $\begin{array}{l}\text { zum Beispiel... } \\
\text { - Räumliche Gesamtplanung (z. B. Regionalplanung, Bauleitplanung) } \\
\text { - Fachplanung (z. B. Landschaftsplanung, Verkehrsplanung) } \\
\text { - Stadtplanung/Quartiersplanung } \\
\text { - Flurbereinigung }\end{array}$ \\
\hline$€ / \S$ & $\begin{array}{l}\text { Ökonomische \& } \\
\text { fiskalische } \\
\text { Instrumente }\end{array}$ & $\begin{array}{l}\text { zum Beispiel... } \\
\text { - Finanzausgleich } \\
\text { - Entfernungspauschale } \\
\text { - Emissionshandel } \\
\text { - Flächenhandel (perspektivisch) }\end{array}$ \\
\hline
\end{tabular}

Abb. 5.9 Unterschiedliche Instrumententypen zur Steuerung der Landnutzung

\subsection{3 Übergeordnete Gestaltungsoptionen für ein nachhaltiges Landnutzungsmanagement}

Bevor maßnahmenbezogene Handlungsoptionen thematisiert werden, soll zunächst auf sektorübergreifende Gestaltungsoptionen für ein nachhaltigeres Landnutzungsmanagement in Deutschland eingegangen werden. Diese greifen die Notwendigkeit auf, Landnutzung effektiver als bisher zu steuern und zu koordinieren und dabei die Herausforderungen des Klimawandels stärker als bisher zu berücksichtigen. 


\section{Effektivere Steuerung und Koordinierung der Landnutzung durch räumliche Gesamtplanung}

Im Kontext der Ausgestaltung eines nachhaltigen Landnutzungsmanagements ist auf die Notwendigkeit hinzuweisen, stärker als bisher die integrierenden und koordinierenden Potenziale der räumlichen Gesamtplanung auszunutzen. Diese kann auf überörtlicher Ebene im Rahmen der Landesentwicklungs- und Regionalplanung sowie dann auf Ebene der Kommunalplanung wesentlich dazu beitragen, die im Zuge des Klimawandels und der Energiewende zunehmenden Flächennutzungskonflikte frühzeitig zu erkennen und koordinierend auf diese einzuwirken. Zudem kann sie Klimaschutzmaßnahmen ${ }^{14}$ sowie Maßnahmen zur Strategie „Klimaanpassung“15 vorbereiten und entsprechende Flächen sichern.

Bei der Umsetzung einer klimawandelgerechten Raumordnung kommt der Regionalplanung eine Schlüsselfunktion zu. Dies liegt darin begründet, dass sie aufgrund ihrer im Vergleich zur kommunalen Bauleitplanung größeren Maßstäblichkeit bei gleichzeitig hinreichendem räumlichen Konkretisierungsgrad die am besten geeignete Handlungsebene zur Erarbeitung überörtlicher und querschnittbezogener Strategien und Maßnahmen darstellt. Ihre theoretische Relevanz wird in der Planungspraxis - länderweise unterschiedlich - durch eine begrenzte finanzielle und personelle Ausstattung eingeschränkt. Eine explizite Aufarbeitung klimaschutz- oder klimaanpassungsrelevanter Belange vollzieht sich jedoch mehr und mehr.

Vor dem Hintergrund der vielfältigen Anforderungen, die der Klimawandel an die künftige Ausgestaltung der Landnutzung stellt, erscheint auch eine stärkere Einbeziehung und bessere Verknüpfung der formal-rechtlichen Regionalplanung mit informellen Planungs- und Handlungsansätzen ${ }^{16}$ notwendig (s. u.). Diese sind dazu in der Lage, klimawandelbezogene Themen und Inhalte zu transportieren und formalrechtliche Planungen vorzubereiten. Vor diesem Hintergrund ist auch die Forderung der Akademie für Raumforschung und Landesplanung (vgl. Spiekermann und Franck 2014, S. 21) zu unterstützen, die klassische Regionalplanung zu einer strategischen Regionalplanung weiterzuentwickeln und um Aufgaben und Handlungsmöglichkeiten des Regionalmanagements zu erweitern. Dies sollte u. a. die Ausstattung der Regionalplanung mit eigenen investiven Mitteln sowie ausreichenden Kapazitäten und Kompetenzen, das verstärkte Arbeiten mit Szenarien und die bereits angesprochene intensivere Berücksichtigung informeller regionaler Entwicklungsprozesse umfassen.

\footnotetext{
${ }^{14}$ Etwa die Umsetzung einer flächensparenden und verkehrsvermeidenden Siedlungs- und Verkehrsflächenentwicklung, die räumliche Vorsorge für eine klimaverträgliche Energieversorgung oder auch die raumordnerische Sicherung von $\mathrm{CO}_{2}$-Senken.

${ }^{15}$ Beispielsweise im Hochwasser- und Küstenschutz sowie bei der Verringerung städtischer Hitzefolgen.

${ }^{16}$ Dies können etwa regionale Entwicklungskonzepte sein oder regionale Klimaschutzkonzepte sowie themenspezifische Konzepte zum Einsatz erneuerbarer Energien.
} 


\section{Verstärkte Berücksichtigung von Klimawandelfolgen in der räumlichen Planung}

Im Rahmen von Umweltverträglichkeitsprüfungen für Projekte sowie für Pläne und Programme nach Umweltverträglichkeitsprüfungsgesetz (UVPG) in Verbindung mit dem Raumordnungsgesetz (ROG) und dem Baugesetzbuch (BauGB) wird sichergestellt, dass bei öffentlichen und privaten Vorhaben zur wirksamen Umweltvorsorge nach einheitlichen Grundsätzen die Auswirkungen auf die Umwelt frühzeitig und umfassend ermittelt, beschrieben und bewertet und die Ergebnisse der durchgeführten Umweltprüfungen in planerischen Entscheidungen berücksichtigt werden. Dabei ist festzustellen, dass das UVPG in seiner jetzigen Fassung keine Klimafolgenprüfung vorsieht, sondern lediglich die Auswirkungen des prüfpflichtigen Vorhabens auf das Klima untersucht werden.

Zur stärkeren Berücksichtigung der Folgen des Klimawandels auf Planungsprozesse bedarf es insofern der Etablierung einer Klimafolgenverträglichkeitsprüfung ${ }^{17}$, in deren Rahmen im Sinne eines Perspektivenwechsels künftig die Folgen des Klimawandels bei der Projektplanung und Planaufstellung Berücksichtigung finden. Da bei der Planung einzelner Projekte (z. B. eines Kraftwerkes) entsprechende Risiken bereits über das Anlagenrecht hinreichend Berücksichtigung finden, ist die Etablierung einer Klimafolgenverträglichkeitsprüfung in erster Linie für die Strategische Umweltprüfung (SUP) angezeigt. Dabei sollte ein solches Prüfverfahren bestenfalls in die SUP integriert werden, um die Planungspraxis nicht durch ein weiteres Prüfinstrument zu überfrachten (so auch MKRO 2013, S. 34). Besondere Relevanz erhielte das Prüfverfahren auf regionaler Ebene bei der Aufstellung regionaler Raumordnungspläne. Hier könnten die Klimawandelfolgen bereits auf überörtlicher Ebene ermittelt und bewertet werden, was gleichzeitig die informatorische Funktion der Regionalplanung deutlich aufwerten würde.

\section{Weiterentwicklung der Landschaftsplanung}

In Bezug auf die fachplanerische Aufbereitung und Bearbeitung klimawandelrelevanter Aufgaben und Inhalte ist festzuhalten, dass in Deutschland keine eigenständige KlimaFachplanung existiert, in deren Rahmen Belange des Klimaschutzes und der Klimaanpassung hinreichend Berücksichtigung finden. Der Landschaftsplanung wird in diesem Zusammenhang das Potenzial zugesprochen, wichtige Ermittlungs-, Koordinierungsund Abwägungsaufgaben zu übernehmen (vgl. Wilke et al. 2011, S. 128 ff.), was jedoch aufgrund der geltenden Rechtslage nicht zu erwarten ist (vgl. Reese et al. 2010, S. 394). Es zeigt sich, dass Belange des Klimawandels zwar vermehrt Eingang in die Landschaftsplanungspraxis auf regionaler Ebene finden, jedoch erfolgt bisher die Bearbeitung in sehr unterschiedlicher Intensität, wobei eine stringente, systematische und strukturierte Bearbeitung zurzeit nicht stattfindet (vgl. Radtke et al. 2015). Dabei könnte sie bei der Erfüllung neuer (und alter) Aufgaben wesentliche Beiträge zur Ausgestaltung

\footnotetext{
${ }^{17}$ Das sogenannte „Climate Proofing“, zurückgehend auf Kabat et al. (2005).
} 
eines klimawandelgerechten und nachhaltigen Landnutzungsmanagements leisten, zum Beispiel durch

- die Bewertung der Senkenfunktion von Ökosystemen (Quantifizierung des Kohlenstoffgehaltes),

- die räumliche Steuerung und Bündelung von Kompensationsmaßnahmen sowie Agrarumwelt- und Klimamaßnahmen (AUKM),

- die Informationsbereitstellung zur Erarbeitung von Klimafolgenverträglichkeitsprüfungen sowie

- die Zusammenführung fachbehördlicher Einzelinteressen zu einem integrativen landschaftlichen Ansatz.

Hierzu bedürfte es rechtlicher und prozessualer Modifikationen, um die Landschaftsplanung im Sinne einer mehrstufigen, integrierten Umweltplanung auszugestalten, die schutzgutübergreifend Ermittlungs-, Koordinierungs- und Abwägungsaufgaben übernehmen kann und diese mit entsprechender Bindungswirkung zur Umsetzung führt. Im Einzelnen würde die Weiterentwicklung der Landschaftsplanung u. a. folgende Modifikationen erfordern (vgl. vertiefend auch Marschall et al. 2012):

- Erhöhung der rechtlichen Bindungswirkung landschaftsplanerischer Festlegungen; ${ }^{18}$

- Festschreibung regelmäßiger Überprüfungsintervalle der Landschaftspläne und Wiedereinführung der Pflicht zur flächendeckenden Landschaftsplanung auf örtlicher Ebene;

- klarstellende Ergänzung der Klimaanpassung als Ziel der Landschaftsplanung im BNatSchG;

- vermehrter Einsatz von Szenarien und (Klima-)Projektionen;

- Sicherstellung einer ausreichenden Ressourcenausstattung der Landschaftsplanung.

So ließe sich ein bestehendes und grundsätzlich erprobtes und bewährtes Instrumentarium dazu nutzen, ein rechtliches Planungsinstrument zu etablieren, welches der Tatsache Rechnung trägt, dass die Risiken des Klimawandels - abgesehen von Extremwetterereignissen mit nur kurzzeitig erhöhtem Problembewusstsein - oftmals keinen unmittelbaren Handlungsdruck erzeugen und somit meist keine hinreichende planerische Berücksichtigung finden.

\section{Weiterentwicklung des Kompensationsflächenmanagements}

Das Bundesnaturschutzgesetz (BNatSchG, § 13) besagt, dass Eingriffe in Natur und Landschaft durch Kompensationsmaßnahmen auszugleichen sind, sofern sie nicht ver-

\footnotetext{
${ }^{18}$ Etwa durch eindeutigere Berücksichtigungs- und Beachtenspflichten sowie Beteiligungs-, Benehmens- und Einvernehmensvorbehalte.
} 
meidbar sind. Die Realisierung dieser Kompensationsmaßnahmen erfolgt aufgrund der allgemeinen Flächenknappheit zu einem Großteil auf landwirtschaftlichen Nutzflächen. Diese weisen infolgedessen deutliche Flächenverluste auf, zusätzlich zur ohnehin hohen Flächeninanspruchnahme durch Siedlungs- und Verkehrsflächen (vgl. Abschn.2.2). Dadurch wird zum einen das landwirtschaftliche Produktionspotenzial eingeschränkt, zum anderen sind deutliche Umsetzungsdefizite bei der Planung und Unterhaltung der Kompensationsflächen zu diagnostizieren, was deren naturschutzfachliche Wirkung deutlich einschränkt (vgl. etwa LUWG ${ }^{19} 2005$ am Beispiel Rheinland-Pfalz).

Es ergibt sich daher die Notwendigkeit zur Weiterentwicklung des Kompensationsflächenmanagements, um einerseits bestehende Umsetzungsdefizite abzubauen und andererseits flächensparende und innovative Kompensationsmaßnahmen verstärkt zu etablieren. Hier sind neben Aufwertungsmaßnahmen bestehender Ökosysteme vor allem Entsiegelungsmaßnahmen und produktionsintegrierte Kompensationsmaßnahmen ${ }^{20} \mathrm{zu}$ nennen. Insbesondere Letztere sind grundsätzlich dazu geeignet, eine naturschutzfachlich hochwertige Kompensation zu gewährleisten, gleichzeitig aber die Fortführung der landwirtschaftlichen Nutzung zu ermöglichen. Dies trägt zur Akzeptanzsteigerung entsprechender Maßnahmen bei und sichert deren langfristige Unterhaltung (vgl. Czybulka et al. 2012). Zur Umsetzung des Kompensationsflächenmanagements bedarf es innovativer Kompensationsflächenkataster und der Stärkung entsprechender Institutionen zur Planung, Koordinierung und Umsetzung der Maßnahmen. Um einen bundeseinheitlichen rechtlichen Rahmen zu schaffen und mit einheitlichen Bewertungsverfahren auch länderübergreifende Kompensationen zu ermöglichen, bietet sich die Verabschiedung einer bundeseinheitlichen Kompensationsverordnung an, um einer fortschreitenden Rechtszersplitterung durch weitere Länderverordnungen vorzubeugen. ${ }^{21}$

\section{Weiterentwicklung der Gemeinsamen Agrarpolitik}

Die EU-Agrarpolitik ist eines der zentralen Steuerungs- und Anreizinstrumente der Landnutzung, wobei nicht nur die Land- und Forstwirtschaft gefördert wird, sondern auch die wirtschaftliche Entwicklung in ländlichen Gebieten sowie lokale Dorfentwicklungsprozesse adressiert sind. Sie setzt dabei in der Förderperiode ab 2014 nach wie vor den größten Teil der Finanzmittel für entkoppelte, flächengebundene Direktzahlungen an die Landwirtschaft ein (erste Säule), die eine nur geringe Steuerungswirkung entfalten.

\footnotetext{
${ }^{19}$ Landesamt für Umwelt, Wasserwirtschaft und Gewerbeaufsicht Rheinland-Pfalz.

${ }^{20}$ Über produktionsintegrierte Kompensationsmaßnahmen in der Landwirtschaft wird mit Hilfe zeitlich befristeter und genau definierter Leistungen des bewirtschaftenden Landwirts eine Aufwertung für den biotischen oder abiotischen Ressourcenschutz angestrebt, ohne die Fläche der landwirtschaftlichen Nutzung zu entziehen.

${ }^{21}$ Der aktuell zweite Entwurf der Bundes-Kompensationsverordnung stellt in diesem Zusammenhang einen deutlichen Rückschritt gegenüber dem ursprünglichen Entwurf dar. Zudem ist aktuell ungewiss, ob generell eine Ländermehrheit zur Verabschiedung einer Bundesverordnung erreicht werden wird.
} 
Perspektivisch erscheint daher die Beibehaltung der Flächenprämien im Rahmen der GAP nicht zielführend und auch nicht notwendig, da die Wettbewerbsfähigkeit der europäischen Landwirtschaft auch bei einem schrittweisen Abbau der Direktzahlungen nicht gefährdet wäre (vgl. Isermeyer 2012, S. 58). In Regionen, in denen dies nicht der Fall wäre, stünden dann aufzustockende Mittel der heutigen zweiten Säule in Form von Ausgleichszulagen für benachteiligte Regionen zur Verfügung (vgl. WBA 2010, S. 28). Erfolg versprechender und gesellschaftlich akzeptabler wäre der zielgerichtete Mitteleinsatz für Instrumente der zweiten Säule der GAP, die dann nicht mehr wie bisher von den Mitgliedstaaten kofinanziert werden müssten, sondern ähnlich wie die aktuellen Flächenprämien zu $100 \%$ aus dem EU-Haushalt finanziert werden könnten. ${ }^{22}$ Da viele Herausforderungen im Agrarbereich jedoch auch über die derzeitige zweite Säule nicht zu bewältigen sind, wird neben dem schrittweisen Abbau der ersten und der finanziellen Aufstockung der zweiten Säule der GAP eine Aufteilung der zweiten Säule in eine ländliche Entwicklungspolitik (dezentral betrieben, sektorübergreifend konzipiert) sowie eine Agrarstrukturpolitik diskutiert (vgl. Isermeyer 2014, S. 11 f.; Weingarten 2014).

\section{Vermehrter Einsatz informeller Planungs- und Handlungsansätze im Klimawandel}

Die Rolle informeller Planungs- und Handlungsansätze wurde bereits in Zusammenhang mit der Raum- und Umweltplanung angesprochen. Auch die Instrumente der zweiten Säule der GAP weisen großes Potenzial zur Bearbeitung von Themen der Klimaanpassung und des Klimaschutzes auf.

Eine der drei Prioritäten der Förderung der ländlichen Entwicklung im Rahmen der GAP ist die Gewährleistung der nachhaltigen Bewirtschaftung der natürlichen Ressourcen und des Klimaschutzes. Hierzu werden neben Agrarumwelt- und Klimamaßnahmen auch regionale Entwicklungsprozesse in ländlichen Räumen gefördert. In Deutschland erfolgt die Förderung solcher Prozesse über die Integrierte Ländliche Entwicklung (ILE) ${ }^{23}$ sowie das europaweit bedeutsame Strukturförderprogramm $\mathrm{LEADER}^{24}$. Informelle Instrumente der ländlichen Regionalentwicklung könnten und sollten künftig stärker als bisher Belange des Klimaschutzes und der Klimaanpassung aufgreifen. Frelih-Larsen et al. (2014, S. 50 ff.) stellen für das Beispiel LEADER zwar fest, dass in der Förderperiode 2007 bis 2013 klimawandelbezogene Themen nach wie vor deutlich hinter klassischen Themen ländlicher Entwicklung zurückstanden, identifizieren jedoch auch ein deutliches Potenzial dieses

\footnotetext{
${ }^{22} \mathrm{Vgl}$. hierzu beispielhaft die gleichlautenden Empfehlungen des Thünen-Instituts (Isermeyer 2012; Isermeyer et al. 2014), des Bioökonomierates (Birner et al. 2014) sowie des Bundesamtes für Naturschutz (vgl. Oppermann et al. 2013, S. 192).

${ }^{23}$ Zum Beispiel über Integrierte ländliche Entwicklungskonzepte (ILEK), Pläne für die Entwicklung von Gemeinden sowie Dorferneuerung und -entwicklung.

${ }^{24}$ LEADER ist ein Förderprogramm der Europäischen Union zur Entwicklung des ländlichen Raumes. Ziel der Förderung ist die Unterstützung einer eigenständigen und nachhaltigen Regionalentwicklung in ländlichen Gebieten, gefördert über den europäischen Landwirtschaftsfonds ELER.
} 
Instrumentariums in diesem Kontext. Um ein vermehrtes Aufgreifen entsprechender Projekte in den Regionen zu erreichen, wird es darauf ankommen, die vielfältigen Synergieeffekte von Klimaschutz- und Klimaanpassungsprojekten besser zu kommunizieren, den lokalen Aktionsgruppen methodische Hilfestellungen zur erfolgreichen Umsetzung von Klimaschutzprojekten anzubieten sowie über die Programmgestaltung der einzelnen Bundesländer Klimaschutz und Klimaanpassung prominenter zu verankern (vgl. - zu Hemmnissen und Handlungsoptionen bei der Integration von Klimaschutz und Klimaanpassung in die ländliche Regionalentwicklung - Neubert 2014).

Von besonderem Interesse für den Sektor Landwirtschaft ist die neu geschaffene Europäische Innovationspartnerschaft „Landwirtschaftliche Produktivität und Nachhaltigkeit“ (EIP Agri). Diese soll eine bessere Verknüpfung der Forschung mit der landwirtschaftlichen Praxis sowie einen breiteren Einsatz verfügbarer Innovationsmaßnahmen gewährleisten. EIP Agri eröffnet das Potenzial, über kooperative Handlungsansätze eine wettbewerbsfähige und nachhaltige Landwirtschaft zu erhalten und weiterzuentwickeln und dabei beispielsweise Belange des Moorschutzes, innovative Düngeverfahren oder auch den Anbau alternativer Energiepflanzen voranzubringen. ${ }^{25}$

\section{Stärkung des integrierten Flächenmanagements als Teil einer vorsorgenden Flächenhaushaltspolitik}

Maßnahmenübergreifend bestätigt sich die hohe Bedeutung eines integrierten Flächenmanagements. Zahlreiche dem Klimaschutz und der Klimaanpassung dienende Maßnahmen bedürfen des Landerwerbs und der Bodenbevorratung sowie der Betreuung und Durchführung geeigneter Maßnahmen der Flurbereinigung und ländlichen Bodenordnung. Beispielhaft sei an dieser Stelle auf großflächige Moorrenaturierungen, den vorbeugenden Hochwasserschutz durch Deichrückverlegungen sowie das Kompensationsflächenmanagement verwiesen. Institutionen und Einrichtungen, die Aufgaben eines integrierten Flächenmanagements übernehmen, Landnutzungskonflikte entschärfen und einen Ausgleich unterschiedlicher Nutzungsansprüche befördern, gilt es zu stärken und mit ausreichenden Ressourcen auszustatten.

\section{Etablierung einer integrierten Umweltbeobachtung}

Die Entwicklung und rechtliche Begründung von Strategien und Maßnahmen zum Umgang mit den Folgen des Klimawandels bedürfen einer hinreichend belastbaren und aktuellen Informationsgrundlage zur Entwicklung der Umwelt sowie der zu erwartenden klimatischen Veränderungen und deren Folgewirkungen. Insofern kommt einer integrierten und schutzgutübergreifenden Umweltbeobachtung eine zentrale Bedeutung zu. Nur so können Wirkungszusammenhänge hergeleitet und Projektionen und Prognosen für die zu erwartenden regionalen Klimafolgenentwicklungen erstellt werden. Aktuell herrscht jedoch z. T. die unkoordinierte Betrachtung einzelner Umweltmedien

\footnotetext{
${ }^{25}$ Weitere Informationen unter www.netzwerk-laendlicher-raum.de/themen/eip-agri.
} 
vor $^{26}$, ohne dass eine bundeseinheitliche Datenerhebung, -auswertung und -bearbeitung stattfindet (vgl. auch Möckel und Reese 2010, S. 406). Notwendig sind jedoch - wo sinnvoll und zielführend - eine bessere Integration, Systematisierung und Harmonisierung der bestehenden sektoralen bzw. medialen Beobachtungs- und Monitoringprogramme sowie eine integrative und ökosystemare Datenauswertung. Koordinierungsbedarf besteht dabei zwischen Bund, Ländern und Kommunen ebenso wie zwischen den einzelnen Sektoren und Fachplanungen.

\section{Netzwerk Vulnerabilität}

Im Kontext der Deutschen Anpassungsstrategie an den Klimawandel (DAS) wurde als eine Maßnahme des Aktionsplans ein Behördennetzwerk Vulnerabilitätsbetrachtung definiert (Bundesregierung 2011). Dieses Netzwerk von 16 Behörden und Institutionen aus neun Ressorts zielt darauf übergreifend die Verwundbarkeit Deutschlands gegenüber dem Klimawandel als Grundlage für eine Priorisierung der Klimarisiken für Deutschland zusammenzutragen. Die inter- und transdisziplinäre Kooperation zwischen allen Beteiligten des Netzwerkes Vulnerabilität erlaubt das bestehende Wissen zusammenzuführen und ein gemeinsames Verständnis bezüglich der Risiken des Klimawandels sowie der Vulnerabilität der verschiedenen Sektoren und Regionen zu entwickeln. Es wurden vierzehn sektorale Handlungsfelder identifiziert Bauwesen, Biologische Vielfalt, Boden, Energiewirtschaft, Finanzwirtschaft, Fischerei, Industrie und Gewerbe, Küsten- und Meeresschutz, Landwirtschaft, menschliche Gesundheit, Tourismuswirtschaft, Verkehr und -infrastruktur, Wald- und Forstwirtschaft sowie Wasserhaushalt und -wirtschaft. Als Querschnittthemen wurden die Themenbereiche Raumordnung, Regional- und Bauleitplandung sowie Bevölkerungs- und Katastrophenschutz benannt (adelphi/PRC/EURAC 2015).

\subsubsection{Institutionelle Gestaltungsoptionen im Siedlungswesen}

Im Sektor Siedlung und Verkehr liegt der Fokus auf Maßnahmen zur Reduzierung der Flächeninanspruchnahme, insbesondere durch eine forcierte Innenentwicklung, die Ausschöpfung baulicher Dichten im Neubau, den „Rückzug aus der Fläche“, einen restriktiveren Freiraumschutz, die Reduktion der Flächeninanspruchnahme für Verkehrsinfrastruktur sowie die Eindämmung des Individualverkehrs durch geeignete Maßnahmen wie z. B. die Stärkung des ÖPNV. Mit Blick auf die Notwendigkeit zur Klimaanpassung im Siedlungssektor befassen sich weitere Maßnahmen mit einem verbesserten Hochwasserschutz sowie dem Erhalt und der Entwicklung innerstädtischer Freiflächen.

\footnotetext{
${ }^{26}$ Zum Beispiel FFH-Monitoring, Monitoring Luftqualität und Lärmbelastung, Gewässerqualität nach Wasserrahmenrichtlinie, Bundeswaldinventur etc.
} 


\subsubsection{Beiträge zum Klimaschutz durch die Reduzierung der Flächeninanspruchnahme}

Die Reduzierung der Flächeninanspruchnahme ist als gesellschaftliches Ziel gesetzt und hat in den vergangenen Jahren - getragen durch die politische Willensbekundung der Nachhaltigkeitsstrategie der Bundesregierung - umfänglich Eingang gefunden in das Raumordnungs- und Bauplanungsrecht sowie ergänzendes Fachrecht. Zur Umsetzung steht ein breites Spektrum formal-rechtlicher und informeller Instrumente zur Verfügung, die in vielen Regionen und Kommunen erfolgreich zur Umsetzung kommen. Gespeist wird die Planungspraxis dabei aus vielfältigen innovativen Handlungsansätzen, die aus verschiedensten Forschungsprojekten und Modellvorhaben hervorgegangen sind. Gleichwohl wird das 30-ha-Ziel bis 2020 nicht erreicht werden (vgl. Abschn. 4.4), da die zur Verfügung stehenden Instrumente in den einzelnen Bundesländern bisher nicht flächendeckend und hinreichend konsequent zum Einsatz kommen und eine nachhaltige Flächenpolitik in vielen Kommunen kein prioritäres Ziel darstellt (vgl. Einig 2011).

Wesentliche Beiträge zur Reduzierung der Flächeninanspruchnahme könnte vor allem die Raumordnung leisten, insbesondere durch den verstärkten Einsatz restriktiver, flächeverknappender Ansätze in der Landes- und Regionalplanung ${ }^{27}$, die jedoch vielfach als zu starker Eingriff in die kommunale Selbstverwaltung angesehen und daher nur zögerlich angewendet werden. Zudem erscheinen verstärkte Anstrengungen für innovative und integrativ arbeitende regionale Kooperationen ${ }^{28}$ notwendig, da nur auf dieser Ebene Fragen einer nachhaltigen und regional abgestimmten Flächenhaushaltspolitik zielgerichtet und kooperativ gelöst werden können. Perspektivisch steht die Ergänzung des Instrumentenkastens um weitere anreiztechnische Instrumente wie beispielsweise handelbare Flächenzertifikate ${ }^{29}$ zur Diskussion. Hierzu besteht jedoch weiterer Forschungsbedarf. Zudem ist im Falle einer Implementierung eine instrumentelle Überfrachtung des Planungssystems zu vermeiden. Entlang der einzel-

\footnotetext{
${ }^{27}$ Negativ-restriktiver Freiraumschutz (z. B. Vorranggebiete für Natur und Landschaft), ergänzt durch positiv-planerische Instrumente (z. B. Dichtewerte für Wohnbauflächen, positiv-allokative Siedlungssteuerung durch Vorranggebiete, Vorgaben zur Innenentwicklung, Vorgaben zur Eigenentwicklung; vgl. vertiefend BMVBS 2012).

${ }^{28}$ Beispielhafter Verweis auf den Regionalverband Ruhr, der als Regionalplanungsbehörde auch regionale Wirtschafts- und Tourismusförderung sowie die Öffentlichkeitsarbeit für das Gebiet der Metropole Ruhr wahrnimmt.

${ }^{29}$ Seit geraumer Zeit wird die Einführung handelbarer Flächenzertifikate in Anlehnung an den Emissions-Zertifikatehandel diskutiert und von F+E-Vorhaben begleitet, z. B. „Planspiel Flächenhandel“" im Auftrag des Umweltbundesamtes (Laufzeit 10/2012 bis 12/2015; www. flaechenhandel.de). Grundidee des Konzeptes ist es, dass Kommunen für die Baulandausweisung im Außenbereich eine entsprechende Menge an Zertifikaten aufbringen müssen. Diese sind zwischen den Kommunen frei handelbar. Weist eine Kommune kein Bauland aus, kann es die ihm zugeteilten ungenutzten Zertifikate an andere Kommunen veräußern, die mehr Zertifikate benötigen, als ihnen zugeteilt wurden.
} 
nen modellierten Maßnahmen, die zur Reduzierung der Flächeninanspruchnahme beitragen können, werden im Folgenden konkrete Handlungsoptionen aufgezeigt, die einer weiteren Maßnahmenbeförderung zuträglich sein können.

\section{Stärkung der Innenentwicklung}

Für eine konsequentere Lenkung der Siedlungsflächenentwicklung auf den Innenbereich im Sinne der Innenentwicklung steht ein umfängliches Instrumentarium zur Verfügung, das durch die kommunalen Planungsträger jedoch oftmals nicht voll ausgeschöpft wird. Hinzu treten Grenzen der Innenentwicklung, die den Beitrag dieser planerischen Handlungsmaxime zur Erreichung der Flächensparziele der Bundesregierung einschränken. Diese Hemmnisse umfassen insbesondere

- die schwierige Aktivierbarkeit von Innenentwicklungspotenzialen,

- die regionale Persistenz der Wachstumsorientierung in der kommunalen Baulandpolitik,

- die der Innenentwicklung entgegenstehenden Präferenzen der Wohnraumnachfrager,

- räumliche Divergenzen zwischen vorhandenen Innenentwicklungspotenzialen und der Baulandnachfrage,

- die oftmals unzureichenden finanziellen und personellen Kapazitäten der Kommunen sowie

- städtebauliche, ökologische und immissionsschutzrechtliche Grenzen der Innenentwicklung.

Für eine weitere Beförderung der Innenentwicklung bedarf es neben der konsequenten Anwendung der Bodenschutzklausel des BauGB vor allem verstärkter Bemühungen zur weitergehenden Etablierung kommunaler und regionaler Flächenmanagementstrategien. In diesem Zusammenhang relevante informatorische Instrumente, wie beispielsweise regionale und kommunale Innenentwicklungskataster, sind konsequenter als bisher zu erarbeiten. Zudem erscheint die Einführung obligatorischer InfrastrukturfolgekostenBerechnungen als verpflichtender Bestandteil planerischer Entscheidungen zielführend, was durch eine entsprechende Modifikation der Bodenschutzklausel des BauGB möglich wäre.

Zur Überwindung der regional nach wie vor persistenten angebotsorientierten Baulandpolitik vieler Kommunen ist der verstärkte Einsatz interkommunal abgestimmter Baulandstrategien angezeigt, deren Erarbeitung durch informelle Handlungs- und Planungsansätze eingeleitet werden kann. Wichtige Impulse können durch eine intensivierte Öffentlichkeitsarbeit und Sensibilisierung zum Thema Innenentwicklung auf regionaler und kommunaler Ebene ergehen. Flankierend bedarf es dringend der weitergehenden Befähigung der Kommunen zur Umsetzung von Innenentwicklungsstrategien sowie des Abbaus hemmender Faktoren und instrumenteller Schwächen. Hier ist vor allem auf die notwendige Stärkung bzw. konsequente Anwendung des städtebaulichen Instrumentariums für einen effektiveren Flächen- und Gebäudezugriff zur 
Aktivierung von Innenentwicklungspotenzialen hinzuweisen. ${ }^{30}$ Zusätzlich ist die Überprüfung lärmschutzrechtlicher Regelungen im Zusammenspiel mit dem Städtebaurecht angezeigt, da diese häufig ein Hemmnis für Innenentwicklungsvorhaben darstellen können (vgl. Bunzel et al. 2014, S. 10).

Notwendig ist zudem eine verstärkte Ausrichtung des vorhandenen Förderinstrumentariums auf die Innenentwicklung sowie die Sicherstellung einer ausreichenden personellen und finanziellen Mittelausstattung der Kommunen, um den Mehraufwand für die erweiterten Ermittlungsaufgaben und die Umsetzung von Aktivierungskonzepten auszugleichen. Perspektivisch ergibt sich die Notwendigkeit der Berücksichtigung flächenbezogener Komponenten im kommunalen Finanzausgleich, der bisher zu sehr durch die Einwohnerorientierung geprägt ist und so flächenpolitische Fehlanreize induziert.

\section{Ausschöpfung baulicher Dichte im Neubau}

Auf die vermehrte Ausschöpfung baulicher Dichte im Neubau können die Kommunen direkten Einfluss nehmen, indem sie das zur Verfügung stehende Instrumentarium der Bauleitplanung ausnutzen und eine flächensparende Siedlungsentwicklung umsetzen, insbesondere durch die Festsetzung von Mindestdichten des Maßes der baulichen Nutzung nach Baunutzungsverordnung (BauNVO). Eine solche flächensparende Siedlungsentwicklung kann durch einen kommunalen Baulandbeschluss nach außen kommuniziert und als Selbstbindung zum Flächensparen verstanden werden. In den Teilräumen, in denen auf kommunaler Ebene keine hinreichende Ausschöpfung der baulichen Dichten erfolgt, kann auf übergeordneter Ebene die Raumordnung Einfluss auf das kommunale Planungsgeschehen nehmen, indem flächeverknappende Instrumente der Landes- und Regionalplanung zum Einsatz kommen (s. o.) und vermehrt Dichtewerte für Wohnbauflächen durch die Regionalplanung vorgegeben werden. ${ }^{31}$ Weitere Handlungsoptionen ergeben sich durch die verstärkte Einbeziehung von Infrastrukturfolgekosten-Berechnungen in planerische Entscheidungen (s. o.), die weitere Etablierung planerisch-architektonischer Lösungen flächensparenden Bauens sowie die informatorische Beeinflussung der Wohnraumnachfrage, hin zu flächensparenden und gleichzeitig den Ansprüchen modernen Wohnens genügenden baulichen Lösungen.

\footnotetext{
${ }^{30}$ Beispielsweise durch einen zielgerichteten Einsatz gemeindlicher Vorkaufsrechte, den vermehrten Einsatz von Entwicklungs- und Sanierungsmaßnahmen sowie die Schärfung des Wohnungsaufsichtsrechts für einen zielgerichteteren Umgang mit verwahrlosten Immobilien.

${ }^{31}$ So findet sich beispielsweise im Regionalplan der Region Stuttgart (vgl. Verband Region Stuttgart 2009, S. 56) ein raumordnerisches Ziel zur Freiraumsicherung/Bruttowohndichte. Dieses besagt, dass zur Reduzierung der Belastung von Freiräumen durch neue Siedlungsflächeninanspruchnahme bei allen Neubebauungen eine angemessene Bruttowohndichte einzuhalten ist, die entlang der zentralörtlichen Stufen variiert. Hinzuweisen ist an dieser Stelle auf die Tatsache, dass die Ausweisung von Mengenzielen häufig zu Konflikten mit dem Bestimmtheitserfordernis in der Raumordnung führt.
} 


\section{Rückzug aus der Fläche (dezentrale Konzentration)}

Mit einem „Rückzug aus der Fläche“ können durch eine raumordnerisch gesteuerte Siedlungsentwicklung im Sinne der dezentralen Konzentration und die Ausschöpfung von Rückbaupotenzialen sowie schrumpfungsbedingter Siedlungsdegressionen Beiträge zum Klimaschutz geleistet werden. Für eine restriktivere Umsetzung des Konzeptes der dezentralen Konzentration durch die Lenkung der Siedlungs- und Verkehrsflächenentwicklung auf zentrale Orte bedarf es differenzierter Betrachtungsweisen in Abhängigkeit der jeweiligen regionalen raumstrukturellen Ausgangssituation und den zu erwartenden demographischen Entwicklungen. Darauf aufbauend ist in erster Linie die Anpassung und Weiterentwicklung raumordnerischer Steuerungsansätze (z. B. durch eine restriktivere Steuerung der Eigenentwicklung) anzustreben sowie eine Weiterentwicklung des zentralörtlichen Systems als Grundgerüst zur Steuerung der Raum- und Siedlungsstruktur. Insbesondere kommt es auf eine Straffung des Systems, die ortsteilscharfe Ausweisung der zentralörtlichen Funktionen sowie die Förderung zentralörtlicher Kooperationsformen an. Von Relevanz ist zudem die Förderung des regionalen Diskurses bei der Weiterentwicklung des Instrumentariums (Akzeptanzschaffung und damit erhöhte Effektivität) sowie die Bereitstellung ausreichender finanzieller und personeller Ressourcen auf Ebene der Landes- und Regionalplanung, die zur Umsetzung einer aufwändigen Standortsteuerung notwendig sind.

Hinsichtlich eines möglichen Rückbaus ländlich-peripherer Siedlungen mit Schrumpfungstendenzen ist zunächst auf den weiteren Forschungsbedarf zur besseren Erschließung der Thematik hinzuweisen. Hierzu ist die Bereitstellung von Fördermitteln für innovative Pilotprojekte und Erprobungsvorhaben sowie die Fortführung der Neuinterpretation der Gleichwertigkeit der Lebensverhältnisse im Sinne des § 1 (2) ROG erforderlich. Unabhängig davon erscheint es unabdingbar, eine aktive Begleitung der ablaufenden Schrumpfungsprozesse anstelle eines passiven Zulassens zu gewährleisten. Hierzu kommt es nach Küpper et al. (2013) vor allem darauf an,

- Dorferneuerungs- und/oder Städtebaufördermittel verstärkt an die Erarbeitung und Fortschreibung regionaler integrierter Siedlungsentwicklungskonzepte zu knüpfen,

- analog zum Stadtumbau in schrumpfenden ländlichen Räumen einen regionalen Siedlungsumbau zu etablieren,

- neuere gesellschaftliche Trends (Energiewende, regionale Produkte) als Chance für schrumpfende Regionen zu erkennen,

- öffentliche Investitionen in Infrastruktur und Einrichtungen der Daseinsvorsorge stärker als bisher dahingehend zu überprüfen, ob sie langfristig tragfähig sind,

- Regionaletats für einen effizienten Fördermitteleinsatz einzuführen und im Sinne des ,zulassenden Staates“ rechtliche Standards zu lockern, um regional angepasste und innovative Lösungen von Grundversorgungsleistungen zu ermöglichen. 
Sollte perspektivisch eine aktive Rückbaupolitik für Teilräume oder einzelne Ortslagen in Frage kommen, ergäbe sich die Notwendigkeit, neue planerische, ökonomische und informelle Instrumente $\mathrm{zu}$ implementieren. ${ }^{32}$ Weitergehende Erkenntnisse können perspektivisch aus der MORO-Vorstudie des BBSR als Vorbereitung eines Planspiels zur „Anpassung peripherer Siedlungsstrukturen“" hervorgehen.

\section{Restriktiverer Freiraumschutz}

Ein restriktiverer Freiraumschutz kann ebenfalls zur effektiveren Steuerung der Flächeninanspruchnahme beitragen. Um die standortsteuernde Wirkung von Schutzgebietsausweisungen zu erhöhen, bedarf es weniger der Änderung des bestehenden Naturschutzrechtes als vielmehr der zielgerichteten Ausgestaltung der Schutzgebietsverordnungen. Dies betrifft insbesondere Landschaftsschutzgebiete, die häufig in Siedlungsrandbereichen liegen und in denen flächenrelevante Zielsetzungen vermehrt aufgenommen werden könnten. Eine Stärkung der Regionalplanung zum Schutz von Natur und Landschaft kann durch die prioritäre Ausweisung von Vorrang- gegenüber Vorbehaltsgebieten erreicht werden. Dabei ist jedoch abzuwägen zwischen der höheren Bindungswirkung eines Vorranggebietes auf der einen und der höheren Akzeptanz und Flexibilität eines Vorbehaltsgebietes auf der anderen Seite. Für die Ausweisung von Pufferflächen rund um Naturschutzgebiete und Nationalparks kommen in erster Linie Landschaftsschutzgebiete in Frage. Deren tatsächliche Schutzwirkung hängt von der konkreten Ausgestaltung der einzelnen Schutzgebietsverordnungen sowie allgemein von der konsequenten Ausgestaltung und Akzeptanz der Kategorie „Landschaftsschutz“ ab.

\section{Reduktion der Flächeninanspruchnahme durch Verkehr}

Soll eine Reduktion der Flächeninanspruchnahme durch Verkehr realisiert werden, rückt zunächst der Verzicht auf neue Trassenplanungen in den Fokus. In diesem Zusammenhang ist vor allem der Bundesverkehrswegeplan (BVWP) von Relevanz, da bereits auf dieser Ebene wichtige Weichenstellungen für die Planung der Bundesschienenwege und Bundesfernstraßen getroffen werden. Für den BVWP 2015 musste erstmals eine Strategische Umweltprüfung (SUP) nach Umweltverträglichkeitsprüfungsgesetz (UVPG) durchgeführt werden. ${ }^{33}$ Die Durchführung einer SUP für den

\footnotetext{
${ }^{32}$ Aktuelle Fachdiskurse (z. B. Berlin-Institut für Bevölkerung und Entwicklung 2011) befassen sich in diesem Zusammenhang mit Überlegungen zur Umsetzung einer aktiveren Siedlungspolitik und der gezielten Aufgabe einzelner Dörfer, der raumordnerischen Identifizierung und Festlegung von Rückzugsräumen, der Etablierung von Rückbau-Fonds sowie der Entwicklung von Umzugsmanagements für ausgewählte Rückzugsräume.

${ }^{33}$ Bisher wurden Umweltbelange lediglich über die Monetarisierung bestimmter Umweltkriterien im Rahmen der Nutzen-Kosten-Analyse (NKA) berücksichtigt und die Umweltrisikoeinschätzung (URE) mit integrierter FFH-Verträglichkeitseinschätzung.
} 
BVWP ist zu begrüßen und kann dazu beigetragen, die Umweltfolgen durch vorsorgliche Umplanungen und einschränkende Nebenbestimmungen zu begrenzen, sofern Aspekte des Flächenverbrauchs hinreichend Berücksichtigung finden. Letztlich bleibt die Ausweisung neuer Trassen jedoch ein politischer Abwägungsprozess, in dessen Rahmen klima- und umweltpolitische Fragestellungen häufig unterliegen. Gleiches gilt für Raumordnungsverfahren nach $\S 15$ ROG, über die eine Prüfung der Raumverträglichkeit raumbedeutsamer Planungen und Maßnahmen erfolgt. Insofern obliegt es den politischen Entscheidungsträgern, bei der Entscheidung über den Bundesverkehrswegeplan umwelt- und naturschutzfachlichen Auswirkungen ein besonderes Gewicht beizumessen, um unnötige Flächeninanspruchnahmen zu vermeiden. Dies entspräche der Umsetzung der Grundkonzeption für den BVWP 2015 (vgl. BMVI 2014), die unter Punkt 1 eindeutig Erhaltungsinvestitionen gegenüber Aus- und Neubauvorhaben priorisiert.

Damit einher geht die notwendige Reduktion des innerstädtischen Flächenbedarfs für Verkehrsflächen entsprechend des städtebaulichen Leitbildes der Stadt der kurzen Wege. Anzustreben ist eine flächensparende Siedlungsentwicklung, wobei sich Anknüpfungspunkte an die Ausschöpfung der baulichen Dichte im Neubau sowie die Ausführungen zur Innenentwicklung ergeben. Auch die im nachfolgenden Abschnitt angesprochene Beförderung des ÖPNV wäre der Maßnahme zuträglich, da der Flächenbedarf pro Person und Verkehrsmittel im ÖPNV deutlich geringer ausfällt als im motorisierten Individualverkehr. ${ }^{34}$

Gleichzeitig bestehen Potenziale hinsichtlich des Rückbaus nicht mehr benötigter Infrastrukturen. Zielgerichtete Rückbauprozesse könnten durch Anreizelemente der aktuell diskutierten Zertifikate basierten Flächenhandels über sogenannte „Weiße Zertifikate" befördert werden. Alternativ wäre eine Anreizsteuerung über entsprechend aufzulegende bzw. auszubauende Förderprogramme in der Städtebauförderung bzw. der Förderung der Entwicklung ländlicher Räume möglich.

\section{Stärkung des öffentlichen Personennahverkehrs}

Um eine Verringerung des motorisierten Individualverkehrs und somit einen verringerten Flächenbedarf für Verkehrsinfrastrukturen sowie eine Reduzierung von THG-Emissionen zu erreichen, bedarf es der weiteren Stärkung des öffentlichen Personennahverkehrs (ÖPNV). Hierzu ist insgesamt eine deutliche Attraktivitätssteigerung anzustreben, wie sie vielerorts bereits realisiert wird. Ein wesentliches Hemmnis für die weitere Stärkung des ÖPNV stellt die Finanzierung dar. Ein eigenes ÖPNV-Finanzierungsgesetz oder die Einbeziehung der Aufgaben des Gemeindeverkehrsfinanzierungsgesetzes in das Regionalisierungsgesetz könnten eine konsistente und verfassungsgerechte Finanzierung darstellen. In diesem Kontext sind weitere Über-

\footnotetext{
${ }^{34}$ Basierend auf Berechnungen der Stadt Zürich, Tiefbauamt, Mobilität + Verkehr, 2011, vgl. Walter (2013, S. 6).
} 
legungen zur Beitragsfinanzierung des ÖPNV anzustellen. ${ }^{35}$ Unabhängig der drängenden Finanzierungsfragen wird es künftig vor allem darauf ankommen, eine nachhaltige Verkehrsentwicklungsplanung zu etablieren, eingebettet in das vertikale und horizontale Planungsgeflecht. ${ }^{36}$ Wichtige Beiträge zur Stärkung des ÖPNV können zudem von informellen Handlungs- und Planungsansätzen ausgehen. So werden zum Beispiel im Rahmen des sogenannten Mobilitätsmanagements Ansätze zur Beeinflussung der Verkehrsnachfrage entwickelt, mit dem Ziel, den Personenverkehr effizienter, umwelt- und sozialverträglicher und damit nachhaltiger zu gestalten. Dabei kommen in erster Linie informatorische, kommunikative, motivierende und koordinierende Instrumente zum Einsatz, um das Verkehrsverhalten der Bevölkerung in Richtung umweltfreundlicher Verkehrsmittel zu beeinflussen und insbesondere den Anteil des motorisierten Individualverkehrs zu senken. Weiterhin könnten in Abhängigkeit der lokalen Gegebenheiten weitere Lenkungsinstrumente mit einbezogen werden, etwa Umweltzonen oder auch ökonomische Anreizinstrumente wie das Road-Pricing und die City-Maut.

\subsubsection{Ausgewählte Maßnahmen zur Klimaanpassung im Sektor Siedlung und Verkehr}

Zusätzlich zu den Beiträgen des Siedlungssektors zum Klimaschutz (Reduzierung der Flächeninanspruchnahme) besteht Handlungsbedarf zur Anpassung der Siedlungs- und Verkehrsstrukturen an die Folgen des Klimawandels. Hierzu wird exemplarisch auf die Stärkung des Hochwasserschutzes sowie den Erhalt und die Entwicklung innerstädtischer Freiflächen eingegangen.

\section{Stärkung des Hochwasserschutzes}

Für eine Stärkung des Hochwasserschutzes in der räumlichen Planung bedarf es zuallererst einer konsequenteren Anwendung des zur Verfügung stehenden Instrumentariums entlang der Handlungsempfehlungen von ARGEBAU, LAWA und MKRO (2010) sowie einer konsequenten Auslegung der Vorschriften des $§ 78$ Wasserhaushaltsgesetz (WHG) zur Siedlungsentwicklung in festgesetzten Überschwemmungsgebieten. Darüber hinaus sind neue und über die gängige Planungspraxis hinausgehende planerische Ansätze zu prüfen, insbesondere zur stärkeren Berücksichtigung extremer Hochwasserereignisse. Dies wäre beispielsweise möglich durch

\footnotetext{
${ }^{35}$ Insbesondere Drittnutzer (Arbeitgeber, Einzelhändler mit Kundenverkehr etc.) könnten in die Finanzierung mit einbezogen werden (vgl. Bracher et al. 2014).

${ }^{36}$ Hierzu bedarf es v. a. einer validen Datengrundlage über die Verkehrs-, Bevölkerungs- und Raumentwicklung, eines an Nachhaltigkeitsaspekten orientierten Zielsystems, einer sachgerechten Akteursbeteiligung sowie der Einbindung der Verkehrsentwicklungsplanung in regionale Kontexte (vgl. Wolfram et al. 2010).
} 
- die Einbeziehung besiedelter Gebiete in ausgewiesene Vorranggebiete (zur Steuerung der Bautätigkeit im unbeplanten Innenbereich) sowie

- die Ausweisung von Vorranggebieten für Extremhochwassergebiete $\left(\mathrm{HQ}_{\text {extrem }}\right)$ (Ermöglichung von Vorgaben zum hochwasserangepassten Bauen; vgl. Seifert 2012). ${ }^{37}$

Bei der Planung technischer Hochwasserschutzanlagen wird in der Regel ein Hochwasserereignis zugrunde gelegt, das statistisch gesehen alle 100 Jahre auftritt $\left(\mathrm{HQ}_{100}\right)$. Um den Auswirkungen des Klimawandels Rechnung zu tragen, erscheint die Anrechnung von Klimaänderungsfaktoren auf diesen vergangenheitsbezogenen Wert geboten, um der künftig erhöhten Eintrittswahrscheinlichkeit extremer Hochwasserereignisse gerecht zu werden. Gleichwohl muss der Fokus weiterhin auf dem vorbeugenden Hochwasserschutz liegen (Auenrenaturierung, Stärkung des natürlichen Wasserrückhalts etc.), da massive Investitionen in den technischen Hochwasserschutz langfristig paradoxe Folgen haben können, indem sie durch die Vermittlung eines vermeintlichen Sicherheitsgefühls (vor einem $\mathrm{HQ}_{100}$ ) zu einem Rückgang des Risikobewusstseins sowie der Handlungsbereitschaft und -fähigkeit führen können und so zusätzliche private Vorsorgemaßnahmen hemmen. Damit gehen weitere Notwendigkeiten eines verbesserten Hochwasserrisikomanagements einher, in erster Linie

- die bessere Abstimmung zwischen Hochwasserplanung und räumlicher Gesamtplanung,

- die Forderung und Förderung privater Hochwasservorsorge,

- die Prüfung der Einführung einer Pflichtversicherung für Hochwasserschäden ${ }^{38}$ sowie

- die Erhöhung des Hochwasserbewusstseins in der Bevölkerung.

\section{Erhalt und Entwicklung innerstädtischer Freiflächen}

Der Erhalt und die Entwicklung innerstädtischer Freiflächen bedürfen der planerischen und konzeptionellen Umsetzung einer klimawandelgerechten Freiraumplanung. Hierzu ist eine Erhöhung des städtebaupolitischen Stellenwerts der kommunalen

\footnotetext{
${ }^{37}$ Entsprechende gesetzgeberische Aktivitäten sind in Sachsen bereits erfolgt. Die Neufassung des Sächsischen Wassergesetzes sieht zusätzlich zur Ausweisung von Überschwemmungsgebieten gemäß Wasserhaushaltsgesetz die Ausweisung von überschwemmungsgefährdeten Gebieten vor.

${ }^{38}$ Für die Einführung einer Pflichtversicherung für Hochwasserschäden spricht, dass diese der jetzigen Politik der Ad-hoc-Hilfen die politische und ökonomische Grundlage entziehen würde und der Steuerzahler nicht mehr für die Entschädigung von Hochwasserrisiken aufkommen müsste, sondern - v. a. in Risikogebieten - die Gebäudeeigentümer. Darüber hinaus böte die Versicherungspflicht den Betroffenen einen Rechtsanspruch auf Entschädigung zu bezahlbaren Preisen, statt sie von aktuellen Wahlkampf- oder Konjunkturlagen und medialer Aufmerksamkeit abhängig zu machen (vgl. Kuhlicke et al. 2013; Reese 2011, S. 27).
} 
Freiraumplanung notwendig. Darüber hinaus könnten folgende Maßnahmen dazu beitragen, den Erhalt und die Entwicklung innerstädtischer Freiflächen weiter zu befördern:

- Zielgerichteter Einsatz des vorhandenen planerischen Instrumentariums, insbesondere unter Einbeziehung konzeptioneller Planungen in Form informeller, raumbezogener Fachkonzepte;

- Erarbeitung und Bereitstellung methodischer Hilfestellungen für die Träger der kommunalen Freiraumplanung zur Berücksichtigung des Klimawandels;

- Schaffung relevanter Informationsgrundlagen zu Stadtklima und vorhandenem Grünsystem;

- Berücksichtigung stadtklimatischer Belange bereits im Neubau;

- Ausschöpfung von Freiraumpotenzialen durch Rückbau, Entsiegelung und Konzentration gebauter Strukturen.

Um eine erfolgreiche Umsetzung der Freiraumplanung zu gewährleisten sowie den Erhalt und die Pflege innerstädtischer Freiflächen sicherzustellen, wird es zudem darauf ankommen, vermehrt kooperative Ansätze zum Erhalt und zur Entwicklung innerstädtischer Frei- und Grünflächen zu verfolgen (z. B. in Form von Pflegepatenschaften, vertraglich geregelten Beteiligungen von Investoren) und die Erschließung alternativer Grünflächenpotenziale (z. B. Dach- und Fassadenbegrünung) sowie Elemente der natürlichen Sukzession und der Industrienatur in planerische Konzeptionen mit einzubeziehen. Auch ist die Attraktivität und Multifunktionalität von Freiflächen zu fördern. Darüber hinaus können durch die verstärkte energetische Nutzung von Grünschnitt und Pflegematerial in Abhängigkeit der örtlichen Gegebenheiten deutliche Kostensenkungen in der kommunalen Grünpflege realisiert werden. ${ }^{39}$

\subsubsection{Institutionelle Gestaltungsoptionen bei der landwirtschaftlichen Landnutzung}

Die hinsichtlich ihres THG-Einsparungspotenzials wichtigsten Maßnahmen bei der landwirtschaftlichen Landnutzung befassen sich mit der Nutzung organischer Böden, der Anpassung des Düngemanagements, der Substitution fossiler Energie durch Biomasse sowie der Erhaltung und Schaffung von THG-Senken. Im Gegensatz zu den Sektoren „Forstwirtschaft“" und „Siedlung und Verkehr“ erfolgt hier keine Untergliederung nach Aspekten des Klimaschutzes und der Klimaanpassung, da in der Landwirtschaft keine prospektiven Anpassungsmaßnahmen erfolgen, sondern jährlich u. a. durch Sortenwahl und Betriebsmitteleinsatz eine Anpassung an Veränderungen erfolgen kann.

\footnotetext{
${ }^{39}$ Siehe hierzu etwa ein entsprechendes Pilotprojekt der Stadt Kassel: www.stadt-kassel.de/ aktuelles/meldungen/20654/index_print.html.
} 


\subsubsection{Nutzung organischer Böden}

Intakte Moore genießen im deutschen Naturschutzrecht einen sehr hohen Schutzstatus, der sich durch den gesetzlichen Biotopschutz des BNatSchG sowie die in Anhang $1 \mathrm{Nr} 7$ der FFH-Richtlinie ${ }^{40}$ benannten Moortypen ergibt. Jedoch bedarf es für einen besseren Moorschutz der dauerhaften Sicherung bzw. Erreichung des guten Erhaltungszustands aller noch intakten bzw. naturnahen Hoch- und Niedermoore durch eine konsequente Anwendung und Kontrolle nach BNatSchG sowie einer Ausweitung des gesetzlichen Biotopschutzes ( $\$ 30$ BNatSchG) und die Ergänzung des Anhang 1 der FFH-Richtlinie um weitere Moorgebietstypen. ${ }^{41}$ Auch zur standortangepassten Bewirtschaftung von Mooren bestehen zahlreiche gesetzliche Vorgaben. $\mathrm{Zu}$ nennen sind die gute fachliche Praxis in der Landwirtschaft sowie beihilferechtliche Anforderungen im Rahmen von Cross Compliance und Greening.

Gleichwohl ergeben sich deutliche Umsetzungs- und Vollzugsdefizite. Vor allem der Schutz von Dauergrünland auf kohlenstoffreichen Böden ist unzureichend. Hier könnte von den Möglichkeiten der EU-Direktzahlungs-Verordnung Gebrauch gemacht werden, um Dauergrünland auf kohlenstoffreichen Böden auch außerhalb der FFH-Gebietskulisse zu umweltsensiblem Dauergrünland zu erklären und klimaschädliche Grünlandumbrüche einzuschränken ${ }^{42}$ (vgl. auch Abschn. 5.2.5.4). Die Umwandlung von Acker zu Grünland bzw. Grünlandextensivierungen auf Moorflächen (z. B. extensive Beweidungsformen) können über Agrarumwelt- und Klimamaßnahmen angereizt werden. Diese Nutzungen erlauben höhere Grundwasserstände, die für die Reduzierung von THG-Emissionen erforderlich sind.

Stärker als bisher sollten kohlenstoffreiche Böden in Flussauen in den Fokus rücken. Hier ließen sich durch Deichrückverlegungen, Renaturierungen und Flächenextensivierungen neben positiven Klimaschutzwirkungen auch Ziele des Hochwasser-, Umwelt- und Naturschutzes realisieren. Voraussetzung hierfür wäre jedoch eine deutlich bessere Integration und Abstimmung der relevanten Politikbereiche ${ }^{43}$ Hochwasserschutz, Naturschutz, Landwirtschaft und Klimaschutz (vgl. Naturkapital Deutschland TEEB DE 2015, S. 208).

\footnotetext{
${ }^{40}$ Art. 6 der FFH-Richtlinie und der zugehörige Anhang $1 \mathrm{Nr} 7$ beinhalten eine Liste zu schützender Hoch- und Niedermoore. Im Einzelnen unterliegen Hochmoore, Übergangs- und Schwingrasenmoore, Torfmoor-Schlenken, Moorwälder und kalkreiche Niedermoore dem Verschlechterungsverbot nach Art. 6 (2) FFH-Richtlinie, umgesetzt über die Regelungen des $§ 33$ (1) BNatSchG.

${ }^{41} \mathrm{Zu}$ nennen sind hier bodensaure Niedermoore, artenreiches Feuchtgrünland auf Moorstandorten, Großseggenrieder und Röhrichte (vgl. Ssymank und Scherfose 2012, S. 65 f.).

${ }^{42}$ Vgl. Artikel 45 (1) S. 2 Direktzahlungs-Verordnung EU/1307/2013. Alternativ bestünde auch die Möglichkeit, einen entsprechenden GLÖZ-Standard einzuführen, wie er in den ersten Entwürfen der EU-Verordnungen auch vorgesehen war (vgl. vertiefend Freibauer et al. 2012).

${ }^{43}$ Erste Ansätze hierzu beinhaltet das Nationale Hochwasserschutzprogramm (NHWSP, vgl. LAWA 2014), welches am 24. Oktober 2014 von der Umweltministerkonferenz verabschiedet wurde.
} 
Zentrales Hemmnis des Moorbodenschutzes ist die ökonomische Vorteilhaftigkeit der landwirtschaftlichen Nutzung degenerierter Moorflächen. Daher wird es darauf ankommen, verbesserte Rahmenbedingungen für alternative Bewirtschaftungsformen wie beispielsweise extensive Grünlandnutzung sowie Paludikulturen $\mathrm{zu}$ schaffen. Paludikulturen ermöglichen eine angepasste Fortführung der landwirtschaftlichen Nutzung auf wiedervernässten Moorflächen und tragen gleichzeitig zur Akzeptanzsteigerung von Moorrenaturierungen bei (vgl. Wichtmann und Haberl 2012, S. 6). Hier ist neben einem klaren politischen Bekenntnis zu Paludikulturen vor allem die Beihilfefähigkeit sicherzustellen und eine zielgerichtete Förderung über Agrarumwelt- und Klimamaßnahmen sowie Investitionshilfen für angepasste Landtechnik bereitzustellen. Bei Jensen (2015, S. 197 f.) finden sich zudem konkrete Vorschläge zur Ausgestaltung eines Vertragsklimaschutzes auf degenerierten Moorflächen. Paludikultur-Bestände, die sich im Zuge von Sukzessionen langfristig zu schützenswerten Feuchtbiotopen weiterentwickeln können und damit dann dem gesetzlichen Biotopschutz unterliegen, sollten prinzipiell aus dem gesetzlichen Biotopschutz herausgenommen werden, um rechtliche Unsicherheiten diesbezüglich abzubauen ${ }^{44}$. Eine Akzeptanz für diese Bewirtschaftungsform könnte damit erhöht werden (vgl. Joosten und Schröder 2014, S. 15 f.).

Für die flächenhafte Moorrenaturierung durch Wiedervernässung sind ausreichende finanzielle Mittel bereitzustellen, zum Beispiel durch die Einführung eines Moorschutzfonds und die weitere Etablierung innovativer Finanzierungskonzepte wie etwa der MoorFutures. ${ }^{45}$ Da die Wiedervernässung von Mooren eine großflächige Veränderung der Landnutzung in einem bestimmten Gebiet erfordert, ergibt sich die Notwendigkeit einer planerischen Begleitung und des Einsatzes eines gut aufeinander abgestimmten Maßnahmen- und Instrumentenbündels im regionalen und lokalen Kontext. Zum Tragen kommen planerische Instrumente, gutachterliche Untersuchungen, Instrumente der Flurbereinigung, Beratung, Flächenankauf- und Flächensicherung sowie wasserbauliche Maßnahmen.

Um eine Bündelung und Koordinierung der vielfältigen Herausforderungen einer angepassten Nutzung organischer Böden inklusive der Etablierung alternativer Bewirtschaftungsformen sowie der Moorrenaturierung $\mathrm{zu}$ gewährleisten, erscheint die Initiierung einer Bundesinitiative Moorschutz (vgl. auch SRU 2012, S. 262 ff.) zielführend, in der nationale Moorschutzziele verankert, eine verlässliche Datengrundlage geschaffen und die Maßnahmenfinanzierung über einen neu einzurichtenden

\footnotetext{
${ }^{44}$ Aus Paludikultur-Beständen können sich gesetzlich geschützte Biotope (Röhrichte/Moore) entwickeln, welche automatisch unter den gesetzlichen Biotopschutz gemäß 30 (2) Nr. 2 BNatSchG fallen und zu deutlichen Bewirtschaftungseinschränkungen führen können.

${ }^{45}$ MoorFutures sind auf dem freiwilligen Zertifikatemarkt erhältliche Emissionszertifikate, mit denen unternehmerisches Kapital für Moorrenaturierungen akquiriert wird (zurzeit etabliert in Mecklenburg-Vorpommern, Brandenburg und Schleswig-Holstein, siehe auch www.moorfutures. de).
} 
Moorschutzfonds gesichert werden könnte. Auch könnte auf diese Weise eine verbesserte länderübergreifende Vernetzung der handelnden Akteure herbeigeführt und die Implementierung neuer Finanzierungsmodelle vorangetrieben werden. Eine solche Bundesinitiative könnte zudem die in mehreren Bundesländern etablierten Moorschutzprogramme aufgreifen und koordinieren. Das Land Niedersachsen hat in 2015 mit der Verabschiedung der Förderrichtlinie „Klimaschutz durch Moorentwicklung“ - als Baustein des Programms „Niedersächsische Moorlandschaften“ und finanziert über den EFRE-Fonds sowie Zuschüsse des Landes - bereits eine entsprechende Initiative gestartet. Gefördert werden freiwillige Maßnahmen zum Erhalt und zur Entwicklung von Hoch- und Niedermooren, die zu einer Reduzierung von THG-Emissionen beitragen, sowie auch innovative Ansätze zur klimaschonenden Bewirtschaftung von Moorböden.

Auch in der räumlichen Planung könnten Belange des Moorschutzes künftig verstärkt Berücksichtigung finden. Dies ist zum Beispiel möglich durch die Ausweisung raumordnerischer Vorranggebiete zur Torferhaltung und Moorschutz, die Rücknahme von Vorranggebieten zum Torfabbau oder auch die verstärkte Berücksichtigung kohlenstoffreicher Böden in der Landschaftsplanung.

\subsubsection{Anpassung des Düngemanagements}

Hinsichtlich der Anpassung des Düngemanagements in der Landwirtschaft stehen die Steigerung der Effizienz des Mineraldüngereinsatzes, der N-Ausnutzung des Wirtschaftsdüngers sowie die Etablierung überregionaler Dünger-Transporte im Fokus.

\section{Erhöhung der Effizienz des Düngereinsatzes}

Steuerungsinstrumente zur Erhöhung der Effizienz des Wirtschaftsdüngereinsatzes wurden in erster Linie im Zuge der Novellierung der Düngeverordnung (DüV) geschärft und implementiert. Hier sind fachrechtliche Vorgaben $\mathrm{zu}$ vergrößerten Lagerkapazitäten, verlängerte Ausbringungssperrfristen, verschärfte Maßgaben zur unverzüglichen Einarbeitung des Wirtschaftsdüngers und anspruchsvollere Vorschriften zu technischen Mindeststandards zu emissionsarmen Ausbringungsverfahren möglich. Die einzelnen rechtlichen Modifikationen bringen z. T. erhebliche betriebliche Anpassungsbedarfe sowie im Falle emissionsarmer Ausbringungstechniken hohe Investitionskosten und finanzielle Belastungen der landwirtschaftlichen Unternehmen mit sich, die in der Regel nur im überbetrieblichen Einsatz dieser Techniken zu realisieren sind. Begleitend sind in jedem Falle entsprechende Beratungsangebote und Fördermittel für verbesserte Güllelagerung und bodennahe Düngerausbringungstechnik bereitzustellen. In vielen Bundesländern wird dies über Agrarinvestitionsförderungsprogramme angeboten.

Weitere Impulse können von der verstärkten Umsetzung kooperativer Maßnahmen ausgehen. In der Praxis haben sich vielerorts erfolgreiche Formen der Zusammenarbeit zwischen Wasserversorgern und örtlichen Landwirten herausgebildet, die gezielt zur Reduzierung von Stickstoffüberschüssen beitragen können. So fördern etwa kommunale 
Wasserwerke gewässerschützende Bewirtschaftungsmaßnahmen der Landwirtschaft zur Reduzierung der Trinkwasseraufbereitungskosten und bewirtschaften eigene Flächen im wasserschutzoptimierten ökologischen Landbau. ${ }^{46}$ Darüber hinaus wird in einigen Bundesländern eine landwirtschaftliche Gewässerschutzberatung auf ausgewählten Grundwasserkörpern, die die Ziele der Wasserrahmenrichtlinie nicht erreichen, angeboten und durchgeführt.

\section{Etablierung überregionaler Dünger-Transporte}

Zur weiteren Etablierung überregionaler Dünger-Transporte aus Über- in Zuschussregionen bestehen verschiedene Handlungsoptionen. Zunächst bedarf es der Etablierung überregionaler Stoffkreisläufe durch interregionale Kooperationen zwischen abgebenden und aufnehmenden Regionen ${ }^{47}$ sowie der Einrichtung weiterer Nährstoffbörsen. ${ }^{48}$ Eine wichtige Voraussetzung für die Tragfähigkeit überregionaler Kooperationen stellt die Senkung der Transportkosten dar. Dies kann zum einen durch die Verbesserung der Transportwürdigkeit der Gülle selbst erfolgen (z. B. durch Separierung der nährstoffreichen Festphase), zum anderen durch den Einsatz sogenannter Kombiliner, welche sowohl Gülle (zur Verwendung in der Ackerbauregion) als auch Futtermittel (zur Verwendung in der Veredelungsregion) transportieren können und so Leerfahrten reduzieren. Für beide Maßnahmen kommen entsprechende Investitionshilfen in Frage. Darüber hinaus bedarf es eines Ausbaus der Güllelagerkapazitäten in den Ackerbauregionen, um den aufnehmenden Betrieben eine hinreichend flexible Anwendung des aufgenommenen Wirtschaftsdüngers zu ermöglichen.

\section{Verteuerung des Stickstoffdüngereinsatzes}

Der überregionale Transport organischer Düngemittel kann durch Substitutionseffekte wichtige Beiträge zur Senkung des Mineraldüngereinsatzes in Ackerbauregionen und damit zur Reduzierung von Emissionen aus der Düngerherstellung leisten. Sollen darüber hinaus weitere Impulse gesetzt werden, kommen ökonomische Instrumente wie die Einführung einer Abgabe auf mineralischen Stickstoff oder auf den betrieblichen Stickstoffüberschuss in Frage. Während die - von den Stakeholdern weitgehend abgelehnte - Stickstoffabgabe eine generelle Verteuerung des Inputfaktors (mineralischer Dünger) bewirken würde, zielt die Stickstoffüberschussabgabe auf eine Bepreisung der

\footnotetext{
${ }^{46}$ Konkrete Praxisbeispiele finden sich unter www.naturkapital-teeb.de/fallbeispiele, www.landwirtschaftskammer.de, www.nlwkn.niedersachsen.de/wasserwirtschaft.

${ }^{47}$ So etwa praktiziert zwischen der Bioenergieregion Südoldenburg und der Partnerregion Braunschweig, siehe www.bioenergie-suedoldenburg.de.

${ }^{48}$ Nährstoffbörsen (Güllebörsen) dienen in der Praxis der Vermittlung von Angebot und Nachfrage zwischen Überschuss- und Aufnahmeregionen. Grundlage sind meist Erlasse der jeweiligen Landesregierung, die die zentrale Datenverwaltung und die Kontrollmöglichkeiten durch die Landwirtschaftsbehörden regeln.
} 
Stickstoffüberschüsse landwirtschaftlicher Betriebe ab. Letztere wird in fachlichen Diskursen aufgrund der hohen Zielgenauigkeit und Lenkungswirkung als das effektivere Instrument zur Reduzierung der Stickstoffüberschüsse auf landwirtschaftlichen Flächen angesehen (so etwa SRU 2015, S. 346). Die Einführung einer solchen Abgabe wäre zwar mit einem hohen Verwaltungsaufwand und Kontrollkosten verbunden (vgl. Möckel 2014, S. 32), könnte das bestehende Ordnungsrecht jedoch durch ökonomische Anreize zum verminderten Düngemitteleinsatz sinnvoll ergänzen. In jedem Falle wäre bei der Einführung ökonomischer Anreizinstrumente sicherzustellen, dass die aufgrund der Abgabe anfallenden Einnahmen nach Deckung der administrativen Kosten in den landwirtschaftlichen Sektor zurückfließen.

\subsubsection{Substitution fossiler Energie durch Biomasse}

Eine natur- und raumverträgliche Substitution fossiler Energien durch Biomasse kann auf verschiedenen Wegen erreicht werden. Zunächst ist zur (regionalen) Verminderung des Maisanbaus - zusätzlich zur erfolgten Rücknahme überzogener Förderanreize des Erneuerbare-Energien-Gesetzes - eine anspruchsvollere Ausgestaltung sowie konsequentere Umsetzung umwelt- und agrarpolitischer Steuerungsinstrumente notwendig. Dies kann beispielsweise durch verschärfte Vorgaben zur Anbaudiversifizierung im Rahmen des Greening sowie eine intensivere Förderung der Anbaudiversifizierung über Agrarumwelt- und Klimamaßnahmen erreicht werden. Hierzu bedarf es vor allem der stärkeren Etablierung alternativer Energiepflanzen (annuell und mehrjährig) und einer weiteren Priorisierung des Einsatzes von Substraten auf Basis von Abfall- und Reststoffen sowie landwirtschaftlicher Nebenprodukte. Weitere Effekte können von einer anspruchsvolleren Ausgestaltung der Düngeverordnung ausgehen (vgl. Abschn. 5.2.5.2).

\section{Anbau alternativer einjähriger und mehrjähriger Energiepflanzen}

Um einen verstärkten Anbau alternativer einjähriger Kulturen zur energetischen Verwendung zu erreichen, bedarf es neben Forschungsbemühungen zur Erhöhung der Wirtschaftlichkeit entsprechender Kulturen und deren Gasausbeute in Biogasanlagen der Fortführung und des Ausbaus bestehender Informations- und Beratungsangebote. Für einen verstärkten Einsatz alternativer mehrjähriger Kulturen (z. B. Durchwachsene Silphie oder Miscanthus) kommt es darauf an, weitere Forschungen zur Saatgutzüchtung (Durchwachsene Silphie), Vermehrung, Anbau und Ernte sowie stofflicher und energetischer Nutzung voranzubringen, eine gezielte einzelbetriebliche Beratung zu gewährleisten und entsprechende Pilotprojekte zu fördern. So kann zur Etablierung regionaler Absatzmärkte beigetragen werden. Allerdings ist Mais die aus einzelbetrieblicher Sicht die vorzüglichste Pflanze für für Biogasanlagen. 


\section{Etablierung von Kurzumtriebsplantagen und Agroforstsystemen}

Die ebenfalls diskutierte Etablierung von Kurzumtriebsplantagen (KUP) und Agroforstsystemen (AFS) könnte befördert werden durch

- die Einführung eigenständiger GAK-Fördergrundsätze und deren Übernahme in die Länderprogramme, um die Hemmnisse der hohen Anfangsinvestitionen zu überwinden,

- die Anerkennung von KUP und AFS als produktionsintegrierte Kompensationsmaßnahmen auf Ackerflächen (unter bestimmten naturschutzfachlichen Voraussetzungen),

- die Verbesserung der wasserrechtlichen Rahmenbedingungen für KUP und AFS an Gewässerrandstreifen und in Überschwemmungsgebieten sowie

- die gezielte landwirtschaftliche Beratung, einen verbesserten Wissenstransfer und die Deckung des KUP- und AFS-Forschungsbedarfs.

Das Wasserrecht erschwert den Anbau von KUP auf Gewässerrandstreifens und in Überschwemmungsgebieten, obwohl diese zahlreiche positive Umwelteffekte bedingen (z. B. Verringerung von Stoffeintragungen intensiv genutzter landwirtschaftlicher Flächen in Gewässer, Verzögerung und Glättung von Abflussspitzen). Die $\S \S 38$ und 78 WHG könnten dahingehend angepasst werden, dass der Anbau (bzw. die Ernte) von KUP auf Ackerflächen an Fließgewässern unter bestimmten Voraussetzungen ermöglicht wird. Da ein wirtschaftlicher Anbau von KUPStreifen entlang von Fließgewässern aufgrund der notwendigen naturschutzfachlichen Anbaubeschränkungen nicht möglich ist, wären zusätzliche Anreize in Form einmaliger investiver Förderungen nötig.

Dabei ist vor allem für KUP eine räumliche Steuerung über die Landschaftsplanung notwendig, um positive Umweltwirkungen sicherzustellen und großflächige KUPMonokulturen zu vermeiden (vgl. BfN 2012).

\section{Verstärkte Biomassenutzung landwirtschaftlicher Nebenprodukte}

Die energetische Nutzung landwirtschaftlicher Nebenprodukte, z. B. Wirtschaftsdünger, Stroh, extensivem Grünlandschnitt oder Landschaftspflegematerial und Blühstreifenaufwuchs, könnte angelehnt an die in 2014 aufgehobene Einsatzstoffvergütungsklasse II des EEG 2012 gefördert werden (vgl. KLU 2014, S. 16). Darüber hinaus kommen der landwirtschaftlichen Beratung sowie weiteren Forschungsbemühungen zur stofflichen und energetischen Nutzung und Verwertung landwirtschaftlicher Nebenprodukte Bedeutung zu. Da die Böden bei vollständiger Ernte (also der kompletten Entfernung der Reststoffe) Humus und Nährstoffe verlieren würden und zum Ertragserhalt gedüngt werden müssten, sind Förderanreize auf eine nachhaltige Nutzung landwirtschaftlicher Reststoffe auszurichten. 


\subsubsection{Erhaltung und Schaffung von Treibhausgassenken}

Bei der Erhaltung und Schaffung von THG-Senken in der Landwirtschaft rückt in Deutschland neben der Moorrenaturierung der Schutz des Grünlandes in den Fokus. Für einen effektiveren Grünlanderhalt ist zunächst auf die grundsätzliche Tatsache hinzuweisen, dass Grünlandschutz zuallererst eine wirtschaftlich rentable Grünlandbewirtschaftung - z. B. durch Milchviehbetriebe und Rinder haltende Betriebe sowie Schafhalter - notwendig macht, wofür entsprechende Fördermittel bereitzustellen sind. Neben fachrechtlichen Vorgaben zur Erhaltung des Grünlands kommt seit 2015 insbesondere der Greening-Komponente zum Grünlandschutz eine zentrale Bedeutung zu. Hier bedarf es im Zuge der Halbzeitevaluierung der GAP-Reform einer Überprüfung der Gebietskulisse des umweltsensiblen Dauergrünlands. Dabei ist vor allem zu überlegen, wie die Einbeziehung kohlenstoffreicher Böden gewährleistet und wie der Abbau von Anreizen zum Grünlandumbruch durch andere Greening-Vorgaben unterbunden werden kann. ${ }^{49}$ Hinzuweisen ist auf die Tatsache, dass über das Greening zum einen nur der Landnutzer, nicht jedoch der Landeigentümer adressiert werden kann und dass zum anderen die Gefahr besteht, dass bewusst die Kürzung oder der Verlust der GreeningPrämie hingenommen wird, um eine alternative Grünlandnutzung umzusetzen.

Neben instrumentellen Modifikationen in der GAP sollten daher auch ordnungs- und planungsrechtliche Möglichkeiten für einen effektiveren Grünlanderhalt berücksichtigt werden. Hierzu sind verschiedene Instrumente im Vollzug zu stärken, maßgeblich die gute fachliche Praxis im BNatSchG (besserer Schutz kohlenstoffreicher Böden, vermehrte Festschreibung von Umbruchverboten in Schutzgebietsverordnungen), die Eingriffsregelung (Umbrüche auch auf Niedermoorstandorten als Eingriff werten) sowie das Wasserrecht (vermehrte Festschreibung von Grünlandumbruchverboten in Wasserschutzgebieten).

Effektiv umsetzen ließe sich ein vermehrter Grünlandschutz über weitere Grünlanderhaltungsgesetze auf Länderebene. Diese können innerhalb regional differenzierter Gebietskulissen Umwandlungs- und Umbruchverbote (v. a. auf organischen Böden) aussprechen. Gleichzeitig bergen sie im Gegensatz zum Grünlandschutz über das GAP-Greening nicht die Gefahr, dass durch den Verzicht auf die Greening-Prämie eine Umgehung der Vorgaben zum Grünlandschutz erfolgt und weitere Grünlandverluste hingenommen werden müssen.

Um eine vermehrte Umwandlung von Ackerland in Grünland zu erreichen, könnte die entsprechende GAK-Förderung ausgeweitet, und verstärkt in die Länderprogramme aufgenommen werden. Die Vertragslaufzeiten der Agrarumwelt- und Klimamaßnahmen

\footnotetext{
${ }^{49}$ Diese können sich durch die Bereitstellung ökologischer Vorrangflächen auf zu Ackerland umgewandelten Grünlandflächen innerhalb des tolerierten 5-\%igen Grünlandrückgangs sowie durch die Anforderungen an die Anbaudiversifizierung im Ackerland ergeben (vgl. Röder et al. 2014, S. 45).
} 
sollten möglichst verlängert werden. Die Landschaftsplanung könnte für eine räumliche Lenkung der Maßnahmen auf kohlenstoffreichen Böden herangezogen werden.

\subsubsection{Institutionelle Gestaltungsoptionen im Sektor Forstwirtschaft}

Die folgenden Ausführungen $\mathrm{zu}$ institutionellen Gestaltungsoptionen im Sektor Forstwirtschaft greifen nicht die einzelnen modellierten betrieblichen Bewirtschaftungsmaßnahmen auf. Vielmehr sollen die sich aus den Maßnahmenbündeln ergebenden Handlungsfelder, wie sie in Abschn. 3.3 vorgestellt wurden, aufgegriffen werden.

\subsubsection{1 Übergeordnete Gestaltungsoptionen im Sektor Forstwirtschaft}

Es ist festzustellen, dass in Deutschland keine aktive, unmittelbar auf Klimaschutz ausgerichtete Politik zur Bewirtschaftung der Wälder existiert (so auch Naturkapital Deutschland - TEEB DE 2015, S. 156). Die verschiedenen den Sektor betreffenden Strategien sind meist wenig konkret und nur unzureichend untereinander abgestimmt. Das einzige konkrete Politikinstrument mit entsprechenden Ansätzen ist der Waldklimafonds ${ }^{50}$, der jedoch aufgrund der geringen finanziellen Ausstattung nur eingeschränkte Effekte erwarten lässt.

Insgesamt ist zu konstatieren, dass die Klimaschutzleistung der Wälder als öffentliches Gut nicht hinreichend honoriert wird, sodass für forstliche Betriebe derzeit nur sehr geringe Anreize bestehen, nicht marktgängige Ökosystemleistungen bereitzustellen. Aktuell ist für den Landwirtschaftssektor eine um den Faktor 31,6 höhere Förderquote als in der Forstwirtschaft gegeben ${ }^{51}$, was keinesfalls die Bedeutung der Gemeinwohlleistungen des Waldes widerspiegelt (vgl. Möhring und Mestemacher 2009, S. 71). Insofern erscheint zur Honorierung der gesellschaftlichen Leistungen des Waldes eine Anpassung der bestehenden Förderinstrumente notwendig, um Maßnahmen zum Klimaschutz und zur Klimaanpassung im Privat- und Körperschaftswald stärker als

\footnotetext{
${ }^{50}$ Im Juni 2013 wurde vom Deutschen Bundestag der Waldklimafonds als Programmbestandteil des Sondervermögens Energie- und Klimafonds (EKF) verabschiedet. Die Förderrichtlinie soll zum Erhalt und zum Ausbau des $\mathrm{CO}_{2}$-Minderungspotenzials von Wald und $\mathrm{Holz}$ sowie zur Anpassung der Wälder an den Klimawandel beitragen und ist zunächst mit 7 Mio. $€$ jährlich ausgestattet.

${ }^{51}$ In 2012 etwa betrug die durchschnittliche staatliche Förderung im Privatwald gemäß den Buchführungsergebnissen der Forstwirtschaft $13 €$ /ha Holzbodenfläche (vgl. BMEL 2013, S. 5). Für den Sektor Landwirtschaft hingegen ergibt eine Addition der EU-Direktzahlungen, Zins- und Investitionszuschüsse, Agrardieselvergütungen, Ausgleichszulagen, Zahlungen aus AUKM und sonstigen Zahlungen eine durchschnittliche Fördersumme von $411 € /$ ha für das Wirtschaftsjahr 2013/2014 (vgl. BMEL 2014, S. 25).
} 
bisher anzureizen. In diesem Zusammenhang ist auch ein deutlicher Ausbau freiwilliger Vertragsnaturschutzprogramme im Wald anzusprechen. Diese können für vielfältige Maßnahmen flexibel eingesetzt werden, stehen jedoch bisher hinsichtlich der bereitgestellten Förderprogramme und -mittel deutlich hinter dem Vertragsnaturschutz in der Landwirtschaft zurück.

Zudem sind in der klimapolitischen Diskussion bei der Ausgestaltung von Förderinstrumenten und ordnungspolitischen Maßnahmen die Systemgrenzen des Waldes zu erweitern. Politische Entscheidungen, die lediglich die Senkenleistung des Waldes als Entscheidungsgrundlage heranziehen, lassen die Tatsache außer Acht, dass die Forstwirtschaft über den Holzproduktespeicher und Substitutionseffekte weitere Beiträge zum Klimaschutz leistet. In diesem Zusammenhang ist auf die Notwendigkeit hinzuweisen, verstärkt auf die Kaskadennutzung von Holzprodukten ${ }^{52}$ hinzuwirken. Die durch die Energiewende ausgelöste hohe Nachfrage nach Energieholz bedingt hingegen eine zunehmende energetische Holznutzung. Daher sollte mittels Fördermaßnahmen eine verstärkte stoffliche Nutzung von Holz angestrebt bzw. auf die Förderung der Ausweitung der energetischen Holznutzung zukünftig verzichtet werden, da hier bereits etablierte Märkte vorhanden sind.

\subsubsection{Gestaltungsoptionen zur Erhöhung der Klimaschutzleistung der Wälder}

\section{Walderhaltung}

Aus Klimaschutzsicht kommt zunächst der Walderhaltung eine hohe Bedeutung zu, da global gesehen die Entwaldung eine der größten THG-Quellen darstellt. In Deutschland ist die Walderhaltung durch den Schutz der Wälder nach $\S 9$ Bundeswaldgesetz (BWaldG) gewährleistet. Demnach sind Waldumwandlungen zwar grundsätzlich möglich, jedoch müssen diese durch Ersatzaufforstungen kompensiert werden. ${ }^{53}$

\section{Erhöhung der Vorräte und Holzentnahmen durch Aufforstung}

Zusätzliche Kohlenstoffsenken lassen sich über die Neuanlage von Wald generieren. Soll eine Steigerung der Waldfläche durch Erstaufforstung erreicht werden, sind entsprechende quantitative landespolitische Waldmehrungsziele als handlungsleitende Zielsetzung und insbesondere eine planerische Begleitung und Koordinierung der

\footnotetext{
${ }^{52}$ Kaskadennutzung bedeutet, dass Holzprodukte im Idealfall zunächst stofflich und dann energetisch verwertet werden, da sich so eine wesentlich bessere $\mathrm{CO}_{2}$-Bilanz als bei der alleinigen Holzverbrennung erzielen lässt (vgl. Knauf und Frühwald 2013, S. 28).

${ }^{53}$ Aufgrund der langen Wuchszeiträume neu begründeter Wälder kann sich dabei trotz neutraler Flächenbilanz eine Reduktion der Kohlenstoffspeicherung im Wald einstellen, wenn z. B. bei einem Straßenneubau mit entsprechender Ersatzaufforstung vorratsreiche Wälder durch vorratsarme Jungbestände ersetzt werden.
} 
Waldmehrung notwendig. In Landesentwicklungs- und Regionalplänen können Vorranggebiete für Waldmehrung mit raumordnerischem Zielcharakter festgelegt werden, gegebenenfalls unterstützt durch eine gesonderte Waldmehrungsplanung als Teil der forstlichen Rahmenplanung. Die Waldmehrung sieht sich jedoch verschiedenen Hemmnissen gegenüber, wobei vor allem das eingeschränkte Flächenpotenzial die Möglichkeiten einer solchen Klimaschutzmaßnahme limitiert. Zudem stehen rechtliche und anreiztechnische Rahmenbedingungen einer großflächigen Waldmehrung entgegen, beispielsweise die gegenüber der Landwirtschaft geringeren Flächenerträge sowie die permanente Flächenfestlegung aufgrund der rechtlichen Vorgaben zur Walderhaltung, sodass insbesondere auf landwirtschaftlichen Flächen Erstaufforstungen für den Flächeneigentümer oftmals nicht attraktiv erscheinen. Notwendig wäre daher die Wiedereinführung und verbesserte Mittelausstattung der Aufforstungsförderung in den Bundesländern, die neben der eigentlichen Erstaufforstungsförderung um die Zahlung einer Einkommensverlustprämie zu ergänzen wäre. Darüber hinaus könnten alternative Finanzierungssysteme - wie beispielsweise die Waldaktien in Mecklenburg-Vorpommern ${ }^{54}$ - weiter ausgebaut werden.

\section{Wiedervernässung von Moorwäldern}

Die Wiedervernässung von Mooren auf landwirtschaftlich genutzten Flächen wurde in Abschn. 5.2.5.1 bereits angesprochen. Gleichfalls von Bedeutung ist die Wiedervernässung von Moorwäldern, die ebenfalls sowohl Zielen des Naturschutzes als auch des Klimaschutzes dient. Zudem weist die Maßnahme aufgrund der auf Waldmoorstandorten erzielbaren Bonitäten und Holzqualitäten vergleichsweise geringe Opportunitätskosten für die Forstbetriebe auf (vgl. Abschn. 3.3.2) und stellt demnach eine kostengünstige Klimaschutzmaßnahme dar.

Intakte Waldmoore verfügen aufgrund der Bestimmungen der FFH-Richtlinie und des Bundesnaturschutzgesetzes über einen hohen Schutzstatus. Weitere Anstrengungen sind jedoch zur Wiedervernässung degradierter Moorwälder notwendig. Entsprechende Renaturierungsmaßnahmen bedürfen in erster Linie entsprechender Finanzierungsinstrumente. Neben Fördermitteln aus dem Waldklimafonds stehen hierzu insbesondere Landesmittel zur Verfügung, beispielsweise über Fördermaßnahmen zur Lebensraumverbesserung im Wald oder Waldumbaumaßnahmen. Denkbar sind auch gesonderte Förderprogramme wie etwa das Waldmoorschutzprogramm Brandenburg. Darüber hinaus besteht die Möglichkeit, eine Finanzierung über Ausgleichs- und Ersatzmaßnahmen im Rahmen der Eingriffsregelung zu realisieren.

\footnotetext{
${ }^{54}$ Sowohl Private als auch Unternehmen im Rahmen ihrer unternehmerischen Gesellschaftsverantwortung (Corporate Social Responsibility) können symbolische Waldaktien erwerben. So konnten seit 2007 auf einer Fläche von rund 70 ha mittlerweile 13 Klimawälder sukzessive aufgeforstet werden (siehe auch www.waldaktie.de).
} 
Erhöhung der Holzentnahme aus dem Wald versus Erhöhung der Vorräte im Wald Klimaschutzeffekte des Waldes könnten sich durch die Änderung der Bewirtschaftungsform bestehender Wälder ergeben. Dabei zeigt sich jedoch beim aktuellen Altersklassenaufbau der Wälder, dass weder eine Intensivierung (z. B. verkürzte Umtriebszeiten) noch eine Extensivierung (Akkumulation von Kohlenstoff in den Wäldern) vorzüglich ist, da sich die Wirkungen auf Wald- und Holzproduktespeicher wechselseitig beeinflussen und die aktuell vorherrschende Waldbewirtschaftung in Bezug auf die Kohlenstoffspeicherung die beste Bilanz aufweist (vgl. Rüter et al. 2011). Sollen dennoch waldbauliche Handlungsempfehlungen hinsichtlich Durchforstungshäufigkeit, Durchforstungsstärke, Zielstärke, Endnutzungsmenge etc. zur Umsetzung kommen, wäre dies über die Waldbaurichtlinien der Länder (mit Vorgaben für den Landesforst), Anpassungen der Zertifizierungssysteme oder Maßnahmen des Vertragsnaturschutzes möglich.

\subsubsection{Anpassung der Wälder an den Klimawandel und Vermeidung von Kohlenstoffverlusten}

Wälder können Beiträge zum Klimaschutz leisten und der Anpassung an die Klimaänderungen dienen (Erosionsschutz, Wasserrückhaltung etc.). Gleichzeitig sind sie selbst von den Klimawandelfolgen betroffen (Trockenheit, Extremwetterereignisse etc.). Dabei bestehen Wechselwirkungen und Abhängigkeiten zwischen Klimaschutz und Klimaanpassung, da stabile Wälder eine zentrale Voraussetzung für die Erhaltung der durch sie bereitgestellten Kohlenstoff-Sequestrierung darstellen.

Anpassungsmaßnahmen im Wald sind sehr vielfältig und umfassen die Baumartenwahl, Umtriebszeiten oder die Struktur der Wälder. Dabei werden recht gegensätzliche Ansatzpunkte der Klimaanpassung diskutiert, sodass keine allgemeingültigen Handlungsempfehlungen ausgesprochen werden können. Vereinfachend lassen sich zwei gegensätzliche Anpassungsstrategien unterscheiden (vgl. Winkel 2015): Die erste Strategie fokussiert auf die Intensivierung der Bewirtschaftung, indem Bestandesrisiken durch eine Senkung der Produktionsalter minimiert werden und verstärkt auf nicht heimische Baumarten gesetzt wird. Die zweite Strategie zielt auf die Etablierung naturnaher, vielfältiger und strukturreicher Wälder ab, um so die Resilienz der Wälder zu verstärken.

Eine Einflussnahme auf die beiden Strategien erfolgt über Waldumbauprogramme, die forstliche Förderung und Beratung sowie die landesrechtlichen Waldbaurichtlinien für den öffentlichen Wald. Da der zweiten Strategie positivere Wirkungen für den Biodiversitätsschutz sowie kulturelle Leistungen des Waldes zugesprochen werden (vgl. ebd.), ist insbesondere die forstliche Förderung stark hierauf ausgerichtet. Hier wird der ökologische Waldumbau, also der Umbau von naturfernen Nadelbaumreinbeständen zu naturnäheren Laub- und Mischwäldern, gefördert und zunehmend mit Notwendigkeiten der Klimaanpassung begründet. Soll dieser Waldumbau weiter befördert werden, muss vor allem eine stärkere Einbeziehung der nichtstaatlichen Waldflächen durch die Bereitstellung ausreichender Finanzmittel zur Beförderung des Waldumbaus im Privat- und 
Körperschaftswald gelingen. Ergänzend sind informelle Informations- und Beratungsangebote weiter auszubauen, um im Privat- und Körperschaftswald die Notwendigkeit und die langfristigen Vorteile des Waldumbaus zu verdeutlichen und entsprechende waldbauliche Handlungsempfehlungen vermitteln zu können.

Da im Waldumbau die Laubholzanteile tendenziell ansteigen, bedarf es einer verstärkten Substitution von Nadel- durch Laubholz im stofflichen Bereich, um den aktuellen Marktpräferenzen auch bei veränderten Laubholzanteilen gerecht werden zu können. Gleichzeitig gilt es, klimaangepasste Nadelholzarten zu kultivieren. Darüber hinaus ist eine Lösung des Wald-Wild-Konflikts ${ }^{55}$ anzustreben. Hierzu wäre insbesondere eine Reduktion der Schalenwildbestände notwendig, die durch Wildverbiss einen Waldumbau hin zu Mischbeständen deutlich hemmen.

\subsubsection{Fazit}

Zur Umsetzung eines nachhaltige(re)n Landnutzungsmanagements besteht eine Vielzahl an Instrumenten innerhalb unterschiedlicher Politikbereiche und auf allen administrativen Ebenen. Belange des Klimaschutzes und der Klimaanpassung haben in den vergangenen Jahren bereits eine weitgehende Verankerung im europäischen und deutschen Umwelt- und Planungsrecht sowie in verschiedenen Förderpolitiken erfahren. Wenngleich rechtliche Anpassungsbedarfe oder die Notwendigkeit neuer Instrumente nur vereinzelt bestehen, zeichnet sich ein Weiterentwicklungsbedarf der Regelungs- und Steuerungssysteme ab. Dieser zielt in erster Linie auf den Abbau von Umsetzungsdefiziten und Fehlanreizen ab sowie auf die stärkere Implementierung klimawandelrelevanter Belange in verschiedenen Förderpolitiken, etwa der Gemeinsamen Agrarpolitik, der Städtebauförderung sowie der Integrierten Ländlichen Entwicklung. Insbesondere ist eine stärkere Berücksichtigung der nicht marktfähigen Ökosystemleistung Klimaschutz anzustreben, was für die Etablierung eines Fonds zur Finanzierung von Klimaschutzprojekten spricht (etwa in Anlehnung an die Funktionsweise des Waldklimafonds, vgl. auch Naturkapital Deutschland TEEB DE 2015, S. 207).

Entscheidend ist das Zusammenwirken unterschiedlicher Instrumente: So sollte der Fokus darauf liegen, Zielkonflikte aufzudecken und $\mathrm{zu}$ berücksichtigen sowie instrumentelle Synergien zu nutzen, etwa zwischen Klimaschutz und Naturschutz (vgl. Schuler et al. 2014). Bei der Ausgestaltung planerischer, ordnungsrechtlicher und anreiz-

\footnotetext{
${ }^{55}$ In weiten Teilen Deutschlands sind deutlich überhöhte Schalenwildbestände zu verzeichnen. Der dadurch bedingte hohe Schalenwildverbiss führt zu Wachstumseinbußen, von dem in erster Linie die vitalen und wuchskräftigen Individuen der Baumverjüngung sowie Keimlinge betroffen sind. Aufgrund des selektiven Verbisses kommt es zudem zur Entmischung künftiger Bestände. Diese Wirkzusammenhänge erschweren einen Waldumbau hin zu Mischbeständen deutlich (vgl. Ammer et al. 2010).
} 
technischer Instrumente ist eine instrumentelle Überfrachtung möglichst $\mathrm{zu}$ vermeiden. Insbesondere ordnungsrechtliche Vorgaben sollten maßvoll eingesetzt werden und nur dann zum Einsatz kommen, wenn kooperative, anreiztechnische und informative Ansätze nicht ausreichen. Vor dem Hintergrund knapper öffentlicher Gelder kann es zielführend sein, vor allem solche Maßnahmen prioritär umzusetzen, die auch losgelöst von Klimaschutz und Klimaanpassung positive Umweltwirkungen entfalten (no-regretMaßnahmen).

Für ein nachhaltiges Landnutzungsmanagement ist vor dem Hintergrund der komplexen Wechselwirkungen und Zielkonflikte zwischen und innerhalb der Landnutzungssektoren darauf hinzuwirken, verstärkt integrierte und koordinierende Planungs- und Handlungsansätze zur Anwendung zu bringen. Besondere Bedeutung kommt hier der räumlichen Gesamtplanung zu. Diese kann im Rahmen der Raumordnung sowie der Kommunalplanung dazu beitragen, die im Zuge des Klimawandels und der Energiewende zunehmenden Flächennutzungskonflikte frühzeitig zu erkennen und koordinierend auf diese einzuwirken. Zudem können auf Ebene der Landes- und Regionalplanung Klimaschutzmaßnahmen sowie Maßnahmen zur Strategie „Klimaanpassung“" vorbereitet und entsprechende Flächen gesichert werden.

\subsection{Landnutzung und Klimawandel im transdisziplinären Diskurs: Wissenschaft und Stakeholder in Interaktion}

Annett Steinführer, Meike Hellmich, Rosemarie Siebert und Reimund Steinhäußer

\section{Zusammenfassung}

CC-LandStraD war nicht nur als inter-, sondern auch als transdisziplinäres Projekt konzipiert worden. Das Erfahrungs- und Praxiswissen relevanter, mit Landnutzung mindestens semiprofessionell befasster gesellschaftlicher Akteure wurde für den Forschungsprozess, die Validierung von Zwischenergebnissen und die Praxisrelevanz der zu erzielenden Ergebnisse für so relevant gehalten, dass bundesweite und regional tätige Stakeholder kontinuierlich zur Beteiligung am Projekt eingeladen wurden. Dies erfolgte durch Workshops, leitfadengestützte Interviews und Fokusgruppendiskussionen. Mittels Feedbackbögen und -interviews wurden Erwartungen und erzielte Ergebnisse vergleichend reflektiert. Inhaltlich leisteten die Stakeholder Beiträge insbesondere zu den landnutzungsbezogenen Klimaschutz- und Klimaanpassungsmaßnahmen sowie deren Ausgestaltung in den Strategien (Klimaschutz, Bioenergie, Natur- und Umweltschutz, Klimaanpassung). Der Artikel stellt den Prozess und seine Ergebnisse dar und reflektiert die Beteiligung und inhaltlichen Anregungen der Stakeholder sowie methodische Fragen, wie etwa den Übersetzungsaufwand zwischen Modellierungswissenschaften und Anwendungserwartungen. 


\subsubsection{Stakeholder-Beteiligung in der Umweltforschung}

Die Beteiligung unterschiedlicher Akteure von außerhalb der Wissenschaft ist in der sozialökologischen Forschung in den vergangenen Jahren nahezu zur Routine geworden. Die erkenntnistheoretischen und gesellschaftspolitischen Hintergründe dieses Paradigmenwechsels reichen in den Risikodiskurs der 1980er-Jahre zurück. Dieser hatte vor allem die Rückkopplungseffekte hochtechnisierter Systeme sowie ihre von der Wissenschaft nicht intendierten oder ihr unbekannten Folgeprobleme thematisiert. Letztere spielten eine zentrale Rolle für die Erosion des Glaubens an den technischen Fortschritt und die Beherrschbarkeit der (Um-)Welt auf Grundlage naturwissenschaftlichen Erkenntnisgewinns. So entstand eine neue anwendungsorientierte, inter- und transdisziplinäre Umweltforschung, die zur Lösung praktischer Probleme beitragen und dafür naturwissenschaftliche Expertise, sozialwissenschaftliche Methoden und Konzepte sowie das Erfahrungswissen der Praxis möglichst gleichberechtigt einbinden möchte. In Deutschland verbindet sich dieser Zugang bis heute in besonderer Weise mit dem sozialökologischen Paradigma, das explizit nach ,neuen Wissensformen“ sucht, „die auf lebenspraktische gesellschaftliche Probleme bezogen sind und zu deren Bewältigung beitragen“ (Becker und Jahn 2006, S. 15). Eine neuere Entwicklung ist die Citizen Science, eine Forschung unter Bürgerbeteiligung, beispielsweise im Bereich gewünschter oder unerwünschter Biodiversität, wenn etwa die Bewohner einer Stadt oder Region gebeten werden, Wildtiere in urbanen Räumen zu zählen oder die Flora von Bergwiesen zu bestimmen (z. B. Finke 2014).

Parallel erfolgte neben der prinzipiellen Hinterfragung und kritischen Reflexion tradierter Wissensquellen und -formen eine Veränderung umweltpolitischer Governancestrukturen: Die ,aktive Beteiligung aller interessierten Stellen“, wie die Formulierung beispielsweise in Artikel 14 der im Jahr 2000 verabschiedeten EU-Wasserrahmenrichtlinie (WRRL) heißt, hat sich mittlerweile als Standardanforderung an die europäische Umweltpolitik durchgesetzt und schlägt sich seit langem in konkreten Instrumenten (etwa der Strategischen Umweltprüfung (SUP) oder der Umweltverträglichkeitsprüfung (UVP)) sowie verschiedenen europäischen Richtlinien (wie der Hochwasserrisikomanagement-Richtlinie (HWRM-RL) oder bereits 1982 der Richtlinie zur Beherrschung der Gefahren bei schweren Unfällen mit gefährlichen Stoffen (Seveso-RL)) nieder. Bürgerbeteiligung hat dabei im Zeitverlauf zweifellos an Bedeutung gewonnen. Inwiefern es sich aber bei den zahlreichen Beteiligungsprozessen in den unterschiedlichen Kontexten und Arenen um wirkliche Partizipation oder um Alibipolitik handelt, ist umstritten und im Einzelfall verschieden. Bereits 1969 hatte Sherry Arnstein zwischen echter und vermeintlicher Partizipation sowie Nicht-Beteiligung unterschieden. Lediglich die drei Stufen „Partnerschaft“, „Machtdelegation“ und „Bürgerkontrolle“ definierte sie in ihrer ladder of participation als wirkliche Beteiligung bzw. Mitwirkung. „Vorgänge der Konsultation“ und „Information der Bevölkerung“ zählte sie hingegen nicht dazu, da nicht 
gesichert sei, dass die Hinweise und Anregungen der Bevölkerung von der Exekutive tatsächlich beachtet würden (Arnstein 1969, bes. S. 217).

Forschungsarbeiten begleiteten die Umsetzung der zahlreichen europäischen Richtlinien und deren praktische Probleme und trugen so ebenfalls zu einer neuen sozialwissenschaftlichen Umweltforschung bei, bei der partizipative Governance, Beteiligung und Nicht-Beteiligung unterschiedlicher sozialer Gruppen sowie deren Bedingungsfaktoren und Hindernisse im Mittelpunkt stehen, etwa im Hinblick auf Wassermanagement (Jessel und Jacobs 2005), Risikoanalysen (Glicken 2000), Prozesse der Risikokommunikation (De Marchi 1991) oder das Management von Hochwasserrisiken (Wehn et al. 2015; Begg et al. 2011; Steinführer et al. 2009). Die von den Umweltproblemen oder der Instrumentenumsetzung Betroffenen werden dabei in unterschiedlichem Maße in die Forschungen mit einbezogen. Ähnlich wie in Arnsteins ladder of participation lässt sich diese Beteiligung von Informiert- über Konsultiert-Werden bis hin zum Mit-Entscheiden abstufen.

In Deutschland hat sich für die Einbindung nichtwissenschaftlicher Akteure in Forschungsprojekte das Konzept der Transdisziplinarität etabliert. Darunter wird ein über Einzeldisziplinen hinausgehender, spezifischer erkenntnistheoretischer und methodologischer Zugang der Umwelt- und Raumwissenschaften verstanden, der sich an praktischen Problemen orientiert. Außerhalb der Wissenschaft tätige Akteure sogenannte Stakeholder - werden als Praxisexperten und/oder als Vertreter bestimmter Interessen ,in einer anderen Rolle als der des Wissenschaftlers an einem Problemlösungsprozess beteiligt“" (Förster et al. 2001, S. 146; vgl. auch Zierhofer und Burger 2007; Renn 2008; Haber und Bückmann 2013, S. 32 f.). Eine Quelle des Konzepts sind Betriebswirtschaft und Strategisches Management, die eine konzeptionelle Erweiterung vom Anteilseigner (shareholder) auf breitere Interessengruppen (stakeholder) vornahmen (Freeman 1984, 2004). Dies hatte zur Folge, dass das Stakeholder-Konzept im Laufe der letzten Jahre sowohl in weitere Felder der Management- und Organisationsforschung als auch in verschiedenste Bereiche der Politik- und Entwicklungsforschung sowie, wie bereits ausgeführt, der Umweltwissenschaften Eingang gefunden hat (Reed et al. 2009). ${ }^{56}$ In den Sozialwissenschaften gilt der Stakeholder als Person oder Gruppe, deren Mitwirkung an politischen Entscheidungsprozessen aufgrund legislativer Vorgaben

\footnotetext{
${ }^{56} \mathrm{Als}$,Stakeholder“ wird in älteren Englischwörterbüchern ein unparteiischer Verwalter von Wetteinsätzen bezeichnet (vgl. z. B. Langenscheidts Enzyklopädisches Wörterbuch der englischen und deutschen Sprache; vgl. Springer 1963, S. 1366). In der Gegenwart bezieht sich seine Bedeutung im ökonomischen Kontext im Unterschied zu Anteilseignern etwa einer Aktiengesellschaft (shareholder) auf darüber hinausgehende Personenkreise (z. B. Beschäftigte oder Kunden), die ebenfalls ein legitimes Interesse am Unternehmen haben. Etymologisch ist es auf stake (Stab, der z. B. zum Abstecken von Land verwendet wurde) und hold (halten) zurückzuführen. Mit der abgeleiteten Verbform to stake verband sich ab dem frühen 16. Jahrhundert auch die Bedeutung von ,etwas riskieren, um etwas wetten“. Daraus erklärt sich auch die Bedeutung von stake als „Anteil, (Wett-) Einsatz“(Online Etymology Dictionary 2015).
} 
als notwendig erachtet wird oder deren freiwillige Beteiligung zur Vermeidung oder Verminderung von Konflikten dient. Eine Definition jenseits der Bedeutung von „Anteil haben" steht aus. Auch fehlt eine theoretische Orientierung, um abzugrenzen, wer woran zu beteiligen ist (Achterkamp und Vos 2008). Im internationalen und mittlerweile auch im deutschsprachigen Umwelt- und Nachhaltigkeitsdiskurs bezeichnet der Begriff „Stakeholder“ Personen oder Organisationen mit einem legitimen Interesse an einer Sache (Brugha und Varvasovszky 2000). Die Legitimität ergibt sich z. B. dadurch, dass diese Personen bzw. Organisationen von Entscheidungen in dieser Sache (etwa eines anderen Landnutzers) direkt oder indirekt in ihren eigenen Interessen (z. B. wirtschaftlicher Art) betroffen sind bzw. sein können.

Im hier dokumentierten Forschungsprozess des CC-LandStraD-Projektes wurden unter „Stakeholder“ einerseits alle organisierten Akteure (z. B. Behörden, Verbände oder andere Interessenvertretungen) verstanden, die ein (semi-)professionelles Interesse an Landnutzung haben und artikulieren, die Landnutzung in der einen oder anderen Form beeinflussen bzw. verändern oder von Landnutzungswandel betroffen sind (vgl. Abschn. 1.3). Dies sind zum einen im Rahmen formaler Organisationen handelnde Akteure, die nicht notwendigerweise selbst direkt von Landnutzungsveränderungen Betroffene sind, sondern als deren Vertreter bzw. in deren Auftrag agieren. Die Formulierung ,(semi-)professionell“ zielt darauf, dass die individuellen Vertreter dieser Stakeholder in der Regel in ihrer beruflichen Tätigkeit als Akteure der Landnutzung handeln oder aber, im Falle einer ehrenamtlichen Tätigkeit, einen oft beträchtlichen Teil ihrer Freizeit in diese Aktivitäten investieren. Neben privaten berufsständischen Interessenvertretungen und Zusammenschlüssen in den verschiedenen Landnutzungssektoren wurden auch nicht-private Interessenvertretungen, wie z. B. Träger öffentlicher Belange oder Umweltverbände aus den Landnutzungssektoren Landwirtschaft, Forstwirtschaft sowie Siedlung und Verkehr, eingebunden. Zum anderen wurden auf regionaler Ebene, also in den beiden Fokusregionen, auch nicht-organisierte Akteure, z. B. individuelle Landnutzer wie Land- und Forstwirte, als Stakeholder verstanden und durch das Projekt adressiert.

\subsubsection{Ziele des transdisziplinären Diskurses}

Das Verbundprojekt CC-LandStraD hatte zum Ziel, nachhaltige und gesellschaftlich akzeptierte Landnutzungsstrategien im Kontext von Klimaschutz und Klimaanpassung $\mathrm{zu}$ entwickeln (vgl. Abschn. 1.1). Neben naturwissenschaftlicher und ökonomischer Expertise sowie punktuell den Einstellungen der Bevölkerung (vgl. Abschn. 5.1) sollte hierfür auch das Erfahrungs- und Praxiswissen relevanter - also mit Landnutzung mindestens semiprofessionell befasster - gesellschaftlicher Akteure eingebunden werden. Bei deren Auswahl war der spezifische räumliche Zuschnitt des Projektes mit seinem bundesweiten Ansatz einerseits und der vertiefenden Untersuchung in den beiden 
Fokusregionen Rhein (Rhein-Sieg-Kreis und Rheinisch-Bergischer Kreis) und Altmark (Altmarkkreis Salzwedel und Landkreis Stendal) andererseits zu beachten (vgl. Abschn. 1.2).

Hauptziel der Stakeholder-Beteiligung war es, die vielfältigen Ansprüche und Erwartungen unterschiedlicher Akteure in Bezug auf aktuelle und zukünftige Landnutzungsentwicklungen $\mathrm{zu}$ berücksichtigen. Konkret waren im Projektverlauf Maßnahmen der Landbewirtschaftung (z. B. Ausweisung von Hochwasserschutzgebieten im Sektor Siedlung, bestimmte Durchforstungsstärken im Sektor Forst oder eine angepasste Grünlandnutzung auf organischen Böden im Sektor Landwirtschaft) zu beurteilen. Im zweiten Schritt sollten die Stakeholder die Maßnahmenbündel - also die im Projekt entwickelten und modellierten Landnutzungsstrategien (Klimaschutz/ KS, Bioenergie/BE, Natur- und Umweltschutz/NUS sowie Klimaanpassung/KA; vgl. Abschn. 4.1) - anhand verschiedener Kriterien bewerten. Abschließend interessierte ihr Feedback zu den Forschungsergebnissen und deren Praxisrelevanz.

Der transdisziplinäre Diskurs wurde als projektbegleitender Austausch konzipiert, der im Wesentlichen auf sektoralen Workshops sowie Fokusgruppen basierte und durch Interviews ergänzt wurde. Die beiden Prozesse - auf bundesweiter Ebene und in den zwei Fokusregionen - folgten keinem vorab im Detail festgelegten Design, sondern wurden je nach Arbeitsstand und Zwischenergebnissen der Modellierungsbausteine und dem sich daraus gegebenenfalls ergebenden Bedarf der Fachwissenschaftler situativ weiterentwickelt. Die Beteiligungsprozesse auf den beiden räumlichen Ebenen waren aufeinander abgestimmt, unterschieden sich aber in einigen Details (vgl. Abb. 5.10 sowie die weiteren Ausführungen).

Neben der prozessualen Komponente - der Begleitung des Forschungsprojektes und der damit verbundenen kontinuierlichen Übersetzungsarbeit zwischen Modellierungswissenschaften und Praxisinteressen - standen aus einer sozialwissenschaftlichen Perspektive die Bedeutung des Klimawandels in der Tätigkeit relevanter Landnutzungsakteure in Deutschland sowie Fragen inter- und intrasektoraler Landnutzungskonflikte im Mittelpunkt des Forschungsinteresses (Hellmich und Steinführer 2012; Steinhäußer et al. 2015):

- Klimawandel wird in den Sozialwissenschaften unter zwei Gesichtspunkten diskutiert: als Anpassung an die als unausweichlich erachteten Folgen (wie häufigere und/oder stärker ausgeprägte Extremereignisse, wie Hitzewellen oder Hochwasser) sowie als Anstrengung von Individuen, Unternehmen, Verbänden, Kommunen und Nationalstaaten zur Senkung von THG-Emissionen (Voss 2010). Das Besondere an CC-LandStraD in diesem Zusammenhang war, dass erstmals alle drei großen flächennutzenden Sektoren in Deutschland in ihren Bemühungen um Klimaschutz und, in den Sektoren Forstwirtschaft sowie Siedlung und Verkehr, um Klimaanpassung betrachtet wurden (vgl. auch Gömann et al. 2015). 


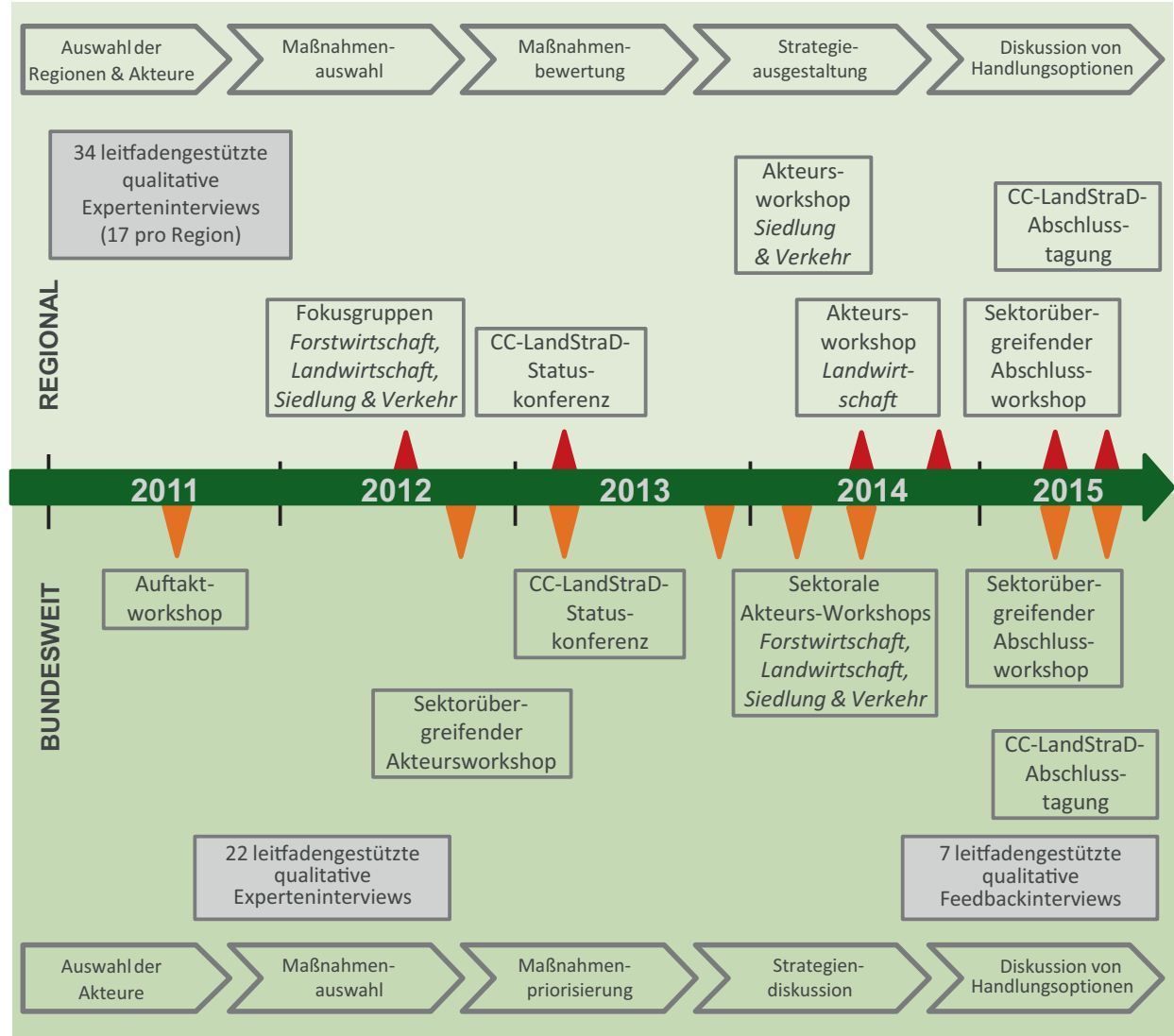

Abb. 5.10 Überblick über die Formen der Stakeholder-Beteiligung auf bundesweiter Ebene und in den Fokusregionen. (Quelle: Thünen-Institut/J. Fick, graphische Umsetzung: Nina Röhrig)

- Landnutzungskonflikte waren zunächst von Landnutzungskonkurrenzen abzugrenzen. ${ }^{57}$ Letztere entstehen dadurch, dass Flächen meist für unterschiedliche Nutzungen geeignet sind. So kann ein Acker zur Biomasseproduktion für energetische Zwecke oder alternativ zur Nahrungsmittelerzeugung genutzt werden. $\mathrm{Zu}$ Landnutzungskonflikten kommt es, wenn a) unterschiedliche Akteure einander ausschließende Nutzungsinteressen an ein und dieselbe Fläche richten oder wenn b) gesellschaftliche Interessengruppen bestimmte Landnutzungen als Problem erklären und davon ausgehend Nutzungsveränderungen bzw. -einschränkungen oder einen Nutzungsverzicht erreichen wollen (vgl. auch Arlt und Pfeil 1996; von der Dunk et al.

${ }^{57}$ Zum Teil werden beide Begriffe in der Literatur synonym oder zumindest nicht trennscharf verwendet (z. B. Rösch et al. 2008). 
2011). Landnutzungskonflikte sind stets Interaktionen zwischen individuellen oder korporativen Akteuren, die entweder nicht kosten- oder nicht flächenneutral lösbar sind. In diesem Beitrag interessieren uns sowohl intra- als auch intersektorale Landnutzungskonflikte.

\subsubsection{Wissenschaft und Praxis in Interaktion: der Prozess}

\subsubsection{Stakeholder-Auswahl}

\section{Bundesweiter Beteiligungsprozess}

Die Auswahl der bundesweiten Stakeholder fand in einem iterativen Prozess statt. Zunächst erfolgte eine Kategorisierung von Akteuren der Landnutzung nach institutionellen Zugehörigkeiten (Politik, Verwaltung, Interessenverbände und wissenschaftliche Einrichtungen). Außerdem wurden, ausgehend von den drei aus Projektsicht relevanten Hauptsektoren der Landnutzung und unter Berücksichtigung weiterer Flächenansprüche, die Interessenverbände und Ämter in sieben inhaltliche Gruppen unterschieden: Landwirtschaft, Forstwirtschaft, Siedlung und Verkehr, Natur- und Umweltschutz, Energie und Rohstoffe, Wasser sowie Sonstige (z. B. Tourismusverbände, Versicherungen, Wissenschaft u. a.).

Anhand dieser Systematisierung fand eine Internet- und Literaturrecherche statt, um bundesweit aktive Akteure der Landnutzung zu identifizieren. Diese Recherche ergab eine Liste von insgesamt 147 Organisationen. Diese Anzahl lag bei weitem über der Größenordnung, die für eine aktive Projektbeteiligung (etwa durch Workshops und Interviews) berücksichtigt werden konnte. Ein Auswahlprozess der erfassten Stakeholder nach ihrer Relevanz für das Forschungsvorhaben war notwendig. Deshalb wurde im Projektkonsortium eine Expertenbefragung durchgeführt. Zugleich konnte so sichergestellt werden, dass für alle Sektoren und Teilthemen des Projekts relevante Vertreter ausgewählt werden. Diese projektinterne Bewertung beschränkte sich auf den Bereich der Interessenverbände, da sich die Situation auf Politik- und Verwaltungsebene übersichtlicher darstellte und eine vergleichsweise geringe Anzahl relevanter Akteure identifiziert werden konnte. Für die Auswahl der Interessenverbände wurden drei Kriterien vorgegeben, von denen mindestens eines gelten musste: Die Organisation sollte 1) einen direkten oder indirekten Einfluss auf die Landnutzung in Deutschland haben, 2) in der Landnutzung selbst von Klimawandel betroffen oder Mitverursacher oder beides und schließlich 3) öffentlichkeitswirksam in Bezug auf Landnutzungsoptionen und/ oder Landnutzungskonflikte tätig sein. Um auch kleine oder weniger bekannte Verbände zu berücksichtigen, wurde eine Zusatzfrage nach drei Stakeholdern gestellt, die aufgrund besonderer Aktivitäten oder Merkmale nicht vergessen werden sollten. Mit diesem „Joker“ bestand die Möglichkeit, auf Stakeholder zu verweisen, die beispielsweise eine bestimmte, anderweitig nicht berücksichtigte, Landnutzungsform oder Sichtweise auf Landnutzungs- bzw. Klimawandel vertreten. In diesem Zusammenhang trat 
unter anderem die Frage auf, wie man mit Interessengruppen für Themen, die nur auf lokaler Ebene für bestimmte Regionen hohe Bedeutung haben, bundesweit aber eher vernachlässigt werden können, umgehen sollte. Ein typisches Beispiel hierfür ist der Abbau bestimmter Rohstoffe, wie Braunkohle, und damit verbundener Interessenlagen. Es wurde vor dem Hintergrund der Projektausrichtung und -ziele entschieden, solche Stakeholder nicht zu beteiligen.

Auf diese Weise wurden 62 Verbände und Organisationen als relevant bestimmt. Diese Expertenbewertung stellte die Grundlage für die Auswahl der im Projekt zu beteiligenden Akteure dar, wurde jedoch im späteren Projektverlauf noch geringfügig modifiziert, um ein möglichst vielfältiges und breites sowie aktuelles Spektrum der Landnutzung in Deutschland abzubilden. 70 organisierte Interessenvertreter bildeten den Ausgangspunkt für den bundesweiten Beteiligungsprozess. 51 von ihnen kamen aus den drei im Projekt vorrangig betrachteten Landnutzungssektoren Land- und Forstwirtschaft sowie Siedlung und Verkehr (mit Schwerpunkt auf der Siedlungsentwicklung). 17 Akteure des Natur- und Umweltschutzes wurden ebenfalls adressiert, ohne diesen weiterhin als „Sektor“ zu bezeichnen. Vielmehr wurde Naturschutz als ein an die drei Landnutzungssektoren gerichteter weiterer Anspruch verstanden und mit den sektoralen Perspektiven zusammengebracht (vgl. auch Tab. 5.8). Hinzu kamen zwei weitere Akteure der Kategorie „Andere“ mit einem übersektoralen Landnutzungsbezug.

\section{Regionaler Beteiligungsprozess}

Im Vorfeld der Forschungsarbeiten in den Fokusregionen Altmark und Rhein wurde auf Grundlage einer Internetrecherche der Kontakt mit Schlüsselpersonen und -organisationen gesucht. So entstand ein erster Überblick über die regionalen Akteurslandschaften. Als wichtiger Partner in der Region Altmark wurde der Regionalverein Altmark e. V. identifiziert. Er stellt mit über 60 Mitgliedern eine neue Form der regionalen Kooperation dar, in der Unternehmen mit Entscheidungsträgern aus Politik und Verwaltung sowie Verbänden und Vereinen zusammenarbeiten. $\mathrm{Zu}$ Projektbeginn wurden auf einer Sitzung des Regionalvereins das Projekt vorgestellt und erste regionale Problemstellungen aus Sicht der Akteure in die Forschungsarbeit aufgenommen. In der Fokusregion Rhein konnte kein in beiden Landkreisen agierender Partner identifiziert werden. Hier war die Unterstützung des Ministeriums für Klimaschutz, Umwelt, Landwirtschaft, Natur- und Verbraucherschutz Nordrhein-Westfalen (MKULNV) sehr hilfreich. Das Projekt und seine Ziele wurden 2011 im Ministerium vorgestellt und diskutiert.

In der Startphase wurden in beiden Fokusregionen relevante Experten aus den Bereichen Landwirtschaft, Forstwirtschaft, Naturschutz, Siedlung und Verkehr identifiziert, erste Ansprechpartner ausgewählt und kontaktiert. Diese stammten aus berufsständischen Interessenvertretungen der Landwirtschaft, unteren Landesbehörden, Landesbetrieben sowie gemeinnützigen Unternehmen mit Landnutzungsbezug sowie aus Naturschutzverbänden. Die Auswahl der Stakeholder für die Altmark wurde mit Ansprechpartnern des Regionalvereins Altmark abgestimmt und auf Relevanz für Land- 
Tab. 5.8 Beteiligungsformen und tatsächliche Beteiligung am bundesweiten Stakeholderprozess. (Quelle: Thünen-Institut/A. Steinführer)

\begin{tabular}{|c|c|c|c|c|c|c|c|c|c|c|}
\hline \multirow[t]{2}{*}{$\begin{array}{l}\text { Sektor/ } \\
\text { Bereich }\end{array}$} & \multirow[t]{2}{*}{$\begin{array}{l}\text { Identi- } \\
\text { fiziert }\end{array}$} & \multirow[t]{2}{*}{$\begin{array}{l}\text { Inter- } \\
\text { views }\end{array}$} & \multicolumn{2}{|c|}{$\begin{array}{l}\text { Workshop } 1 \\
\text { (2011) }\end{array}$} & \multicolumn{2}{|c|}{$\begin{array}{l}\text { Workshop } 2 \\
\text { (2012) }\end{array}$} & \multicolumn{2}{|c|}{\begin{tabular}{|l} 
Sektorale \\
Workshops \\
$(\mathbf{2 0 1 3} / 2014)$ \\
\end{tabular}} & \multicolumn{2}{|c|}{$\begin{array}{l}\text { Abschluss- } \\
\text { veranstaltung } \\
(\mathbf{2 0 1 5})\end{array}$} \\
\hline & & & $\begin{array}{l}\text { Ein- } \\
\text { ladung }\end{array}$ & \begin{tabular}{|l|} 
Teil- \\
nahme
\end{tabular} & \begin{tabular}{|l|} 
Ein- \\
ladung
\end{tabular} & \begin{tabular}{|l|}
$\begin{array}{l}\text { Teil- } \\
\text { nahme }\end{array}$ \\
\end{tabular} & \begin{tabular}{|l|} 
Ein- \\
ladung
\end{tabular} & $\begin{array}{l}\text { Teil- } \\
\text { nahme }\end{array}$ & \begin{tabular}{|l|} 
Ein- \\
ladung
\end{tabular} & $\begin{array}{l}\text { Teil- } \\
\text { nahme }\end{array}$ \\
\hline $\begin{array}{l}\text { Landwirt- } \\
\text { schaft }\end{array}$ & 21 & 7 & 9 & 3 & 13 & 2 & 19 & 5 & 18 & 12 \\
\hline Forst & 13 & 5 & 5 & 1 & 9 & 2 & 12 & 5 & 10 & 5 \\
\hline $\begin{array}{l}\text { Siedlung } \\
\text { und Ver- } \\
\text { kehr }\end{array}$ & 17 & 3 & 8 & 2 & 6 & 2 & 15 & 3 & 7 & 4 \\
\hline $\begin{array}{l}\text { Natur- } \\
\text { und } \\
\text { Umwelt- } \\
\text { schutz }\end{array}$ & 17 & 4 & 8 & 5 & 7 & 3 & 6 & 1 & 15 & 5 \\
\hline $\begin{array}{l}\text { Energie } \\
\text { und Roh- } \\
\text { stoffe }\end{array}$ & 25 & 1 & 2 & 1 & 0 & 0 & 1 & 0 & 2 & 1 \\
\hline Wasser & 9 & 0 & 4 & 0 & 0 & 0 & 0 & 0 & 0 & 0 \\
\hline Andere $^{\mathrm{a}}$ & 15 & 2 & 4 & 1 & 9 & 3 & 1 & 0 & 6 & 0 \\
\hline Gesamt & 117 & 22 & 40 & 13 & 44 & 12 & 54 & 14 & 58 & 27 \\
\hline
\end{tabular}

${ }^{\mathrm{a} E i n s c h l i e ß l i c h ~ W i s s e n s c h a f t ~}$

nutzungsentscheidungen in der Region geprüft. Für die Fokusregion Rhein erfolgte die Ansprache der Stakeholder in enger Kooperation mit Wissenschaftlern des Konsortiums, die über spezifische Kenntnisse der Region verfügten.

Nach dieser Startphase wurde die Stakeholder-Auswahl während der nachfolgend durchgeführten Experteninterviews mittels Schneeballverfahren fortgesetzt, indem die interviewten Experten am Ende des Gesprächs auf Nachfrage weitere Personen benannten, die für die Landnutzung der Region als relevant eingestuft wurden (Biernacki und Waldorf 1981).

\subsubsection{Beteiligungsformen und tatsächliche Beteiligung}

Der transdisziplinäre Ansatz in CC-LandStraD setzte vor allem auf interaktive Beteiligungsformen, wie sektorübergreifende und sektorale Workshops, Fokusgruppen, leitfadengestützte Interviews und Feedbackbögen. Ihre Funktionen reichten von wechselseitiger Information über Konsultation bis hin zur Beeinflussung wissenschaftlicher Entscheidungen im Projektverlauf. Darüber hinaus wurden die Stakeholder halbjährlich per Newsletter über den Stand des Projektes und die Fortschritte in den Teilprojekten 
informiert. Weitere Informationen, etwa Hinweise auf Publikationen oder populärwissenschaftliche Projektergebnisse (z. B. Filme zur Landnutzung oder eine Ausstellung zum aktuellen und historischen Landnutzungswandel), standen auf der Projektwebseite zur Verfügung. Der folgende Abschnitt konzentriert sich auf die interaktiven Beteiligungsformen - also jene mit einem stärker partizipativen Charakter.

\section{Bundesweiter Beteiligungsprozess}

Auf Bundesebene wurden sektorübergreifende und sektorale Workshops sowie leitfadengestützte Experteninterviews durchgeführt.

Der sektorübergreifende Auftaktworkshop (2011) sollte die ausgewählten Stakeholder über das Projekt informieren und zur langfristigen Teilnahme am Beteiligungsprozess einladen. Außerdem erfolgte eine Kartenabfrage zu den aus Sicht der Stakeholder bedeutendsten aktuellen Landnutzungskonflikten, den Potenzialen der Landnutzung im Bereich Klimaschutz sowie erwarteten Folgen des Klimawandels. Zur inhaltlichen Vorbereitung der Stakeholder und zur Einstimmung auf das Forschungsprojekt wurde vorab eine Tischvorlage versendet. Diese stellte die Zielsetzung des Projektes sowie wesentliche Trends der Flächeninanspruchnahme in Deutschland dar.

Im Anschluss fanden leitfadengestützte Experteninterviews statt, deren Ziel es war, die Perspektiven und Bewertungen unterschiedlicher Interessengruppen hinsichtlich aktueller sowie für die Zukunft erwarteter sektorinterner und -übergreifender Landnutzungskonflikte im Detail zu erheben. Experteninterviews sind ein erprobtes Instrument in der Umweltforschung, die es Wissenschaftlern ermöglichen, tiefe Einblicke in ihren jeweiligen Untersuchungsgegenstand zu erhalten (Reed et al. 2009; vgl. Tab. 5.8). Der Schwerpunkt des Forschungsinteresses lag auf der Frage, welche flächenbezogenen Maßnahmen die Vertreter unterschiedlicher Landnutzungssektoren für sinnvoll erachten, um einerseits einen Beitrag zum Klimaschutz zu leisten und sich andererseits an erwartete Folgen des Klimawandels anzupassen. Da sich für das Gesamtprojekt zu diesem Zeitpunkt eine fachliche Konzentration auf die Sektoren Landwirtschaft, Forstwirtschaft, Siedlungswesen sowie Natur- und Umweltschutz abzeichnete, konzentrierten sich auch die Interviews auf diese Landnutzungssektoren bzw. -ansprüche. 28 Interviewpartner aus diesen vier Bereichen (sowie zusätzlich ein Vertreter der Bioenergiebranche und zwei aus dem Wassersektor) wurden für ein Interview angefragt. Aufgrund von sechs Ablehnungen konnten 22 Interviews realisiert werden (vgl. Tab. 5.8). Befragt wurden sowohl Akteure von Nichtregierungsorganisationen, Beschäftigte von Bundesbehörden sowie eine Vertreterin einer Bund-Länder-Arbeitsgemeinschaft.

Die Interviewleitfäden basierten auf Sekundäranalysen vorhandener Forschungsliteratur, auf Selbstdarstellungen der jeweiligen Organisation im Internet und, wenn vorhanden, in Verbandszeitschriften sowie auf Diskussionen mit den jeweiligen Fachexperten des Projektkonsortiums. Für jeden Sektor wurde zunächst ein Leitfaden entwickelt, der anschließend auf den konkreten Gesprächspartner zugeschnitten wurde. 
Dabei kamen weitgehend offene Fragen ohne Antwortvorgaben zur Anwendung - auch Maßnahmen des Klimaschutzes und der Klimaanpassung wurden den Befragten nicht detailliert vorgegeben. Mit einer Ausnahme konnten sich alle Akteure auf diese Interviewoffenheit einstellen. Die Interviews fanden überwiegend in den Geschäftsstellen der befragten Organisationen statt. Sie wurden mitgeschnitten und wörtlich transkribiert. Das auf diese Weise erzeugte Textmaterial umfasste etwa 500 Seiten. Die Auswertung der Interviews wurde mit einem inhaltsanalytischen Verfahren durchgeführt, bei dem eine Kombination aus deduktiver und induktiver Kategorienbildung zur Anwendung kam: Deduktive Kategorien ergaben sich aus den Themenfeldern des Leitfadens, zusätzliche induktive Kategorien entstanden während der Auswertung zu Themen, denen die Befragten im Interviewverlauf eine hohe Relevanz beimaßen.

Ein weiterer sektorübergreifender Workshop (2012) hatte zum Ziel, die Maßnahmen zum Klimaschutz und zur Klimaanpassung, welche in den Interviews und vom Konsortium genannt worden waren, den vier Landnutzungsstrategien von CC-LandStraD (Klimaschutz, Bioenergie, Natur- und Umweltschutz sowie Klimaanpassung) zuzuordnen. Im Anschluss daran wurden die Maßnahmen strategiebezogen in sektoralen Arbeitsgruppen priorisiert und das Ergebnis diskutiert. Hierzu wurden Interviewpartner, Teilnehmer bzw. Eingeladene des Auftaktworkshops und Personen, mit denen das Konsortium in Kontakt stand, sowie einige zusätzliche Stakeholder eingeladen und dabei auf eine relativ ausgewogene Anzahl von Stakeholdern aus den Sektoren Landwirtschaft, Forstwirtschaft, Siedlung und Verkehr sowie Naturschutz geachtet. Erneut wurde vorab ein Informationstext versendet. Dieser umfasste neben einer kurzen Beschreibung der vier Landnutzungsstrategien die Maßnahmen zum Klimaschutz und zur Klimaanpassung. Diese waren sektoral und nach „modellierbar“" und „nicht-modellierbar“ gegliedert. Zusätzlich enthielt die Liste eine kurze Beschreibung jeder Maßnahme sowie gegebenenfalls eine kurze Begründung ihrer Einordnung als nicht-modellierbar.

Nach der Auswahl und Priorisierung der Maßnahmen zum Klimaschutz und zur Klimaanpassung durch das Konsortium und der Stakeholder begannen die Modellierungen für die jeweilige Referenzprojektion der Sektoren Landwirtschaft, Forstwirtschaft sowie Siedlung und Verkehr (vgl. Abschn. 4.1). Aufgrund der fachlichen Spezifika wurden diese Diskussionen in einer sektoralen Workshopreihe geführt. An drei Terminen 2013 und 2014 wurden Inhalte der land- und forstwirtschaftlichen Modellierung sowie der siedlungsbezogenen Fragestellungen mit Interessenvertretern des jeweiligen Sektors diskutiert. Zu den Workshops der Forstwirtschaft sowie der Landwirtschaft wurden zusätzlich naturschutzfachliche Stakeholder eingeladen. Kernpunkt war jeweils die Vorstellung der Modellierungsergebnisse bezogen auf die Referenzprojektion, um die Weichen für die Maßnahmenmodellierung in den vier Strategien zu stellen. Außerdem standen die Ausprägungen einzelner Maßnahmen in den Strategien, ihr Beitrag zum Klimaschutz und zur Klimaanpassung sowie die Ausgestaltung der Strategien zur Diskussion. 
Alle Workshops wurden mittels Audioaufnahmen und eines Ergebnisprotokolls dokumentiert. Letzteres erhielten die Teilnehmer zunächst in der Entwurfsfassung, um Ergänzungen und gegebenenfalls Richtigstellungen von Aussagen zu ermöglichen. Die endgültige Version wurde allen Eingeladenen zugeleitet.

Die tatsächliche Beteiligung an den verschiedenen Workshops variierte teilweise stark (vgl. Tab. 5.8), ohne dass sich die Zahl der eingeladenen Stakeholder aus den einzelnen Sektoren signifikant änderte.

\section{Regionaler Beteiligungsprozess}

In den Fokusregionen wurden leitfadengestützte Experteninterviews, sektorale Fokusgruppendiskussionen und Workshops durchgeführt.

Die leitfadengestützten Experteninterviews zu Beginn der Forschungsarbeiten dienten dem Zweck, die individuelle Problemwahrnehmung und die damit verbundene Einschätzung durch die lokalen Experten zu ermitteln. Außerdem sollten Maßnahmen gesammelt werden, die auf regionaler Ebene dem Klimawandel entgegenwirken. Auch auf regionaler Ebene war das Ziel der Experteninterviews, genauere Einblicke in die Landnutzung, darauf bezogene Konflikte sowie in Klimaschutz- und Klimaanpassungsmaßnahmen zu erhalten (Reed et al. 2009). ${ }^{58}$

In der Altmark wurden den Experten ein Anschreiben, eine Projektdarstellung und ein Interviewleitfaden mit Informationen über das geplante Interview zugesandt. Alle Angeschriebenen waren grundsätzlich zu einem Gespräch bereit. In einem Fall erfolgte ein Verweis an eine höhere behördliche Stelle. In der Rhein-Region wurden den Experten zunächst ein Anschreiben und eine kurze Projektvorstellung geschickt. Nach Terminabsprache erfolgte die Zusendung des Interviewleitfadens. Es gab lediglich eine Absage. Sieben Personen leiteten die Anfrage an aus ihrer Sicht kompetentere Ansprechpartner innerhalb ihrer Organisationen weiter. Die Interviews wurden digital aufgezeichnet und transkribiert, um diese gezielt auswerten zu können.

Im Anschluss an die Interviews wurden sechs sektorale Fokusgruppendiskussionen in den Sektoren Landwirtschaft, Forstwirtschaft sowie Siedlung und Verkehr dazu genutzt, die Interviewergebnisse zu validieren, mögliche Maßnahmen für Klimaschutz und Klimawandel zu diskutieren und weitere, im Einzelgespräch nicht erschließbare Informationen zu gewinnen. Da Fragen des Natur- und Umweltschutzes in den Fokusregionen von erheblicher Bedeutung für die Flächennutzung sind und der Natur- und Umweltschutz damit in Konkurrenz zu den drei genannten Sektoren steht, wurde jeweils eine zusätzliche Fokusgruppendiskussion zu dieser Thematik durchgeführt. Zudem wurden die sektorspezifischen Landnutzungspräferenzen der Interessengruppen diskutiert. In der Diskussion ergänzten die Stakeholder die vom Konsortium vor-

\footnotetext{
${ }^{58}$ Diese Interviews wurden von Wibke Crewett vorbereitet und durchgeführt. Ihre Auswertungen flossen in die nachfolgenden Darstellungen mit ein (vgl. auch Crewett et al. 2013).
} 
geschlagenen Landnutzungsmaßnahmen um weitere Maßnahmen, die aus ihrer Sicht den Klimawandel beeinflussen können. In der qualitativen Sozialforschung hat sich die Fokusgruppe als Methode bewährt (insbesondere in der integrierten partizipativen Bewertung), um Interessen von Stakeholdergruppen zu identifizieren und zu analysieren (Morgan 1996; Krueger und Casey 2000).

Die Planung der Fokusgruppen erfolgte in enger Kooperation mit dem Konsortium, um ein Ineinandergreifen der einzelnen Teilprojekte und Arbeitsschritte sicherzustellen. Außerdem sollten Synergieeffekte erzeugt werden, indem die Projektexpertise in Form von Kurzvorträgen während der Fokusgruppen genutzt wurde und so direkt in die Diskussionen einfließen konnte. Jede Fokusgruppe begann mit einem Einführungsteil, der die Ziele des Projektes und des Stakeholderprozesses vermittelte. Nachfolgend wurden die Bedeutung des Klimawandels in den Fokusregionen sowie anschließend mögliche Landnutzungsmaßnahmen vorgestellt, die dem Klimawandel entgegenwirken können. Die Aufgabe der Teilnehmer war es, zu bewerten, welche Maßnahmen als sinnvoll erachtet werden. Zum Abschluss wurden diese Maßnahmen in ein Ranking eingebracht. Die Fokusgruppengespräche wurden transkribiert und auf Basis der qualitativen Inhaltsanalyse nach Mayring (2010) ausgewertet.

Ab 2014 wurden den Akteuren in vier sektoralen Workshops die Berechnungen der Modellierungen zu Klimaschutz- und Klimaanpassungsmaßnahmen vorgestellt. Die Workshops beschränkten sich auf die Sektoren Landwirtschaft sowie Siedlung und Verkehr, da nur hier Modellierungen für die regionale Ebene erfolgten. Zudem wurde die Flächenwirksamkeit von Klimaschutz- und Klimaanpassungsmaßnahmen diskutiert, um weitere Optionen für die Integration der Maßnahmen in die Modellierung zu eröffnen. Auch Planungs- und weitere Steuerungsinstrumente im Spannungsfeld zwischen Klimawandel und Landnutzung wurden thematisiert. Zur Gewährleistung der Kontinuität des Beteiligungsprozesses wurden in erster Linie die Stakeholder eingeladen, die bereits in der ersten Projektphase involviert waren. So konnte der Anteil an neuen Informationen im Programm hoch gehalten und gleichzeitig die Attraktivität der Workshops gewährleistet werden. Die Einladungen enthielten beispielhaft jeweils ein Modellierungsergebnis, um die Motivation zur Teilnahme weiter zu erhöhen. Die Fokusgruppengespräche wurden mit Hilfe digitaler Audioaufnahmen und in Form eines Ergebnisprotokolls dokumentiert.

Das zahlenmäßige Verhältnis zwischen eingeladenen und tatsächlichen Teilnehmer für die einzelnen Beteiligungsformen im regionalen Beteiligungsprozess ist in Tab. 5.9 dargestellt. Es wird deutlich, dass die Beteiligungsbereitschaft im Zeitverlauf in den Fokusregionen höher war als im nationalen Beteiligungsprozess. Dies könnte darin begründet sein, dass die (unmittelbare) Betroffenheit regional höher ist und dementsprechend eine Beteiligung aus intrinsischen Motiven heraus ausgeprägter war als auf Bundesebene. 


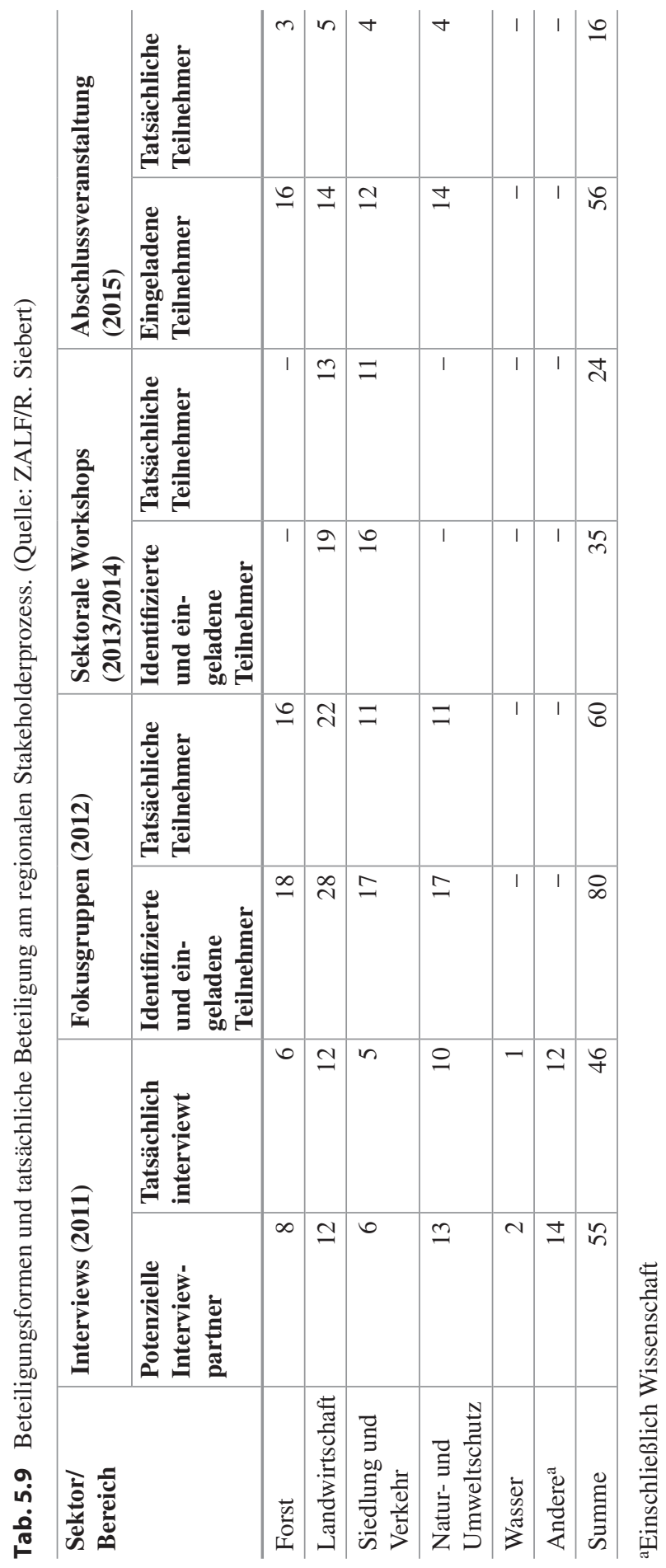




\section{Ebenenübergreifende Aktivitäten}

Eine erste Gesamtschau der Forschungsaktivitäten und -befunde fand 2013 nach zweieinhalb Jahren auf der Zwischenkonferenz statt. Hier wurden Zwischenergebnisse präsentiert und zur Diskussion gestellt, wobei die Veranstaltung einen vorrangig wissenschaftlichen und keinen Workshop-Charakter hatte. Von den eingeladenen 127 Praxispartnern der Bundesebene und aus den Regionen beteiligten sich 23 .

Fünf Monate vor Projektabschluss fand 2015 ein sektorübergreifender Workshop statt, zu dem erstmals die Akteure des bundesweiten und des regionalen Beteiligungsprozesses gemeinsam eingeladen wurden. Der Zeitpunkt war so gewählt, dass wesentliche Ergebnisse der Modellierungen bereits vorlagen, zugleich aber noch die Möglichkeit bestand, die Anmerkungen und Kommentare der Stakeholder in die Endphase des Projektes und somit in mögliche Schlussfolgerungen einfließen zu lassen. Der Fokus dieser Veranstaltung lag, wie von einigen Stakeholdern im Feedback zur Zwischenkonferenz gewünscht, auf der Ergebnisdarstellung unter weitgehender Ausklammerung methodischer Details. Im Vorfeld des Workshops erhielten die Teilnehmer erneut eine Tischvorlage mit einem kompakten Überblick über erste zentrale Forschungsergebnisse. Auf dem Workshop wechselten sich Plenumspräsentationen und sektorale Arbeitsgruppen zur Ergebnisdiskussion ab.

Insgesamt wurden 114 Stakeholder eingeladen, wobei mit 58 bundesweit organisierten und 56 regionalen Stakeholdern eine gleichgewichtige Aufteilung zwischen den verschiedenen Ebenen bestand. Im Vorfeld hatte für die Bundesebene eine weitere Internetrecherche stattgefunden, um noch einmal eine breitere Beteiligung zu ermöglichen. 27 bundesweite und 16 regionale Akteure kamen der Einladung nach. Die geringere Beteiligung regionaler Akteure deutete sich im Vorfeld der Veranstaltung bereits an, da einige potenzielle Teilnehmer die Anreise nach Hannover als zu aufwendig erachteten. Die Fokusregionen liegen geographisch weit voneinander entfernt, deshalb musste ein Kompromiss bei der Auswahl des Veranstaltungsortes eingegangen werden. Die Tab. 5.8 und 5.9 zeigen ein verändertes Beteiligungsmuster an diesem Workshop: Während die bundesweiten Akteure im Vergleich zu den regionalen Praxispartnern bei den vorherigen Workshops in geringerem Maße teilnahmen, waren sie beim sektorübergreifenden Workshop stärker vertreten. ${ }^{59}$

\subsubsection{Feedbackschleifen}

Prozessimmanent war eine Reflexion der dargestellten Aktivitäten. Größere Workshops des bundesweiten und regionalen Beteiligungsprozesses, die Zwischenkonferenz und die Abschlussveranstaltung wurden jeweils mit einem Feedbackbogen beendet. Außerdem erhielten die teilnehmenden Stakeholder stets die Gelegenheit, die Protokollentwürfe der

\footnotetext{
${ }^{59} \mathrm{An}$ den bundesweiten Workshops nahmen darüber hinaus meist auch einzelne Vertreter Nordrhein-Westfalens und Sachsen-Anhalts, also der Bundesländer, in denen die Fallbeispielregionen liegen, teil.
} 
Veranstaltungen zu prüfen und zu kommentieren. Mehrfach wurden Einzelaussagen in den Protokollen daraufhin verändert oder ergänzt.

Von besonderer Bedeutung für den Gesamtprozess war das Feedback der Stakeholder während des sektorübergreifenden Abschlussworkshops - zum einen, weil damit die Gelegenheit für eine Reflexion der sich über viereinhalb Jahre erstreckenden Beteiligungsprozesse gegeben war, zum anderen, weil auch das Konsortium zeitgleich einen ähnlichen Fragebogen erhielt. 19 der 40 auf dem Abschlussworkshop anwesenden Stakeholder füllten einen Feedbackbogen aus. Gefragt wurde unter anderem nach der Praxisrelevanz des Projektes, der Bewertung der methodischen Umsetzung des transdisziplinären Ansatzes, der gesellschaftlichen Bedeutung der Projektergebnisse, der allgemeinen Bewertung transdisziplinärer Zusammenarbeit und Optimierungsmöglichkeiten bei der Zusammenarbeit mit Landnutzern. Aus dem CC-LandStraD-Konsortium beteiligten sich acht Wissenschaftler an der schriftlichen Pendantbefragung und beantworteten den Fragebogen.

Vertiefend wurden 2015 außerdem sieben Schlüsselstakeholder der bundesweiten Ebene - überwiegend solche, die kontinuierlich an den Workshops teilgenommen hatten - telefonisch nochmals intensiver zu ihren Reflexionen des Gesamtprozesses und den letztlich erzielten Ergebnissen im Vergleich zu ihren Erwartungen befragt.

\subsubsection{Stakeholder-Interventionen}

Die Beteiligungsinhalte und die auf den Workshops zur Diskussion gestellten Themenschwerpunkte wurden am jeweils aktuellen Projektstand und den anstehenden Arbeitsschritten ausgerichtet. Aus Sicht des Konsortiums bestanden die Inhalte des transdisziplinären Austauschs vor allem aus:

- der inhaltlichen Ausgestaltung, Interpretation und Benennung von Maßnahmen, Techniken und Instrumenten der Landnutzung (nachfolgend unter der Sammelbezeichnung „Maßnahmen“ zusammengefasst),

- der Zuweisung von Maßnahmen zu Landnutzungsstrategien (nur im bundesweiten Beteiligungsprozess für die Sektoren Landwirtschaft sowie Siedlung und Verkehr) bzw. einer Diskussion der konkreten Maßnahmenausprägungen in den Strategien (nur für die nationale Ebene im Forstsektor) sowie

- einem besseren Verständnis von inter- und intrasektoralen Landnutzungskonflikten.

Als Diskussionsschwerpunkte aus Sicht der Stakeholder sind folgende Themen zu benennen:

- die in der Modellierung berücksichtigten Maßnahmen der Landnutzung und ihre Ausprägungen, 
- die von CC-LandStraD zur Diskussion gestellten Landnutzungsstrategien (nur auf den Workshops auf Bundesebene sowie dem Abschlussworkshop thematisiert) sowie

- übergreifende Aspekte.

Anhand dieser Punkte werden nachfolgend, jeweils nach Sektor und räumlicher Ebene getrennt, beispielhaft einige der Diskussionspunkte und, wenn thematisiert, damit verbundene Landnutzungskonflikte nachvollzogen.

\subsubsection{Sektor Landwirtschaft}

\section{Diskussionspunkte auf bundesweiter Ebene}

Zentrales und im Prozessverlauf immer wieder angesprochenes Thema waren die für die Modellierung berücksichtigten Maßnahmen, ihre Ausgestaltung und Benennung. Als mehrdeutig wurde z. B. die Maßnahme „Erhöhung der Anbaudiversität“ empfunden, die einerseits die Erweiterung der Fruchtfolgen zur besseren Risikostreuung über das Jahr meinte, andererseits aber auch den Anbau alternativer Bioenergiepflanzen (wie Durchwachsene Silphie; Silphium perfoliatum) umfasste. Vielmehr, so einige Stakeholder, sollte jede Maßnahme nur ein Ziel beinhalten. Zugleich wurde angemerkt, dass das Spektrum der Alternativen zum Energiemais breiter als in der Modellierung berücksichtigt sei. Ein anderes Beispiel war die Maßnahme „Wiedervernässung von Mooren“, deren Benennung und Ausrichtung einzelne Stakeholder in den Diskussionen als zu pauschal und nicht überall umsetzbar bezeichneten, da Wiedervernässung auch negative Folgen für die bestehende Flora und Fauna haben könne. Deshalb gehe es eher um „lokale Strategien zur Bewirtschaftung und Entwicklung von Mooren“. Auch seien die Wechselwirkungen einer solchen Maßnahme mit ökonomischen Aspekten (v. a. den Einkommen der Landwirte) genauer zu prüfen und darzustellen.

Die Ausgestaltung der Landnutzungsstrategien war, obwohl ursprünglich vom Konsortium nicht so gedacht, Thema mehrerer Workshops. So kritisierten die Stakeholder unter anderem die inhaltliche Nähe der Strategien untereinander, die sich durch den Projektschwerpunkt auf die Minderung von Treibhausgasen ergab, und regten eine größere Trennschärfe an, auch wenn der Anwendungsbezug dann geringer wäre. Die relativ lange Laufzeit des Projektes führte dazu, dass die Bioenergiestrategie in ihrem Beitrag für den Klimaschutz im Projektverlauf von den Akteuren immer stärker hinterfragt wurde. Zwar war von Anbeginn auf die negativen Auswirkungen der Flächeninanspruchnahme zur Bioenergiegewinnung und die damit verbundenen Landnutzungskonflikte (,Tank oder Teller") hingewiesen worden, doch problematisierten die Stakeholder zunehmend auch die unerwünschten Folgewirkungen und hier insbesondere die steigenden Agrarflächenpreise, die gegen eine Ausweitung der für die Bioenergie genutzten landwirtschaftlichen Flächen sprächen.

Damit im Zusammenhang wurde die Sicherung landwirtschaftlicher Flächen als übergreifendes Landnutzungsproblem aus Sicht der Stakeholder vor allem des Agrarsektors thematisiert. Sie problematisierten immer wieder den Druck auf landwirtschaftliche 
Flächen durch Siedlungs- und Infrastrukturprojekte sowie durch naturschutzfachliche Kompensationsflächen. Dies führe, so die Stakeholder, zu einer stetigen Reduzierung landwirtschaftlicher Fläche. Die Erzeugung erneuerbarer Energien im Rahmen der Energiewende erhöhe den Flächendruck weiter. Um die Ernährungssicherheit zu gewährleisten, seien daher eine effiziente Ressourcennutzung und Flächensicherung notwendig. Gleichzeitig aber seien Verfahren bzw. Maßnahmen zu entwickeln, die auch den wachsenden gesellschaftlichen Ansprüchen an die Landwirtschaft, wie zum Beispiel Nachhaltigkeit und artgerechte Tierhaltung, genauso wie einer effizienten Bewirtschaftung gerecht werden. Hinsichtlich der Projektergebnisse regten einige Akteure an, dass die Klimaschutz- und Klimaanpassungsmaßnahmen nicht nur auf der landwirtschaftlichen Fläche betrachtet werden sollten, sondern auch die Produktionsketten im landwirtschaftlichen Sektor einen entscheidenden Beitrag leisten können.

Schließlich wiesen die Stakeholder wiederholt auf globale Rahmenbedingungen der Agrarproduktion und landwirtschaftlichen Flächennutzung sowie die damit verbundenen Unsicherheiten für eine Modellierung hin, z. B. auf die weltweite Fleischnachfrage, Importe von Futtermitteln oder Auswirkungen nationaler Politiken auf Landnutzungsänderungen in anderen Teilen der Welt.

\section{Diskussionspunkte auf regionaler Ebene}

Im Sektor Landwirtschaft fand eine sehr umfangreiche Maßnahmendiskussion statt. Als bedeutsam wurde die Errichtung von Güllelagern eingestuft. Bei diesem Vorgang kollidieren aus Sicht von Stakeholdern gesetzliche Vorgaben mit den Auffassungen von Politikern und Bürgerinitiativen. Die Akzeptanz in der Bevölkerung für Güllelager sei sehr gering, weil damit intensive Geruchsbelästigung verbunden werde. Die Medien unterstützen nach Meinung zahlreicher Stakeholder die Bevölkerung. Landwirte seien vor einem Bauprojekt gezwungen, „regelrechte Imagekampagnen“ zu starten. Mehr Güllelager bedeuten jedoch aus Sicht der Stakeholder eine effizientere Ausbringung des Wirtschaftsdüngers.

In der Altmark wurden vor allem folgende Aspekte und Maßnahmen diskutiert:

- Der Wasserhaushalt ist ein zentrales Thema in der Region und wurde sowohl von Stakeholdern der Landwirtschaft als auch des Natur- und Umweltschutzes hervorgehoben. Die Beregnung von Ackerflächen ist für Landwirte in der Altmark deshalb eine Maßnahme, die in Zukunft an Bedeutung gewinnen wird. Ebenfalls im Zusammenhang mit der Wasserthematik steht die Wiedervernässung von Mooren zu Ersatz- und Ausgleichszwecken. Die Wiedervernässung bleibt jedoch aus Sicht von Stakeholdern nicht auf ehemalige Moorflächen beschränkt und nimmt weitere landwirtschaftliche Flächen in Anspruch.

- Die Erzeugung und der Einsatz von Bioenergie stellten sich in der Perspektive der Stakeholder als besonders politikabhängig dar. Die sich stetig ändernden Rahmenbedingungen machten eine Nutzung zunehmend unattraktiv. Die Novellierung des EEG hat aus Sicht der Stakeholder den Neubau von Biogasanlagen zum Erliegen 
gebracht. Da aber die bisher gebauten Anlagen eine Bestandsgarantie von 20 Jahren haben, bestehen positive und negative Auswirkungen fort.

- Die Auswahl von Maßnahmen für die Bodenbearbeitung (z. B. Mulchsaat) oder der Düngung sollte nach Auffassung vieler Stakeholder dem Sachverstand des Landwirts überlassen werden. So würde ein Landwirt beispielsweise nicht übermäßig düngen, da dies ökonomisch keinen Sinn ergäbe.

Im Vergleich zur Diskussion in der Altmark lagen die Themenschwerpunkte in der Rhein-Region in deutlich anderen Bereichen:

- Die Extensivierung landwirtschaftlicher Nutzfläche wurde von zahlreichen Stakeholdern abgelehnt, da diese keinen Beitrag zum Klimaschutz leiste.

- Ein Grünlandumbruchverbot wurde aus diesem Grund nicht als zielführend angesehen.

- Der Anbau von Kurzumtriebsplantagen ergibt im Rheinisch-Bergischen Kreis nach Darstellung vieler Stakeholder ebenso keinen Sinn und ist deshalb ihrer Ansicht nach als Klimaschutzmaßnahme uninteressant.

In den Fokusgruppen Umwelt- und Naturschutz wurde zusätzlich über die Maßnahmen Grünlanderhalt und die Neuanlage von Flächen zu Klimaschutzzwecken diskutiert. Die Stakeholder erachteten beides nur mit einer Förderung oder einer ordnungsrechtlichen Verankerung im Rahmen der guten fachlichen Praxis als umsetzbar.

Generell identifizierten die Stakeholder viele Zielkonflikte zwischen Klima-, Arten-, Umwelt- und Landschaftsschutz. In beiden Regionen bewerteten einige von ihnen die Neuausweisung von Schutzgebieten kritisch, da damit Grundeigentum entwertet und die landwirtschaftliche Produktion in der Regel eingeschränkt werde. In der Altmark wurde vor allem auf die Ausweisung neuer Natura 2000-Schutzgebiete in Sachsen-Anhalt verwiesen. Ausgleichs- und Ersatzmaßnahmen wurden ebenfalls kritisch gesehen. Eine Lösung können aus Sicht der Stakeholder produktionsintegrierte Kompensationsmaßnahmen darstellen. In diesem Zusammenhang wurde generell die Nutzung von landwirtschaftlicher Fläche für Ersatz- und Ausgleichsmaßnahmen durch die Teilnehmer kritisiert (z. B. durch den Autobahnbau). Die damit verknüpfte Ökopunkteverordnung bietet aus Sicht zahlreicher Stakeholder in der Altmark sehr viel Diskussionsstoff hinsichtlich der Flächeninanspruchnahme. Ein weiterer Konflikt entsteht in der Rhein-Region durch den Aufkauf landwirtschaftlicher Flächen durch externe Investoren, was zu einer Erhöhung der Pacht- und Kaufpreise führe.

Im Fokus der übergreifenden Diskussionen um den Klimawandel war auffällig, mit welchem globalen Ansatz dieser verbunden wurde. So stellten die Stakeholder die eigene regionale Situation immer wieder in einen weltweiten Zusammenhang. Aus Sicht zahlreicher landwirtschaftlicher Stakeholder würde es vor allem helfen, wenn die Verbraucher von Agrarprodukten ihr Kaufverhalten stärker am Klimaschutz orientierten und sich so die Nachfrage umstelle und regionale Wirtschaftskreisläufe gestärkt würden. 
Stakeholder aus dem Natur- und Umweltschutz in der Rhein-Region betonten, dass THG-Emissionen aus der Landwirtschaft kaum eine Rolle für den Klimawandel spielen und dass die Energiewirtschaft entscheidend für die THG-Bilanz sei. Nicht Klimaschutz, sondern Biodiversität habe in der Region die höchste Priorität. Diese Akteure wünschten sich einen größeren Stellenwert des Themas Biodiversität im Projekt CC-LandStraD.

\subsubsection{Sektor Siedlung und Verkehr}

\section{Diskussionspunkte auf bundesweiter Ebene}

Als wichtigstes Ziel im Siedlungssektor wurde von den Stakeholdern die Reduktion der Flächenneuinanspruchnahme gesehen. Den darauf bezogenen Maßnahmen - Innenentwicklung (mit den Schwerpunkten Wiedernutzung von Brachen, Baulücken und Leerständen), Ausschöpfung baulicher Dichten im Neubau sowie Rückzug aus der Fläche wurde deshalb bei allen Workshops sowie in den Interviews eine wichtige Rolle beigemessen. Die Akteure betonten in diesem Zusammenhang ein gewachsenes Bewusstsein bei den handelnden Praktikern - das sogenannte 30-ha-Ziel sei weithin bekannt -, aber Planungsinstrumente und Negativanreize stünden einer Umsetzung im Wege. Kontrovers diskutiert und für die Modellierung schließlich verworfen wurde eine Maßnahme ,Verteuerung des Bodens“, da diese negative Folgewirkungen für die meisten beteiligten Akteure (Landwirte, Kommunen, private Bauinteressenten) habe. Bezogen auf die Maßnahme „Erhalt und Entwicklung innerstädtischer Freiflächen“ merkte ein Stakeholder an, dass durch zu viel urbanes Grün der städtische Charakter verloren gehen könne. Aus Praxisperspektive wurde betont, dass bei diesem Thema auf kommunaler Ebene bislang sowohl das Problembewusstsein als auch Instrumente und das methodische Rüstzeug fehlten. Als übergreifendes Problem der Maßnahmenausgestaltung thematisierte vor allem ein Vertreter ländlicher Räume, dass Ausprägungen und Auswirkungen der modellierten Maßnahmen in städtischen und ländlichen Räumen sowie in Schrumpfungsund Wachstumsregionen sehr unterschiedlich seien.

Auch an der Diskussion um die Landnutzungsstrategien beteiligten sich die Akteure des Sektors. Allgemein betonten die Stakeholder die Notwendigkeit realistischer Ausprägungen der Maßnahmen für die Strategien (z. B. hinsichtlich des tatsächlichen Versiegelungsgrades wiedergenutzter Brachflächen). Auf einem Workshop wurde zusätzlich zu den vier betrachteten Landnutzungsstrategien eine weitere Strategie „Flächensicherung“ vorgeschlagen - bezogen auf den Schutz agrarischer Flächen. Denn Flächenumwidmungen für Siedlungszwecke erfolgen vorrangig zu Lasten agrarischer Flächen. Hier wandte ein Stakeholder des Sektors Siedlung und Verkehr ein, dass die Bevorzugung einer Flächennutzung vor allen anderen aus seiner Sicht nicht gerechtfertigt sei. Vielmehr gäbe es legitime Flächennutzungsinteressen auch der nicht-agrarischen Sektoren. Zudem verwiesen die Flächendaten und -nachfrageprojektionen des BBSR auf große Veränderungen im Bereich der Siedlungs- und Freiflächen. Die Zahl der Strategien wurde im Nachgang dieses Workshops nicht verändert, aber die Thematik der Reduzierung des Flächenverbrauchs gewann weiter an Bedeutung. Schließlich wurden 
Zielkonflikte zwischen den Strategien Klimaschutz und Klimaanpassung thematisiert: Auch bei einem Schwerpunkt auf der Innenentwicklung sei zu beachten, dass Freiflächen zur Naherholung und notwendige Frischluftschneisen zur Belüftung gesichert werden.

In übergreifender Perspektive war es den Stakeholdern dieses Sektors einerseits wichtig, Klimawandel nicht auf die Reduktion von Treibhausgasen zu beschränken vielmehr wurden auch die negativen Auswirkungen des Klimawandels, vor allem die zu erwartende Zunahme von Extremereignissen (insbesondere Hochwasser), thematisiert. Andererseits betteten sie Fragen des Klimaschutzes als Forschungsschwerpunkt des Projektes in weitere gesellschaftliche Entwicklungen - wie den demographischen Wandel, die Frage regionaler Disparitäten oder die Notwendigkeit (und Grenzen) interkommunaler Kooperationen - ein.

\section{Diskussionspunkte auf regionaler Ebene}

Die Maßnahmendiskussion in der Altmark wurde von dem die Region seit langem prägenden Thema „Demographischer Wandel“ (vor allem durch Abwanderung) und die so verursachten veränderten Siedlungsstrukturen und Immobilienleerstände überlagert. Die insbesondere mit dem Städtebauförderprogramm „Stadtumbau Ost“ verbundene Förderung einer kompakten Siedlungsstruktur begünstigt nach Ansicht der Stakeholder gleichzeitig den Klimaschutz und die Innenentwicklung. Die Maßnahme „Erhalt von Freiflächen“ spiele demgegenüber in der Region keine Rolle, sondern eher der Umgang mit Brachen. Durch zahlreiche Stakeholder wurde kritisiert, dass Entsiegelungsmaßnahmen nicht für Ersatz und Ausgleich herangezogen werden, weil die Kosten dafür zu hoch sind. Dabei könnten ihrer Meinung nach landwirtschaftliche Flächen so geschont werden. Brachenentsiegelung und Lösung des Leerstandsproblems wurden als drängende Fragen thematisiert. Insgesamt stellten zahlreiche Stakeholder den Sinn von Ausgleichsmaßnahmen in Frage, da die Flächen oft nicht betreut und überwacht würden. Die Nutzung erneuerbarer Energien auf Siedlungsflächen ist aus Sicht der Stakeholder ein sinnvoller Weg, die THG-Emissionen zu verringern. Gleichzeitig wurde betont, dass speziell die Förderung der Bioenergie Verwerfungen im landwirtschaftlichen Flächenmarkt verursache. Schließlich betonten die Stakeholder aus der Altmark, dass bei der Landnutzung Prioritäten gesetzt werden müssten. Dies bezog sich auf die für die Region ebenfalls relevante Frage des Hochwasserschutzes: Siedlungsentwicklung führe zur Expansion in der Fläche, Hochwasserschutz fordere hingegen eine Konzentration der Bevölkerung.

In der Rhein-Region wurde die Maßnahmendiskussion bei unterschiedlicher Ausgangslage (hohe Flächennachfrage durch wirtschaftliches Wachstum und positives Wanderungssaldo) von ähnlichen Argumenten geleitet. Den Stakeholdern zufolge wird aus demographischen Gründen - also nicht aufgrund des Klimawandels - bereits auf eine Nachverdichtung hingearbeitet. Außerdem kollidiere die Nachverdichtung mit anderen Klimamaßnahmen: So sei eine Erhöhung des Grünflächenanteils damit nur schwer zu vereinbaren. Zudem wünschen sich viele potenzielle Zuzügler ein Einfamilienhaus. Weiterhin wird das zu erwartende Hitzeproblem mit einer Nachver- 
dichtung aus Sicht der Stakeholder verschärft. Hier gibt es ihrer Meinung nach einen Zielkonflikt zwischen Klimaschutz und Klimaanpassung. Baulückenkataster existieren bereits, um die Nachverdichtung zu unterstützen, aber diese helfen nicht immer, da die Grundstücke sehr oft aus ganz individuellen Gründen leerstehen. Das 30-ha-Ziel verschärfe die Flächenknappheit für Wohnbauland noch und ist deshalb aus Sicht einiger Stakeholder nicht sinnvoll. Die Konkurrenz um Flächen wird zudem zwischen Kommunen ausgetragen, die mit allen Mitteln versuchen, für Gewerbetreibende attraktiv zu sein. Klimaauflagen, wie begrünte Dachflächen, seien deshalb kaum durchsetzbar. Die Gebäudesanierung als Klimaschutzbeitrag wurde infrage gestellt. Hier wäre es aus Sicht der Stakeholder interessant, die THG-Emissionen für die Sanierung den Einsparungen gegenüberzustellen. Generell war in der Rhein-Region ein Umsetzungswille für die Maßnahmen vorhanden, aber ihre globale Wirkung wurde angezweifelt.

Übergreifende Landnutzungskonflikte wurden in der Rhein-Region insbesondere in Bezug auf Flächenverbrauch und Flächenknappheit sowie im Zusammenhang mit Ausgleichs- und Ersatzmaßnahmen thematisiert. In der Altmark spielen diese vor allem im Zusammenhang mit dem Ausbau der Bundesautobahn 14 eine Rolle. Die Stakeholder favorisierten für die Umsetzung von Ausgleichs- und Ersatzmaßnahmen die Entsiegelung von Industriebrachen oder produktionsintegrierte Maßnahmen. Auch der Ausbau der Bioenergie wurde problematisiert. Einerseits hielten die Stakeholder die Nutzung erneuerbarer Energien in den Siedlungen für einen geeigneten Weg, um den THGAusstoß zu reduzieren, andererseits betonten sie, dass die Bevölkerung der Ansiedlung von Biogasanlagen im Wohnumfeld negativ gegenüberstehe, da ihre Lebensqualität durch die Errichtung negativ beeinflusst werde.

\subsubsection{Sektor Forstwirtschaft}

\section{Diskussionspunkte auf bundesweiter Ebene}

Anders als in den Sektoren Landwirtschaft sowie Siedlung und Verkehr wurden auf den Workshops mit den Forstvertretern keine Einzelmaßnahmen unabhängig von der jeweils verfolgten Landnutzungsstrategie diskutiert. Vielmehr besteht jede der vier Strategien aus unterschiedlichen Ausprägungen von jeweils elf Maßnahmen. Jede Strategie ist somit zugleich ein Maßnahmenbündel (vgl. Abschn. 3.3.6). Die Maßnahmen waren erstmals in den Experteninterviews 2011/2012 erhoben worden, wurden anschließend mit denen der Wissenschaftler abgeglichen und soweit wie möglich in der weiteren Modellierung berücksichtigt. Ein Workshop wurde deshalb nur darauf verwendet, die von den Fachwissenschaftlern vorgeschlagenen Maßnahmenausprägungen in den drei Strategien Klimaschutz, Bioenergie, Natur- und Umweltschutz zu prüfen und gegebenenfalls Änderungsvorschläge zu äußern (die Klimaanpassungsstrategie im Forst wurde erst nachträglich entwickelt). Die Stakeholder ließen sich auf dieses Vorgehen ein, betonten aber, dass die Maßnahmen nicht nur je nach Ausprägung, sondern auch in Abhängigkeit von den spezifischen Standorteigenschaften unterschiedlich wirken, und dies bei der Interpretation der Modellierungsergebnisse zu berücksichtigen sei. 
Bezogen auf die Strategien gab es unter anderem Kritik an der Bezeichnung der Strategie „Natur- und Umweltschutz“. Es wurde angemerkt, dass Naturschutz in der Forstwirtschaft nicht gleichzusetzen sei mit Umweltschutz - Naturschutz bedeute vielmehr Artenschutz, der in der Strategie nicht mit abgebildet werde. Auch über die Ziele der Strategien wurde diskutiert: So wiesen die Stakeholder für die Klimaschutzstrategie darauf hin, dass die Erhöhung des Zuwachses das entscheidende Kriterium sei und der Vorratsaufbau als zweitrangig angesehen werde. Die Beschreibung des Ziels wurde daraufhin angepasst. Ein Teil der Diskussion drehte sich des Weiteren darum, ob nicht auch „Extremstrategien“, wie z. B. $80 \%$ Douglasie, modelliert werden könnten. Diese Anregung wurde vom Konsortium mit dem Verweis auf die durch das Projekt auch zu leistende glaubwürdige Politikberatung zurückgewiesen - eine solch extreme Maßnahmenausprägung sei demnach nicht vermittelbar. Weitere Hinweise bezogen sich auf die Baumartenwahl und die Anteile einzelner Baumarten.

Übergreifend diskutierten die nationalen Stakeholder im Sektor Forst so stark wie in keinem anderen Sektor über die Modellierung und ihre Grenzen. Dies war auch dem Umstand geschuldet, dass das genutzte Modell FoBeSiMo (vgl. Abschn. 4.2.2.4) neu entwickelt worden war. So wünschten sich die Stakeholder beispielsweise bestimmte Maßnahmen (z. B. eine verbesserte Ausschöpfung der Holzpotenziale im Kleinstprivatwald), die aus Sicht der Praxis von hoher Relevanz seien, aber in der Modellierung aufgrund unzureichender Daten nicht berücksichtigt werden konnten. Auch eine stärkere Regionalisierung wurde angemahnt, konnte jedoch wegen fehlender Datengrundlagen und aus Kapazitätsgründen - ebenso wie der Einbezug weiterer Maßnahmen oder Baumarten - in diesem Projekt nicht geleistet werden. Mehrfach wiesen die Stakeholder deshalb darauf hin, dass die Einschränkungen (einschließlich des begrenzten Modellierungszeitraums von 40 Jahren) bei Ergebnisvorstellungen und -interpretationen deutlich gemacht werden müssten, da sonst der Eindruck zu pauschaler Aussagen entstehen könne.

\section{Diskussionspunkte auf regionaler Ebene}

Die Entwicklung des Modells FoBeSiMo im Projektverlauf und die nur auf Bundeslandebene mögliche Regionalisierung des Modells machte seine Anwendung auf die Fokusregionen nicht möglich. Aus diesem Grund wurde auf regionaler Ebene nur in der ersten Projektphase im Rahmen von Fokusgruppen über die in den Experteninterviews gesammelten Maßnahmen zu Klimaschutz und Klimaanpassung sowie mögliche weitere Maßnahmen diskutiert. Diese bezogen sich vor allem auf die Baumartenwahl im Zusammenhang mit dem Klimawandel und die Holznachfrage. Ein wichtiger Aspekt war ein klimaangepasster Waldumbau. Die Akteure in den Fokusregionen waren der Meinung, dass heimische Baumarten keine Antwort auf den Klimawandel seien. „Standortheimisch“ wurde durch Teilnehmer der Fokusgruppen als ,ideologisch“ bezeichnet, da sich die Standortbedingungen über die Zeit wandeln. „Standortpotenzialgerecht“ wurde als alternativer Begriff vorgeschlagen. Neue Baumarten könnten nach Aussagen von Stakeholdern der Atmosphäre deutlich mehr Kohlendioxid entziehen als heimische. 
Außerdem können Schädlinge, die durch den Klimawandel begünstigt werden, ebenfalls durch die Nutzung neuer Baumarten zurückgedrängt werden. Die Alternative zur Schädlingsbekämpfung wäre nur die Neuzulassung von Insektiziden, und diese sei zu langwierig. Die notwendigen Bearbeitungszeiträume für die Neuzulassung lassen aus Sicht der Stakeholder die Zerstörung großer Waldflächen zu. Dies könne wiederum zu einer Störung des Mikroklimas führen, was Nebelbildung, stärkere Winde und Sandverwehungen nach sich ziehen könnte. Ferner wurde in beiden Regionen betont, dass Holz als Kohlenstoffspeicher nur effektiv zu nutzen sei, wenn eine Kaskadennutzung realisiert werde.

Des Weiteren gab es eine Reihe von unterschiedlichen Problemlagen in den Regionen. So wurde in der Fokusgruppe in der Region Rhein argumentiert, dass ein Waldumbau hin zu Laubgehölzen die Nachfrage nach Nadelholz missachte. Hinzu kommt, dass die Vielzahl von kleinen Waldbesitzern aus Perspektive einiger Stakeholder die ökologische Stabilität des Waldes fördere, da jeder Besitzer eine andere Strategie der Pflege verfolge und so ein Muster von vielen verschiedenen Baumarten entsteht. Regulierungsmaßnahmen vonseiten des Gesetzgebers störten nach Ansicht einiger Stakeholder diese Stabilität. Als Folge des Klimawandels wurde beschrieben, dass der Waldbestand durch Schneebruch gefährdet sei, wodurch vermehrt Schadholz anfalle.

Die Ergebnisvorstellung der Strategiemodellierung auf dem Abschlussworkshop führte zu Diskussionen über eine wünschenswerte Regionalisierung der Forschungsergebnisse. Die Stakeholder kommentierten die Aussagen für die Bundesebene mit ihren Kenntnissen über regionale Spezifika und verwiesen auf die Bedeutung des Projektes für die Politikberatung - zu pauschale Aussagen könnten falsche Schlüsse der Politik (etwa bezogen auf eine Ausweitung des Buchenbestandes) nach sich ziehen.

\subsubsection{Wissenschaft und Praxis in Interaktion: Prozessreflexionen}

Knapp fünf Jahre transdisziplinärer Diskurs mit einer Vielzahl von Einzelaktivitäten sollen in diesem Abschnitt einerseits aus Sicht der Stakeholder, andererseits aus der Perspektive des Forschungskonsortiums reflektiert werden. Dafür wird schwerpunktmäßig auf das Material aus den Experteninterviews sowie den Feedbackschleifen zurückgegriffen.

\subsubsection{Der transdisziplinäre Diskurs aus Sicht der Stakeholder}

\section{Erwartungen an das Forschungsvorhaben}

$\mathrm{Zu}$ Projektbeginn wurden in Interviews auch die Erwartungen der Stakeholder an das Forschungsprojekt erfragt. Diese lassen sich für die bundesweiten Akteure mit vier Stichworten zusammenfassen: 
- Komplexitätsreduktion: Landnutzung und Klimawandel wurden als zwei umfassende Themen wahrgenommen, für die sich die Akteure eine Aufarbeitung bestehenden Wissens und seine Bewertung, aber auch möglichst kleinräumige Projektionen, vor allem in Bezug auf den Klimawandel, wünschten.

- Wissenschaftliche Entscheidungshilfe: Die Akteure erhofften sich von der Wissenschaft den notwenigen Input, um eigene Landnutzungsentscheidungen legitimieren oder politische Entscheidungen begründen zu können.

- Handlungsempfehlungen: Das Projekt solle möglichst konkrete Handlungsempfehlungen erarbeiten, bezogen darauf, was Landnutzer für Klimaschutz und Klimaanpassung tun können. Allgemeine Empfehlungen seien nicht ausreichend.

- Vernetzung von Wissensbeständen und Akteuren: Durch den interdisziplinären Forschungsverbund und den transdisziplinären Ansatz erhofften sich die Stakeholder zum einen ein Zusammenkommen unterschiedlicher Akteure, um den Wissensaustausch zu fördern. Zum anderen solle Deutschland nicht allein gesehen, sondern die Forschung müsse in globale Trends eingebettet werden. Schließlich sollten Erkenntnisse früherer Studien und bestehender Forschungen berücksichtigt werden, um auch als Forschung wirklich nachhaltig zu sein.

Auch die regionalen Stakeholder äußerten in zweierlei Hinsicht Erwartungen an das Projekt:

- Entscheidungsunterstützung: Die Erwartungen der Stakeholder richteten sich in erster Linie an mehr Wissen, z. B. bezogen auf regionale Klimadaten, den Beitrag unterschiedlicher Landnutzungssektoren zum Klimawandel sowie das Potenzial sektoraler Anstrengungen und konkreter Maßnahmen zur Senkung von Treibhausgasen und zur Anpassung an die Folgen des Klimawandels. Auch das Wechselspiel der unterschiedlichen Maßnahmen war von Interesse - je detaillierter, desto besser.

- Netzwerkbildung: Die Vernetzung der Akteure sahen viele Akteure als Mehrwert eines transdisziplinären Ansatzes. Von Interesse war für sie auch, über die eigene Region hinauszuschauen und die Probleme und Lösungsansätze der jeweils anderen Fokusregion kennenzulernen.

Neben der Forderung, dass Forschung möglichst konkrete Empfehlungen geben sollte, äußerten die bundesweiten und regionalen Stakeholder - in den Interviews, aber auch auf den Workshops und in den Fokusgruppen im weiteren Prozessverlauf - die Erwartung, nicht nur neues Wissen zu erhalten, sondern bestehende Erkenntnisse einzubetten, möglichst konkrete Ergebnisse zu erzielen und sich mit anderen Akteuren, gerade im übersektoralen Kontext, auszutauschen.

\section{Rückschau am Projektende}

Auf dem Abschlussworkshop wurden die teilnehmenden Stakeholder mittels eines Feedbackbogens befragt, wie sie die Methode der Workshops während des trans- 
Tab. 5.10 Strukturdaten der feedbackgebenden Stakeholder $(n=19)$. (Quelle: Thünen-Institut/A. Steinführer)

\begin{tabular}{l|l|l|l|l|l|l|l}
\hline \multicolumn{2}{l|}{ Teilnahme } & \multicolumn{2}{l}{ Sektoren } & \multicolumn{2}{l|}{ Institution } & \multicolumn{2}{l}{ Geschlecht } \\
\hline $\begin{array}{l}\text { Erstmals teil- } \\
\text { genommen }\end{array}$ & 9 & Landwirtschaft & $9^{\mathrm{a}}$ & Verwaltung & 10 & Männlich & 14 \\
\hline $\begin{array}{l}\text { Zweimal teil- } \\
\text { genommen }\end{array}$ & 3 & Forstwirtschaft & 1 & $\begin{array}{l}\text { Nichtstaatliche } \\
\text { Organisationen }\end{array}$ & 3 & Weiblich & 5 \\
\hline $\begin{array}{l}\text { Mehr als zweimal } \\
\text { teilgenommen }\end{array}$ & 7 & Siedlung & 6 & Privatwirtschaft & 2 & & \\
\cline { 2 - 5 } & $\begin{array}{l}\text { Natur- und } \\
\text { Umweltschutz }\end{array}$ & $6^{\text {a }}$ & Sonstige & 4 & & \\
\hline
\end{tabular}

${ }^{a}$ Drei Akteure gaben sowohl Landwirtschaft als auch Naturschutz an und werden deshalb zu beiden Sektoren gezählt

disziplinären Diskurses von CC-LandStraD bewerten, wie sie den Mehrwert insgesamt und für ihre Arbeit einschätzen und welche Verbesserungspotenziale sie sehen. Einige Strukturdaten der 19 Akteure, die einen Fragebogen ausfüllten, fasst Tab. 5.10 zusammen. Dass knapp die Hälfte von ihnen erstmals an einem Workshop teilgenommen hatte, war angesichts der Langfristigkeit des transdisziplinären Diskurses nicht zu erwarten gewesen. Unter diesen neun Stakeholdern befinden sich aber sowohl Akteure, die als Experten im ersten Teil des Projektes persönlich befragt worden waren, als auch Vertreter von Organisationen, die an vorherigen Workshops teilgenommen hatten, bei denen sich aber die Personalsituation im Projektverlauf verändert hatte.

Der überwiegende Teil der Stakeholder (17) hielt die durchgeführten Workshops für geeignet, um Wissen und Beiträge aus der wissenschaftlichen Arbeit und der Praxis miteinander zu verbinden. Ebenso viele Stakeholder (17) sahen einen Zusatznutzen durch eine enge Zusammenarbeit von Wissenschaftlern und Praktikern in solchen Projekten gegenüber anderen Forschungsansätzen. Der Transfer in beiden Bereichen wurde als wichtig erachtet und sollte noch intensiviert werden. Dabei gelte es, darauf zu achten, dass sich das Verständnis für die Arbeitsweisen gegenseitig verbessert. Problemstellungen, Folgen und Wirkungen werden zum Teil unterschiedlich bearbeitet und begründet. Der Austausch zwischen Wissenschaft und Praxis trägt aus Sicht eines Akteurs zu einer sachlichen Diskussion und Bewertung von Lösungen bei. Der Austausch von Praxis, Forschung und Politik war aus Sicht der Stakeholder zwingend erforderlich, denn dadurch konnten Maßnahmen, die dann in der Praxis und konkret vor Ort umgesetzt werden sollten, besprochen und festgelegt werden. Durch diesen Rückkopplungsprozess könnten die Wissenschaftler erleben und erfahren, wo es in der Praxis „brennt“. In diesem Prozess sollte die Praxis „Stichwortgeber und Korrektiv“ sein, sie sollte aber den Lösungsraum auch nicht zu sehr einengen, um der Forschung entsprechend Raum zu geben. Ein Akteur kommentierte das wie folgt: „,Sachzwang “ und ,Alternativlosigkeit‘ sind Gift für die ,freie Forschung“." Ein weiteres Argument war, dass die Zusammenarbeit zwischen Wissenschaftlern und Praktikern einer Korrektur 
von falschen Annahmen aus Sicht der Wissenschaft dienen kann und durch den Austausch gegebenenfalls korrigiert und geändert werden kann. Ein Akteur schätzte ein, dass Praktiker von Ergebnissen durch eine Zusammenarbeit sogar stärker profitieren.

Es gab nur wenige Stakeholder, die den Feedbackbogen ausfüllten, die kaum oder keinen Mehrwert durch diese Art der Zusammenarbeit gegenüber anderen Projekten sahen. Begründet wurde das nur durch einen Stakeholder. Sein Argument gegen diese Art von Projekten war, dass aus seiner Sicht nur wenige Lösungsansätze in der Praxis anwendbar seien.

Alle befragten Stakeholder gaben an, dass das Forschungsprojekt mit den Themen Klimawandel und Landnutzung praxisrelevante Problemstellungen aufgegriffen hat (davon 12 mit „eher ja“). Ein Stakeholder stellte dies explizit heraus und bezeichnete die Bedeutung der Landnutzung für den Klimaschutz und die Anpassung als sehr hoch und praxisrelevant. Positiv wurde der sektorübergreifende Ansatz hervorgehoben. Darüber hinaus wurde positiv bewertet, dass sich das Projekt mit den „Schnittstellen zwischen Naturschutz, Landwirtschaft und Treibhausgasen“ beschäftigt hat. Nach Meinung eines Stakeholders konnten praxisrelevante Fragestellungen in die Diskussion eingebracht werden, die im Ergebnis ,eine umfassende Sammlung und Übersicht [...] für Praxis und Wissenschaft" ergaben (gemeint war vermutlich die Maßnahmensammlung; Anm. d. Verf.). Die Diskussion zu Ursachen, Wirkungen und Folgen über die Maßnahmen habe zu einer sachlichen und ausgewogenen Betrachtung geführt. Flächenverbrauch, Landwirtschaft und Naturschutz, so gab ein Stakeholder an, hatte er bisher nicht so stark unter Klimaaspekten betrachtet, will das aber nun stärker in seine fachliche Praxis integrieren. Die behandelten Themen waren aus Sicht eines weiteren Praxisakteurs sehr komplex und aktuell, allerdings gäbe es für vieles oft keine einfachen Lösungen. Kritische Anmerkungen bezogen sich vor allem auf die Praxisrelevanz: So bedauerte ein Stakeholder, dass nicht alle praxisrelevanten Einflussfaktoren (z. B. abiotische und biotische Faktoren vor dem Hintergrund des Klimawandels) in ausreichendem Umfang berücksichtigt werden konnten. Ein anderer Akteur kritisierte, dass Modelle, die nur eine Projektion über 40 Jahre vornehmen, nicht praxisbezogen seien. Ein weiterer Stakeholder hatte sich eine stärkere Auswertung der Ergebnisse bis zur lokalen Ebene gewünscht.

Auf die hypothetische Frage, ob bei einem Neustart des Projektes in der Zusammenarbeit mit den Akteuren etwas anders gemacht werden sollte, gab es zahlreiche Vorschläge, z. B. Methoden des Gruppencoachings zu nutzen, Praktiker noch stärker einzubinden und eine engere Terminabfolge für den Austausch von Wissenschaftlern und Praktikern zu planen sowie lokale Workshops durchzuführen, um die Erfahrungen der Praxis noch stärker in die Ergebnisse einfließen zu lassen. Auch gab es Vorstellungen, statt einer bundesweiten Modellierung besser nur ausgewählte Regionen zu modellieren. Einige Stakeholder wünschten sich noch mehr Zeit für Diskussionen der Ergebnisse. Ein Akteur schlug vor, weitere Stakeholder (z. B. Vertreter des Gesundheitsbereichs) einzubinden, um auch der Frage nachzugehen, ob durch eine geänderte Landnutzung mög- 
licherweise Gefahren durch Krankheitserreger, die längst ausgerottet waren, wieder Einzug finden könnten.

Eine weitere Möglichkeit für ein vertieftes Prozess- und Projektfeedback boten sieben Telefoninterviews mit Stakeholdern der Bundesebene sowie einem Ländervertreter nach dem Abschlussworkshop 2015. Deren Ergebnisse werden nachfolgend zusammengefasst.

In Bezug auf die Motivation zur Teilnahme am transdisziplinären Diskurs wurde von den Stakeholdern in diesen Interviews darauf hingewiesen, dass ihre Institution den Besuch wissenschaftlicher Veranstaltungen als eine Aufgabe ihrer Arbeit sehe, um dort die eigenen Interessen zu vertreten. Ein Akteur fügte hinzu, dass sich seine Organisation als „Knotenpunkt“ zwischen Wissenschaft und Praxis verstehe und durch eine Teilnahme Erkenntnisse in die Praxis transportiert werden könnten. Ein anderer Stakeholder argumentierte in die Gegenrichtung: Forderungen an die Politik sollten seines Erachtens auf wissenschaftlichen Erkenntnissen beruhen, deshalb diene seine Teilnahme der laufenden Informationsgewinnung über aktuelle Forschungsdiskussionen.

Die Mehrzahl der Akteure hob den sektorübergreifenden Ansatz des Forschungsprojekts positiv hervor. Diese ganzheitliche Betrachtung habe es bisher noch nicht gegeben und weckte die Neugier der Stakeholder. Die Erwartungen der befragten Akteure an das Projekt sind auf unterschiedlichen Ebenen anzusiedeln. Einige Stakeholder hatten eine eher allgemeine Erwartungshaltung, die sich vor allem auf die sektorübergreifenden Diskussionen bezog. Diese Akteure bejahten die Frage, ob ihre Erwartungen erfüllt worden seien. Akteure der Landnutzung zusammenzubringen und ,sachlich“ zu diskutieren sowie die aktuelle Diskussion zum Klimawandel aufzubereiten, wurde ebenfalls positiv bewertet. Diese Befragten erwarteten aufgrund der Anlage des Gesamtprojekts im Ergebnis keine „konkrete Hilfestellung“ im Sinne spezieller Landnutzungsmaßnahmen. Ein Stakeholder merkte an, dass zu pauschale Ergebnisse zu falschen Schlüssen für die Politik führen könnten. Darüber hinaus sah er durch den fehlenden Regionsansatz in der Forstmodellierung keine Praxisrelevanz der erzielten Ergebnisse. Andere Forschungsprojekte haben seiner Meinung nach hierzu bereits mehr Erkenntnisse gewonnen und seien für die Praxis von größerer Bedeutung. Ein anderer Akteur bedauerte, dass in der Landwirtschaft die Nutzung von Alternativpflanzen bei Bioenergiepflanzen zwar mitbedacht wurde, aber in der Diskussion zunehmend in den Hintergrund getreten ist. Auch Aspekte des Boden- und Grünlandschutzes zur Sicherung der Biodiversität seien seiner Meinung nach nicht hinreichend berücksichtigt worden. Von einem Stakeholder wurden die Diskussionsrunden, besonders in der dritten Workshopschleife, als zu klein empfunden. Auch sei so „mehr über die Praxis, theoretisch über die Praxis gesprochen [worden], als sozusagen mit der Praxis“.

Fast alle befragten Stakeholder waren der Meinung, dass ihre Anmerkungen und Anregungen durch das Projektkonsortium soweit wie möglich berücksichtigt worden sind. Ein Befragter wies darauf hin, dass er immer auf ein „offenes Ohr“ gestoßen ist. Ein Stakeholder bedauerte hingegen, dass Anmerkungen zwar diskutiert worden seien, aber häufig nicht weiter berücksichtigt werden konnten. Grundsätzlich waren die Erklärungen zu Modellgrenzen für alle befragten Akteure nachvollziehbar und die 
Begründungen, warum Aspekte nicht aufgegriffen werden konnten, einleuchtend. Einige der Befragten kritisierten jedoch, dass es grundsätzlich problematisch sei, die Thematik der zukünftigen Landnutzung vor dem Hintergrund des Klimawandels mit Modellierungen anzugehen. Das Problem sei zu komplex und zu undurchschaubar, als dass Modelle die Realität abbilden oder hilfreiche Ergebnisse für eine zukünftige Strategie liefern könnten. Ein anderer Stakeholder merkte an, dass Modellierungen an sich hilfreiche Instrumente zur Bearbeitung einer Problemstellung sein können, doch in CC-LandStraD seien seine Erwartungen nicht in jeder Hinsicht erfüllt worden.

Einen Zusatznutzen für die eigene Arbeit sieht die Mehrzahl der befragten Akteure. Nach Einschätzung zweier Befragter benötigen von Verbänden und anderen Organisationen formulierte Forderungen auch eine wissenschaftliche Basis. Daher sei die Beteiligung an einem solchen Forschungsprojekt eine Chance, in den Diskurs mit der Wissenschaft zu kommen und über den neuesten Forschungsstand informiert zu werden. Ein anderer Akteur merkte an, dass er seinen Wissenshorizont durch die Diskussionen erweitern konnte. Nur für einen Befragten erbrachte die Projektbeteiligung keinen Zusatznutzen für seine Arbeit. Durch den aus seiner Sicht ungenügenden regionalen Bezug im Projekt fehlten konkrete Anhaltspunkte für die Praxis.

Fast alle Befragten würden sich noch einmal an diesem oder einem ähnlichen Forschungsprojekt beteiligen. CC-LandStraD hat aus Sicht der Stakeholder ein relevantes und aktuelles Thema aufgegriffen und gab ihnen die Möglichkeit, ,über den Tellerrand“ zu schauen. Fast alle Befragten wünschten sich, dass sie die Interessen der eigenen Institution bereits in einem frühen Forschungsstadium einfließen lassen könnten, um den Projektverlauf von Anbeginn mitzugestalten. Das würde für alle Akteure eine wichtige Motivation für eine erneute Teilnahme darstellen. Lediglich ein Stakeholder wies darauf hin, dass er im Vorfeld eines neuen Projektes die methodische Herangehensweise genauer prüfen und sich nur dann erneut beteiligen würde, wenn dieses ,tiefer in die Region, in die Differenzierung“ geht und somit ein „praktischer Nutzen“ gewährleistet wäre.

\subsubsection{Der transdisziplinäre Diskurs aus Sicht des Konsortiums}

Auch die Mitglieder des CC-LandStraD-Konsortiums wurden mittels eines Feedbackbogens befragt, wie sie die Zusammenarbeit mit den Stakeholdern im Projekt bewerten. Von den zehn Konsortiumswissenschaftlern, die aktiv in den Beteiligungsprozess eingebunden waren, beteiligten sich an der Befragung acht. Alle Wissenschaftler, die den Feedbackbogen ausfüllten, hatten an Veranstaltungen des bundesweiten und/oder regionalen Beteiligungsprozesses teilgenommen, wenn auch in unterschiedlicher Intensität. Die Nennungen reichten von einer Veranstaltung $(n=1)$, über drei $(3)$, sechs $(2)$, sieben (1) bis hin zu neun (1).

Die überwiegende Zahl der Wissenschaftler schätzte die Workshops im Rahmen des bundesweiten und regionalen Beteiligungsprozesses als geeignete Methode ein, um Wissensbeiträge aus der wissenschaftlichen Arbeit und der Praxis miteinander zu verbinden. Ein Forscher schlug das Planspiel als eine gute Methode in Ergänzung zu den 
Workshops vor, um einen noch aktiveren Beitrag der Stakeholder zu ermöglichen. Allerdings schränkte ein anderer Wissenschaftler auf die Frage nach weiteren möglichen Methoden ein, dass die zur Verfügung stehenden Mittel auch Grenzen der Beteiligung setzten. Die meisten befragten Fachwissenschaftler hatten über die bundesweiten und regionalen Workshops hinaus Kontakte mit den in den Projektprozess eingebundenen Stakeholdern bezüglich CC-LandStraD-spezifischer Fragen. Für fünf Wissenschaftler waren die Anregungen, Kritikpunkte und Fragen der Stakeholder zu den Zwischenergebnissen des Projektes für das Gesamtprojekt „,(eher) hilfreich“, für einen Befragten ,,sehr hilfreich“ und für zwei Wissenschaftler „,eher wenig hilfreich“. Für die eigene Arbeit erachteten fünf Mitglieder des Konsortiums die Anregungen der Stakeholder als „,eher hilfreich“, drei als „eher wenig hilfreich“.

Positiv bewerteten einige Wissenschaftler, dass die Forschungsergebnisse durch den Austausch mit den Akteuren an den Bedürfnissen der Praxis gemessen wurden. Hilfreich sei gewesen, dass die Stakeholder auf zuvor unberücksichtigte Hemmnisse hingewiesen und von der Wissenschaft getätigte Annahmen durch Praktiker bestätigt wurden. Darüber hinaus lieferten die Stakeholder aus Sicht eines Befragten interessante fallspezifische Informationen und Hinweise zu Feinheiten der entwickelten Projektsprache, die, so verwendet, gegebenenfalls „Türen schließen kann“. Positiv bewertet wurden auch Hinweise aus der Praxis zu Umsetzungshemmnissen, instrumentellen Schwächen und Defiziten sowie Zielkonflikten und Synergien. Ohne diesen Austausch mit den Stakeholdern wären nach Aussagen eines Wissenschaftlers zwar vermutlich keine grundlegend anderen Ergebnisse entstanden, aber es sei hilfreich, die eigene Arbeit auf die Praxisrelevanz hin prüfen zu lassen: „Als Wissenschaftler schmort man häufig im ,eigenen Saft‘ und verkennt, dass die Schwierigkeiten ,da draußen“ andere sind als man annimmt." Dadurch wurde auch die Einordnung der Umsetzungsprobleme von Maßnahmen in der Praxis möglich.

Die befragten Projektbeteiligten sahen aber auch Grenzen eines solchen transdisziplinären Ansatzes. Die transdisziplinäre Arbeit erfordere einen hohen Grad an Verständnis für die andere Disziplin, häufig fehle aber für weiterführende Kritik ein ausreichender Sachverstand. Zudem wurde kritisch angemerkt, dass die Stakeholder zum Teil zu stark auf lokale Themenstellungen fokussierten, die nicht immer gut zum regionalen bzw. bundesweiten Fokus des Gesamtprojektes passten. Ein Befragter stellte die Notwendigkeit einer distanzierten Sichtweise der Wissenschaft heraus, da es durchaus einzelne Stakeholder gab, die spezifische eigene Interessen verfolgten.

Insgesamt bewerteten sieben der acht Wissenschaftler die Zusammenarbeit mit den Praktikern positiv und sahen gegenüber anderen Forschungsansätzen einen Zusatznutzen für ihre eigene Arbeit. So stellte ein Konsortiumsmitglied heraus, dass durch den transdisziplinären Ansatz die Relevanz der Forschung insbesondere hinsichtlich der Formulierung relevanter Forschungsfragen gesichert werden könne. Den Beitrag dieses Ansatzes sahen andere Wissenschaftler vor allem in der höheren Anwendungsorientierung ihrer Arbeiten und der eigenen Sensibilisierung für die Probleme der Landnutzer. Das trug dazu bei, die Ansichten und Einstellungen zu bestimmten 
Themen besser kennenzulernen sowie sich selber zu disziplinieren und seine Erkenntnisse anderen gegenüber zu vermitteln. Herausgestellt wurden aber auch Probleme in der Umsetzung des Prozesses. Dazu zählten u. a. der hohe Zeit- und Kostenaufwand eines solchen Ansatzes, der Umgang mit Einzelmeinungen und Lobbyismus sowie die Schwierigkeit, Akteure für den Prozess zu gewinnen. Problematisiert wurde darüber hinaus der zum Teil unterschiedliche Kenntnisstand der Stakeholder.

Befragt, wie die Einbindung nicht-wissenschaftlicher Akteure bei einem Folgeprojekt aussehen sollte, antworteten vier Wissenschaftler, dass die Beteiligung genau den Grad haben sollte wie in CC-LandStraD. Drei Befragte plädierten für eine geringere Beteiligung (bei einer fehlenden Angabe). Allerdings empfahl ein Konsortiumsmitglied, wenn das Projekt nochmals von vorn beginnen könnte, weniger Akteure einzubinden und das Thema am Anfang mehr einzuengen. Die Forschungsergebnisse müssten früher vorliegen, um den Diskussionsprozess zu befruchten und auf ein höheres Niveau zu heben. Ein Wissenschaftler wünschte sich für ein mögliches kommendes Projekt eine stärkere Einbindung nicht-organisierter Interessenvertreter.

\subsubsection{Der transdisziplinäre Diskurs: Was hat er gebracht?}

Im Rahmen des transdisziplinären Diskurses in CC-LandStraD wurde eine Vielzahl von regionalen und bundesweiten Interessenvertretern der Landnutzung am Projekt und dessen Fortgang beteiligt. Konkret erfolgte dies, indem:

- einzelne externe Beteiligte über eine längere Zeit mitwirkten,

- weitere Stakeholder punktuell beteiligt waren,

- das Wissen und die Interessen externer Akteure mittels partizipativer Verfahren (Workshops, Fokusgruppen, Interviews) erhoben und einbezogen wurde.

Damit war CC-LandStraD durch einen mittleren Grad an Transdisziplinarität gekennzeichnet (Defila et al. 2006, S. 216). Die Zielsetzung und der Verlauf des Forschungsvorhabens wurden stark durch den Fortgang der Modellierungsaktivitäten geprägt. Zu bestimmten Zeitpunkten - nach einer ersten Auswahl von Maßnahmen und vor Festlegung der endgültigen Strategieausgestaltung - hatten die Stakeholder die Möglichkeit, ihre Interessen und Erwartungen in Bezug auf aktuelle und zukünftige Landnutzungsentwicklungen einzubringen.

Nachfolgend werden zentrale inhaltliche, methodische und prozessuale Aspekte des transdisziplinären Diskurses zusammenfassend dargestellt und reflektiert.

\subsubsection{Inhaltliche Aspekte des transdisziplinären Diskurses}

Die Frage, was nachhaltige Landnutzung unter dem Einfluss des Klimawandels und unter besonderer Berücksichtigung der Verminderung von Treibhausgasen bzw. einer höheren Kohlenstoffspeicherung eigentlich bedeutet, führte das Projektkonsortium 
relativ schnell zu konkreten Klimaschutz- und Klimaanpassungsmaßnahmen (vgl. Kap. 3). Vor Beginn der Forschungsarbeiten war nicht absehbar gewesen, dass dies kein rasch abgeschlossener Baustein, sondern Bestandteil der Projektarbeit über mehrere Jahre sein würde. Die landnutzungsbezogenen Klimaschutz- und Klimaanpassungsmaßnahmen sowie ihre Ausgestaltung in den Strategien (Klimaschutz, Bioenergie, Natur- und Umweltschutz sowie Klimaanpassung) waren auch zentrales Thema der Diskussionen mit den Stakeholdern, da diese ausreichend konkret waren, um an der beruflichen Praxis der eingeladenen Akteure anzuknüpfen. Zur Projekthalbzeit (Anfang 2013) waren 158 solcher Maßnahmen im Gespräch gewesen. Von diesen waren 28 durch das Konsortium, 74 durch die regionalen Akteure und 56 durch die bundesweiten Stakeholder eingebracht worden. 85 erwiesen sich als prinzipiell modellierbar, 73 als nicht modellierbar (Crewett et al. 2013). In einem weiteren Prozess der Verdichtung und (zwangsläufigen) Beschränkung wurde die Zahl zunächst auf 46, abschließend auf 36 modellierbare Maßnahmen verringert.

Bezogen auf die Strategien ging es neben der Priorisierung und den Ausprägungen der zuzuordnenden Maßnahmen vor allem darum, wie praxisnah oder -fern solche Strategien sein sollten - und damit um die generelle Frage der Zielsetzung von Strategien (bzw. Szenarien). Dienen sie vor allem der Verständigung über etwas besonders Wünschenswertes oder nicht Wünschenswertes, oder sollen sie versuchen, tatsächlich zu erwartende Zukünfte so gut wie möglich abzubilden - sind sie also eher normativ oder eher realitätsnah? Im hier dokumentierten Forschungsprozess war dies immer wieder neu zu klären - in einem Projekt mit einem höheren Grad an Transdisziplinarität wäre hingegen ein mit den Stakeholdern gemeinsam entwickeltes Verständnis wünschenswert.

Die Maßnahmen- und Strategiediskussion wurde von der Thematisierung sektoraler und intersektoraler Landnutzungskonflikte begleitet. Diese hatten, wie oben erwähnt, bereits in den leitfadengestützten Interviews eine zentrale Rolle gespielt. Abb. 5.11 stellt die wichtigsten Konflikte zusammenfassend dar (für eine detaillierte Darstellung vgl. Steinhäußer et al. 2015).

Aus der Vielzahl der Landnutzungskonflikte sind aus sektorübergreifender Perspektive zwei besonders hervorzuheben:

- Ein zentrales Konfliktthema stellte aus Sicht der Stakeholder die Sicherung von (landwirtschaftlicher) Fläche dar. Als zentrales Problem wurde aus Sicht der Landund Forstwirtschaft die anhaltende, wenn auch im Bundesdurchschnitt abnehmende Flächenneuinanspruchnahme durch die Expansion von Siedlungen thematisiert. Ebenso wurde die Versiegelung fruchtbarer Böden problematisiert. Nutzungsverzicht, also Flächenstilllegungen für den Naturschutz, galt den Vertretern der Wirtschaftssektoren Land- und Forstwirtschaft nicht als optimale Lösung. Interessenvertreter des Sektors Siedlung und Verkehr stellten dem teilweise eine andere Sicht entgegen, indem sie auf das aus ihrer Sicht veränderte Bewusstsein in Bezug auf die Flächen- 


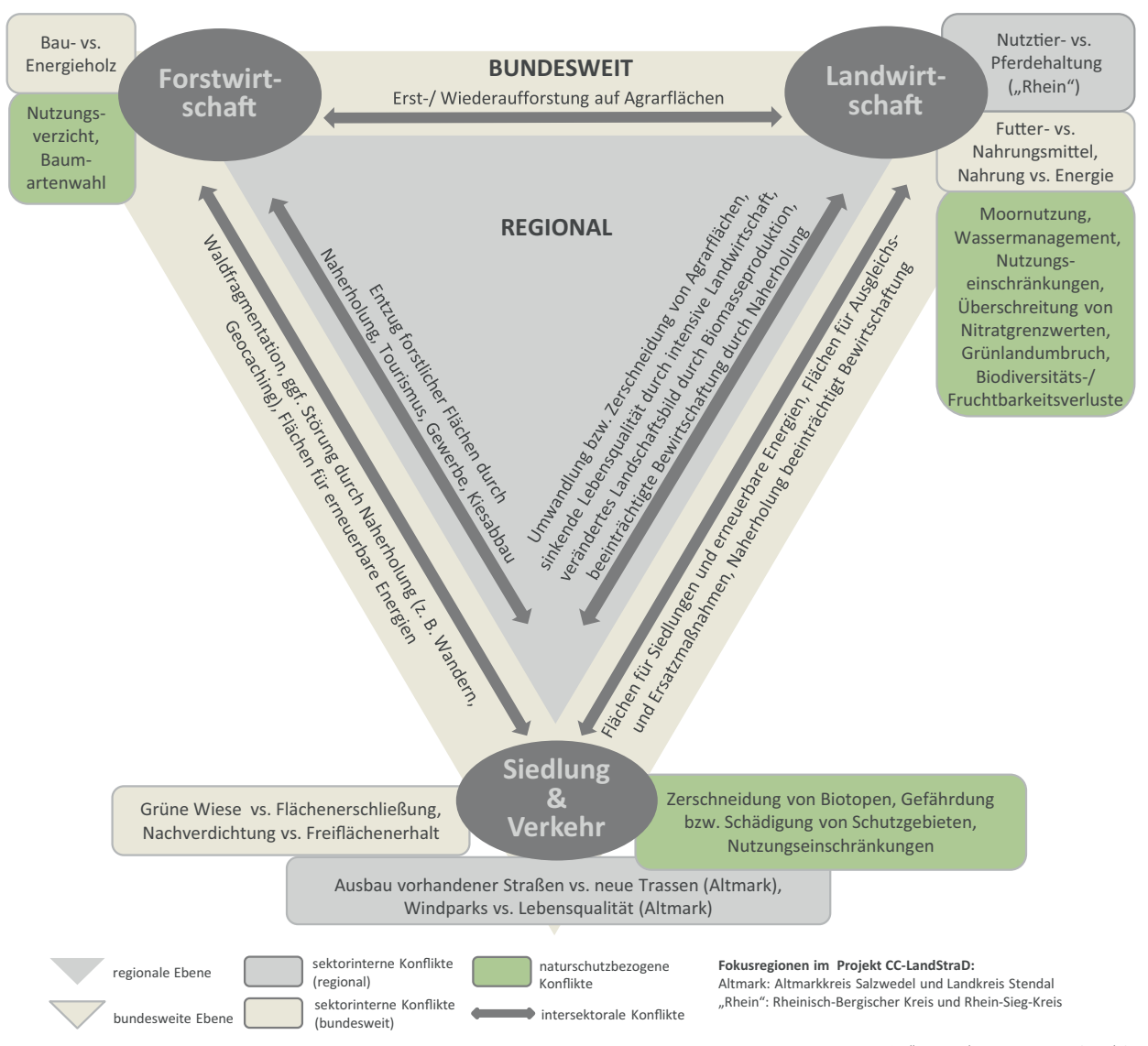

Abb. 5.11 Von den regionalen und bundesweiten Akteuren der Landnutzung im Prozessverlauf thematisierte Landnutzungskonflikte; eigene Zusammenstellung. (Quelle: Steinhäußer et al. 2015, graphische Umsetzung: Nina Röhrig)

neuinanspruchnahme und das proklamierte ,30-Hektar-Ziel“, das für die Planungspraxis eine wichtige (wenn auch nicht erreichbare) Richtschnur sei, hinwiesen.

- Als weitere wichtige Konfliktlinie wurde die in der jüngeren Vergangenheit starke Zunahme der Flächennutzung für die Bioenergieproduktion identifiziert. Als Minimalkonsens zeichnete sich die Formel ab, dass in der Landwirtschaft Bioenergiepflanzen nur als Übergangslösung akzeptiert würden. Mehrfach wurde auf die globale Perspektive (insbesondere die Verlagerung von Landnutzungskonflikten in andere Länder und Probleme der Ernährung der Weltbevölkerung) hingewiesen. Stakeholder der Forstwirtschaft hielten die energetische Holznutzung in Konkurrenz zur stofflichen Nutzung von Holzerträgen ebenfalls für einen der wichtigsten Nutzungskonflikte innerhalb der Forstwirtschaft. Sie betonten, dass durch die „Energie- 
wende" auch die Nachfrage nach Energieholz gestiegen sei, mit der Folge, dass die Konkurrenz in der Holzverwertung zugenommen habe. Beide Sektoren waren sich weitgehend einig darin, dass durch den Bedeutungsgewinn erneuerbarer Energien die ,eigentlichen“ Aufgaben der Forst- und Landwirtschaft nicht „,vergessen“ werden sollten.

Die inhaltliche Begrenzung des Projektes auf sektorale Beiträge zum Klimaschutz sowie, in der Forstwirtschaft und im Siedlungswesen, auf Klimaanpassungsmaßnahmen wurde von den Akteuren zwar einerseits begrüßt, andererseits mahnten sie im Prozessverlauf immer wieder eine umfassendere gesellschaftliche Perspektive an: Nachhaltige Landnutzung könne nicht nur isoliert klimatische Veränderungen (also einen Aspekt ökologischer Nachhaltigkeit) berücksichtigen. Problematisiert wurden zum Beispiel die Grenzen solcher Nachhaltigkeit, wenn die Einkommen von Landwirtschaft und Forstwirtschaft nicht mehr gesichert sind, zentrale Aufgaben der Sektoren in den Hintergrund rücken und/oder die Lebensqualität sinkt. Die Interessenvertreter der Landnutzung betonten, dass Klimawandel für Landnutzer nur ein Problem unter anderen (wenn überhaupt) sei. In ihrer Arbeit spielen beispielsweise Fragen der Gemeinsamen Agrarpolitik der EU, die Novellierung der Düngeverordnung, Wertschöpfungsketten, Holzpreise oder die Sicherung der Daseinsvorsorge und die Leerstandsproblematik eine mindestens ebenso wichtige Rolle.

Hervorzuheben ist in diesem Zusammenhang, dass der formulierte Projektanspruch der Nachhaltigkeit von den Akteuren der Landnutzung so ernst genommen wurde, dass sie ganzheitliche bzw. umfassendere Modelle und ein höheres Maß an sektorübergreifender Integration einforderten, als es CC-LandStraD - das in seinen Modellierungen oft Neuland betreten und sich gleichzeitig beschränken musste - in jedem Falle einlösen konnte. Auch die (fehlende) Nachhaltigkeit von Wissenschaft selbst wurde thematisiert, z. B. bezogen auf die Zeitdauer von Forschungsprojekten oder die aus Sicht der Praxis nicht immer zufriedenstellende Bekanntheit abgeschlossener Projekte und ihrer Ergebnisse.

\subsubsection{Methodische Aspekte des transdisziplinären Diskurses}

Im Laufe der transdisziplinären Projektarbeit gab es immer wieder „Übersetzungsbedarf" zwischen der Vorgehensweise der Wissenschaftler und den Anforderungen der Stakeholder. Aus methodisch-methodologischer Perspektive wurden Inhalte und Zweck der verwendeten Modelle regelmäßig kritisch hinterfragt. Das ging zum Teil mit widersprüchlichen Anforderungen einher: Einerseits wiesen die Stakeholder darauf hin, dass die Modellierung komplexe Wechselwirkungen in den Bereichen Ökonomie (z. B. die Relation von Agrar- und Energiepreisentwicklungen) und Landnutzungsentscheidungen abbilden solle, um Relevanz zu besitzen - ein Anspruch, dem sich die verschiedenen Modelle im Rahmen ihrer jeweiligen Modellgrenzen auch stellten. Andererseits forderten die Stakeholder Vereinfachung und klare bzw. keine widersprüchlichen Befunde ein. Zugleich wurde in den Workshops deutlich, dass eine Ergeb- 
nisinterpretation und -einordnung für die Stakeholder aufgrund der Modellkomplexität zum Teil schwierig waren. Sich teilweise widersprechende Modellergebnisse und die (notwendige) Vernachlässigung weiterer Wechselwirkungen und gesellschaftlicher Rahmenbedingungen - die Modelle nur zum Teil oder gar nicht berücksichtigen können - führten teilweise zu Skepsis im Umgang mit Modellergebnissen. Dieser Befund erklärt sich aus den unterschiedlichen Systemlogiken von Wissenschaft einerseits und Interessenverbänden andererseits. Verbände und Behörden verfolgen andere Interessen und Lösungsansätze, die sich mit denen der Wissenschaft nur bedingt überschneiden. Dieser Konflikt war im Prozess nicht gänzlich lösbar, da es sich eben nicht nur um ein Problem von Fachjargon und Modellsprache handelte.

Von einzelnen Stakeholdern wurde die Befürchtung geäußert, dass durch ,plakative“ Szenarien bei der Bevölkerung oder der Politik ein falscher Eindruck über die Landnutzung entstehen könnte. Ferner gab es auch den Appell an die Wissenschaftler, sich nicht in $\mathrm{zu}$ vielen unüberschaubaren Modellen $\mathrm{zu}$ verlieren, sondern $\mathrm{zu}$ versuchen, „einfache“ Botschaften zu produzieren. Das Forschungskonsortium musste lernen, in den Workshops stets von neuem wieder auf die Rolle, Möglichkeiten und Grenzen von Modellen hinzuweisen und zu kommunizieren, dass es nicht um eine Abbildung der Realität auf kleinräumiger Ebene geht, sondern um Zuspitzungen und Vereinfachungen.

Praxisakteure erwarten von einem solchen Projekt in einem überschaubaren Zeitraum innovative, praktische Lösungen und somit wissenschaftlich fundierte Handlungsempfehlungen, die noch dazu regional angepasst sein sollten. Die Modelle und Modellierungsergebnisse können - und sollen - dies nur in sehr begrenztem Maße leisten. Auch über die Ziele von CC-LandStraD - jenseits besserer Daten und Wissen über Wechselwirkungen - gab es kontinuierlichen Verständigungsbedarf: Nicht jedem Akteur war klar (oder vermittelbar), dass das Konsortium am Projektende keine Forderungen an die Politik oder ausgearbeitete Handlungsvorschläge unterbreiten werde. Gleichzeitig warnten einige Beteiligte davor, dass allgemeine Politikempfehlungen auf Basis einer Modellierung zu unrealistischen Annahmen, etwa in den zuständigen Ministerien, führen könnten, die daraufhin möglicherweise Entscheidungen treffen, die wiederum an die Kommunen weitergegeben werden. Es sollten keine deutschlandweiten, sondern situativ auf die jeweilige Region bezogene Aussagen getroffen werden.

$\mathrm{Zu}$ einem erfolgreichen transdisziplinären Projekt gehört somit auch, die Erwartungen aller Beteiligten sowie die Ziele der Beteiligungsprozesse nicht nur im Vorhinein genau zu klären, sondern diese Frage im Prozess immer wieder aufzurufen. Gleichzeitig liegt es in der Logik von Beteiligungsprozessen, dass sich die Stakeholder sowohl innerhalb als auch zwischen den verschiedenen Landnutzungssektoren in ihren Positionen und Meinungen unterschieden und teilweise explizit als Lobbyisten auftraten. Insbesondere der Umgang mit Lobbypositionen bleibt ein transdisziplinären Projekten inhärentes Problem, das auch in künftigen Projekten nicht durch verbesserte Kommunikation allein zu lösen sein wird. Der Abwägungsprozess, welche Meinungen mit welchem Gewicht für die weiteren Projektschritte berücksichtigt werden sollten, oblag den Fachwissenschaftlern. Der Beteiligungsprozess wurde dadurch erschwert, dass die Stakeholder-Ver- 
bände auf Bundesebene (anders als im regionalen Beteiligungsprozess) nicht in jedem Falle eine Kontinuität der teilnehmenden Personen gewährleisten konnten, sodass neue Teilnehmer mit den Modellen und den Ergebnissen von Vorläuferworkshops nicht ausreichend vertraut waren.

\subsubsection{Prozessuale Aspekte}

Auf Unstetigkeiten der Stakeholder-Beteiligung an den Projektaktivitäten ist im Zusammenhang mit den Tab. 5.8 und 5.9 bereits hingewiesen worden. Die Teilnahme an Workshops war mit einem hohen persönlichen Zeitaufwand verbunden, auch wenn vonseiten des Projektes versucht wurde, diesen für die Stakeholder möglichst gering zu halten, zum Beispiel durch halbtägige Veranstaltungen im regionalen Beteiligungsprozess oder durch die Wahl des Ortes. Bei Bedarf wurden Mittel für Aufwandsentschädigungen und/oder Reisekosten bereitgestellt. Termine für die Fokusgruppen und Workshops wurden möglichst frühzeitig angekündigt und mit den Stakeholdern abgestimmt. Es erwies sich auch als positiv, den Workshopeinladungen eine inhaltliche Ergebnisorientierung, gut aufgemachte Zwischenergebnisse oder eine Tischvorlage beizufügen. Dennoch führte dies insbesondere im bundesweiten Prozess nicht immer zu optimaler Beteiligung.

Bedingt durch die einzelnen Phasen der Modellierung, die sektoral mit unterschiedlichen Zeithorizonten erfolgte, gab es für die Hauptaktivitäten mit den Stakeholdern die interaktiven Workshops - keinen festen Rhythmus. Da, wie oben bereits erwähnt, auf Bundesebene die personelle Situation bei den Verbänden, Ämtern und Ministerien nicht konstant blieb, konnte nicht in jedem Falle an eine Vorgeschichte angeknüpft, sondern mussten Kontakte wieder neu aufgebaut werden. Stakeholder-Arbeit ist somit - ebenso wie die Analyse der Akteursstruktur (Stakeholder-Mapping der Institutionen und Personen in den betreffenden Organisationen) - ein kontinuierlicher und projektbegleitender Prozess. In kritischer Reflexion ist zu sagen, dass über den halbjährlichen Newsletter-Versand hinaus in stärkerem Maße direkte Kontakte hätten gepflegt werden sollen, um das Projekt in Erinnerung zu halten und die Türen für eine künftige Beteiligung noch stärker offenzuhalten. Auch der Wechsel von Personen in Verbänden und Behörden sollte antizipiert werden. Hier könnte z. B. ansprechendes Informationsmaterial über das Projekt die potenzielle Beteiligungsbereitschaft verbessern.

Die prozessuale Offenheit des transdisziplinären Diskurses hat sich hingegen bewährt - zwar lässt sich vor einem solchen Projekt planen, welche Methoden in welcher Reihenfolge und auf welcher räumlichen Ebene angewendet werden sollten, doch ist ein solch komplexes Modellierungsvorhaben wie CC-LandStraD immer von Unstetigkeiten und Unplanbarkeiten begleitet. Wie oben erwähnt, verlängerten beispielsweise die mehrfachen Workshop-Diskussionen die Phase der konkreten Ausgestaltung der Maßnahmen und Strategien in den Sektormodellen, aber so entstand auch eine bessere Validierung dieser zentralen Projektbausteine durch die Stakeholder. 


\subsubsection{Schlussfolgerungen}

Dass sich Projektantrag und -verlauf zwangsläufig unterscheiden, ist der Natur wissenschaftlichen Erkenntnisgewinns geschuldet. Durch den transdisziplinären Diskurs ist CC-LandStraD nochmals ein anderes Forschungsprojekt geworden, als zur Antragsphase absehbar gewesen war. Fünf Workshops für bundesweite Interessenvertreter, zwölf Fokusgruppen und Workshops auf regionaler Ebene sowie ein gemeinsamer Workshop führten zu zahlreichen inhaltlichen, methodischen und prozessualen Detailänderungen im Projektverlauf. Zwar waren für diesen transdisziplinären Diskurs ausreichend Ressourcen eingeplant gewesen, doch war der kontinuierliche Zeitaufwand für die Fachwissenschaftler des Konsortiums unterschätzt worden. Transdisziplinarität verändert nicht nur die Zeitplanung für die eigentliche (Modellierungs-)Arbeit, sondern bringt einen aufwendigen Übersetzungsbedarf für alle Beteiligten mit sich und stellt hohe Ansprüche an die Kommunikation mit nicht-wissenschaftlichen Akteuren, z. B. hinsichtlich einer größeren Modelltransparenz.

Nach fünf Jahren CC-LandStraD kann festgehalten werden, dass sich Akteure der Landnutzung mit großem Organisations- und Kommunikationsaufwand für solche Projekte und ihre Begleitung gewinnen lassen. Wunsch und Realität der tatsächlichen, aktiven Beteiligung klaffen aber zum Teil aufseiten der Akteure und zum Teil auch der Wissenschaft angesichts ihrer eigentlichen Aufgaben auseinander. Das spricht dafür, Stakeholder im Sinne einer „starken“ Transdisziplinarität bereits in der Antragsphase an der Problemdefinition und im Projektverlauf als gleichberechtigte (das heißt auch: bezahlte) Partner zu beteiligen (Jahn und Keil 2006, S. 327) - mit der Folge, dass ein solcher Ansatz die Rolle der Wissenschaftler verändert und die Ergebnisse der Forschungsarbeit weniger vorhersehbar macht, was aus Perspektive von Drittmittelgebern nicht immer wünschenswert ist. Auch wird man sich dann in der Zahl der Beteiligten einschränken und deren Auswahl noch stärker inhaltlich begründen müssen.

Transdisziplinarität bedeutet immer ein Sich-Einlassen auf unterschiedliche Wissensbestände, Weltzugänge und Problemverständnisse und damit verbunden einen hohen Übersetzungsaufwand. Wenn dies bereits in der Antragsphase im wechselseitigen Einverständnis geklärt ist und im Projektverlauf kontinuierlich reflektiert wird, steht einem „Mehr“ an Einbindung von wissenschaftsexternen Partnern nichts entgegen. Für die Projektstruktur und die Ziele von CC-LandStraD war der mittlere Grad an Transdisziplinarität angemessen.

\section{Literatur}

Abildtrup J, Garcia S, Stenger A (2013) The effect of forest land use on the cost of drinking water supply: a spatial econometric analysis. Ecol Econ 92:126-136

Achterkamp MC, Vos JFJ (2008) Investigating the use of the stakeholder notion in project management literature, a meta-analysis. Int J Project Manage 26(3):749-757 
adelphi/PRC/EURAC (2015) Vulnerabilität Deutschlands gegenüber dem Klimawandel. Umweltbundesamt. Clim Change 24, Dessau-Roßlau

Agentur für Erneuerbare Energien (AEE) (2015) Akzeptanz für Erneuerbare Energien weiterhin hoch. Ergebnisse der TNS Emnid-Umfrage 8/2015. Renews kompakt 23:1-3

Aidt TS (1998) Political internalization of economic externalities and environmental policy. J Public Econ 69:1-16

Ammer C, Vor T, Knoke T, Wagner S (2010) Der Wald-Wild-Konflikt. Analyse und Lösungsansätze vor dem Hintergrund rechtlicher, ökologischer und ökonomischer Zusammenhänge. Gutachten. BfN, DFWR, ANW. Universitätsverlag Göttingen, Berlin, Bonn

Arbeitsgemeinschaft der für Städtebau, Bau- und Wohnungswesen zuständigen Minister und Senatoren (ARGEBAU), Bund/Länder Arbeitsgemeinschaft Wasser (LAWA), Ministerkonferenz für Raumordnung (MKRO) (2010) Handlungsanleitung für den Einsatz rechtlicher und technischer Instrumente zum Hochwasserschutz in der Raumordnung, in der Bauleitplanung und bei der Zulassung von Einzelbauvorhaben. Beschlussfassung Fachkommission Städtebau vom 22. September 2010

Arlt G, Pfeil F (1996) Flächennutzungskonkurrenzen - Typisierung von Nutzungskonflikten. In: Brake K, Richter U (Hrsg) Methoden zur Analyse und Bewertung von Flächennutzungsund Standortmustern. Nachhaltige Entwicklung von Großstadtregionen. Bibliotheks- und Informationssystem der Universität, Oldenburg, S 27-44

Arnstein S (1969) A ladder of citizen participation. J Am Plann Assoc 35(4):216-224

Bateman IJ, Mace GM, Fezzi C, Atkinson G, Turner K (2011) Economic analysis for ecosystem service assessments. Environ Resource Econ 48(2):177-218

Becker E, Jahn T (2006) Soziale Ökologie - Konstitution und Kontext. In: Becker E, Jahn T (Hrsg) Soziale Ökologie. Grundzüge einer Wissenschaft von den gesellschaftlichen Naturverhältnissen. Campus, Frankfurt a. M., S 29-89

Begg C, Luther J, Kuhlicke C, Steinführer A (2011) Participation in Central European flood risk management: social capacity building in practice. CapHaz-Net WP9 Report. Helmholtz Centre for Environmental Research - UFZ, Leipzig

Berlin-Institut für Bevölkerung und Entwicklung (2011) Die Zukunft der Dörfer. Zwischen Stabilität und demografischem Niedergang. In: Kröhnert S, Kuhn E, Karsch M, Klingholz R, Bennert W (Hrsg) Autoren. Selbstverlag, Köln

Biernacki P, Waldorf D (1981) Snowball sampling: problems and techniques of chain referral sampling. Sociol Method Res 10(2):141-163

Birner R, lsermeyer F, Lang C, Treffenfeldt W, Zinke H (Bioökonomierat) (2014) Landwirtschaft in Deutschland - ihre Rolle für die Wettbewerbsfähigkeit der Bioökonomie. BÖRMEMO 01, Berlin

Bracher T, Gies J, Schlünder I, Warnecke T (2014) Finanzierung des ÖPNV durch Beiträge. Ist das Beitragsmodell eine Handlungsoption zur Finanzierung eines attraktiven ÖPNV-Betriebs? DIfU-Paper, Berlin

Bräuer I, Marggraf R (2004) Valuation of ecosystem services provided by biodiversity conservation: an integrated hydrological and economic model to value the enhanced nitrogen retention in renaturated streams. FEEM Working Paper No. 54.04, Milan, Italien

Brugha R, Varvasovszky Z (2000) Stakeholder analysis: a review. Health Policy Plann 15(3):239_ 246

Bund/Länder-Arbeitsgemeinschaft Wasser (LAWA) (Hrsg) (2014) Nationales Hochwasserschutzprogramm. Beschlossen auf der Umweltministerkonferenz am 24. Oktober 2014 in Heidelberg. Heidelberg. https://www.bmub.bund.de/fileadmin/Daten_BMU/Download_PDF/ Binnengewaesser/hochwasserschutzprogramm_bericht_bf.pdf. Zugegriffen: 12. März 2018 
Bundesamt für Naturschutz (BfN) (2012) Energieholzanbau auf landwirtschaftlichen Flächen. Auswirkungen von Kurzumtriebsplantagen auf Naturhaushalt, Landschaftsbild und biologische Vielfalt. Anbauanforderungen und Empfehlungen des BfN. Bonn, Bad Godesberg. https://www. bfn.de/fileadmin/MDB/documents/themen/erneuerbareenergien/bfn_energieholzanbau_landwirtschaftliche_flaechen.pdf. Zugegriffen: 12. März 2018

Bundesministerium für Ernährung und Landwirtschaft (BMEL) (2013) Wirtschaftliche Lage der forstwirtschaftlichen Betriebe. Buchführungsergebnisse der Testbetriebe des Forstwirtschaftsjahres 2012. Bonn. https://www.bmelv-statistik.de/fileadmin/user_upload/monatsberichte/BFB0113000-2012.pdf. Zugegriffen: 12. März 2018

Bundesministerium für Ernährung und Landwirtschaft (BMEL) (2014) Die wirtschaftliche Lage der landwirtschaftlichen Betriebe. Buchführungsergebnisse der Testbetriebe. Wirtschaftsjahr 2013/2014. Bonn

Bundesministerium für Ernährung und Landwirtschaft (BMEL) (2015) Bundeswaldinventur 3. www.bundeswaldinventur.de. Zugegriffen: 19. März 2019

Bundesministerium für Umwelt, Naturschutz und nukleare Sicherheit (BMU) (2007) Nationale Strategie zur biologischen Vielfalt. Berlin, S 178

Bundesministerium für Verkehr, Bau und Stadtentwicklung (BMVBS) (Hrsg) (2012) Regionalplanerische Instrumente zur Reduzierung der Flächeninanspruchnahme. BMVBS-OnlinePublikation 20/2012. Berlin

Bundesministerium für Verkehr und digitale Infrastruktur (BMVI) (Hrsg) (2014) Grundkonzeption für den Bundesverkehrswegeplan 2015. Bedarfsgerecht - transparent - herausfordernd. Bonn. https://www.bmvi.de/SharedDocs/DE/Anlage/VerkehrUndMobilitaet/BVWP/bvwp-2015grundkonzeption-langfassung.html. Zugegriffen: 12. März 2018

Bundesregierung (2002) Perspektiven für Deutschland Unsere Strategie für eine nachhaltige Entwicklung. Bundeskanzleramt, Berlin, S 343

Bundesregierung (2011) Aktionsplan Anpassung der Deutschen Anpassungsstrategie an den Klimawandel. Bundesregierung. https://www.bmu.de/fileadmin/bmu-import/files/pdfs/allgemein/application/pdf/aktionsplan_anpassung_klimawandel_bf.pdf. Zugegriffen: 15. Nov. 2019

Bundesverband der Energie- und Wasserwirtschaft (BDEW) (2015) Vergleich Europäischer Wasser- und Abwasserpreise. BDEW, Berlin

Bunzel A, Frölich v. Bodelschwingh F, Strauss WC (2014) Grundlagenforschung zur Baugebietstypologie der Baunutzungsverordnung. Endbericht. Deutsches Institut für Urbanistik. Berlin BWaldG (Bundeswaldgesetz) Gesetz zur Erhaltung des Waldes und zur Förderung der Forstwirtschaft vom 02.05.1975 (BWaldG BGB1 I, S 1037), zuletzt geändert 31.07.2010

Crewett W, Hellmich M, Siebert R, Steinführer A (2013) Nachhaltiges Landmanagement aus Sicht von Akteuren der Landnutzung. Vortrag auf der Zwischenkonferenz in Braunschweig, 25./26. Februar 2013

Czybulka D, Hampicke U, Litterski B (Hrsg) (2012) Produktionsintegrierte Kompensation. Rechtliche Möglichkeiten, Akzeptanz, Effizienz und naturschutzgerechte Nutzung. Schmidt, Berlin

De Marchi B (1991) The Seveso directive: an Italian pilot study in enabling communication. Risk Anal 11(2):207-215

Defila R, Di Giulio A, Scheuermann M (2006) Forschungsverbundmanagement Handbuch für die Gestaltung inter- und transdisziplinärer Projekte. vdf Hochschulverlag, Zürich

Destatis (2014) Bevölkerung und Erwerbstätigkeit - Vorläufige Ergebnisse der Bevölkerungsfortschreibung auf Grundlage des Zensus 2011, Artikelnummer: 5124103119005. Statistisches Bundesamt, Wiesbaden

Deutsche Vereinigung für Wasserwirtschaft, Abwasser und Abfall e. V. (DWA) (2015) 27. Leistungsvergleich kommunaler Kläranalagen 2014. Koblenz 
Domínguez-Torreiro M, Soliño M (2011) Provided and perceived status quo in choice-experiments: implications for valuing the outputs of multifunctional rural areas. Ecol Econ 70(12):2523-2531

Edenhofer O, Flachsland C, Kalkuhl M, Knopf B, Pahle M (2019) Optionen für eine $\mathrm{CO}_{2}$-Preisreform. Arbeitspapier No. 04/2019. Sachverständigenrat zur Begutachtung der Gesamtwirtschaftlichen Entwicklung, Wiesbaden

Einig K (2011) Die Flächenwende kommt nicht von allein. LandInForm 2(2011):14-15

Elsasser P, Weller P (2013) Aktuelle und potentielle Erholungsleistung der Wälder in Deutschland: Monetärer Nutzen der Erholung im Wald aus Sicht der Bevölkerung. Allgemeine Forst- und Jagdzeitung 184(3/4):83-95

Elsasser P, Englert H, Hamilton J (2010) Landscape benefits of a forest conversion programme in North East Germany: results of a choice-experiment. Ann For Res 53(1):37-50

Elsasser P, Bösch M, Leefken G, Möhring B, Pistorius T, Rock J, Rüter S, Schröppel B (2015) Klimaschutz als Ökosystemleistung des Waldes in Deutschland: Wie tragen deutsche Wälder zum Schutz der Atmosphäre bei? In: Hartje V, Wüstemann H, Bonn A (Hrsg) Naturkapital und Klimapolitik: Synergien und Konflikte. Naturkapital Deutschland - TEEB DE. TUB \& UFZ, Berlin \& Leipzig, S 148-170

European Energy Exchange (2016) Emission spot primary market auction report. https://www.eex. com/en/market-data/environmental-markets/auction-market/european-emission-allowancesauction/european-emission-allowances-auction-download. Zugegriffen: 18. Apr. 2019

Finke P (2014) Citizen Science. Das unterschätzte Wissen der Laien. Oekom, München

Förster R, Pohl C, Scheringer M, Valsangiacomo A (2001) Partizipation in der transdisziplinären Forschung - Eine Positionierung und die Ankündigung des nächsten SAGUFNET-Workshops. GAIA 10(2):146-149

Fraunhofer-Institut für System- und Innovationsforschung (ISI), Gesellschaft für Wirtschaftliche Strukturforschung mbH (GWS), Institut für ZukunftsEnergieSysteme (IZES) und Deutsches Institut für Wirtschaftsforschung (DIW) (2011) Methodische Ansätze zur Analyse der Kostenund Nutzenwirkungen des Ausbaus Erneuerbarer Energien im Wärmebereich, Studie im Auftrag des Bundesministeriums für Umwelt, Naturschutz und Reaktorsicherheit, Nov. 2011

Freeman RE (1984) Strategic sanagement: a stakeholder approach. Basic Books, New York

Freeman RE (2004) The stakeholder approach revisited. Zeitschrift für Wirtschafts- und Unternehmensethik 5(3):228-241

Freibauer A, Röder N, Tiemeyer B (2012) Ansätze für die Definition für Gebietskulissen für den GLÖZ-Standard 7: „Schutz von Feuchtgebieten und kohlenstoffreichen Böden einschließlich eines Erstumbruchverbots“. Arbeitsberichte aus dem vTI-Institut für Agrarrelevante Klimaforschung 2012/2010. Braunschweig

Frelih-Larsen A, MacLeod M, Osterburg B, Eory AV, Dooley E, Kätsch S, Naumann S, Rees B, Tarsitano D, Topp K, Wolff A, Metayer N, Molnar A, Povellato A, Bochu JL, Lasorella MV, Longhitano D (2014) Mainstreaming climate change into rural development policy post 2013. Final report. Ecologic Institute, Berlin

Fürst D, Scholles F (2008a) Das System der räumlichen Planung in Deutschland. In: Fürst D, Scholles F (Hrsg) Handbuch Theorien und Methoden der Raum- und Umweltplanung, 3. Aufl. Dorothea Rohn, Dortmund, S 70-99

Fürst D, Scholles F (Hrsg) (2008b) Handbuch Theorien und Methoden der Raum- und Umweltplanung, 3. Aufl. Dorothea Rohn, Dortmund

Glicken J (2000) Getting stakeholder participation 'right': a discussion of participatory processes and possible pitfalls. Environ Sci Policy 3(6):305-310

Gömann H, Bender A, Bolte A, Dirksmeyer W, Englert H, Feil JH, Frühauf C, Hauschild M, Krengel S, Lilienthal H, Löpmeier FJ, Müller J, Mußhoff O, Natkhin M, Offermann F, Seidel 
P, Schmidt M, Seintsch B, Steidl J, Strohm K, Zimmer Y (2015) Agrarrelevante Extremwetterlagen und Möglichkeiten von Risikomanagementsystemen. Studie im Auftrag des Bundesministeriums für Ernährung und Landwirtschaft (BMEL). Braunschweig: Johann Heinrich von Thünen-Institut (Thünen Report 30)

Gren I-M (1995) The value of investing in wetlands for nitrogen abatement. Eur Rev Agric Econ 22:157-172

Grünebaum T et al (2006) Hinweise zu Produktionskosten der kommunalen Abwasserbehandlung. KA - Abwasser, Abfall 2006 53(11)

Haber W, Bückmann W (2013) Nachhaltiges Landmanagement, differenzierte Landnutzung und Klimaschutz. Universitätsverlag der TU Berlin, Berlin

Hanley N, Barbier EB (2009) Pricing nature - cost-benefit analysis and environmental policy. Elgar, Cheltenham

Hansjürgens B (2015) Zur Neuen Ökonomie der Natur: Kritik und Gegenkritik. Wirtschaftsdienst 95:284-291

Hartje V, Meyerhoff J, Dehnhardt A (2003) Monetäre Bewertung einer nachhaltigen Entwicklung der Stromlandschaft Elbe. Forschungsvorhaben gefördert durch das Bundesministerium für Bildung und Forschung (BMBF) im Rahmen des Programms Elbe-Ökologie Förderkennzeichen: 0339594/1 Endbericht Dezember 2003, Berlin, S 81

Hartje V, Wüstemann H, Bonn A (Hrsg) (2015) Naturkapital Deutschland - TEEB DE Naturkapital und Klimapolitik - Synergien und Konflikte. Technische Universität Berlin, HelmholtzZentrum für Umweltforschung - UFZ, Berlin, Leipzig

Hellmich M, Steinführer A (2012) Klimawandel im Spannungsfeld unterschiedlicher Landnutzungsformen. Wahrnehmungen und Bewertungen von Akteuren der Landnutzung. Unter Mitarbeit von Jürges N. Braunschweig: Johann Heinrich von Thünen-Institut (CC-LandStraDArbeitsbericht 1)

Hirschfeld J, Sagebiel J (2014) Äpfel, Birnen und Biodiversität. Ökologisches Wirtschaften 29:25-26

Isermeyer F (2012) Erst die Mittel, dann das Ziel? Wie sich die EU-Agrarpolitik in eine Sackgasse manövriert und wie sie dort wieder herauskommen kann. In: Lange J (Hrsg) Die Begrün(d)ung der Gemeinsamen Agrarpolitik? Die kommende Reform der GAP. Loccumer Protokolle 05/12. Rehburg-Loccum, S 19-62

Isermeyer F (2014) Künftige Anforderungen an die Landwirtschaft - Schlussfolgerungen für die Agrarpolitik. Thünen Working Paper 30. Braunschweig

Isermeyer F, Forstner B, Nieberg H, Offermann F, Osterburg B, Schmidt T, Röder N, Weingarten P (Thünen-Institut) (2014) Gesetzentwurf zur Durchführung der Direktzahlungen an Inhaber landwirtschaftlicher Betriebe im Rahmen von Stützungsregelungen der Gemeinsamen Agrarpolitik. Stellungnahme im Rahmen einer öffentlichen Anhörung des Ausschusses für Ernährung und Landwirtschaft des Deutschen Bundestages am 7. April. 2014. Ausschuss für Ernährung und Landwirtschaft. Ausschussdrucksache 18(10)052-E. https://literatur.ti.bund.de/digbib_ extern/dn053441.pdf. Zugegriffen: 12. März 2018

Jahn T, Keil F (2006) Transdisziplinärer Forschungsprozess. In: Becker E, Jahn T (Hrsg) Soziale Ökologie. Grundzüge einer Wissenschaft von den gesellschaftlichen Naturverhältnissen. Campus, Frankfurt a. M., S 319-329

Jensen R (2015) Vertragsmodelle auf Moorstandorten. In: von Hartje V, Wüstemann H, Bonn A (Hrsg) Naturkapital Deutschland - TEEB DE Naturkapital und Klimapolitik - Synergien und Konflikte. Technische Universität Berlin, Helmholtz-Zentrum für Umweltforschung - UFZ, Berlin, Leipzig, S 197-198

Jessel B, Jacobs J (2005) Land use scenario development and stakeholder involvement as tools for watershed management within the Havel River Basin. Limnologica 35(3):220-233

Joosten H, Schröder C (2014) VIP - Vorpommern Initiative Paludikultur. Endbericht, Greifswald 
Kabat P, van Vierssen W, Veraart J, Vellinga P, Aerts J (2005) Climate proofing the Netherlands. Nature 438(17):283-284

Knauf M, Frühwald A (2013) Beitrag des NRW Clusters ForstHolz zum Klimaschutz. Studie von Knauf Consulting und Prof. Dr. Arno Frühwald (Zentrum Holzwirtschaft der Universität Hamburg) in Kooperation mit Prof. Dr. Michael Köhl (Zentrum Holzwirtschaft der Universität Hamburg) im Auftrag des Ministeriums für Klimaschutz, Umwelt, Landwirtschaft, Natur- und Verbraucherschutz des Landes Nordrhein-Westfalen und des Landesbetriebs Wald und Holz Nordrhein-Westfalen. Düsseldorf, Münster

Kommission Landwirtschaft beim Umweltbundesamt (KLU) (Hrsg) (2014) Umweltverträgliche Biogaserzeugung. Empfehlungen der Kommission Landwirtschaft beim Umweltbundesamt (KLU) zur Reform des EEG. Position. Dessau-Roßlau. https://www.umweltbundesamt.de/ publikationen/umweltvertraegliche-biogaserzeugung-0. Zugegriffen: 12. März 2018

Krueger R, Casey MA (2000) Focus groups. A practical guide for applied research, 3. Aufl. Sage, Thousand Oaks

Kuhlicke C, Steinführer A, Meyer V (2013) Jenseits der Leitdifferenz von „Beton contra Natur": Neue Paradoxien und Ungleichheiten im Hochwasserrisikomanagement. Hydrol Wasserbewirtsch 57(2):70-74

Küpper P, Steinführer A, Ortwein S, Kirchesch M (2013) Regionale Schrumpfung gestalten. Handlungsspielräume zur langfristigen Teilhabe schaffen und nutzen. Johann Heinrich von ThünenInstitut und Bundesanstalt für Landwirtschaft und Ernährung. Braunschweig, Bonn

Landesamt für Umwelt, Wasserwirtschaft und Gewerbeaufsicht Rheinland-Pfalz (LUWG) (Hrsg) (2005) Ökoeffizienzprüfung in der Eingriffsregelung in Rheinland-Pfalz. Bearbeitung: Planungsgruppe Ökologie + Umwelt GmbH, Oppenheim

Löschel A, Sturm B, Vogt C (2013) The demand for climate protection - empirical evidence from Germany. Econ Lett 118(3):415-418

Marschall I et al (2012) Vilmer Visionen 2012. Perspektiven und Herausforderungen für die Landschaftsplanung als Beitrag zu einer nachhaltigen Landschaftsentwicklung. Ergebnis des Expertenworkshops vom 14. bis 16.05.2012 im Bundesamt für Naturschutz - Internationale Naturschutzakademie Insel Vilm. https://www.bfn.de/fileadmin/BfN/planung/landschaftsplanung/Dokumente/vilmer_visionen_2012_barrierefrei.pdf. Zugegriffen: 12. März 2018

Marsh D, Mkwara L, Scarpa R (2011) Do respondents' perceptions of the status quo matter in non-market valuation with Choice-Experiments? An application to New Zealand freshwater streams. Sustainability 3(9):1593-1615

Mayring P (2010) Qualitative Inhaltsanalyse. Juventa, Weinheim

Meyerhoff J, Angeli D, Hartje V (2012) Valuing the benefits of implementing a national strategy on biological diversity - the case of Germany. Environ Sci Policy 23:109-119

Meyerhoff J et al (2014) Der gute ökologische Zustand: Eine Kosten-Nutzen-Analyse für die Untere Havel. Präsentation auf dem NITROLIMIT Projektseminar am 12.05.2014, Berlin

Millennium Ecosystem Assessment (MA) (2005) Ecosystems and human well-being: Synthesis. Island, Washington

Ministerkonferenz für Raumordnung (MKRO) (2013) Raumordnung und Klimawandel. Handlungskonzept der Raumordnung zu Vermeidungs-, Minderungs- und Anpassungsstrategien in Hinblick auf die räumlichen Konsequenzen des Klimawandels vom 23.01.2013. Berlin. https://www. klimamoro.de/fileadmin/Dateien/Transfer_KlimaMORO/Beratungsmodul/Leitf\%C3\%A4den/ Leitf\%C3\% A4den\%20mit\%20Ma\%C3\%9Fnahmenvorsch1\%C3\% A4gen\%20in\% 20 relevanten\%20Handlungsfeldern/MKRO-Handlungskonzept\%20der\%20Raumordnung\%20.pdf. Zugegriffen: 25. März 2019 
Möckel S (2014) Steuerung der Düngung durch Agrar- und Umweltrecht: status quo und rechtspolitische Perspektiven. Vortrag im Rahmen der Münsteraner Gespräche zum Umwelt- und Planungsrecht am 07. Mai 2014. Münster

Möckel S, Reese M (2010) Integrierte Umweltbeobachtung als Grundlage eines effektiven KlimaAnpassungsmanagements. In: Reese M, Möckel S, Bovet J, Köck W (Helmholtz-Institut für Umweltforschung) Rechtlicher Handlungsbedarf für die Anpassung an die Folgen des Klimawandels - Analyse, Weiter- und Neuentwicklung rechtlicher Instrumente. Herausgegeben vom Umweltbundesamt. Schmidt, Dessau-Roßlau, S 403-408

Möhring B, Mestemacher U (2009) Gesellschaftliche Leistungen der Wälder und der Forstwirtschaft und ihre Honorierung. vTI Landbauforschung Sonderheft 327. Waldstrategie 2020 Tagungsband zum Symposium des BMELV am 10. und 11. Dezember 2008, Berlin, S 65-73

Morgan DL (1996) Focus groups as qualitative research (Qualitative Research Methods Series 16), 2. Aufl. Sage, Thousand Oaks

Mueller M, Job H (2009) Managing natural disturbance in protected areas: tourists' attitude towards the bark beetle in a German national park. Biol Cons 142(2):375-383

Mutz D, Matzinger A, Remy C (2013) Maßnahmen zur Reduktion der Nährstoffeinträge urbaner Bereiche. NITROLIMIT - Diskussionspapier, Bd 2, September 2013. Bad Saarow

Neubert L (2014) Integration von Klimaschutz und Anpassung an den Klimawandel in die ländliche Regionalentwicklung - mit informellen Instrumenten ans Ziel? In: Grotheer S, Schwöbel A, Stepper M (Hrsg) Nimm's sportlich - Planung als Hindernislauf. Arbeitsberichte der ARL 10. Hannover, S 159-171

Núñez D, Nahuelhual L, Oyarzún C (2006) Forests and water: the value of native temperate forests in supplying water for human consumption. Ecol Econ 58(3):606-616

Oates WE, Portney PR (2003) The political economy of environmental policy, handbook of environmental economics. Elsevier, Amsterdam, S 325-354

Olschewski R et al (2012) Avalanche protection by forests - a choice-experiment in the Swiss Alps. For Policy Econ 15:108-113

Online Etymology Dictionary (2015) Stichworte „stake“ und „stake-holder“. https://www. etymonline.com/. Zugegriffen: 4. Sept. 2015

Oppermann R, Kasperczyk N, Matzdorf B, Reutter M, Meyer C, Luick R, Stein S, Ameskamp K, Gelhausen J, Bleil R (2013) Reform der Gemeinsamen Agrarpolitik (GAP) 2013 und Erreichung der Biodiversitäts- und Umweltziele. Naturschutz und Biologische Vielfalt 135. Herausgegeben vom Bundesamt für Naturschutz (BfN). Selbstverlag, Bonn

Radtke L, Schliep R, Heiland S (2015) Berücksichtigung des Klimawandels in der Landschaftsrahmenplanung. Ein bundesweiter Überblick. Naturschutz und Landschaftsplanung 47(7):201208

Rajmis S, Barkmann J, Marggraf R (2009) User community preferences for climate change mitigation and adaptation measures around Hainich National Park, Germany. Clim Res 40:61-73

Reed MS, Graves A, Dandy N, Posthumus H, Hubacek K, Morris J, Prell C, Quinn CH, Stringer LC (2009) Who's in and why? A typology of stakeholder analysis methods for natural resource management. J Environ Manage 90(5):1933-1949

Reese M (2011) Das neue Recht des Hochwasserschutzes vor den Herausforderungen des Klimawandels. NuR 33:19-28

Reese M, Köck W, Möckel S (2010) Räumliche Gesamtplanung. In: Reese M, Möckel S, Bovet J, Köck W (Helmholtz-Institut für Umweltforschung) Rechtlicher Handlungsbedarf für die Anpassung an die Folgen des Klimawandels - Analyse, Weiter- und Neuentwicklung rechtlicher Instrumente. Herausgegeben vom Umweltbundesamt. Schmidt, Dessau-Roßlau, S 336-402 
Renn O (2008) Anforderungen an eine integrative und transdisziplinäre Umweltforschung. In: Bergmann M, Schramm E (Hrsg) Transdisziplinäre Forschung - Integrative Forschungsprozesse verstehen und bewerten. Campus, Frankfurt a. M., S 119-148

Ring I, Wüstemann H, Bonn A, Grunewald K, Hampicke U, Hartje V, Jax K, Marzelli S, Meyerhoff J, Schweppe-Kraft B (2015) Methodische Grundlagen zu Ökosystemleistungen und ökonomischer Bewertung. In: Hartje V, Wüstemann H, Bonn A (Hrsg) Naturkapital und Klimapolitik: Synergien und Konflikte. Naturkapital Deutschland - TEEB DE. TUB \& UFZ, Berlin \& Leipzig, S 20-64

Röder N, Osterburg B, Liebersbach H, Bormann K (2014) Faktencheck Agrarreform. Beitrag der EU-Agrarreform zur Bewirtschaftung organischer Böden im Einklang mit Natur und Klimaschutz. Thünen Working Paper 24. Braunschweig

Roovers P, Hermy M, Gulinck H (2002) Visitor profile, perceptions and expectations in forests from a gradient of increasing urbanisation in central Belgium. Landscape Urban Plann 59(3):129-145

Rösch C, Jörissen J, Skarka J, Hartlieb N (2008) Flächennutzungskonflikte: Ursachen Folgen und Lösungsansätze. Technikfolgeabschätzungen - Theorie und Praxis 17(2):4-11

Rüter S, Rock J, Köthke M, Dieter M (2011) Wieviel Holznutzung ist gut fürs Klima? $\mathrm{CO}_{2}$ Bilanzen unterschiedlicher Nutzungsszenarien 2013 bis 2020. AFZ - DerWald 15:19-21

Ryffel AN et al (2014) Land use trade-offs for flood protection: a choice-experiment with visualizations. Ecosyst Serv 10:111-123

Sachverständigenrat für Umweltfragen (SRU) (2012) Umweltgutachten 2012. Verantwortung in einer begrenzten Welt. Schmidt, Berlin

Sachverständigenrat für Umweltfragen (SRU) (2015) Stickstoff: Lösungsstrategien für ein drängendes Umweltproblem. Sondergutachten, Hausdruck

Schroeder CH (2010) Public choice and environmental policy: a review of the literature. In: Farber DA, O'Connell AJ (Hrsg) Research handbook on public choice and public law, Edward Elgar Publishing Ltd., Cheltenham S 450-485

Schuler J, Bues A, Henseler M, Krämer C, Krampe L, Kreins P, Liebersbach H, Osterburg B, Röder N, Uckert G (2014) Instrumente zur Stärkung von Synergien zwischen Naturund Klimaschutz im Bereich Landbewirtschaftung. Ergebnisse des F+E-Vorhabens FKZ 3511880200. BfN-Skripten 382, Bonn

Schwermer S, Preiss P, Müller W (2012) Best-Practice-Kostensätze für Luftschadstoffe, Verkehr, Strom- und Wärmeerzeugung, Anhang B der „Methodenkonvention 2.0 zur Schätzung von Umweltkosten“. Dessau

Seifert P (2012) Mit Sicherheit wächst der Schaden. Überlegungen zum Umgang mit Hochwasser in der räumlichen Planung. Herausgegeben von der Geschäftsstelle des regionalen Planungsverbandes Oberes Elbtal/Osterzgebirge. https://www.klimamoro.de/fileadmin/Dateien/ Ver\%C3\%B6ffentlichungen/Ver\%C3\%B6ffentlichungen_Phase_II/mit_sicherheit_waechst_ der_schaden.pdf. Zugegriffen: 7. Juli 2015

Siewert S (2010) Die Ermittlung kosteneffektiver Maßnahmen zur Reduktion von Nährstoffemissionen aus Kläranlagen im Einzugsgebiet der Oder. Schriftenreihe des IÖW 197/10, Berlin

Spiekermann J, Franck E (Hrsg) (2014) Anpassung an den Klimawandel in der räumlichen Planung - Handlungsempfehlungen für die niedersächsische Planungspraxis auf Landes- und Regionalebene. Arbeitsberichte der ARL 11. Hannover

Springer O (Hrsg) (1963) Langenscheidts Enzyklopädisches Wörterbuch der englischen und deutschen Sprache. Langenscheidt, Berlin

Ssymank A, Scherfose V (2012) Sicherung von Mooren durch Schutzgebiete am Beispiel von Natura 2000 und Großschutzgebieten. Natur und Landschaft 2:62-69 
Steinführer A, Kuhlicke C, De Marchi B, Scolobig A, Tapsell S, Tunstall S (2009) Local communities at risk from flooding: social vulnerability resilience and recommendations for flood risk management in Europe. Helmholtz Centre for Environmental Research - UFZ, Leipzig

Steinhäußer R, Siebert R, Steinführer A, Hellmich M (2015) National and regional land-use conflicts in Germany from the perspective of stakeholders. Land Use Policy 49:183-194

Umweltbundesamt (UBA) (2012) Ökonomische Bewertung von Umweltschäden - Methodenkonvention 2.0 zur Schätzung von Umweltkosten, Dessau: Umweltbundesamt. S 74, Anhang B. S 44

Umweltbundesamt (2019) Methodenkonvention 3.0 zur Ermittlung von Umweltkosten. DessauRoßlau

Unmüssig B (2015) Vom Wert der Natur. Ökologisches Wirtschaften 30:11

van Zanten BT, Verburg PH, Koetse MJ, van Beukering PJ (2014) Preferences for European agrarian landscapes: A meta-analysis of case studies. Landscape and Urban Planning 132:89-101

Verband Region Stuttgart (2009) Region Stuttgart. Regionalplan. Satzungsbeschluss vom 22. Juli 2009. Stuttgart

von der Dunk A, Grêt-Regamey A, Dalanga T, Hersperger AM (2011) Defining a typology of periurban land-use conflicts - a case study from Switzerland. Landscape Urban Plann 101(2):149-156

Voss M (2010) Einleitung: Perspektiven sozialwissenschaftlicher Klimawandelforschung. In: Voss M (Hrsg) Der Klimawandel. Sozialwissenschaftliche Perspektiven. VS Verlag, Wiesbaden, S 9-40

Walter J (2013) Kommunaler Klimaschutz und Mobilität: Da bewegt sich was. In: Service- und Kompetenzzentrum: Kommunaler Klimaschutz beim Deutschen Institut für Urbanistik (Hrsg) Klimaschutz \& Mobilität. Beispiele aus der kommunalen Praxis und Forschung - so lässt sich was bewegen. Deutsches Institut für Urbanistik Köln, S 6-15

Wehn U, Rusca M, Evers J, Lanfranchi V (2015) Participation in flood risk management and the potential of citizen observatories: a governance analysis. Environ Sci Policy 48:225-236

Weingarten P (2014) Ländliche Entwicklung - Anforderungen und Chancen aus Sicht der Wissenschaft. Landentwicklung aktuell 2014, S 48-51

Wichtmann W, Haberl A (2012) Aktivierung der Klimaschutzfunktion von Niedermoorflächen in der Landeshauptstadt Potsdam. Handlungsleitfaden „Paludikultur“. Michael Succow Stiftung zum Schutz der Natur. Auftraggeber Stadtverwaltung Potsdam, Potsdam

Wilke C, Bachmann J, Hage G, Heiland S (Hrsg) (2011) Planungs- und Managementstrategien des Naturschutzes im Lichte des Klimawandels. Naturschutz und Biologische Vielfalt 109. Herausgegeben vom Bundesamt für Naturschutz (BfN). Selbstverlag, Bonn

Wille V, Preiss P, Friedrich R (2012) Sachstandspapier zu Treibhausgase \& Klimawandel, Sachstandspapier im Rahmen des Vorhabens „Schätzung Externer Umweltkosten und Vorschläge zur Kosteninternalisierung in ausgewählten Politikfeldern“, Umweltbundesamt, Forschungsprojekte FKZ 370814 101. Universität Stuttgart, Institut für Energiewirtschaft und Rationelle Energieanwendung

Winkel G (2015) Anpassungen in der Forstwirtschaft undderenAuswirkungen. Infobox 6.1. In: Naturkapital Deutschland - TEEB DE (2015) Naturkapital und Klimapolitik - Synergien und Konflikte (Hrsg) von Volkmar Hartje, Henry Wüstemann und Aletta Bonn. Technische Universität Berlin, Helmholtz-Zentrum für Umweltforschung - UFZ, Berlin, Leipzig, S 154

Wissenschaftlicher Beirat für Agrarpolitik (WBA) (2010) EU-Agrarpolitik nach 2013. Plädoyer für eine neue Politik für Ernährung, Landwirtschaft und ländliche Räume. Gutachten. Bonn, Berlin. www.bmelv.de/beirat_agrarpolitik_gutachten. Zugegriffen: 12. März 2018 
Wolfram M, Albrecht J, Wulfhorst G, Horn B, Krebser S, Verron H, Holz-Rau C (2010) Steuerung einer nachhaltigen Verkehrsentwicklungsplanung in Deutschland. IÖR Texte 162. Dresden

Yohe GW et al (2007) Perspectives on climate change and sustainability. In: Parry ML, Canziani OF, Palutikof JP, van der Linden PJ, Hanson CE (Hrsg) Climate change 2007: impacts, adaptation and vulnerability. Contribution of working group II to the fourth assessment report of the intergovernmental panel on climate change. Cambridge University Press, Cambridge, $S$ $811-841$

Zierhofer W, Burger P (2007) Transdisziplinäre Forschung - ein eigenständiger Modus der Wissensproduktion? Problemorientierung, Wissensintegration und Partizipation in transdisziplinären Forschungsprojekten. GAIA 16(1):29-34

Open Access Dieses Kapitel wird unter der Creative Commons Namensnennung 4.0 International Lizenz (http://creativecommons.org/licenses/by/4.0/deed.de) veröffentlicht, welche die Nutzung, Vervielfältigung, Bearbeitung, Verbreitung und Wiedergabe in jeglichem Medium und Format erlaubt, sofern Sie den/die ursprünglichen Autor(en) und die Quelle ordnungsgemäß nennen, einen Link zur Creative Commons Lizenz beifügen und angeben, ob Änderungen vorgenommen wurden.

Die in diesem Kapitel enthaltenen Bilder und sonstiges Drittmaterial unterliegen ebenfalls der genannten Creative Commons Lizenz, sofern sich aus der Abbildungslegende nichts anderes ergibt. Sofern das betreffende Material nicht unter der genannten Creative Commons Lizenz steht und die betreffende Handlung nicht nach gesetzlichen Vorschriften erlaubt ist, ist für die oben aufgeführten Weiterverwendungen des Materials die Einwilligung des jeweiligen Rechteinhabers einzuholen.

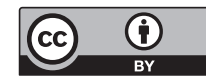

